Supplementary Information

\title{
Bio-Patterned Reorganization of Alkaloids Enabled by Ring-Opening Functionalization of Tertiary Amines
}

Hyeonggeun Lim, ${ }^{\dagger, \S}$ Sikwang Seong, ${ }^{\dagger, \S}$ Youyoung Kim, ${ }^{\dagger, \neq}$ Sangwon Seo, ${ }^{\dagger, \ddagger}$ and Sunkyu Han ${ }^{\dagger, \ddagger, *}$

†Department of Chemistry, Korea Advanced Institute of Science \& Technology (KAIST), Daejeon 34141, Korea

*Center for Catalytic Hydrocarbon Functionalizations, Institute for Basic Science (IBS), Daejeon 34141, Korea

§These authors contributed equally to this work.

*Corresponding author

Table of Contents

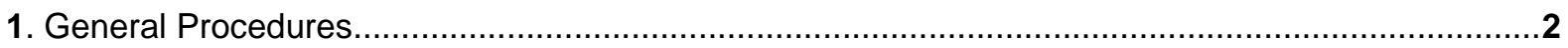

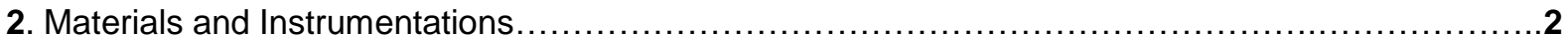

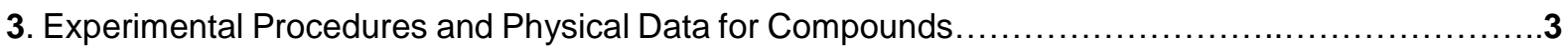

4. Comparison of Spectroscopic Data of Natural and Synthetic Compounds..........................50

5. X-ray Crystal Structure Determination of Nominal (+)-Tabercarpamine G (27) ......................66

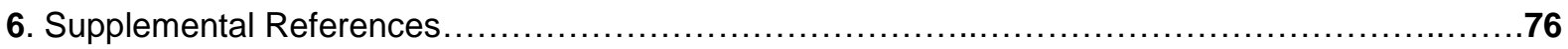

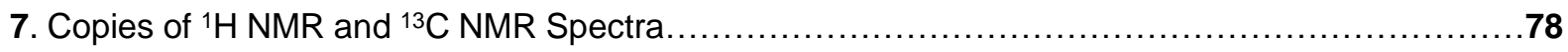




\section{General Procedures}

All reactions were performed in oven-dried flasks. Unless otherwise noted, the flasks were fitted with rubber septa and reactions were conducted under a positive pressure of argon. Stainless steel syringes or cannula were used to transfer air- and moisture-sensitive liquids. Flash column chromatography was performed as described by Still et al. using silica gel (60-Å pore size, $40-63 \mu \mathrm{m}$, $4-6 \% \mathrm{H}_{2} \mathrm{O}$ content, Merck). ${ }^{1}$ Analytical thin-layer chromatography (TLC) was performed using glass plates pre-coated with $0.25 \mathrm{~mm}$ silica gel impregnated with a fluorescent indicator $(254 \mathrm{~nm})$. Thin layer chromatography plates were visualized by exposure to ultraviolet light, an aqueous solution of ceric ammonium molybdate (CAM) and/or a potassium permanganate $\left(\mathrm{KMnO}_{4}\right)$.

\section{Materials and Instrumentations}

Unless otherwise stated, all commercial reagents and solvents were used without additional purification with the following exceptions: dichloromethane and tetrahydrofuran were purchased from Merck and Daejung Inc., respectively and were purified by the method of Grubbs et al. under positive argon pressure. ${ }^{2}(+)$-Catharanthine (3) was purchased from JHChem $(50 \mathrm{~g} / \$ 1,350)$. Commercially available $3(2.0 \mathrm{~g})$ was diluted in ethyl acetate $(10 \mathrm{~mL})$ and saturated aqueous sodium bicarbonate solution $(10 \mathrm{~mL})$ and the layers were separated. The organic layers were dried over anhydrous sodium sulfate and concentrated under reduced pressure. This resulting (+)-catharanthine was used without further purification. Allosecurinine was purchased from $\mathrm{J} \& \mathrm{H}(10 \mathrm{~g} / \$ 6,230)$.

Proton and carbon nuclear magnetic resonance spectra were recorded with Bruker Avance 400 (400 MHz), Bruker Avance III HD (400 MHz), Bruker Avance III HD Nano bay (400 MHz), Bruker Avance Neo (500 MHz), Agilent Technologies DD2 (600 MHz). Proton nuclear magnetic resonance spectra are referenced from the residual protium in the NMR solvent $\left(\mathrm{CDCl}_{3}: \delta 7.24, \mathrm{CD}_{3} \mathrm{OD}: \delta .31\right)$. Data are reported in the following manners: chemical shift in ppm [multiplicity ( $s=$ singlet, $d=$ doublet, $\mathrm{t}=$ triplet, $\mathrm{q}=$ quartet, $\mathrm{p}=$ quintet, $\mathrm{m}=$ multiplet, $\mathrm{app}=$ apparent, $\mathrm{br}=$ broad), coupling constant(s) in Hertz, integration]. Carbon-13 nuclear magnetic resonance spectra are referenced from the carbon resonances of the solvent $\left(\mathrm{CDCl}_{3}: \delta 77.23, \mathrm{CD}_{3} \mathrm{OD}: \delta 49.00\right)$. Data are reported in the following manners: chemical shift in ppm. High resolution mass spectra were obtained from KAIST Research Analysis Center (Daejeon) by using an ESI ionization method. Specific rotation [a]D was obtained by JASCO P2000 polarimeter. 


\section{Experimental Procedures and Physical Data for Compounds}<smiles>c1ccc(N2CCCCC2)cc1</smiles>

10

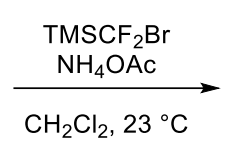

$51 \%$<smiles>FC(F)(F)[N+]1(c2ccccc2Br)CCCCC1</smiles>

11

\section{1-(difluoromethyl)-1-phenylpiperidin-1-ium Bromide (11):}

To a solution of 10 (80.6 mg, $0.5 \mathrm{mmol}, 1$ equiv) in $\mathrm{CH}_{2} \mathrm{Cl}_{2}\left(2 \mathrm{~mL}\right.$ ) was added $\mathrm{NH}_{4} \mathrm{OAc}$ (154.16 $\mathrm{mg}, 2 \mathrm{mmol}, 4$ equiv) and $\mathrm{TMSCF}_{2} \mathrm{Br}(0.312 \mathrm{~mL}, 2 \mathrm{mmol}, 4$ equiv). After 12 hours, the crude product was filtered through a pad of celite, washed with $\mathrm{DCM}(10 \mathrm{~mL} \times 3)$ and concentrated under reduced pressure. The residue was then diluted in EtOAc, decanted, and filtered to give 11 (74 mg, 51\%) as a white solid.

${ }^{1} \mathrm{H}$ NMR $\left(400 \mathrm{MHz}, \mathrm{CDCl}_{3}\right): \delta 8.60(\mathrm{t}, \mathrm{J}=59.4 \mathrm{~Hz}, 1 \mathrm{H}), 7.77-7.60(\mathrm{~m}, 5 \mathrm{H}), 5.13(\mathrm{td}, \mathrm{J}=13.2,2.3 \mathrm{~Hz}$, $2 \mathrm{H}), 4.58(\mathrm{~d}, \mathrm{~J}=12.3 \mathrm{~Hz}, 2 \mathrm{H}), 2.07(\mathrm{~d}, \mathrm{~J}=15.2 \mathrm{~Hz}, 2 \mathrm{H}), 1.96(\mathrm{ddd}, \mathrm{J}=14.2,11.0,4.3 \mathrm{~Hz}, 1 \mathrm{H}), 1.77-$ $1.67(\mathrm{~m}, 3 \mathrm{H})$.

${ }^{13} \mathrm{C}$ NMR $\left(101 \mathrm{MHz}, \mathrm{CDCl}_{3}\right): \delta 131.9,131.8,131.2,124.9,114.5(\mathrm{t}, \mathrm{J}=280.1 \mathrm{~Hz}), 56.8,21.0,20.0$.

${ }^{19} \mathrm{~F}$ NMR $\left(376 \mathrm{MHz}, \mathrm{CDCl}_{3}\right): \delta-111.3$.

HRMS (ESI): Calculated for $\mathrm{C}_{12} \mathrm{H}_{16} \mathrm{~F}_{2} \mathrm{~N}^{+}[\mathrm{M}]^{+}: 212.1245$, found: 212.1241.

TLC (dichloromethane : methanol = $9: 1$ ) Rf: 0.1 
<smiles>FC(F)(F)[N+]1(c2ccccc2Br)CCCCC1</smiles>

11

\section{DCE, $60^{\circ} \mathrm{C}$}

$93 \%$<smiles>O=CN(CCCCBr)c1ccccc1</smiles>

13

\section{N-(5-bromopentyl)-N-phenylformamide (13):}

To a solution of 11 (14.6 mg, $0.05 \mathrm{mmol}, 1$ equiv) in DCE $(1.5 \mathrm{~mL})$ and the temperature was raised to $60^{\circ} \mathrm{C}$. After 12 hours, the resulting crude residue was dissolved in ethyl acetate $(5 \mathrm{~mL})$ and saturated aqueous sodium bicarbonate solution $(5 \mathrm{~mL})$ and the layers were separated. The aqueous layer was extracted with ethyl acetate $(3 \times 5 \mathrm{~mL})$, and the combined organic layers were dried over anhydrous sodium sulfate and concentrated under reduced pressure. The resulting crude residue was purified by column chromatography (silica gel: diam. $2 \mathrm{~cm}$, ht. $9 \mathrm{~cm}$; eluent : hexane : ethyl acetate = $1: 1)$ to afford $13(12.5 \mathrm{mg}, 93 \%)$ as a yellow oil.

${ }^{1} \mathrm{H}$ NMR $\left(500 \mathrm{MHz}, \mathrm{CDCl}_{3}\right): \delta 8.39(\mathrm{~s}, 1 \mathrm{H}), 7.44(\mathrm{t}, \mathrm{J}=7.8 \mathrm{~Hz}, 2 \mathrm{H}), 7.33(\mathrm{t}, \mathrm{J}=7.4 \mathrm{~Hz}, 1 \mathrm{H}), 7.21-7.18$ $(\mathrm{m}, 2 \mathrm{H}), 3.87-3.83(\mathrm{~m}, 2 \mathrm{H}), 3.38(\mathrm{t}, \mathrm{J}=6.7 \mathrm{~Hz}, 2 \mathrm{H}), 1.87(\mathrm{dt}, \mathrm{J}=14.3,6.8 \mathrm{~Hz}, 2 \mathrm{H}), 1.59(\mathrm{p}, \mathrm{J}=7.5$ $\mathrm{Hz}, 2 \mathrm{H}), 1.51-1.43(\mathrm{~m}, 2 \mathrm{H})$.

${ }^{13} \mathrm{C}$ NMR (151 MHz, $\left.\mathrm{CDCl}_{3}\right): \delta$ 162.4, 140.8, 129.7, 127.0, 124.3, 44.6, 33.5, 32.2, 26.7, 25.2.

HRMS (ESI): Calculated for $\mathrm{C}_{12} \mathrm{H}_{16} \mathrm{BrNO}[\mathrm{M}+\mathrm{Na}]^{+}: 292.0307$, found: 292.0300 .

TLC (hexane : ethyl acetate $=1: 1)$ Rf: 0.5 (UV). 

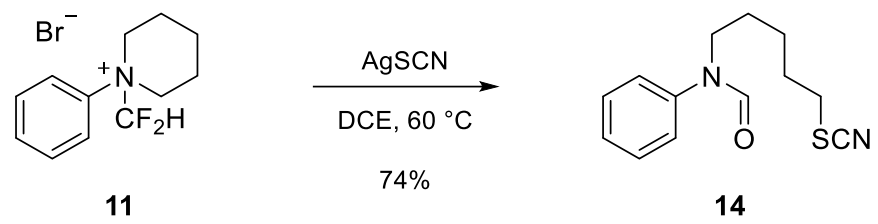

\section{N-phenyl-N-(5-thiocyanatopentyl)formamide (14):}

To a solution of 11 (14.6 mg, $0.05 \mathrm{mmol}, 1$ equiv) in DCE (1.5 mL) was added AgSCN (16.6 $\mathrm{mg}, 0.1 \mathrm{mmol}, 2$ equiv) and stirred at room temperature for $30 \mathrm{~min}$. The temperature was raised to $60^{\circ} \mathrm{C}$. After 12 hours, the resulting crude residue was dissolved in ethyl acetate $(5 \mathrm{~mL})$ and saturated aqueous sodium bicarbonate solution $(5 \mathrm{~mL})$ and the layers were separated. The aqueous layer was extracted with ethyl acetate $(3 \times 5 \mathrm{~mL})$, and the combined organic layers were dried over anhydrous sodium sulfate and concentrated under reduced pressure. The resulting crude residue was purified by column chromatography (silica gel: diam. $2 \mathrm{~cm}$, ht. $9 \mathrm{~cm}$; eluent : hexane : ethyl acetate $=1: 1$ ) to afford $14(9.2 \mathrm{mg}, 74 \%)$ as a yellow oil.

${ }^{1}$ H NMR $\left(400 \mathrm{MHz}, \mathrm{CDCl}_{3}\right)$ : $\delta 8.35(\mathrm{~s}, 1 \mathrm{H}), 7.46-7.38(\mathrm{~m}, 2 \mathrm{H}), 7.29(\mathrm{t}, \mathrm{J}=7.4 \mathrm{~Hz}, 1 \mathrm{H}), 7.16-7.13$ $(\mathrm{m}, 2 \mathrm{H}), 3.82(\mathrm{t}, \mathrm{J}=7.2 \mathrm{~Hz}, 2 \mathrm{H}), 2.88(\mathrm{t}, \mathrm{J}=7.2 \mathrm{~Hz}, 2 \mathrm{H}), 1.80(\mathrm{p}, \mathrm{J}=7.3 \mathrm{~Hz}, 2 \mathrm{H}), 1.57(\mathrm{dt}, \mathrm{J}=14.6$, $7.6 \mathrm{~Hz}, 2 \mathrm{H}), 1.49-1.40(\mathrm{~m}, 2 \mathrm{H})$.

${ }^{13} \mathrm{C}$ NMR $\left(101 \mathrm{MHz}, \mathrm{CDCl}_{3}\right): \delta 162.6,140.9,130.0,127.3,124.4,112.4,44.6,34.0,29.6,27.1,25.2$.

HRMS (ESI): Calculated for $\mathrm{C}_{13} \mathrm{H}_{16} \mathrm{~N}_{2} \mathrm{OS}[\mathrm{M}+\mathrm{Na}]^{+}: 271.0876$, found: 271.0876 .

TLC (hexane : ethyl acetate $=1: 1$ ) Rf: 0.8 (UV). 
<smiles>FC(F)(F)N1CCCCC1</smiles>

11

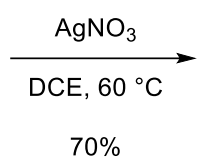

$70 \%$<smiles>O=CN(CCCCC[N+](=O)[O-])c1ccccc1</smiles>

15

\section{5-( $N$-phenylformamido)pentyl nitrate (15):}

To a solution of 11 (14.6 mg, $0.05 \mathrm{mmol}, 1$ equiv) in DCE (1.5 mL) was added $\mathrm{AgNO}_{3}(17 \mathrm{mg}$ $\mathrm{mg}, 0.1 \mathrm{mmol}, 2$ equiv) and stirred at room temperature for $30 \mathrm{~min}$. The temperature was raised to $60^{\circ} \mathrm{C}$. After 12 hours, the resulting crude residue was dissolved in ethyl acetate $(5 \mathrm{~mL})$ and saturated aqueous sodium bicarbonate solution $(5 \mathrm{~mL})$ and the layers were separated. The aqueous layer was extracted with ethyl acetate $(3 \times 5 \mathrm{~mL})$, and the combined organic layers were dried over anhydrous sodium sulfate and concentrated under reduced pressure. The resulting crude residue was purified by column chromatography (silica gel: diam. $2 \mathrm{~cm}$, ht. $9 \mathrm{~cm}$; eluent : hexane : ethyl acetate $=1: 1$ ) to afford $15(8.8 \mathrm{mg}, 70 \%)$ as a yellow oil.

${ }^{1} \mathrm{H}$ NMR $\left(400 \mathrm{MHz}, \mathrm{CDCl}_{3}\right): \delta 8.35(\mathrm{~s}, 1 \mathrm{H}), 7.45-7.36(\mathrm{~m}, 2 \mathrm{H}), 7.29(\mathrm{t}, \mathrm{J}=7.4 \mathrm{~Hz}, 1 \mathrm{H}), 7.18-7.10$ $(\mathrm{m}, 2 \mathrm{H}), 4.38(\mathrm{t}, \mathrm{J}=6.5 \mathrm{~Hz}, 2 \mathrm{H}), 3.88-3.76(\mathrm{~m}, 2 \mathrm{H}), 1.77-1.63(\mathrm{~m}, 2 \mathrm{H}), 1.58(\mathrm{dd}, \mathrm{J}=15.0,7.2 \mathrm{~Hz}$, $2 \mathrm{H}), 1.40(\mathrm{q}, \mathrm{J}=8.7,8.1 \mathrm{~Hz}, 2 \mathrm{H})$.

${ }^{13} \mathrm{C}$ NMR (101 MHz, $\left.\mathrm{CDCl}_{3}\right): \delta 162.6,141.0,130.0,127.2,124.4,73.2,44.6,27.3,26.6,23.1$.

HRMS (ESI): Calculated for $\mathrm{C}_{12} \mathrm{H}_{16} \mathrm{~N}_{2} \mathrm{O}_{4}[\mathrm{M}+\mathrm{Na}]^{+}:$275.1002, found: 275.1003.

TLC (hexane : ethyl acetate $=1: 1$ ) Rf: 0.5 (UV). 
<smiles>FC(F)(F)N1CCCCC1</smiles>

11

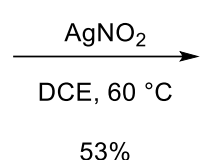

$53 \%$<smiles>O=CN(CCCCC[N+](=O)[O-])c1ccccc1</smiles>

16

\section{$N$-(5-nitropentyl)- $N$-phenylformamide (16):}

To a solution of 11 (14.6 mg, $0.05 \mathrm{mmol}, 1$ equiv) in DCE (1.5 mL) was added $\mathrm{AgNO}_{2}$ (15.3 $\mathrm{mg}, 0.1 \mathrm{mmol}, 2$ equiv) and stirred at room temperature for $30 \mathrm{~min}$. The temperature was raised to $60^{\circ} \mathrm{C}$. After 12 hours, the resulting crude residue was dissolved in ethyl acetate $(5 \mathrm{~mL})$ and saturated aqueous sodium bicarbonate solution $(5 \mathrm{~mL})$ and the layers were separated. The aqueous layer was extracted with ethyl acetate $(3 \times 5 \mathrm{~mL})$, and the combined organic layers were dried over anhydrous sodium sulfate and concentrated under reduced pressure. The resulting crude residue was purified by column chromatography (silica gel: diam. $2 \mathrm{~cm}$, ht. $9 \mathrm{~cm}$; eluent : hexane : ethyl acetate $=1: 1$ ) to afford $16(6.3 \mathrm{mg}, 53 \%)$ as a yellow oil.

${ }^{1} \mathrm{H}$ NMR $\left(400 \mathrm{MHz}, \mathrm{CDCl}_{3}\right): \delta 8.35(\mathrm{~s}, 1 \mathrm{H}), 7.45-7.38(\mathrm{~m}, 2 \mathrm{H}), 7.29(\mathrm{t}, \mathrm{J}=7.4 \mathrm{~Hz}, 1 \mathrm{H}), 7.17-7.12$ $(\mathrm{m}, 2 \mathrm{H}), 4.38(\mathrm{t}, \mathrm{J}=6.6 \mathrm{~Hz}, 2 \mathrm{H}), 3.86-3.78(\mathrm{~m}, 2 \mathrm{H}), 1.75-1.64(\mathrm{~m}, 2 \mathrm{H}), 1.57(\mathrm{dt}, \mathrm{J}=14.9,7.4 \mathrm{~Hz}$, $2 \mathrm{H}), 1.40(\mathrm{q}, \mathrm{J}=8.7,8.1 \mathrm{~Hz}, 2 \mathrm{H})$.

${ }^{13} \mathrm{C}$ NMR (101 MHz, $\left.\mathrm{CDCl}_{3}\right): \delta$ 162.6, 141.0, 130.0, 127.3, 124.4, 73.2, 44.7, 27.3, 26.6, 23.1.

HRMS (ESI): Calculated for $\mathrm{C}_{12} \mathrm{H}_{16} \mathrm{~N}_{2} \mathrm{O}_{3}[\mathrm{M}+\mathrm{Na}]^{+}:$259.1053, found: 259.1024 .

TLC (hexane : ethyl acetate $=1: 1) \mathrm{R} f: 0.4$ (UV). 
<smiles>FC(F)(F)[N+]1(c2ccccc2)CCCCC1</smiles>

11

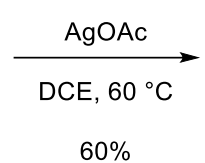

$60 \%$<smiles>CC(=O)OCCCCCN(C=O)c1ccccc1</smiles>

17

\section{5-(N-phenylformamido)pentyl acetate (17):}

To a solution of 11 (14.6 mg, $0.05 \mathrm{mmol}, 1$ equiv) in DCE (1.5 mL) was added AgOAc (16.7 $\mathrm{mg}, 0.1 \mathrm{mmol}, 2$ equiv) and stirred at room temperature for $30 \mathrm{~min}$. The temperature was raised to $60^{\circ} \mathrm{C}$. After 12 hours, the resulting crude residue was dissolved in ethyl acetate $(5 \mathrm{~mL})$ and saturated aqueous sodium bicarbonate solution $(5 \mathrm{~mL}$ ) and the layers were separated. The aqueous layer was extracted with ethyl acetate $(3 \times 5 \mathrm{~mL})$, and the combined organic layers were dried over anhydrous sodium sulfate and concentrated under reduced pressure. The resulting crude residue was purified by column chromatography (silica gel: diam. $2 \mathrm{~cm}$, ht. $9 \mathrm{~cm}$; eluent : hexane : ethyl acetate $=1: 1$ ) to afford 17 (7.5 mg, 3 steps $60 \%)$ as a yellow oil.

${ }^{1} \mathrm{H}$ NMR $\left(400 \mathrm{MHz}, \mathrm{CDCl}_{3}\right)$ : $\delta 8.35(\mathrm{~s}, 1 \mathrm{H}), 7.43-7.36(\mathrm{~m}, 2 \mathrm{H}), 7.28(\mathrm{t}, \mathrm{J}=7.4 \mathrm{~Hz}, 1 \mathrm{H}), 7.14(\mathrm{dd}, \mathrm{J}=$ 8.5, $1.2 \mathrm{~Hz}, 2 \mathrm{H}), 3.99(\mathrm{t}, \mathrm{J}=6.6 \mathrm{~Hz}, 2 \mathrm{H}), 3.85-3.76(\mathrm{~m}, 2 \mathrm{H}), 2.00(\mathrm{~s}, 3 \mathrm{H}), 1.63-1.47(\mathrm{~m}, 4 \mathrm{H}), 1.40-$ $1.28(\mathrm{~m}, 2 \mathrm{H})$.

${ }^{13} \mathrm{C}$ NMR $\left(101 \mathrm{MHz}, \mathrm{CDCl}_{3}\right): \delta 171.4,162.6,141.1,129.9,127.2,124.4,64.4,44.9,28.4,27.5,23.4$, 21.2 .

HRMS (ESI): Calculated for $\mathrm{C}_{14} \mathrm{H}_{19} \mathrm{NO}_{3}[\mathrm{M}+\mathrm{Na}]^{+}:$272.1257, found: 272.1255.

TLC (hexane : ethyl acetate $=1: 1$ ) Rf: 0.5 (UV). 
<smiles>FC(F)(F)[N+]1(c2ccccc2)CCCCC1</smiles>

11

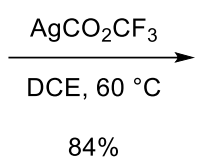

$84 \%$<smiles>O=CN(CCCCO)c1ccccc1</smiles>

18

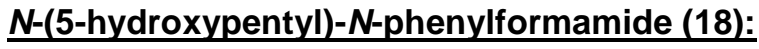

To a solution of 11 (14.6 mg, $0.05 \mathrm{mmol}, 1$ equiv) in DCE (1.5 mL) was added $\mathrm{AgCO}_{2} \mathrm{CF}_{3}$ (22 $\mathrm{mg}, 0.1 \mathrm{mmol}, 2$ equiv) and stirred at room temperature for $30 \mathrm{~min}$. The temperature was raised to $60^{\circ} \mathrm{C}$. After 12 hours, the resulting crude residue was dissolved in ethyl acetate $(5 \mathrm{~mL})$ and saturated aqueous sodium bicarbonate solution $(5 \mathrm{~mL}$ ) and the layers were separated. The aqueous layer was extracted with ethyl acetate $(3 \times 5 \mathrm{~mL})$, and the combined organic layers were dried over anhydrous sodium sulfate and concentrated under reduced pressure. The resulting crude residue was purified by column chromatography (silica gel: diam. $2 \mathrm{~cm}$, ht. $9 \mathrm{~cm}$; eluent : hexane : ethyl acetate $=1: 1$ ) to afford $18(8.7 \mathrm{mg}, 84 \%)$ as a colorless oil.

${ }^{1} \mathrm{H}$ NMR $\left(600 \mathrm{MHz}, \mathrm{CDCl}_{3}\right): \delta 8.35(\mathrm{~s}, 1 \mathrm{H}), 7.40(\mathrm{t}, \mathrm{J}=7.7 \mathrm{~Hz}, 2 \mathrm{H}), 7.29(\mathrm{t}, \mathrm{J}=7.4 \mathrm{~Hz}, 1 \mathrm{H}), 7.14(\mathrm{~d}, \mathrm{~J}$ $=7.8 \mathrm{~Hz}, 2 \mathrm{H}), 4.28(\mathrm{t}, \mathrm{J}=6.6 \mathrm{~Hz}, 2 \mathrm{H}), 3.82(\mathrm{t}, \mathrm{J}=7.4 \mathrm{~Hz}, 2 \mathrm{H}), 1.74-1.68(\mathrm{~m}, 2 \mathrm{H}), 1.61-1.54(\mathrm{~m}$, $2 \mathrm{H}), 1.47-1.35(\mathrm{~m}, 2 \mathrm{H})$.

${ }^{13} \mathrm{C}$ NMR (151 MHz, $\left.\mathrm{CDCl}_{3}\right): \delta$ 162.6, 141.0, 130.0, 127.2, 124.4, 68.1, 44.6, 27.9, 27.2, 23.0.

HRMS (ESI): Calculated for $\mathrm{C}_{12} \mathrm{H}_{17} \mathrm{NO}_{2}[\mathrm{M}+\mathrm{Na}]^{+}: 230.1151$, found: 230.1164 .

TLC (hexane : ethyl acetate $=1: 1$ ) Rf: 0.5 (UV). 
<smiles>FC(F)(F)[N+]1(c2ccccc2Br)CCCCC1</smiles>

11

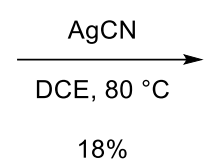

$18 \%$<smiles>N#CCCCCCN(C=O)c1ccccc1</smiles>

19

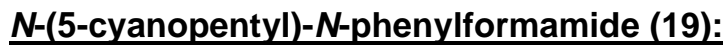

To a solution of 11 (20.9 mg, $0.0715 \mathrm{mmol}, 1$ equiv) in DCE (1mL) was added AgCN (19.2 mg, $0.143 \mathrm{mmol}, 2$ equiv) and stirred at room temperature for $30 \mathrm{~min}$. The temperature was raised to $80^{\circ} \mathrm{C}$. After 12 hours, the resulting crude residue was dissolved in ethyl acetate $(5 \mathrm{~mL})$ and saturated aqueous sodium bicarbonate solution $(5 \mathrm{~mL})$ and the layers were separated. The aqueous layer was extracted with ethyl acetate $(3 \times 5 \mathrm{~mL})$, and the combined organic layers were dried over anhydrous sodium sulfate and concentrated under reduced pressure. The resulting crude residue was purified by column chromatography (silica gel: diam. $2 \mathrm{~cm}$, ht. $9 \mathrm{~cm}$; eluent : hexane : ethyl acetate $=1: 1$ ) to afford 19 $(2.8 \mathrm{mg}, 18 \%)$ as a yellow oil.

${ }^{1} \mathrm{H}$ NMR $\left(400 \mathrm{MHz}, \mathrm{CDCl}_{3}\right): \delta 8.36(\mathrm{~s}, 1 \mathrm{H}), 7.41(\mathrm{dd}, \mathrm{J}=8.4,7.1 \mathrm{~Hz}, 2 \mathrm{H}), 7.30(\mathrm{t}, \mathrm{J}=7.4 \mathrm{~Hz}, 1 \mathrm{H}), 7.15$ (dd, J = 8.5, $1.3 \mathrm{~Hz}, 2 \mathrm{H}), 3.82(\mathrm{t}, \mathrm{J}=7.2 \mathrm{~Hz}, 2 \mathrm{H}), 3.33(\mathrm{ddt}, \mathrm{J}=6.6,3.9,1.9 \mathrm{~Hz}, 2 \mathrm{H}), 1.70-1.63(\mathrm{~m}$, $2 \mathrm{H}), 1.56(\mathrm{q}, \mathrm{J}=7.5 \mathrm{~Hz}, 4 \mathrm{H}), 1.47-1.38(\mathrm{~m}, 2 \mathrm{H})$.

${ }^{13} \mathrm{C}$ NMR (101 MHz, $\left.\mathrm{CDCl}_{3}\right): \delta$ 162.6, 156.0 (HMBC), 141.0, 130.0, 127.3, 124.5, 44.6, 41.5, 28.8, 26.9, 23.7.

HRMS (ESI): Calculated for $\mathrm{C}_{13} \mathrm{H}_{16} \mathrm{~N}_{2} \mathrm{O}[\mathrm{M}+\mathrm{Na}]^{+}: 239.1155$, found: 239.1143.

TLC (hexane : ethyl acetate $=1: 1$ ) Rf: 0.45 (UV). 

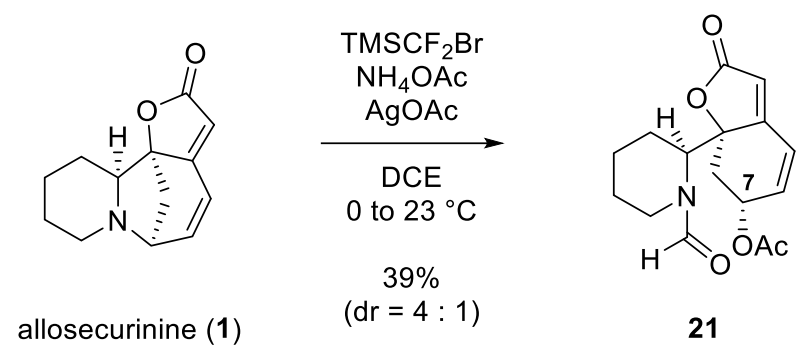

\section{formamide 21:}

To a stirred solution of allosecurinine (1) $(100 \mathrm{mg}, 0.46 \mathrm{mmol}, 1$ equiv) in 1.2-dichloroethane (8 $\mathrm{mL}$ ) was added $\mathrm{NH}_{4} \mathrm{OAc}$ (142 mg, $1.841 \mathrm{mmol}, 4$ equiv) and $\mathrm{AgOAc}$ (307 mg, $1.841 \mathrm{mmol}, 4$ equiv) at $0^{\circ} \mathrm{C}$. After $5 \mathrm{~min}, \mathrm{TMSCF}_{2} \mathrm{Br}(0.14 \mathrm{~mL}, 0.921 \mathrm{mmol}, 2$ equiv) was added dropwise and the temperature was raised to room temperature. After 3 hours, the crude product was filtered through a pad of celite, washed with dichloromethane $(10 \mathrm{~mL})$ and the resulting mixture was diluted with dichloromethane (10 $\mathrm{mL}$ ) and saturated aqueous sodium chloride solution $(10 \mathrm{~mL})$ and the layers were separated. The aqueous layer was extracted with dichloromethane $(3 \times 10 \mathrm{~mL})$, and the combined organic layers were dried over anhydrous sodium sulfate and concentrated under reduced pressure. The resulting crude residue was purified by column chromatography (silica gel: diam. $3 \mathrm{~cm}$, ht. $9 \mathrm{~cm}$; eluent : hexane : ethyl acetate $=2: 1)$ to afford $21(55.1 \mathrm{mg}, 39 \%)$ as a white solid.

${ }^{1} \mathrm{H}$ NMR (major) $\left(500 \mathrm{MHz}, \mathrm{CDCl}_{3}\right): \delta 7.87(\mathrm{~s}, 1 \mathrm{H}), 6.65$ (dd, $\left.\mathrm{J}=10.0,2.2 \mathrm{~Hz}, 1 \mathrm{H}\right), 6.08$ (dt, J = 10.1, $1.8 \mathrm{~Hz}, 1 \mathrm{H}), 5.76(\mathrm{~s}, 1 \mathrm{H}), 5.55$ (ddd, J = 10.6, $5.5,2.6 \mathrm{~Hz}, 1 \mathrm{H}), 4.40$ (dd, J = 7.0, $4.0 \mathrm{~Hz}, 1 \mathrm{H}$ ), $3.40-$ $3.32(\mathrm{~m}, 2 \mathrm{H}), 2.93(\mathrm{dd}, \mathrm{J}=12.3,5.6 \mathrm{~Hz}, 1 \mathrm{H}), 2.17(\mathrm{ddq}, \mathrm{J}=13.8,6.8,3.4 \mathrm{~Hz}, 1 \mathrm{H}), 2.06(\mathrm{~s}, 3 \mathrm{H}), 1.95-$ $1.87(\mathrm{~m}, 1 \mathrm{H}), 1.84-1.79(\mathrm{~m}, 1 \mathrm{H}), 1.75(\mathrm{dt}, \mathrm{J}=11.4,4.0 \mathrm{~Hz}, 2 \mathrm{H}), 1.63-1.55(\mathrm{~m}, 1 \mathrm{H}), 1.47$ (dddd, $\mathrm{J}=$ $13.3,11.8,6.8,3.3 \mathrm{~Hz}, 1 \mathrm{H})$.

${ }^{13} \mathrm{C}$ NMR (major) (151 MHz, $\left.\mathrm{CDCl}_{3}\right): \delta 171.9,170.3,164.9,162.9,135.1,123.7,112.6,88.7,67.4$, 49.9, 44.1, 37.8, 25.1, 24.3, 21.1, 19.7.

${ }^{1} \mathrm{H}$ NMR (minor) $\left(500 \mathrm{MHz}, \mathrm{CDCl}_{3}\right): \delta 7.60(\mathrm{~s}, 1 \mathrm{H}), 6.65-6.61(\mathrm{~m}, 1 \mathrm{H}), 6.17(\mathrm{dt}, \mathrm{J}=10.1,1.8 \mathrm{~Hz}, 1 \mathrm{H})$, $5.90(\mathrm{~s}, 1 \mathrm{H}), 5.51(\mathrm{dt}, \mathrm{J}=8.2,2.4 \mathrm{~Hz}, 1 \mathrm{H}), 4.22(\mathrm{dd}, \mathrm{J}=13.6,5.8 \mathrm{~Hz}, 1 \mathrm{H}), 3.62(\mathrm{dd}, \mathrm{J}=6.9,2.7 \mathrm{~Hz}$, $1 \mathrm{H}), 3.31(\mathrm{~d}, \mathrm{~J}=4.3 \mathrm{~Hz}, 1 \mathrm{H}), 2.77(\mathrm{td}, \mathrm{J}=13.1,4.3 \mathrm{~Hz}, 1 \mathrm{H}), 2.30-2.22(\mathrm{~m}, 1 \mathrm{H}), 2.08(\mathrm{~s}, 3 \mathrm{H}), 1.99(\mathrm{dt}$, $\mathrm{J}=12.2,3.6 \mathrm{~Hz}, 1 \mathrm{H}), 1.88-1.84(\mathrm{~m}, 2 \mathrm{H}), 1.70(\mathrm{~d}, \mathrm{~J}=4.0 \mathrm{~Hz}, 1 \mathrm{H}), 1.65(\mathrm{t}, \mathrm{J}=4.3 \mathrm{~Hz}, 1 \mathrm{H}), 1.40-1.29$ $(\mathrm{m}, 1 \mathrm{H})$.

${ }^{13} \mathrm{C}$ NMR (minor) $\left(151 \mathrm{MHz}, \mathrm{CDCl}_{3}\right): \delta 170.8,170.1,163.0,162.5,137.1,122.1,114.6,89.4,67.0$, $54.7,37.8,37.7,24.9,23.7,20.9,19.8$.

HRMS (ESI): Calculated for $\mathrm{C}_{16} \mathrm{H}_{19} \mathrm{NO}_{5}[\mathrm{M}+\mathrm{Na}]^{+}: 328.1155$, found: 328.1162.

TLC (ethyl acetate) Rf: 0.45 (UV). 
<smiles>CC(=O)O[C@H]1C=CC2=CC(=O)O[C@]2(C2CCCCN2C(=O)O)C1</smiles>

21

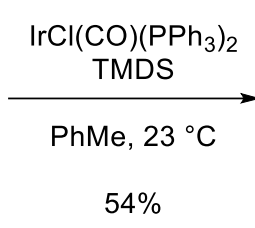

$54 \%$<smiles>CC(=O)O[C@H]1C=CC2=CC(=O)O[C@]2(C2CCCCN2)C1</smiles>

s1

\section{amine S1:}

To a solution of 21 (45.9 mg, $0.1503 \mathrm{mmol}, 1$ equiv) in toluene $(5 \mathrm{~mL})$ was added IrCl(CO)( $\left.\mathrm{PPh}_{3}\right)_{2}(11.7 \mathrm{mg}, 0.015 \mathrm{mmol}, 0.1$ equiv) and toluene solution of TMDS ( $54 \mu \mathrm{L} / 4 \mathrm{~mL}$ (toluene), $0.3 \mathrm{mmol}, 2$ equiv) dropwise (4 mL / 1 hour) at room temperature. After 1 hours, the resulting crude residue was dissolved in ethyl acetate $(10 \mathrm{~mL})$ and saturated aqueous sodium bicarbonate solution $(10$ $\mathrm{mL}$ ) and the layers were separated. The aqueous layer was extracted with ethyl acetate $(3 \times 10 \mathrm{~mL})$, and the combined organic layers were dried over anhydrous sodium sulfate and concentrated under reduced pressure. The resulting crude residue was purified by column chromatography (silica gel: diam. $2 \mathrm{~cm}$, ht. $11 \mathrm{~cm}$; eluent : hexane : ethyl acetate $=3: 2$ to ethyl acetate) to afford $\mathbf{S 1}(22.3 \mathrm{mg}, 54 \%)$ as a white solid.

${ }^{1} \mathrm{H}$ NMR $\left(500 \mathrm{MHz}, \mathrm{CDCl}_{3}\right): \delta 6.64(\mathrm{dd}, \mathrm{J}=10.1,2.3 \mathrm{~Hz}, 1 \mathrm{H}), 6.08(\mathrm{dt}, \mathrm{J}=10.1,1.8 \mathrm{~Hz}, 1 \mathrm{H}), 5.87(\mathrm{~s}$, $1 \mathrm{H}$ ), 5.52 (ddd, $\mathrm{J}=10.3,5.6,2.7 \mathrm{~Hz}, 1 \mathrm{H}$ ), 3.01 (ddt, $\mathrm{J}=13.1,4.2,2.0 \mathrm{~Hz}, 1 \mathrm{H}$ ), 2.90 (ddd, J = 12.3, 6.0, $1.3 \mathrm{~Hz}, 1 \mathrm{H}), 2.68-2.62(\mathrm{~m}, 1 \mathrm{H}), 2.44(\mathrm{td}, \mathrm{J}=12.8,2.8 \mathrm{~Hz}, 1 \mathrm{H}), 2.06(\mathrm{~s}, 3 \mathrm{H}), 1.97-1.89(\mathrm{~m}, 2 \mathrm{H}), 1.75$ $(\mathrm{dd}, \mathrm{J}=12.3,10.3 \mathrm{~Hz}, 1 \mathrm{H}), 1.54-1.49(\mathrm{~m}, 1 \mathrm{H}), 1.49-1.39(\mathrm{~m}, 2 \mathrm{H}), 1.28-1.20(\mathrm{~m}, 2 \mathrm{H})$.

${ }^{13} \mathrm{C}$ NMR $\left(151 \mathrm{MHz}, \mathrm{CDCl}_{3}\right): \delta 172.3,170.4,164.3,136.4,122.6,113.4,87.9,67.8,58.6,47.3,37.3$, 27.3, 26.9, 25.1, 21.1.

HRMS (ESI): Calculated for $\mathrm{C}_{15} \mathrm{H}_{19} \mathrm{NO}_{4}[\mathrm{M}+\mathrm{H}]^{+}: 278.1387$, found: 278.1387 .

$[\alpha]^{25} \mathrm{D}:-3^{\circ}\left(c=1\right.$ in $\left.\mathrm{CHCl}_{3}\right)$.

TLC (hexane : acetone $=1: 1)$ Rf: $0.2(\mathrm{UV})$. 
<smiles>CC(=O)O[C@H]1C=CC2=CC(=O)O[C@]2(C2CCCCN2)C1</smiles>

s1

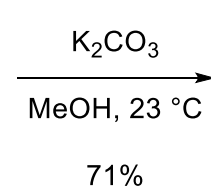

(-)-secu'amamine E (2)

\section{(-)-secu'amamine E (2):}

To a stirred solution of $\mathbf{S} 1$ (17.2 $\mathrm{mg}, 0.062 \mathrm{mmol}, 1$ equiv) in methanol $\left(2 \mathrm{~mL}\right.$ ) was added $\mathrm{K}_{2} \mathrm{CO}_{3}$ (42.9 mg, $0.3101 \mathrm{mmol}, 5$ equiv) at room temperature. After 1 hours, the crude product was diluted with dichloromethane $(10 \mathrm{~mL})$ and saturated aqueous sodium chloride solution $(10 \mathrm{~mL})$ and the layers were separated. The aqueous layer was extracted with dichloromethane $(3 \times 10 \mathrm{~mL})$, and the combined organic layers were dried over anhydrous sodium sulfate and concentrated under reduced pressure. The resulting crude residue was purified by column chromatography (silica gel: diam. $2 \mathrm{~cm}$, ht. $9 \mathrm{~cm}$; eluent : hexane : ethyl acetate $=1: 1)$ to afford $(-)$-secu'amamine $E(2)(10.3 \mathrm{mg}, 71 \%)$ as a white solid.

${ }^{1} \mathrm{H}$ NMR $\left(400 \mathrm{MHz}\right.$, Methanol- $\left.d_{4}\right): \delta 5.74$ (t, J = $\left.2.0 \mathrm{~Hz}, 1 \mathrm{H}\right), 4.30$ (dddd, J = 9.6, 4.7, 3.1, $1.4 \mathrm{~Hz}, 1 \mathrm{H}$ ), $3.01-2.95(\mathrm{~m}, 1 \mathrm{H}), 2.95-2.92(\mathrm{~m}, 1 \mathrm{H}), 2.89(\mathrm{td}, \mathrm{J}=3.5,1.3 \mathrm{~Hz}, 1 \mathrm{H}), 2.84-2.80(\mathrm{~m}, 1 \mathrm{H}), 2.78(\mathrm{dd}$, $\mathrm{J}=4.1,1.8 \mathrm{~Hz}, 1 \mathrm{H}), 2.74(\mathrm{dd}, \mathrm{J}=10.8,3.3 \mathrm{~Hz}, 1 \mathrm{H}), 2.67(\mathrm{dd}, \mathrm{J}=12.2,9.6 \mathrm{~Hz}, 1 \mathrm{H}), 1.86-1.76(\mathrm{~m}$, $1 \mathrm{H}), 1.62-1.56(\mathrm{~m}, 1 \mathrm{H}), 1.56-1.52(\mathrm{~m}, 1 \mathrm{H}), 1.51-1.45(\mathrm{~m}, 1 \mathrm{H}), 1.38(\mathrm{dd}, \mathrm{J}=12.2,4.9 \mathrm{~Hz}, 1 \mathrm{H}), 1.35$ $-1.28(\mathrm{~m}, 1 \mathrm{H}), 0.87(\mathrm{qd}, \mathrm{J}=12.0,4.1 \mathrm{~Hz}, 1 \mathrm{H})$.

${ }^{13} \mathrm{C}$ NMR (151 MHz, Methanol- $\left.d_{4}\right): \delta 177.2,176.3,112.0,86.2,66.4,65.3,60.3,53.5,41.5,30.3,27.7$, 26.7, 25.1 .

HRMS (ESI): Calculated for $\mathrm{C}_{13} \mathrm{H}_{17} \mathrm{NO}_{3}[\mathrm{M}+\mathrm{H}]^{+}: 236.1281$, found: 236.1282 .

$[\alpha]^{25} \mathrm{D}:-55^{\circ}(c=1$ in $\mathrm{MeOH}) .^{3}$

TLC (EA) Rf: $0.5\left(\mathrm{KMnO}_{4}\right)$. 


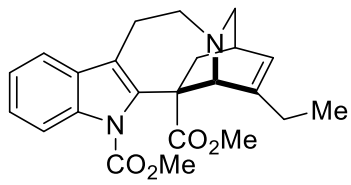

S2

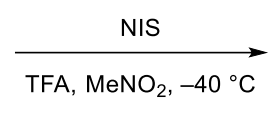

$38 \%$

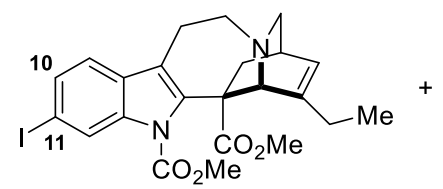

22

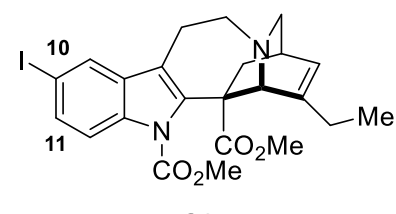

S3

$38 \%$

\section{C11-iodide 22:}

To a stirred solution of $\mathbf{S 2}^{4}\left(4.58 \mathrm{~g}, 11.61 \mathrm{mmol}, 1\right.$ equiv) in TFA (30 mL) and $\mathrm{MeNO}_{2}(30 \mathrm{~mL})$ was added $\mathrm{N}$-iodosuccinimide $\left(3.92 \mathrm{~g}, 17.41 \mathrm{mmol}, 1.5\right.$ equiv) at $-40^{\circ} \mathrm{C}$. After 12 hours, the resulting reaction mixture was diluted with ethyl acetate $(40 \mathrm{~mL})$ and saturated aqueous sodium thiosulfate solution $(50 \mathrm{~mL})$ and saturated aqueous sodium carbonate solution $(100 \mathrm{~mL})$ and the layers were separated. The aqueous layer was extracted with ethyl acetate $(3 \times 30 \mathrm{~mL})$, and the combined organic layers were dried over anhydrous sodium sulfate and concentrated under reduced pressure. The resulting crude residue was purified by column chromatography (silica gel: diam. $5 \mathrm{~cm}$, ht. $10 \mathrm{~cm}$; eluent : hexane : ethyl acetate $=3: 1)$ to afford $22(1.98 \mathrm{~g}, 38 \%)$ as a pale yellow solid and $\mathbf{S 3}(10-\mathrm{I})(1.98 \mathrm{~g}$, $38 \%)$ as a pale yellow solid.

${ }^{1} \mathrm{H}$ NMR $\left(500 \mathrm{MHz}, \mathrm{CDCl}_{3}\right): \delta 8.46(\mathrm{~d}, \mathrm{~J}=1.5 \mathrm{~Hz}, 1 \mathrm{H}), 7.55(\mathrm{dd}, \mathrm{J}=8.3,1.5 \mathrm{~Hz}, 1 \mathrm{H}), 7.21(\mathrm{~d}, \mathrm{~J}=8.3$ $\mathrm{Hz}, 1 \mathrm{H}), 5.97(\mathrm{dd}, \mathrm{J}=6.3,1.8 \mathrm{~Hz}, 1 \mathrm{H}), 4.18(\mathrm{~s}, 1 \mathrm{H}), 3.87(\mathrm{~s}, 3 \mathrm{H}), 3.62(\mathrm{ddd}, \mathrm{J}=11.8,5.0,2.6 \mathrm{~Hz}, 1 \mathrm{H}$ ), $3.52(\mathrm{~s}, 3 \mathrm{H}), 3.20$ (ddd, J = 15.6, 13.2, $5.0 \mathrm{~Hz}, 1 \mathrm{H}$ ), 3.02 (dt, J = 8.3, 3.0 Hz, 1H), $2.95-2.79(\mathrm{~m}, 2 \mathrm{H})$, $2.67-2.60(\mathrm{~m}, 2 \mathrm{H}), 2.45(\mathrm{~d}, \mathrm{~J}=8.2 \mathrm{~Hz}, 1 \mathrm{H}), 2.22(\mathrm{dqd}, \mathrm{J}=16.8,7.2,2.2 \mathrm{~Hz}, 1 \mathrm{H}), 1.88(\mathrm{dqd}, \mathrm{J}=16.9$, 7.3, $2.1 \mathrm{~Hz}, 1 \mathrm{H}), 1.71-1.64(\mathrm{~m}, 1 \mathrm{H}), 1.06(\mathrm{t}, \mathrm{J}=7.3 \mathrm{~Hz}, 3 \mathrm{H})$.

${ }^{13} \mathrm{C}$ NMR $\left(101 \mathrm{MHz}, \mathrm{CDCl}_{3}\right): \delta 172.9,151.7,147.5,139.2,137.0,131.9,129.2,124.6,123.6,120.0$, $119.7,89.3,58.8,56.1,56.1,53.6,52.9,52.4,38.4,31.7,27.0,22.1,10.6$.

HRMS (ESI): Calculated for $\mathrm{C}_{23} \mathrm{H}_{25} \mathrm{IN}_{2} \mathrm{O}_{4}[\mathrm{M}+\mathrm{H}]^{+}: 521.0932$, found: 521.0928.

$[\alpha]^{25} \mathrm{D}:+46^{\circ}\left(C=1\right.$ in $\left.\mathrm{CHCl}_{3}\right)$. TLC (hexane : ethyl acetate $\left.=1: 1\right)$ Rf: 0.15 (CAM, UV).

\section{C10-iodide S3:}

${ }^{1} \mathrm{H}$ NMR $\left(400 \mathrm{MHz}, \mathrm{CDCl}_{3}\right): \delta 7.84(\mathrm{~d}, \mathrm{~J}=8.8 \mathrm{~Hz}, 1 \mathrm{H}), 7.79(\mathrm{~d}, \mathrm{~J}=1.8 \mathrm{~Hz}, 1 \mathrm{H}), 7.55(\mathrm{dd}, \mathrm{J}=8.8,1.8$ $\mathrm{Hz}, 1 \mathrm{H}), 5.97(\mathrm{~d}, \mathrm{~J}=6.2 \mathrm{~Hz}, 1 \mathrm{H}), 4.18(\mathrm{~s}, 1 \mathrm{H}), 3.86(\mathrm{~s}, 3 \mathrm{H}), 3.68-3.59(\mathrm{~m}, 1 \mathrm{H}), 3.53(\mathrm{~s}, 3 \mathrm{H}), 3.19$ (ddd, $\mathrm{J}=16.5,13.6,4.9 \mathrm{~Hz}, 1 \mathrm{H}), 3.03(\mathrm{dt}, \mathrm{J}=8.5,2.9 \mathrm{~Hz}, 1 \mathrm{H}), 2.94-2.77(\mathrm{~m}, 2 \mathrm{H}), 2.70-2.59(\mathrm{~m}, 2 \mathrm{H})$, $2.45(\mathrm{~d}, \mathrm{~J}=8.1 \mathrm{~Hz}, 1 \mathrm{H}), 2.23(\mathrm{dtd}, \mathrm{J}=15.5,7.8,5.7 \mathrm{~Hz}, 1 \mathrm{H}), 1.95-1.80(\mathrm{~m}, 1 \mathrm{H}), 1.73-1.63(\mathrm{~m}, 1 \mathrm{H})$, $1.06(\mathrm{t}, \mathrm{J}=7.3 \mathrm{~Hz}, 3 \mathrm{H})$.

${ }^{13} \mathrm{C}$ NMR $\left(101 \mathrm{MHz}, \mathrm{CDCl}_{3}\right): \delta 172.9,151.7,147.5,139.7,135.5,133.2,132.1,127.5,123.6,118.9$, $117.6,86.9,58.8,56.1,56.1,53.5,52.8,52.4,38.5,31.7,27.0,22.1,10.6$.

HRMS (ESI): Calculated for $\mathrm{C}_{23} \mathrm{H}_{25} \mathrm{IN}_{2} \mathrm{O}_{4}[\mathrm{M}+\mathrm{H}]^{+}: 521.0932$, found: 521.0932.

$[\alpha]^{25} \mathrm{D}:+35^{\circ}\left(c=1\right.$ in $\left.\mathrm{CHCl}_{3}\right)$. TLC (hexane : ethyl acetate $\left.=1: 1\right) \mathrm{R} f: 0.3$ (CAM, UV). 
<smiles>CCC1=CN2CCC1C1(C(=O)OC)CCN2CCc2c1n(C(C)=O)c1cc(I)ccc21</smiles>

22

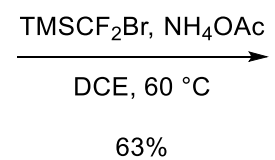

$63 \%$

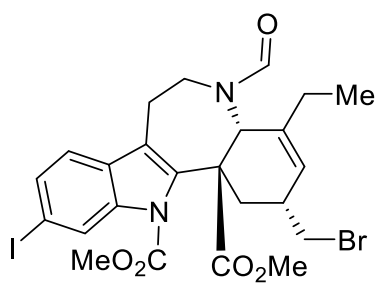

24

\section{bromide 24:}

To a stirred solution of $\mathrm{TMSCF}_{2} \mathrm{Br}\left(1 \mathrm{~mL}, 6.523 \mathrm{mmol}, 4\right.$ equiv) and $\mathrm{NH}_{4} \mathrm{OAc}(502.8 \mathrm{mg}, 6.523$ mmol, 4 equiv) in DCE (8 mL) was added a solution of $22(848.6 \mathrm{mg}, 1.6308 \mathrm{mmol}, 1$ equiv) in DCE (16 $\mathrm{mL}$ ) and stirring at room temperature. After 20 minutes, the temperature was raised to $60^{\circ} \mathrm{C}$. After 15 hours, the reaction mixture was cooled to $23^{\circ} \mathrm{C}$ and was dissolved in dichloromethane $(30 \mathrm{~mL})$ and saturated aqueous sodium chloride solution $(30 \mathrm{~mL})$ and the layers were separated. The aqueous layer was extracted with dichloromethane $(3 \times 20 \mathrm{~mL})$, and the combined organic layers were dried over anhydrous sodium sulfate and concentrated under reduced pressure. The resulting crude residue was purified by column chromatography (silica gel: diam. $3 \mathrm{~cm}$, ht. $10 \mathrm{~cm}$; eluent : hexane : ethyl acetate = $3: 2)$ to afford $24(590.2 \mathrm{mg}, 63 \%)$ as a white solid.

${ }^{1} \mathrm{H}$ NMR $\left(500 \mathrm{MHz}, \mathrm{CDCl}_{3}\right): \delta 8.40(\mathrm{~d}, \mathrm{~J}=1.4 \mathrm{~Hz}, 1 \mathrm{H}), 8.02(\mathrm{~s}, 1 \mathrm{H}), 7.58(\mathrm{dd}, \mathrm{J}=8.3,1.5 \mathrm{~Hz}, 1 \mathrm{H}), 7.16$ $(\mathrm{d}, \mathrm{J}=8.3 \mathrm{~Hz}, 1 \mathrm{H}), 5.46(\mathrm{~s}, 1 \mathrm{H}), 4.67(\mathrm{~s}, 1 \mathrm{H}), 4.03(\mathrm{~s}, 3 \mathrm{H}), 3.82-3.76(\mathrm{~m}, 1 \mathrm{H}), 3.68-3.61(\mathrm{~m}, 1 \mathrm{H})$, $3.58(\mathrm{~s}, 3 \mathrm{H}), 3.03-2.88(\mathrm{~m}, 3 \mathrm{H}), 2.78-2.67(\mathrm{~m}, 1 \mathrm{H}), 2.64-2.57(\mathrm{~m}, 2 \mathrm{H}), 2.15(\mathrm{dt}, \mathrm{J}=15.0,7.6 \mathrm{~Hz}$, $1 \mathrm{H}), 2.06(\mathrm{dt}, \mathrm{J}=15.6,7.3 \mathrm{~Hz}, 1 \mathrm{H}), 1.63(\mathrm{~s}, 1 \mathrm{H}), 1.04(\mathrm{t}, \mathrm{J}=7.4 \mathrm{~Hz}, 3 \mathrm{H})$.

${ }^{13} \mathrm{C}$ NMR $\left(126 \mathrm{MHz}, \mathrm{CDCl}_{3}\right): \delta 174.7,163.7,152.3,140.9,136.5,134.0,132.6,128.6,125.6,122.9$, $121.3,119.9,90.5,64.0,54.6,53.1,52.9,45.0,36.9,34.9,31.9,27.3,19.1,12.9$.

HRMS (ESI): Calculated for $\mathrm{C}_{24} \mathrm{H}_{26} \mathrm{BrIN}_{2} \mathrm{O}_{5}[\mathrm{M}+\mathrm{Na}]^{+}: 650.9962$, found: 650.9967 .

$[\alpha]^{25} \mathrm{D}:+162^{\circ}\left(c=1\right.$ in $\left.\mathrm{CHCl}_{3}\right)$.

TLC (hexane : ethyl acetate $=1: 1$ ) Rf: 0.4 (UV) 
<smiles>CCC1=C[C@@H](CBr)C[C@]2(C(C)=O)c3c(c4ccc(I)cc4n3C)CCN(C=O)[C@H]12</smiles>

24

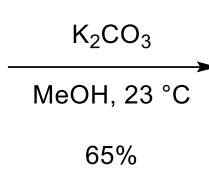

$65 \%$

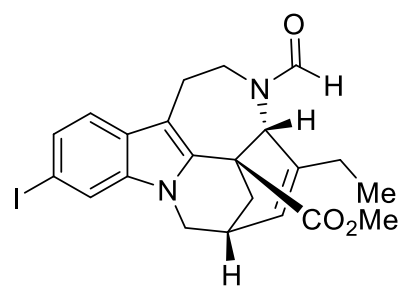

25

\section{pentacycle 25:}

To a solution of 24 (604.9 mg, $1.0589 \mathrm{mmol}, 1$ equiv) in $\mathrm{MeOH}(10 \mathrm{~mL})$ was added $\mathrm{K}_{2} \mathrm{CO}_{3}$ (731.7mg, $5.2945 \mathrm{mmol}, 5$ equiv). After 2 hours, the resulting reaction mixture was diluted with dichloromethane $(10 \mathrm{~mL})$ and saturated aqueous sodium chloride solution $(15 \mathrm{~mL})$ and the layers were separated. The aqueous layer was extracted with dichloromethane $(3 \times 10 \mathrm{~mL})$, and the combined organic layers were dried over anhydrous sodium sulfate and concentrated under reduced pressure. The resulting crude residue was purified by column chromatography (silica gel: diam. $2 \mathrm{~cm}$, ht. $9 \mathrm{~cm}$; eluent : hexane : ethyl acetate $=3: 2)$ to afford $25(339 \mathrm{mg}, 65 \%)$ as a yellow solid.

${ }^{1} \mathrm{H}$ NMR $\left(500 \mathrm{MHz}, \mathrm{CDCl}_{3}\right): \delta 8.33(\mathrm{~s}, 1 \mathrm{H}), 7.59(\mathrm{~s}, 1 \mathrm{H}), 7.37(\mathrm{dd}, \mathrm{J}=8.3,1.5 \mathrm{~Hz}, 1 \mathrm{H}), 7.22(\mathrm{~d}, \mathrm{~J}=8.4$ $\mathrm{Hz}, 1 \mathrm{H}), 5.43-5.41(\mathrm{~m}, 1 \mathrm{H}), 4.74(\mathrm{~s}, 1 \mathrm{H}), 4.57$ (ddd, $\mathrm{J}=13.3,4.6,1.7 \mathrm{~Hz}, 1 \mathrm{H}), 4.12$ (dd, J = 11.2, 4.0 $\mathrm{Hz}, 1 \mathrm{H}), 3.91(\mathrm{dt}, \mathrm{J}=11.3,1.8 \mathrm{~Hz}, 1 \mathrm{H}), 3.71(\mathrm{~s}, 3 \mathrm{H}), 2.92(\mathrm{~d}, \mathrm{~J}=4.8 \mathrm{~Hz}, 1 \mathrm{H}), 2.84(\mathrm{dd}, \mathrm{J}=15.6,4.6$ $\mathrm{Hz}, 1 \mathrm{H}$ ), 2.53 (ddd, $\mathrm{J}=15.7,11.7,1.8 \mathrm{~Hz}, 1 \mathrm{H}), 2.41$ (ddd, $\mathrm{J}=12.7,4.9,1.8 \mathrm{~Hz}, 1 \mathrm{H}), 2.31(\mathrm{~d}, \mathrm{~J}=12.6$ $\mathrm{Hz}, 1 \mathrm{H}), 2.19(\mathrm{t}, \mathrm{J}=12.3 \mathrm{~Hz}, 1 \mathrm{H}), 1.69(\mathrm{q}, \mathrm{J}=7.4 \mathrm{~Hz}, 2 \mathrm{H}), 0.88(\mathrm{t}, \mathrm{J}=7.4 \mathrm{~Hz}, 3 \mathrm{H})$.

${ }^{13} \mathrm{C} \mathrm{NMR}\left(126 \mathrm{MHz}, \mathrm{CDCl}_{3}\right) \delta 173.1,165.4,142.5,138.0,134.0,128.7,126.6,125.1,120.1,118.9$, $114.9,85.2,62.6,53.2,49.5,48.3,41.8,35.0,30.9,26.1,24.4,12.9$.

HRMS (ESI): Calculated for $\mathrm{C}_{22} \mathrm{H}_{23} \mathrm{IN}_{2} \mathrm{O}_{3}[\mathrm{M}+\mathrm{Na}]^{+}: 513.0646$, found: 513.0645 .

$[\alpha]^{25} \mathrm{D}:+99^{\circ}\left(c=1\right.$ in $\left.\mathrm{CHCl}_{3}\right)$.

TLC (hexane : ethyl acetate $=1: 1)$ Rf: 0.3 (UV). 


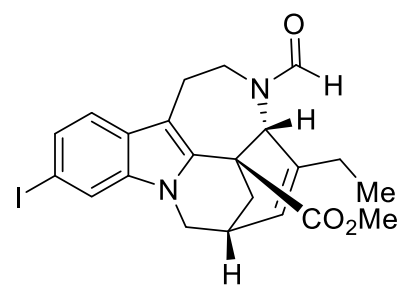

25

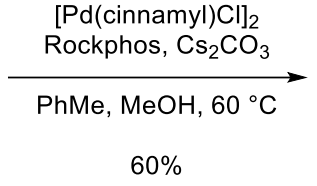

$60 \%$

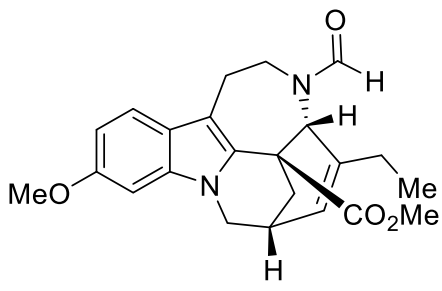

S4

\section{methoxyindole S4:}

To a solution of 25 (121.6 mg, $0.2480 \mathrm{mmol}, 1$ equiv) in toluene $(6 \mathrm{~mL})$ was added [Pd(cinnamyl)Cl $]_{2}$ (39 mg, $0.0744 \mathrm{mmol}, 0.3$ equiv), Rockphos (70 mg, $0.1488 \mathrm{mmol}, 0.6$ equiv), $\mathrm{Cs}_{2} \mathrm{CO}_{3}$ (202 mg, $0.62 \mathrm{mmol}, 2.5$ equiv) and methanol ( $0.1 \mathrm{~mL}, 2.480 \mathrm{mmol}, 10.0$ equiv) in glove box and raised temperature to $60^{\circ} \mathrm{C}$. After 12 hours, the crude product was filtered through a pad of celite, washed with ethyl acetate $(10 \mathrm{~mL})$ and the resulting mixture was diluted with ethyl acetate $(10 \mathrm{~mL})$ and saturated aqueous sodium chloride solution $(10 \mathrm{~mL})$ and the layers were separated. The aqueous layer was extracted with ethyl acetate $(3 \times 10 \mathrm{~mL})$, and the combined organic layers were dried over anhydrous sodium sulfate and concentrated under reduced pressure. The resulting crude residue was purified by column chromatography (silica gel: diam. $3 \mathrm{~cm}$, ht. $9 \mathrm{~cm}$; eluent : hexane : ethyl acetate = $3: 1)$ to afford $\mathbf{S} 4(58.3 \mathrm{mg}, 60 \%)$ as a yellow solid.

${ }^{1} \mathrm{H}$ NMR $\left(500 \mathrm{MHz}, \mathrm{CDCl}_{3}\right): \delta 8.34(\mathrm{~s}, 1 \mathrm{H}), 7.34(\mathrm{~d}, \mathrm{~J}=8.7 \mathrm{~Hz}, 1 \mathrm{H}), 6.78(\mathrm{dd}, \mathrm{J}=8.6,2.3 \mathrm{~Hz}, 1 \mathrm{H}), 6.71$ $(\mathrm{d}, \mathrm{J}=2.3 \mathrm{~Hz}, 1 \mathrm{H}), 5.42(\mathrm{dd}, \mathrm{J}=3.6,2.0 \mathrm{~Hz}, 1 \mathrm{H}), 4.74(\mathrm{~s}, 1 \mathrm{H}), 4.56(\mathrm{dd}, \mathrm{J}=13.2,3.3 \mathrm{~Hz}, 1 \mathrm{H}), 4.12$ (dd, J = 11.1, $4.1 \mathrm{~Hz}, 1 \mathrm{H}), 3.89(\mathrm{~d}, \mathrm{~J}=11.0 \mathrm{~Hz}, 1 \mathrm{H}), 3.85(\mathrm{~s}, 3 \mathrm{H}), 3.71(\mathrm{~s}, 3 \mathrm{H}), 2.91(\mathrm{~d}, \mathrm{~J}=4.5 \mathrm{~Hz}, 1 \mathrm{H})$, $2.84(\mathrm{dd}, \mathrm{J}=15.5,4.5 \mathrm{~Hz}, 1 \mathrm{H}), 2.54$ (ddd, $\mathrm{J}=15.5,11.5,1.7 \mathrm{~Hz}, 1 \mathrm{H}), 2.42-2.37(\mathrm{~m}, 1 \mathrm{H}), 2.34-2.30$ (m, 1H), $2.23(\mathrm{t}, \mathrm{J}=12.2 \mathrm{~Hz}, 1 \mathrm{H}), 1.69(\mathrm{q}, \mathrm{J}=7.3 \mathrm{~Hz}, 2 \mathrm{H}), 0.88(\mathrm{t}, \mathrm{J}=7.3 \mathrm{~Hz}, 3 \mathrm{H})$.

${ }^{13} \mathrm{C} \mathrm{NMR}\left(126 \mathrm{MHz}, \mathrm{CDCl}_{3}\right) \delta 173.6,165.4,156.3,142.3,137.4,132.0,125.3,121.8,119.0,114.5$, 109.6, 93.6, 62.7, 56.0, 53.1, 49.5, 48.4, 41.9, 35.0, 31.0, 26.2, 24.5, 12.9 .

HRMS (ESI): Calculated for $\mathrm{C}_{23} \mathrm{H}_{26} \mathrm{~N}_{2} \mathrm{O}_{4}[\mathrm{M}+\mathrm{Na}]^{+}: 417.1785$, found: 417.1785 .

$[\alpha]^{25} \mathrm{D}:+83^{\circ}\left(c=1\right.$ in $\left.\mathrm{CHCl}_{3}\right)$.

TLC (hexane : ethyl acetate $=1: 1$ ) Rf: 0.25 (UV). 


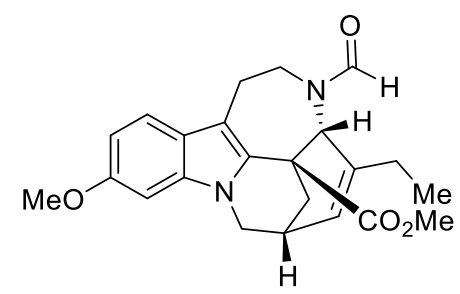

S4

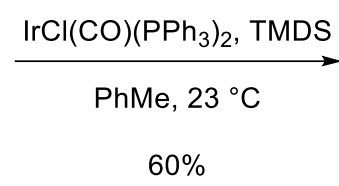

$60 \%$

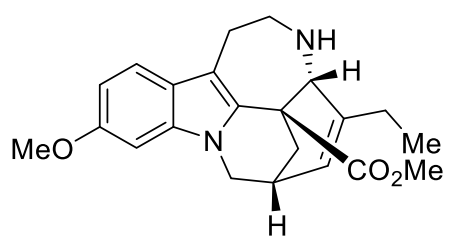

26

\section{amine 26:}

To a solution of $\mathbf{S 4}(303.6 \mathrm{mg}, 0.7696 \mathrm{mmol}, 1$ equiv) in toluene $(15 \mathrm{~mL})$ was added $\operatorname{IrCl}(\mathrm{CO})\left(\mathrm{PPh}_{3}\right)_{2}(60 \mathrm{mg}, 0.07696 \mathrm{mmol}, 0.1$ equiv) and toluene solution of TMDS (136 $\mu \mathrm{L} / 9 \mathrm{~mL}$ (toluene), $0.7696 \mathrm{mmol}, 1$ equiv) dropwise (9 mL / 1 hour) at room temperature. After 1 hours, the resulting crude residue was dissolved in ethyl acetate $(10 \mathrm{~mL})$ and saturated aqueous sodium bicarbonate solution (10 $\mathrm{mL})$ and the layers were separated. The aqueous layer was extracted with ethyl acetate $(3 \times 10 \mathrm{~mL})$, and the combined organic layers were dried over anhydrous sodium sulfate and concentrated under reduced pressure. The resulting crude residue was purified by column chromatography (silica gel: diam. $2 \mathrm{~cm}$, ht. $11 \mathrm{~cm}$; eluent : hexane : ethyl acetate $=3: 2)$ to afford $26(168.6 \mathrm{mg}, 60 \%)$ as a yellow solid.

${ }^{1} \mathrm{H}$ NMR $\left(500 \mathrm{MHz}, \mathrm{CDCl}_{3}\right): \delta 7.29(\mathrm{~d}, \mathrm{~J}=8.6 \mathrm{~Hz}, 1 \mathrm{H}), 6.75(\mathrm{dd}, \mathrm{J}=8.5,2.3 \mathrm{~Hz}, 1 \mathrm{H}), 6.70(\mathrm{~d}, \mathrm{~J}=2.3$ $\mathrm{Hz}, 1 \mathrm{H}), 5.31-5.29(\mathrm{~m}, 1 \mathrm{H}), 4.09(\mathrm{~s}, 1 \mathrm{H}), 4.07(\mathrm{dd}, \mathrm{J}=11.1,4.1 \mathrm{~Hz}, 1 \mathrm{H}), 3.87(\mathrm{~s}, 1 \mathrm{H}), 3.84(\mathrm{~s}, 3 \mathrm{H})$, $3.74(\mathrm{~s}, 3 \mathrm{H}), 2.96$ (ddd, J = 13.9, 4.7, $2.7 \mathrm{~Hz}, 1 \mathrm{H}), 2.80(\mathrm{~s}, 1 \mathrm{H}), 2.74$ (ddd, J = 15.2, 4.7, $2.5 \mathrm{~Hz}, 1 \mathrm{H}$ ), 2.61 (ddd, J = 15.2, 10.3, $2.7 \mathrm{~Hz}, 1 \mathrm{H}), 2.52-2.43(\mathrm{~m}, 1 \mathrm{H}), 2.24-2.22(\mathrm{~m}, 2 \mathrm{H}), 2.04(\mathrm{dq}, \mathrm{J}=14.6,7.2$ $\mathrm{Hz}, 1 \mathrm{H}), 1.81(\mathrm{dq}, \mathrm{J}=14.8,7.2 \mathrm{~Hz}, 1 \mathrm{H}), 0.93(\mathrm{t}, \mathrm{J}=7.4 \mathrm{~Hz}, 3 \mathrm{H})$.

${ }^{13} \mathrm{C}$ NMR $\left(151 \mathrm{MHz}, \mathrm{CDCl}_{3}\right): \delta 175.7,155.9,145.8,137.2,133.5,122.9,122.3,118.8,113.6,109.1$, 93.6, 58.7, 56.1, 52.7, 50.9, 48.1, 44.5, 34.8, 31.0, 27.3, 26.7, 13.2 .

HRMS (ESI): Calculated for $\mathrm{C}_{22} \mathrm{H}_{26} \mathrm{~N}_{2} \mathrm{O}_{3}[\mathrm{M}+\mathrm{H}]^{+}: 367.2016$, found: 367.2019 .

$[\alpha]^{25} \mathrm{D}:+55^{\circ}\left(c=1\right.$ in $\left.\mathrm{CHCl}_{3}\right)$.

TLC (hexane : ethyl acetate $=1: 1)$ Rf: $0.3(U V)$. 


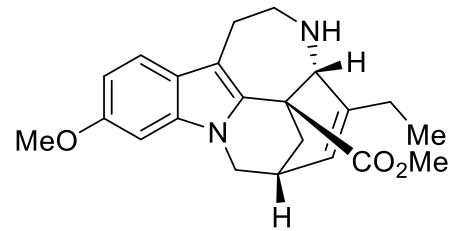

26

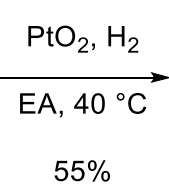

$55 \%$<smiles>CC[C@H]1[C@@H](COC)Cn2c3c(c4ccc(OC)cc42)[C@@H]1NCC3</smiles>

27

\section{purported (+)-tabercarpamine G (27):}

To a solution of 26 (29.6 mg, 0.0808 mmol, 1 equiv) in ethyl acetate $(5 \mathrm{~mL})$ was added $\mathrm{PtO}_{2}$ (18.3 $\mathrm{mg}, 0.0808 \mathrm{mmol}, 1$ equiv) at room temperature. The reaction flask was evacuated and filled with $\mathrm{H}_{2}$. The reaction mixture was stirred at $40{ }^{\circ} \mathrm{C}$. After 16 hours, the crude reaction mixture was filtered through a pad of celite, washed with ethyl acetate $(3 \times 10 \mathrm{~mL})$ and concentrated under reduced pressure. The resulting crude residue was purified by column chromatography (silica gel: diam. $2 \mathrm{~cm}$, ht. $8 \mathrm{~cm}$; eluent : hexane : ethyl acetate $=1: 1)$ to afford $27(16.3 \mathrm{mg}, 55 \%)$ as a yellow solid.

${ }^{1} \mathbf{H}$ NMR $\left(500 \mathrm{MHz}_{\mathrm{CDCl}}\right): \delta 7.38(\mathrm{~d}, \mathrm{~J}=8.6 \mathrm{~Hz}, 1 \mathrm{H}), 6.80(\mathrm{dd}, \mathrm{J}=8.6,2.3 \mathrm{~Hz}, 1 \mathrm{H}), 6.75(\mathrm{~d}, \mathrm{~J}=2.3$ $\mathrm{Hz}, 1 \mathrm{H}), 4.06(\mathrm{dd}, \mathrm{J}=11.0,3.4 \mathrm{~Hz}, 1 \mathrm{H}), 3.90(\mathrm{~d}, \mathrm{~J}=7.1 \mathrm{~Hz}, 1 \mathrm{H}), 3.86(\mathrm{~s}, 3 \mathrm{H}), 3.82-3.79(\mathrm{~m}, 1 \mathrm{H})$, $3.70(\mathrm{~s}, 3 \mathrm{H}), 3.20-3.13(\mathrm{~m}, 1 \mathrm{H}), 2.98-2.88(\mathrm{~m}, 1 \mathrm{H}), 2.72-2.61(\mathrm{~m}, 2 \mathrm{H}), 2.60-2.53(\mathrm{~m}, 1 \mathrm{H}), 2.24$ (ddd, J = 12.9, 5.7, 1.7 Hz, 1H), $1.92(\mathrm{~d}, \mathrm{~J}=12.9 \mathrm{~Hz}, 1 \mathrm{H}), 1.80$ (dqd, J = 14.2, 7.1, 3.4 Hz, $1 \mathrm{H}), 1.65$ (ddd, J = 13.9, 10.3, 3.4 Hz, 1H), $1.44-1.35(\mathrm{~m}, 1 \mathrm{H}), 1.00(\mathrm{tt}, \mathrm{J}=13.2,6.1 \mathrm{~Hz}, 1 \mathrm{H}), 0.92(\mathrm{t}, \mathrm{J}=7.2$ $\mathrm{Hz}, 3 \mathrm{H}), 0.50(\mathrm{td}, \mathrm{J}=14.1,6.8 \mathrm{~Hz}, 1 \mathrm{H})$.

${ }^{13} \mathrm{C}$ NMR $\left(151 \mathrm{MHz}, \mathrm{CDCl}_{3}\right): \delta 176.8,155.9,137.8,131.1,123.0,118.8,109.1,108.1,93.2,58.5,56.0$, 52.7, 49.9, 49.6, 40.8, 36.9, 30.5, 27.2, 26.0, 24.3, 23.2, 12.6.

HRMS (ESI): Calculated for $\mathrm{C}_{22} \mathrm{H}_{28} \mathrm{~N}_{2} \mathrm{O}_{3}[\mathrm{M}+\mathrm{H}]^{+}:$369.2173, found: 369.2176.

$[\alpha]^{25} \mathrm{D}:+99^{\circ}\left(c=1\right.$ in $\left.\mathrm{CHCl}_{3}\right)$.

TLC (hexane : ethyl acetate $=1: 1$ ) Rf: 0.15 (UV). 
<smiles>COC[C@H]1C[C@@H]2Cn3c4c(c5ccc(OC)cc53)C[C@@H]1[C@H]2NCC4</smiles>

27

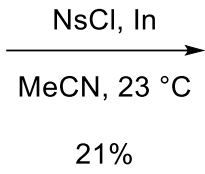

$21 \%$

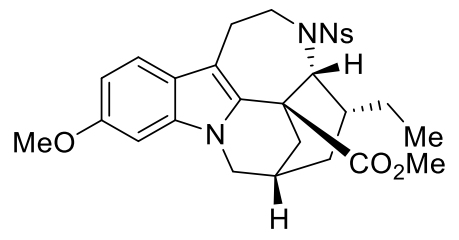

46

\section{sulfonylamide 46:}

To a solution of 27 (93.1 mg, $0.2527 \mathrm{mmol}, 1$ equiv) in MeCN (5 mL) was added 2nitrobenzenesulfonyl chloride ( $28 \mathrm{mg}, 1.2633 \mathrm{mmol}, 5$ equiv) and indium powder ( $145 \mathrm{mg}, 1.2633 \mathrm{mmol}$, 5 equiv). After 16 hours, the resulting crude residue was dissolved in ethyl acetate $(10 \mathrm{~mL})$ and saturated aqueous sodium bicarbonate solution $(10 \mathrm{~mL})$ and the layers were separated. The aqueous layer was extracted with ethyl acetate $(3 \times 10 \mathrm{~mL})$, and the combined organic layers were dried over anhydrous sodium sulfate and concentrated under reduced pressure. the reaction mixture was purified by column chromatography (silica gel: diam. $1 \mathrm{~cm}$, ht. $8 \mathrm{~cm}$; eluent : hexane: ethyl acetate $=2: 1$ ) to afford $46(29 \mathrm{mg}, 21 \%)$ as a yellow solid and 27 (36.8 $\mathrm{mg}, 40 \% \mathrm{RSM})$.

${ }^{1} \mathrm{H}$ NMR $\left(500 \mathrm{MHz}, \mathrm{CDCl}_{3}\right): \delta 8.12-8.07(\mathrm{~m}, 1 \mathrm{H}), 7.70-7.65(\mathrm{~m}, 2 \mathrm{H}), 7.61(\mathrm{dd}, \mathrm{J}=5.9,3.3 \mathrm{~Hz}, 1 \mathrm{H})$, $7.33(\mathrm{~d}, \mathrm{~J}=8.7 \mathrm{~Hz}, 1 \mathrm{H}), 6.78(\mathrm{dd}, \mathrm{J}=8.6,2.3 \mathrm{~Hz}, 1 \mathrm{H}), 6.70(\mathrm{~d}, \mathrm{~J}=2.2 \mathrm{~Hz}, 1 \mathrm{H}), 4.73(\mathrm{~d}, \mathrm{~J}=7.3 \mathrm{~Hz}$, $1 \mathrm{H}), 4.15(\mathrm{dd}, \mathrm{J}=11.7,5.4 \mathrm{~Hz}, 1 \mathrm{H}), 3.96(\mathrm{ddd}, \mathrm{J}=14.4,5.7,3.2 \mathrm{~Hz}, 1 \mathrm{H}), 3.85(\mathrm{~s}, 3 \mathrm{H}), 3.84-3.78(\mathrm{~m}$, 1H), 3.52 (s, 3H), 3.35 (ddd, J = 14.3, 9.6, 3.3 Hz, 1H), 2.97 (qdt, J = 9.1, 5.6, 3.2 Hz, 2H), $2.49(\mathrm{~s}, 1 \mathrm{H}$ ), 2.30 (ddt, J = 11.6, 7.5, 3.6 Hz, 1H), 2.25 (dt, J = 12.9, 2.4 Hz, 1H), 2.13 (ddd, J = 12.8, 4.6, $1.7 \mathrm{~Hz}$, $1 \mathrm{H}$ ), 1.95 (dt, $\mathrm{J}=14.0,6.6 \mathrm{~Hz}, 1 \mathrm{H}$ ), 1.58 (dq, $\mathrm{J}=15.1,3.1 \mathrm{~Hz}, 1 \mathrm{H}$ ), 0.95 (ddd, J = 13.6, 7.4, 3.2 Hz, $1 \mathrm{H}), 0.51$ (t, $\mathrm{J}=7.3 \mathrm{~Hz}, 3 \mathrm{H}$ ), -0.65 (ddq, $\mathrm{J}=14.1,11.6,7.1 \mathrm{~Hz}, 1 \mathrm{H}$ ).

${ }^{13} \mathrm{C}$ NMR $\left(100.6 \mathrm{MHz}, \mathrm{CDCl}_{3}\right): \delta 174.0,156.3,148.8,138.0,133.8,133.4,131.9,131.5,131.5,124.1$, 122.0, 119.4, 111.8, 109.7, 93.6, 61.4, 55.9, 52.8, 48.5, 47.3, 46.7, 40.5, 37.1, 31.4, 26.9, 24.5, 21.1, 13.9 .

HRMS (ESI): Calculated for $\mathrm{C}_{28} \mathrm{H}_{31} \mathrm{~N}_{3} \mathrm{O}_{7} \mathrm{~S}[\mathrm{M}+\mathrm{Na}]^{+}: 576.1775$, found: 576.1775 .

$[\alpha]^{25} \mathrm{D}:+130^{\circ}\left(\mathrm{C}=1\right.$ in $\left.\mathrm{CHCl}_{3}\right)$.

TLC (hexane : ethyl acetate $=1: 1)$ Rf: 0.3 (UV). 


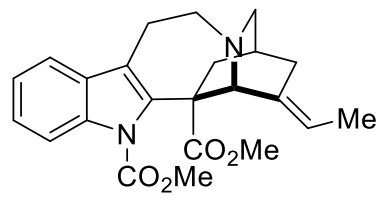

28

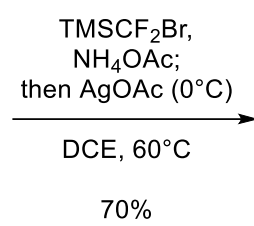

$70 \%$

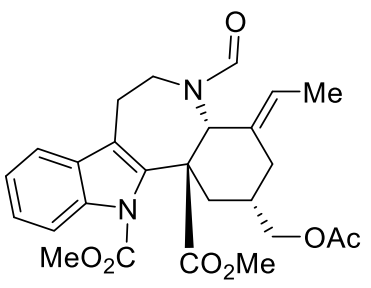

31

\section{acetate 31:}

To a stirred solution of $\mathrm{TMSCF}_{2} \mathrm{Br}\left(0.93 \mathrm{~mL}, 5.972 \mathrm{mmol}, 4\right.$ equiv) and $\mathrm{NH}_{4} \mathrm{OAc}$ (466.3 mg, $5.972 \mathrm{mmol}$, 4 equiv) in DCE (8 mL) was added a solution of $28^{5}(589 \mathrm{mg}, 1.493 \mathrm{mmol}, 1$ equiv) in DCE $(16 \mathrm{~mL})$ and stirring at room temperature. After 20 minutes, the reaction mixture was cooled to $0^{\circ} \mathrm{C}$ and was added AgOAc (996.8 mg, $5.972 \mathrm{mmol}, 4$ equiv). After 20 minutes, the temperature was raised to $60^{\circ} \mathrm{C}$. After 15 hours, the reaction mixture was cooled to $23^{\circ} \mathrm{C}$ and was dissolved in ethyl acetate (30 $\mathrm{mL}$ ) and saturated aqueous sodium chloride solution $(30 \mathrm{~mL})$ and the layers were separated. The aqueous layer was extracted with ethyl acetate $(3 \times 20 \mathrm{~mL})$, and the combined organic layers were dried over anhydrous sodium sulfate and concentrated under reduced pressure. The resulting crude residue was purified by column chromatography (silica gel: diam. $3 \mathrm{~cm}$, ht. $10 \mathrm{~cm}$; eluent : hexane : ethyl acetate $=2: 3)$ to afford $31(504 \mathrm{mg}, 70 \%)$ as a white solid.

${ }^{1} \mathrm{H}$ NMR $\left(400 \mathrm{MHz}, \mathrm{CDCl}_{3}\right): \delta 7.99(\mathrm{~s}, 1 \mathrm{H}), 7.98(\mathrm{~d}, \mathrm{~J}=8.0 \mathrm{~Hz}, 1 \mathrm{H}), 7.42(\mathrm{~d}, \mathrm{~J}=7.3 \mathrm{~Hz}, 1 \mathrm{H}), 7.36-$ $7.28(\mathrm{~m}, 1 \mathrm{H}), 7.27(\mathrm{td}, \mathrm{J}=7.5,1.2 \mathrm{~Hz}, 1 \mathrm{H}), 5.57(\mathrm{~d}, \mathrm{~J}=8.2 \mathrm{~Hz}, 1 \mathrm{H}), 4.66(\mathrm{~s}, 1 \mathrm{H}), 3.99(\mathrm{~d}, \mathrm{~J}=2.8 \mathrm{~Hz}$, $1 \mathrm{H}), 3.97(\mathrm{~s}, 3 \mathrm{H}), 3.70-3.59(\mathrm{~m}, 3 \mathrm{H}), 3.57(\mathrm{~s}, 3 \mathrm{H}), 3.25(\mathrm{ddd}, \mathrm{J}=17.7,13.8,4.0 \mathrm{~Hz}, 1 \mathrm{H}), 2.97(\mathrm{ddt}, \mathrm{J}$ $=16.4,2.7,1.3 \mathrm{~Hz}, 1 \mathrm{H}), 2.93-2.85(\mathrm{~m}, 1 \mathrm{H}), 2.43(\mathrm{dd}, \mathrm{J}=15.5,4.5 \mathrm{~Hz}, 1 \mathrm{H}), 2.22$ (ddt, $\mathrm{J}=13.9,6.7$, $3.4 \mathrm{~Hz}, 1 \mathrm{H}), 2.14(\mathrm{~d}, \mathrm{~J}=10.2 \mathrm{~Hz}, 1 \mathrm{H}), 1.93(\mathrm{~s}, 3 \mathrm{H}), 1.66-1.58(\mathrm{~m}, 1 \mathrm{H}), 1.63(\mathrm{~d}, \mathrm{~J}=6.8 \mathrm{~Hz}, 3 \mathrm{H})$.

${ }^{13} \mathrm{C}$ NMR $\left(101 \mathrm{MHz}, \mathrm{CDCl}_{3}\right): \delta 175.5,171.0,163.9,152.5,136.4,136.3,135.2,129.7,125.6,123.6$, $121.8,121.7,118.5,115.9,67.3,65.4,55.0,54.1,53.0,43.8,35.9,31.4,27.7,20.9,20.9,13.4$.

HRMS (ESI): Calculated for $\mathrm{C}_{26} \mathrm{H}_{30} \mathrm{~N}_{2} \mathrm{O}_{7}[\mathrm{M}+\mathrm{Na}]^{+}: 505.1945$, found: 505.1940 .

$[\alpha]^{25} \mathrm{D}:+213^{\circ}\left(c=0.4\right.$ in $\left.\mathrm{CHCl}_{3}\right)$.

TLC (hexane : ethyl acetate $=2: 3$ ) Rf: 0.25 (CAM, UV). 


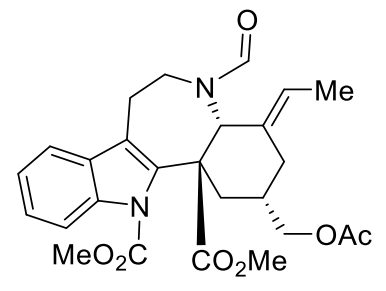

31

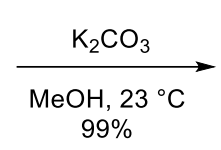

S5

\section{alcohol S5:}

To a stirred solution of $31(241.2 \mathrm{mg}, 0.4998 \mathrm{mmol}, 1$ equiv) in $\mathrm{MeOH}(20 \mathrm{~mL})$ was added $\mathrm{K}_{2} \mathrm{CO}_{3}$ (414.6 mg, 2.999 mmol, 6 equiv). After 2 hours, the resulting reaction mixture was diluted with ethyl acetate $(40 \mathrm{~mL})$ and saturated aqueous sodium chloride solution $(40 \mathrm{~mL})$ and the layers were separated. The aqueous layer was extracted with ethyl acetate $(3 \times 30 \mathrm{~mL})$, and the combined organic layers were dried over anhydrous sodium sulfate and concentrated under reduced pressure. The resulting crude residue was purified by column chromatography (silica gel: diam. $2 \mathrm{~cm}$, ht. $9 \mathrm{~cm}$; eluent : ethyl acetate) to afford $\mathbf{S} 5$ (189.2 $\mathrm{mg}, 99 \%)$ as a yellow solid.

${ }^{1} \mathrm{H}$ NMR $\left(400 \mathrm{MHz}, \mathrm{CDCl}_{3}\right): \delta 9.79(\mathrm{~s}, 1 \mathrm{H}), 8.31(\mathrm{~s}, 1 \mathrm{H}), 7.46(\mathrm{~d}, \mathrm{~J}=7.3 \mathrm{~Hz}, 1 \mathrm{H}), 7.30(\mathrm{~d}, \mathrm{~J}=8.0 \mathrm{~Hz}$, $1 \mathrm{H}), 7.15-7.10(\mathrm{~m}, 1 \mathrm{H}), 7.10-7.05(\mathrm{~m}, 1 \mathrm{H}), 5.42(\mathrm{~d}, \mathrm{~J}=6.9 \mathrm{~Hz}, 1 \mathrm{H}), 4.88(\mathrm{~s}, 1 \mathrm{H}), 4.17$ (ddd, J = 13.4, 5.3, $2.9 \mathrm{~Hz}, 1 \mathrm{H}), 3.75(\mathrm{dd}, \mathrm{J}=10.4,3.8 \mathrm{~Hz}, 1 \mathrm{H}), 3.66(\mathrm{~d}, \mathrm{~J}=3.6 \mathrm{~Hz}, 1 \mathrm{H}), 3.63(\mathrm{~d}, \mathrm{~J}=2.3 \mathrm{~Hz}, 1 \mathrm{H}), 3.59$ (s, 3H), 3.32 (ddd, J = 13.6, 11.5, 2.3 Hz, 1H), 2.98 (ddd, J = 15.3, 5.1, 2.2 Hz, 1H), 2.64 (dd, J = 15.1, $7.2 \mathrm{~Hz}, 1 \mathrm{H}), 2.42(\mathrm{dd}, \mathrm{J}=14.9,9.4 \mathrm{~Hz}, 1 \mathrm{H}), 2.32(\mathrm{dd}, \mathrm{J}=14.8,4.4 \mathrm{~Hz}, 1 \mathrm{H}), 2.13-2.03(\mathrm{~m}, 1 \mathrm{H}), 1.98$ $(\mathrm{d}, \mathrm{J}=14.1 \mathrm{~Hz}, 1 \mathrm{H}), 1.52(\mathrm{~d}, \mathrm{~J}=6.8 \mathrm{~Hz}, 3 \mathrm{H})$.

${ }^{13} \mathrm{C}$ NMR $\left(101 \mathrm{MHz}, \mathrm{CDCl}_{3}\right): \delta 174.1,165.3,135.3,133.6,132.3,128.3,123.2,121.8,119.5,118.2$, 113.6, 111.3, 66.1, 63.5, 53.2, 52.9, 39.6, 33.2, 32.0, 27.2, 23.8, 13.3 .

HRMS (ESI): Calculated for $\mathrm{C}_{22} \mathrm{H}_{26} \mathrm{~N}_{2} \mathrm{O}_{4}[\mathrm{M}+\mathrm{Na}]^{+}: 405.1785$, found: 405.1804 .

$[\alpha]^{25} \mathrm{D}:+18^{\circ}\left(\mathrm{c}=0.4\right.$ in $\left.\mathrm{CHCl}_{3}\right)$.

TLC (ethyl acetate) Rf: 0.2 (UV) 


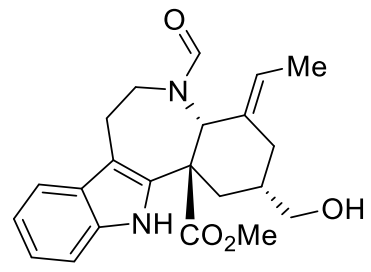

S5

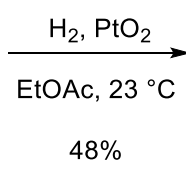

$8 \%$

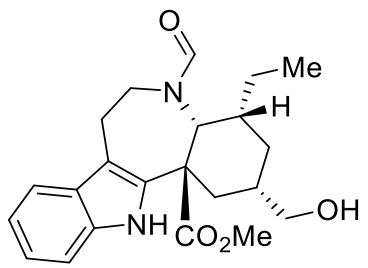

32

\section{alcohol 32:}

To a solution of $\mathbf{S} 5$ in EtOAc (10 mL) was added $\mathrm{PtO}_{2}(113.5 \mathrm{mg}, 0.4998 \mathrm{mmol}, 1$ equiv.) and was stirred under $1 \mathrm{~atm}$ of $\mathrm{H}_{2}$. After 12 hours, the $\mathrm{PtO}_{2}$ was removed by filtration through a pad of celite and the filtrate was concentrated under reduced pressure. The resulting crude residue was purified by flash column chromatography (silica gel: diam. $2 \mathrm{~cm}$, ht. $9 \mathrm{~cm}$; eluent : ethyl acetate) to afford 32 (91.3 $\mathrm{mg}, 48 \%)$ as a white solid.

${ }^{1} \mathrm{H}$ NMR $\left(400 \mathrm{MHz}, \mathrm{CDCl}_{3}\right): \delta 8.56(\mathrm{~s}, 1 \mathrm{H}), 7.49(\mathrm{~d}, \mathrm{~J}=7.0 \mathrm{~Hz}, 1 \mathrm{H}), 7.27-7.22(\mathrm{~m}, 1 \mathrm{H}), 7.17-7.08$ $(\mathrm{m}, 2 \mathrm{H}), 4.85(\mathrm{~d}, \mathrm{~J}=5.5 \mathrm{~Hz}, 1 \mathrm{H}), 4.67-4.53(\mathrm{~m}, 1 \mathrm{H}), 3.69(\mathrm{~s}, 3 \mathrm{H}), 3.60-3.50(\mathrm{~m}, 2 \mathrm{H}), 3.33(\mathrm{q}, \mathrm{J}=$ 5.2, $4.5 \mathrm{~Hz}, 1 \mathrm{H}), 3.25-3.19(\mathrm{~m}, 2 \mathrm{H}), 2.77(\mathrm{~d}, \mathrm{~J}=13.5 \mathrm{~Hz}, 1 \mathrm{H}), 1.99(\mathrm{~d}, \mathrm{~J}=12.5 \mathrm{~Hz}, 1 \mathrm{H}), 1.83(\mathrm{~s}, 2 \mathrm{H})$, $1.74-1.60(\mathrm{~m}, 2 \mathrm{H}), 1.55-1.44(\mathrm{~m}, 2 \mathrm{H}), 1.04(\mathrm{t}, \mathrm{J}=7.4 \mathrm{~Hz}, 3 \mathrm{H})$.

${ }^{13} \mathrm{C}$ NMR $\left(126 \mathrm{MHz}, \mathrm{CDCl}_{3}\right) \delta 172.5,163.4,135.2,132.1,127.7,122.3,119.6,118.2,110.7,110.2$, $67.2,56.1,54.6,53.1,45.4,38.9,36.2,32.0,30.8,25.7,20.8,11.9$.

HRMS (ESI): Calculated for $\mathrm{C}_{22} \mathrm{H}_{28} \mathrm{~N}_{2} \mathrm{O}_{4}[\mathrm{M}+\mathrm{Na}]^{+}: 407.1941$, found: 407.1953.

$[\alpha]^{25} \mathrm{D}:-47^{\circ}\left(c=0.4\right.$ in $\left.\mathrm{CHCl}_{3}\right)$.

TLC (ethyl acetate) Rf: 0.3 (UV). 
<smiles>CC[C@H]1C[C@H](CO)C[C@](COC)(c2[nH]c3ccccc3c2CCNC=O)C1</smiles>

32

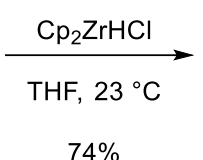

(+)-3-hydroxy-3,4-secocoronaridine (33)

\section{(+)-3-hydroxy-3,4-secocoronaridine (33):}

To a solution of $32(8.5 \mathrm{mg}, 0.02210 \mathrm{mmol}, 1$ equiv) in THF $(1 \mathrm{~mL})$ was added Schwartz reagent $(7.4 \mathrm{mg}, 0.02874 \mathrm{mmol}, 1.3$ equiv). After 1 hours, the resulting crude residue was dissolved in ethyl acetate $(10 \mathrm{~mL})$ and saturated aqueous sodium bicarbonate solution $(10 \mathrm{~mL})$ and the layers were separated. The aqueous layer was extracted with ethyl acetate $(3 \times 5 \mathrm{~mL})$, and the combined organic layers were dried over anhydrous sodium sulfate and concentrated under reduced pressure. The resulting crude residue was purified by column chromatography (silica gel: diam. $2 \mathrm{~cm}$, ht. $8 \mathrm{~cm}$; eluent : ethyl acetate) to afford $\mathbf{3 3}$ (5.8 $\mathrm{mg}, 74 \%)$ as a yellow solid.

${ }^{1} \mathrm{H}$ NMR $\left(400.1 \mathrm{MHz}, \mathrm{CDCl}_{3}\right): \delta 8.11(\mathrm{~s}, 1 \mathrm{H}), 7.46(\mathrm{~d}, \mathrm{~J}=7.8 \mathrm{~Hz}, 1 \mathrm{H}), 7.23(\mathrm{~d}, \mathrm{~J}=8.0 \mathrm{~Hz}, 1 \mathrm{H}), 7.15-$ $7.10(\mathrm{~m}, 1 \mathrm{H}), 7.08-7.03(\mathrm{~m}, 1 \mathrm{H}), 3.87(\mathrm{~d}, \mathrm{~J}=2.4 \mathrm{~Hz}, 1 \mathrm{H}), 3.71(\mathrm{~s}, 3 \mathrm{H}), 3.45(\mathrm{~d}, \mathrm{~J}=5.9 \mathrm{~Hz}, 2 \mathrm{H}), 3.41$ $-3.34(\mathrm{~m}, 1 \mathrm{H}), 3.18-3.08(\mathrm{~m}, 2 \mathrm{H}), 2.97-2.86(\mathrm{~m}, 1 \mathrm{H}), 2.51-2.43(\mathrm{~m}, 1 \mathrm{H}), 1.75(\mathrm{dtt}, \mathrm{J}=9.4,6.5$, $3.0 \mathrm{~Hz}, 1 \mathrm{H}), 1.63(\mathrm{t}, \mathrm{J}=12.9 \mathrm{~Hz}, 1 \mathrm{H}), 1.56-1.44(\mathrm{~m}, 2 \mathrm{H}), 1.43-1.34(\mathrm{~m}, 2 \mathrm{H}), 1.01-0.92(\mathrm{~m}, 1 \mathrm{H})$, $0.96(\mathrm{t}, \mathrm{J}=7.3 \mathrm{~Hz}, 3 \mathrm{H})$.

${ }^{13} \mathrm{C}$ NMR $\left(100.6 \mathrm{MHz}, \mathrm{CDCl}_{3}\right): \delta 174.4,135.4,134.4,128.7,122.2,119.5$ 118.5, 110.9 110.7, 68.0, $58.2,56.5,52.8,49.5,41.2,37.5,33.9,28.9,26.1,25.1,12.0$.

HRMS (ESI): Calculated for $\mathrm{C}_{21} \mathrm{H}_{28} \mathrm{~N}_{2} \mathrm{O}_{3}[\mathrm{M}+\mathrm{H}]^{+}: 357.2173$, found: 357.2170 .

$[\alpha]^{25} \mathrm{D}:+71^{\circ}\left(c=0.5 \text { in } \mathrm{CHCl}_{3}\right)^{6,7}$

TLC (ethyl acetate) Rf: 0.45 (UV). 


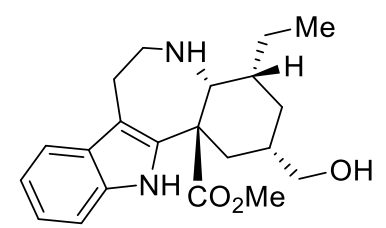

(+)-3-hydroxy-3,4-secocoronaridine (33)

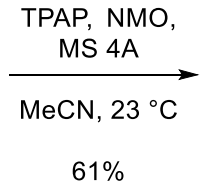

$61 \%$

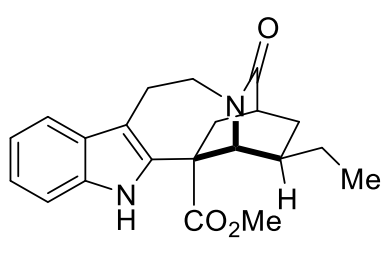

(+)-3-oxocoronaridine (35)

\section{(+)-3-oxocoronaridine (35):}

To a solution of 33 (4 mg, $0.01122 \mathrm{mmol}, 1$ equiv) in MeCN ( $1 \mathrm{~mL})$ with MS 4A was added TPAP ( $0.8 \mathrm{mg}, 0.002244 \mathrm{mmol}, 0.2$ equiv) and NMO ( $3.9 \mathrm{mg}, 0.03366 \mathrm{mmol}, 3$ equiv). After 1.5 hours, the reaction mixture was purified by column chromatography (silica gel: diam. $1 \mathrm{~cm}$, ht. $8 \mathrm{~cm}$; eluent : ethyl acetate) to afford $35(2.4 \mathrm{mg}, 61 \%)$ as a yellow oil.

${ }^{1} \mathrm{H}$ NMR $\left(400.1 \mathrm{MHz}, \mathrm{CDCl}_{3}\right): \delta 7.97(\mathrm{~s}, 1 \mathrm{H}), 7.46(\mathrm{~d}, \mathrm{~J}=7.8 \mathrm{~Hz}, 1 \mathrm{H}), 7.22(\mathrm{~d}, \mathrm{~J}=8.0 \mathrm{~Hz}, 1 \mathrm{H}), 7.13$ (ddd, J = 8.0, 7.0, 1.3 Hz, 1H), $7.07(\mathrm{td}, \mathrm{J}=7.4,1.2 \mathrm{~Hz}, 1 \mathrm{H}), 4.50(\mathrm{~s}, 1 \mathrm{H}), 4.46(\mathrm{q}, \mathrm{J}=5.4,4.4 \mathrm{~Hz}, 1 \mathrm{H})$, $3.71(\mathrm{~s}, 3 \mathrm{H}), 3.25-3.15(\mathrm{~m}, 3 \mathrm{H}), 2.68-2.56(\mathrm{~m}, 2 \mathrm{H}), 2.29$ (ddd, J = 13.3, 4.0, $2.5 \mathrm{~Hz}, 1 \mathrm{H}), 1.98$ (ddd, $\mathrm{J}=13.3,10.0,3.3 \mathrm{~Hz}, 1 \mathrm{H}), 1.78-1.69(\mathrm{~m}, 1 \mathrm{H}), 1.51(\mathrm{dq}, \mathrm{J}=14.6,7.3 \mathrm{~Hz}, 1 \mathrm{H}), 1.45-1.40(\mathrm{~m}, 1 \mathrm{H})$, $1.40-1.35(\mathrm{~m}, 1 \mathrm{H}), 0.96(\mathrm{t}, \mathrm{J}=7.4 \mathrm{~Hz}, 3 \mathrm{H})$.

${ }^{13}$ C NMR $\left(100.6 \mathrm{MHz}, \mathrm{CDCl}_{3}\right): \delta 175.9,173.3,135.9,134.1,128.0,122.6,119.8,118.6,110.8,109.6$, $56.3,55.7,53.2,42.9,38.4,36.1,35.7,31.2,27.8,21.3,11.6$.

HRMS (ESI): Calculated for $\mathrm{C}_{21} \mathrm{H}_{24} \mathrm{~N}_{2} \mathrm{O}_{3}[\mathrm{M}+\mathrm{Na}]^{+}: 375.1679$, found: 375.1686 .

$[\alpha]^{25} \mathrm{D}:+51^{\circ}\left(c=0.5\right.$ in $\left.\mathrm{CHCl}_{3}\right) .8$

TLC (ethyl acetate) Rf: 0.55 (UV). 
<smiles>CC[C@H]1C[C@@H](CO)C[C@@]2(C)c3[nH]c4ccccc4c3CCN(C=O)CC12</smiles>

32

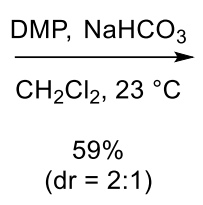

$59 \%$

$(\mathrm{dr}=2: 1)$

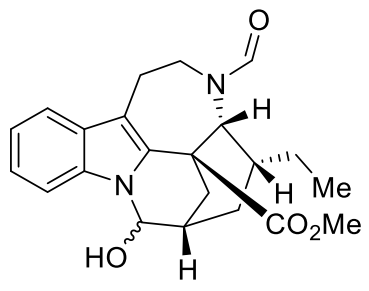

S6

\section{aminal S6:}

To a solution of 32 (52 mg, $0.1352 \mathrm{mmol}, 1$ equiv) in $\mathrm{CH}_{2} \mathrm{Cl}_{2}(6 \mathrm{~mL})$ was added Dess-Martin periodinane (114.7 $\mathrm{mg}, 0.0204 \mathrm{mmol}, 1$ equiv). After 1.5 hours, the resulting reaction mixture was diluted with $\mathrm{CH}_{2} \mathrm{Cl}_{2}(10 \mathrm{~mL})$ and saturated aqueous sodium thiosulfate solution $(10 \mathrm{~mL})$ and the layers were separated. The aqueous layer was extracted with $\mathrm{CH}_{2} \mathrm{Cl}_{2}(3 \times 10 \mathrm{~mL})$, and the combined organic layers were dried over anhydrous sodium sulfate and concentrated under reduced pressure. The resulting crude residue was purified by column chromatography (silica gel: diam. $2 \mathrm{~cm}$, ht. $9 \mathrm{~cm}$; eluent : ethyl acetate) to afford diastereomeric mixture $\mathbf{S 6}(30.5 \mathrm{mg}, 59 \%)$ as a white solid.

${ }^{1} \mathrm{H}$ NMR (major) $\left(401 \mathrm{MHz}, \mathrm{CDCl}_{3}\right): \delta 8.24(\mathrm{~s}, 1 \mathrm{H}), 7.49(\mathrm{~d}, \mathrm{~J}=2.0 \mathrm{~Hz}, 1 \mathrm{H}), 7.47(\mathrm{~d}, \mathrm{~J}=3.9 \mathrm{~Hz}, 1 \mathrm{H})$, $7.26-7.20(\mathrm{~m}, 1 \mathrm{H}), 7.17(\mathrm{td}, \mathrm{J}=7.5,1.0 \mathrm{~Hz}, 1 \mathrm{H}), 5.48(\mathrm{~s}, 1 \mathrm{H}), 4.72-4.57(\mathrm{~m}, 1 \mathrm{H}), 4.39(\mathrm{~d}, \mathrm{~J}=9.2$ $\mathrm{Hz}, 1 \mathrm{H}), 3.66(\mathrm{~s}, 3 \mathrm{H}), 2.87(\mathrm{dd}, \mathrm{J}=15.3,4.5 \mathrm{~Hz}, 1 \mathrm{H}), 2.66(\mathrm{dd}, \mathrm{J}=12.1,4.6 \mathrm{~Hz}, 1 \mathrm{H}), 2.60(\mathrm{~d}, \mathrm{~J}=9.1$ $\mathrm{Hz}, 1 \mathrm{H}), 2.53(\mathrm{dd}, \mathrm{J}=11.8,1.8 \mathrm{~Hz}, 1 \mathrm{H}), 2.50-2.43(\mathrm{~m}, 1 \mathrm{H}), 2.03(\mathrm{q}, \mathrm{J}=5.3 \mathrm{~Hz}, 1 \mathrm{H}), 1.92(\mathrm{dd}, \mathrm{J}=$ 13.0, $4.8 \mathrm{~Hz}, 1 \mathrm{H}), 1.21-1.15(\mathrm{~m}, 1 \mathrm{H}), 1.05-0.98(\mathrm{~m}, 1 \mathrm{H}), 0.88-0.85(\mathrm{~m}, 1 \mathrm{H}), 0.70(\mathrm{t}, \mathrm{J}=7.3 \mathrm{~Hz}$, $3 \mathrm{H}$ ), 0.32 (ddd, $\mathrm{J}=13.8,10.2,7.1 \mathrm{~Hz}, 1 \mathrm{H}$ ).

${ }^{13} \mathrm{C}$ NMR (major) $(101 \mathrm{MHz}): \delta 173.7,165.9,138.4,133.2,128.2,122.8,121.3,118.8,116.4,110.2$, 80.3, 61.2, 53.2, 48.8, 43.2, 38.7, 35.2, 28.4, 28.3, 23.9, 22.8, 13.5 .

${ }^{1} \mathrm{H}$ NMR (minor) $\left(401 \mathrm{MHz}, \mathrm{CDCl}_{3}\right): \delta 8.19(\mathrm{~s}, 1 \mathrm{H}), 7.50(\mathrm{~d}, \mathrm{~J}=7.0 \mathrm{~Hz}, 1 \mathrm{H}), 7.45(\mathrm{~d}, \mathrm{~J}=8.7 \mathrm{~Hz}, 1 \mathrm{H})$, $7.23-7.20(\mathrm{~m}, 1 \mathrm{H}), 7.19-7.15(\mathrm{~m}, 1 \mathrm{H}), 5.41(\mathrm{~d}, \mathrm{~J}=8.8 \mathrm{~Hz}, 1 \mathrm{H}), 3.70(\mathrm{~d}, \mathrm{~J}=2.9 \mathrm{~Hz}, 1 \mathrm{H}), 3.69(\mathrm{~s}, 3 \mathrm{H})$, $3.65-3.63(\mathrm{~m}, 1 \mathrm{H}), 3.23$ (ddd, $\mathrm{J}=13.8,10.8,2.3 \mathrm{~Hz}, 1 \mathrm{H}), 2.97-2.88(\mathrm{~m}, 1 \mathrm{H}), 2.84-2.73(\mathrm{~m}, 1 \mathrm{H})$, $2.51-2.43(\mathrm{~m}, 1 \mathrm{H}), 2.14(\mathrm{ddd}, \mathrm{J}=12.3,10.1,6.0 \mathrm{~Hz}, 1 \mathrm{H}), 2.02-1.98(\mathrm{~m}, 1 \mathrm{H}), 1.91-1.88(\mathrm{~m}, 1 \mathrm{H})$, $1.87-1.80(\mathrm{~m}, 1 \mathrm{H}), 0.99-0.90(\mathrm{~m}, 1 \mathrm{H}), 0.86-0.77(\mathrm{~m}, 1 \mathrm{H}), 0.63(\mathrm{t}, \mathrm{J}=7.3 \mathrm{~Hz}, 3 \mathrm{H}),-0.03(\mathrm{ddd}, \mathrm{J}=$ $13.6,11.0,7.0 \mathrm{~Hz}, 1 \mathrm{H})$.

${ }^{13}$ C NMR (minor) (101 MHz): $\delta$ 173.3, 164.8, 137.9, 133.8, 128.0, 122.6, 121.0, 118.4, 114.4, 110.2, $80.1,54.4,52.8,49.4,47.6,36.6,35.1,28.5,27.9,24.8,22.2,13.1$.

HRMS (ESI): Calculated for $\mathrm{C}_{22} \mathrm{H}_{26} \mathrm{~N}_{2} \mathrm{O}_{4}[\mathrm{M}+\mathrm{Na}]^{+}:$405.1785, found: 405.1806 .

TLC (ethyl acetate) Rf: 0.5 (UV). 


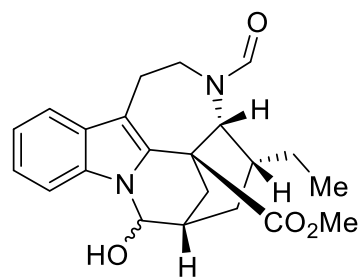

S6

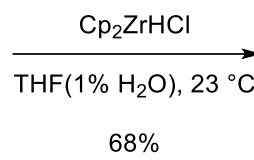

(+)-10,11-demethoxychippiine (4)

\section{(+)-10,11-demethoxychippiine (4):}

To a solution of $\mathbf{S 6}$ (12 mg, $0.03137 \mathrm{mmol}, 1$ equiv) in THF with $1 \%$ of $\mathrm{H}_{2} \mathrm{O}(1.5 \mathrm{~mL})$ was added Schwartz reagent $(12.1 \mathrm{mg}, 0.04706 \mathrm{mmol}, 1.5$ equiv). After 1 hours, Schwartz reagent $(12.1 \mathrm{mg}$, $0.04706 \mathrm{mmol}, 1.5$ equiv) was more added to the reaction mixture. After 1 hours, the resulting crude residue was dissolved in ethyl acetate $(10 \mathrm{~mL})$ and saturated aqueous sodium bicarbonate solution (10 $\mathrm{mL})$ and the layers were separated. The aqueous layer was extracted with ethyl acetate $(3 \times 10 \mathrm{~mL})$, and the combined organic layers were dried over anhydrous sodium sulfate and concentrated under reduced pressure. The resulting crude residue was purified by column chromatography (silica gel: diam. $2 \mathrm{~cm}$, ht. $11 \mathrm{~cm}$; eluent : ethyl acetate) to afford $4(7.6 \mathrm{mg}, 68 \%)$ as a yellow oil.

${ }^{1} \mathrm{H}$ NMR $\left(500 \mathrm{MHz}, \mathrm{CDCl}_{3}\right): \delta 7.48(\mathrm{~d}, \mathrm{~J}=7.7 \mathrm{~Hz}, 1 \mathrm{H}), 7.43(\mathrm{~d}, \mathrm{~J}=8.0 \mathrm{~Hz}, 1 \mathrm{H}), 7.20$ (ddd, J = 8.1, 7.1, $1.3 \mathrm{~Hz}, 1 \mathrm{H}), 7.15(\mathrm{td}, \mathrm{J}=7.5,1.1 \mathrm{~Hz}, 1 \mathrm{H}), 5.43(\mathrm{~d}, \mathrm{~J}=2.3 \mathrm{~Hz}, 1 \mathrm{H}), 3.90(\mathrm{~d}, \mathrm{~J}=7.0 \mathrm{~Hz}, 1 \mathrm{H}), 3.68(\mathrm{~s}$, $3 \mathrm{H}), 3.20-3.09(\mathrm{~m}, 1 \mathrm{H}), 2.99-2.85(\mathrm{~m}, 1 \mathrm{H}), 2.74-2.59(\mathrm{~m}, 2 \mathrm{H}), 2.52-2.41(\mathrm{~m}, 1 \mathrm{H}), 2.31(\mathrm{dd}, \mathrm{J}=$ 13.5, $1.4 \mathrm{~Hz}, 1 \mathrm{H}$ ), 2.00 (dd, J = 13.5, $5.3 \mathrm{~Hz}, 1 \mathrm{H}$ ), 1.81 (dqd, J = 14.2, 7.1, 3.4 Hz, 1H), 1.60 (ddd, J = $14.1,10.4,3.4 \mathrm{~Hz}, 1 \mathrm{H}), 1.41-1.30(\mathrm{~m}, 1 \mathrm{H}), 0.97(\mathrm{tt}, \mathrm{J}=13.1,6.0 \mathrm{~Hz}, 1 \mathrm{H}), 0.89(\mathrm{t}, \mathrm{J}=7.2 \mathrm{~Hz}, 3 \mathrm{H})$, $0.30(\mathrm{td}, \mathrm{J}=14.2,7.0 \mathrm{~Hz}, 1 \mathrm{H})$.

${ }^{13} \mathrm{C} \mathrm{NMR}\left(126 \mathrm{MHz}, \mathrm{CDCl}_{3}\right): \delta 176.4,137.4,131.7,129.2,121.9,120.6,118.5,109.8,109.5,79.7,58.5$, 52.9, 49.4, 40.6, 36.1, 34.6, 24.4, 24.2, 24.1, 23.2, 12.6.

HRMS (ESI): Calculated for $\mathrm{C}_{21} \mathrm{H}_{26} \mathrm{~N}_{2} \mathrm{O}_{3}[\mathrm{M}+\mathrm{H}]^{+}: 355.2016$, found: 355.2013 .

$[\alpha]^{25} \mathrm{D}:+27^{\circ}\left(c=0.2\right.$ in $\left.\mathrm{CHCl}_{3}\right) .{ }^{9,10}$

TLC (ethyl acetate) Rf: 0.3 (UV). 


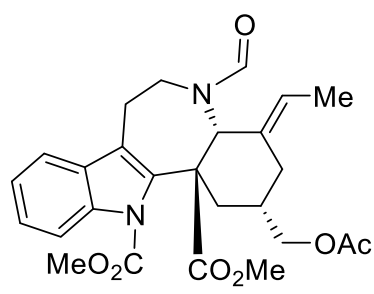

31

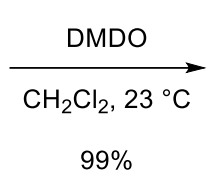

$9 \%$

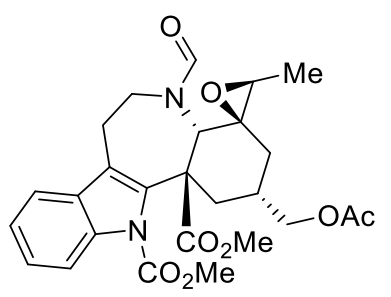

S7

\section{epoxide S7:}

To a stirred solution of 31 (550 mg, $1.139 \mathrm{mmol}, 1$ equiv) in $\mathrm{CH}_{2} \mathrm{Cl}_{2}(20 \mathrm{~mL})$ was added freshly prepared dimethyldioxirane in acetone $(31.5 \mathrm{~mL}, 65 \mathrm{mM}, 2.051 \mathrm{mmol}, 1.8$ equiv). After 1.5 hours, the reaction mixture was concentrated under reduced pressure to afforded $\mathbf{S 7}(562.5 \mathrm{mg}, 99 \%)$ as a yellow solid.

${ }^{1} \mathrm{H}$ NMR $\left(600 \mathrm{MHz}, \mathrm{CDCl}_{3}\right): \delta 8.01(\mathrm{~d}, \mathrm{~J}=8.2 \mathrm{~Hz}, 1 \mathrm{H}), 7.83(\mathrm{~s}, 1 \mathrm{H}), 7.47(\mathrm{~d}, \mathrm{~J}=7.6 \mathrm{~Hz}, 1 \mathrm{H}), 7.35(\mathrm{t}, \mathrm{J}$ $=7.5 \mathrm{~Hz}, 1 \mathrm{H}), 7.31(\mathrm{t}, \mathrm{J}=7.4 \mathrm{~Hz}, 1 \mathrm{H}), 4.19(\mathrm{~s}, 1 \mathrm{H}), 3.98(\mathrm{~s}, 3 \mathrm{H}), 3.92(\mathrm{~d}, \mathrm{~J}=13.9 \mathrm{~Hz}, 1 \mathrm{H}), 3.68(\mathrm{~d}, \mathrm{~J}=$ $5.7 \mathrm{~Hz}, 2 \mathrm{H}), 3.66-3.58(\mathrm{~m}, 2 \mathrm{H}), 3.55(\mathrm{~s}, 3 \mathrm{H}), 3.23-3.16(\mathrm{~m}, 2 \mathrm{H}), 2.96-2.88(\mathrm{~m}, 2 \mathrm{H}), 2.47(\mathrm{ddt}, \mathrm{J}=$ 16.5, 11.3, $5.7 \mathrm{~Hz}, 1 \mathrm{H}), 2.20$ (dd, J = 15.7, $6.5 \mathrm{~Hz}, 1 \mathrm{H}), 1.87(\mathrm{~s}, 3 \mathrm{H}), 1.59(\mathrm{t}, \mathrm{J}=13.5 \mathrm{~Hz}, 1 \mathrm{H}), 1.31$ (d, $\mathrm{J}=5.6 \mathrm{~Hz}, 3 \mathrm{H})$.

${ }^{13} \mathrm{C}$ NMR $\left(151 \mathrm{MHz}, \mathrm{CDCl}_{3}\right): \delta 174.6,171.1,163.4,152.5,136.3,135.9,129.3,125.9,123.7,121.1$, 118.4, 116.1, 67.9, 66.1, 64.1, 59.4, 54.6, 54.3, 53.1, 43.8, 33.7, 28.7, 27.8, 20.9, 19.7, 13.7.

HRMS (ESI): Calculated for $\mathrm{C}_{26} \mathrm{H}_{30} \mathrm{~N}_{2} \mathrm{O}_{8}[\mathrm{M}+\mathrm{Na}]^{+}: 521.1894$, found: 521.1912.

$[\alpha]^{25} \mathrm{D}:+180^{\circ}\left(c=0.4\right.$ in $\left.\mathrm{CHCl}_{3}\right)$.

TLC (hexane : ethyl acetate $=2: 3$ ) Rf: 0.22 (UV). 


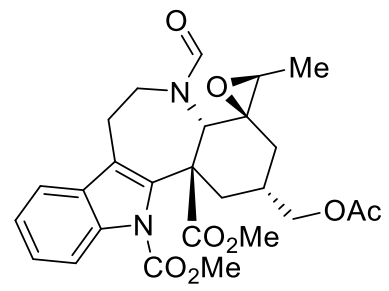

s7

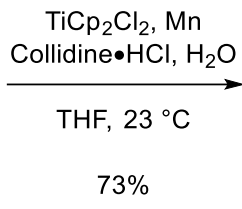

alcohol 36:

To a solution of collidine $\cdot \mathrm{HCl}(569 \mathrm{mg}, 3.610 \mathrm{mmol}, 2.5$ equiv) in THF (12 mL) was added $\mathrm{TiCp}_{2} \mathrm{Cl}_{2}$ (599.2 mg, $2.876 \mathrm{mmol}, 2.5$ equiv) and Mn dust (264 mg, $2.876 \mathrm{mmol}, 2.5$ equiv). After 1 hours, to this reaction mixture was added to a solution of $\mathbf{S 7}(115.4 \mathrm{mg}, 0.4011 \mathrm{mmol}, 1$ equiv) in THF with $1 \% \mathrm{H}_{2} \mathrm{O}(12 \mathrm{~mL})$. After 1.5 hours, the reaction mixture was diluted with ethyl acetate $(20 \mathrm{~mL})$ and $2 \mathrm{M}$ $\mathrm{HCl}$ solution $(20 \mathrm{~mL})$ and the layers were separated. The aqueous layer was extracted with ethyl acetate $(3 \times 10 \mathrm{~mL})$, and the combined organic layers were dried over anhydrous sodium sulfate and concentrated under reduced pressure. The resulting crude residue was purified by flash column chromatography (silica gel: diam. $3 \mathrm{~cm}$, ht. $10 \mathrm{~cm}$; eluent : ethyl acetate : $\mathrm{NH}_{4} \mathrm{OH}=100: 1$ ) to afford $36(146.5 \mathrm{mg}, 73 \%)$ as a yellow solid.

${ }^{1} \mathrm{H}$ NMR $\left(400 \mathrm{MHz}, \mathrm{CDCl}_{3}\right): \delta 8.00(\mathrm{~s}, 1 \mathrm{H}), 7.96(\mathrm{~d}, \mathrm{~J}=8.3 \mathrm{~Hz}, 1 \mathrm{H}), 7.45(\mathrm{~d}, \mathrm{~J}=7.5 \mathrm{~Hz}, 1 \mathrm{H}), 7.36-$ $7.30(\mathrm{~m}, 1 \mathrm{H}), 7.30-7.25(\mathrm{~m}, 1 \mathrm{H}), 4.08-4.01(\mathrm{~m}, 2 \mathrm{H}), 3.99(\mathrm{~m}, 1 \mathrm{H}), 3.95(\mathrm{~s}, 3 \mathrm{H}), 3.62(\mathrm{dd}, \mathrm{J}=15.1$, $3.5 \mathrm{~Hz}, 1 \mathrm{H}), 3.56(\mathrm{~s}, 3 \mathrm{H}), 3.39$ (dd, J = 10.7, $7.2 \mathrm{~Hz}, 1 \mathrm{H}), 3.18(\mathrm{~d}, \mathrm{~J}=18.5 \mathrm{~Hz}, 1 \mathrm{H}), 2.97-2.89$ (m, $1 \mathrm{H}), 2.89-2.81(\mathrm{~m}, 1 \mathrm{H}), 2.62(\mathrm{dd}, \mathrm{J}=15.7,5.1 \mathrm{~Hz}, 1 \mathrm{H}), 2.54(\mathrm{t}, \mathrm{J}=13.0 \mathrm{~Hz}, 1 \mathrm{H}), 2.24(\mathrm{~m}, 1 \mathrm{H}), 1.94$ $(\mathrm{s}, 3 \mathrm{H}), 1.93(\mathrm{~m}, 1 \mathrm{H}), 1.75(\mathrm{td}, \mathrm{J}=12.8,12.3,5.2 \mathrm{~Hz}, 1 \mathrm{H}), 1.67-1.58(\mathrm{~m}, 1 \mathrm{H}), 1.15(\mathrm{~d}, \mathrm{~J}=6.5 \mathrm{~Hz}, 3 \mathrm{H})$.

${ }^{13} \mathrm{C}$ NMR $\left(101 \mathrm{MHz}_{\mathrm{CDCl}}\right): \delta 174.7,170.8,164.8,152.4,136.3,135.3,129.8,125.9,123.7,121.8$, $118.4,115.5,66.9,65.4,65.3,55.0,54.1,52.8,43.4,40.3,34.6,31.2,25.0,22.0,21.5,20.9$.

HRMS (ESI): Calculated for $\mathrm{C}_{26} \mathrm{H}_{32} \mathrm{~N}_{2} \mathrm{O}_{8}[\mathrm{M}+\mathrm{Na}]^{+}: 523.2051$, found: 523.2043.

$[\alpha]^{25} \mathrm{D}:+168^{\circ}\left(c=0.4\right.$ in $\left.\mathrm{CHCl}_{3}\right)$.

TLC (ethyl acetate) Rf: 0.2 (UV). 


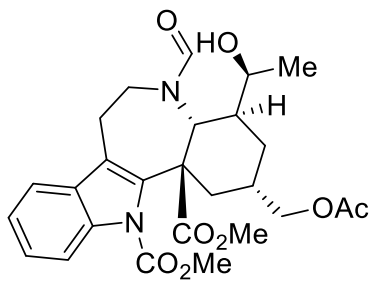

36

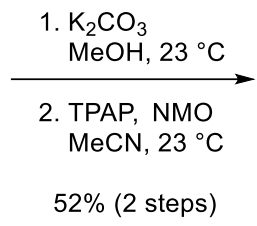

$52 \%$ (2 steps)

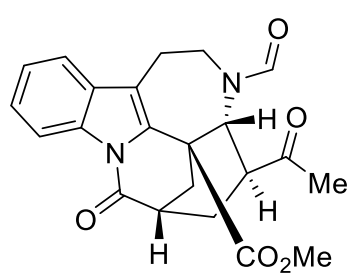

37

\section{lactam 37:}

To a solution of 36 ( $114.5 \mathrm{mg}, 0.2287 \mathrm{mmol}, 1$ equiv) in $\mathrm{MeOH}(6 \mathrm{~mL})$ was added $\mathrm{K}_{2} \mathrm{CO}_{3}(189.7$ $\mathrm{mg}, 1.3725 \mathrm{mmol}, 6$ equiv). After 1 hours, the resulting reaction mixture was diluted with ethyl acetate $(10 \mathrm{~mL})$ and saturated aqueous sodium chloride solution $(10 \mathrm{~mL})$ and the layers were separated. The aqueous layer was extracted with ethyl acetate $(3 \times 10 \mathrm{~mL})$, and the combined organic layers were dried over anhydrous sodium sulfate and concentrated under reduced pressure.

To a solution of the resulting crude residue in MeCN (6 mL) was added TPAP (16.1 mg, 0.0458 mmol, 0.2 equiv) and NMO (134.0 mg, $1.1437 \mathrm{mmol}, 5$ equiv). After 1.5 hours, the reaction mixture was purified by column chromatography (silica gel: diam. $2 \mathrm{~cm}$, ht. $10 \mathrm{~cm}$; eluent : ethyl acetate) to afford $37(47 \mathrm{mg}, 52 \%)$ as a yellow solid.

${ }^{1} \mathrm{H}$ NMR $\left(400 \mathrm{MHz}, \mathrm{CDCl}_{3}\right): \delta 8.36(\mathrm{~d}, \mathrm{~J}=7.7 \mathrm{~Hz}, 1 \mathrm{H}), 8.19(\mathrm{~s}, 1 \mathrm{H}), 7.52(\mathrm{~d}, \mathrm{~J}=7.4 \mathrm{~Hz}, 1 \mathrm{H}), 7.44-$ $7.34(\mathrm{~m}, 2 \mathrm{H}), 4.60(\mathrm{dt}, \mathrm{J}=14.2,3.0 \mathrm{~Hz}, 1 \mathrm{H}), 4.37(\mathrm{~d}, \mathrm{~J}=11.1 \mathrm{~Hz}, 1 \mathrm{H}), 3.78(\mathrm{~s}, 3 \mathrm{H}), 3.13(\mathrm{~d}, \mathrm{~J}=2.9 \mathrm{~Hz}$, $1 \mathrm{H}), 2.87(\mathrm{dd}, \mathrm{J}=15.9,4.2 \mathrm{~Hz}, 1 \mathrm{H}), 2.63(\mathrm{t}, \mathrm{J}=12.2 \mathrm{~Hz}, 3 \mathrm{H}), 2.40(\mathrm{t}, \mathrm{J}=13.1 \mathrm{~Hz}, 1 \mathrm{H}), 2.29-2.24(\mathrm{~m}$, 2H), $1.96(\mathrm{~s}, 3 \mathrm{H}), 1.85(\mathrm{td}, \mathrm{J}=13.6,5.3 \mathrm{~Hz}, 1 \mathrm{H})$.

${ }^{13} \mathrm{C}$ NMR $\left(101 \mathrm{MHz}, \mathrm{CDCl}_{3}\right): \delta 207.0,171.4,169.5,165.2,135.3,132.9,130.6,125.6,125.0,121.3$, $119.0,117.5,60.3,53.5,49.4,45.9,39.1,37.9,34.0,31.5,30.4,24.0$.

HRMS (ESI): Calculated for $\mathrm{C}_{22} \mathrm{H}_{22} \mathrm{~N}_{2} \mathrm{O}_{5}[\mathrm{M}+\mathrm{Na}]^{+}: 417.1421$, found: 417.1440 .

$[\alpha]^{25} \mathrm{D}:+152^{\circ}\left(c=0.5\right.$ in $\left.\mathrm{CHCl}_{3}\right)$.

TLC (ethyl acetate) Rf: 0.45 (UV). 


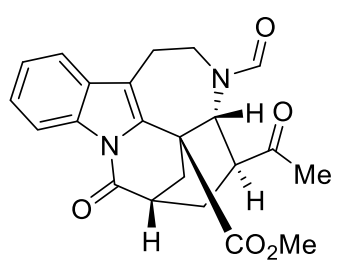

37

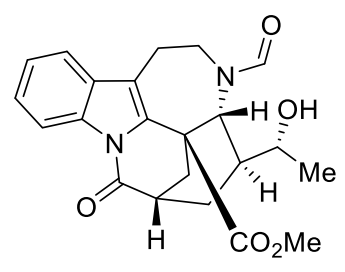

58
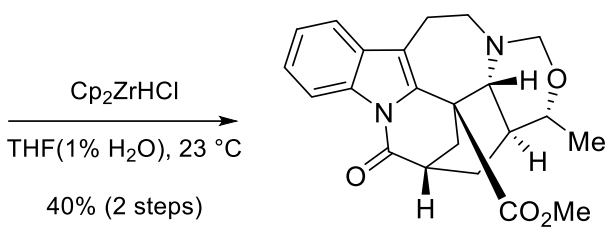

39

\section{hexacycle 39:}

To a stirred solution of $37(8.9 \mathrm{mg}, 0.0177 \mathrm{mmol}$, 1 equiv) in THF (1 mL) was added (1.57 mL, 2 equiv, THF solution, $1 \mathrm{M}$ ) at $-78^{\circ} \mathrm{C}$. After $40 \mathrm{~min}$, the reaction mixture was slowly warm up to $23^{\circ} \mathrm{C}$ and the reaction mixture was diluted with ethyl acetate $(10 \mathrm{~mL})$ and saturated aqueous ammonium chloride solution $(10 \mathrm{~mL})$ and the layers were separated. The aqueous layer was extracted with ethyl acetate $(3 \times 10 \mathrm{~mL})$, and the combined organic layers were dried over anhydrous sodium sulfate and concentrated under reduced pressure. The Intermediate $\mathbf{S 8}$ was immediately moved to the next step without further purification.

To a solution of $\mathbf{S 8}\left(12.3 \mathrm{mg}, 0.0418 \mathrm{mmol}, 1\right.$ equiv) in THF with $1 \%$ of $\mathrm{H}_{2} \mathrm{O}(1 \mathrm{~mL})$ was added Schwartz reagent $(4.8 \mathrm{mg}, 0.0627 \mathrm{mmol}, 1.5$ equiv). After 1 hours, Schwartz reagent $(4.8 \mathrm{mg}, 0.0627$ mmol, 1.5 equiv) was more added to the reaction mixture. After 1 hours, the resulting crude residue was dissolved in ethyl acetate $(10 \mathrm{~mL})$ and saturated aqueous sodium bicarbonate solution $(10 \mathrm{~mL})$ and the layers were separated. The aqueous layer was extracted with ethyl acetate $(3 \times 10 \mathrm{~mL})$, and the combined organic layers were dried over anhydrous sodium sulfate and concentrated under reduced pressure. The resulting crude residue was purified by column chromatography (silica gel: diam. $1 \mathrm{~cm}$, ht. $8 \mathrm{~cm}$; eluent : ethyl acetate) to afford $39(5.2 \mathrm{mg}, 40 \%)$ as a yellow solid.

${ }^{1} \mathrm{H}$ NMR $\left(400.1 \mathrm{MHz}, \mathrm{CDCl}_{3}\right): \delta 8.32(\mathrm{dd}, \mathrm{J}=5.0,1.9 \mathrm{~Hz}, 1 \mathrm{H}), 7.51(\mathrm{dd}, \mathrm{J}=4.4,1.4 \mathrm{~Hz}, 1 \mathrm{H}), 7.36-$ $7.33(\mathrm{~m}, 2 \mathrm{H}), 4.64(\mathrm{~d}, \mathrm{~J}=10.6 \mathrm{~Hz}, 1 \mathrm{H}), 4.43(\mathrm{~d}, \mathrm{~J}=10.6 \mathrm{~Hz}, 1 \mathrm{H}), 3.79(\mathrm{~s}, 3 \mathrm{H}), 3.56(\mathrm{~d}, \mathrm{~J}=11.1 \mathrm{~Hz}$, $1 \mathrm{H}), 3.38-3.29(\mathrm{~m}, 1 \mathrm{H}), 3.03(\mathrm{dd}, \mathrm{J}=5.8,3.2 \mathrm{~Hz}, 1 \mathrm{H}), 2.97(\mathrm{~d}, \mathrm{~J}=12.9 \mathrm{~Hz}, 1 \mathrm{H}), 2.92-2.80(\mathrm{~m}, 2 \mathrm{H})$, $2.80-2.74(\mathrm{~m}, 1 \mathrm{H}), 2.57(\mathrm{dt}, \mathrm{J}=13.1,2.8 \mathrm{~Hz}, 1 \mathrm{H}), 2.18(\mathrm{dd}, \mathrm{J}=13.1,2.8 \mathrm{~Hz}, 1 \mathrm{H}), 2.00(\mathrm{dd}, \mathrm{J}=12.5$, $4.0 \mathrm{~Hz}, 1 \mathrm{H}), 1.43-1.32(\mathrm{~m}, 1 \mathrm{H}), 1.19-1.05(\mathrm{~m}, 1 \mathrm{H}), 0.96(\mathrm{~d}, \mathrm{~J}=6.1 \mathrm{~Hz}, 3 \mathrm{H})$.

${ }^{13} \mathrm{C}$ NMR $\left(101 \mathrm{MHz}, \mathrm{CDCl}_{3}\right): \delta 172.6,170.6,135.5,133.1,130.9,124.9,124.6,120.8,118.7,117.4$, 89.1, 79.0, 63.9, 53.1, 47.7, 47.3, 39.6, 35.3, 33.9, 30.5, 23.3, 18.9.

HRMS (ESI): Calculated for $\mathrm{C}_{22} \mathrm{H}_{24} \mathrm{~N}_{2} \mathrm{O}_{4}[\mathrm{M}+\mathrm{H}]+: 381.1809$, found: 381.1809 .

$[\alpha]^{25}$ : $+141^{\circ}\left(c=0.3\right.$ in $\left.\mathrm{CHCl}_{3}\right)$.

TLC (ethyl acetate) Rf: 0.4 (UV). 


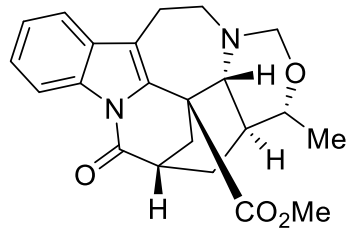

39

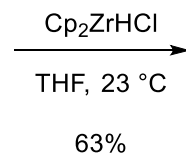

$63 \%$

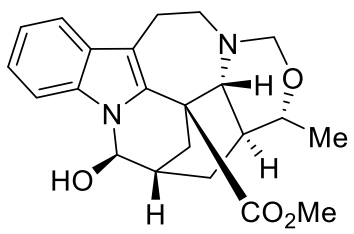

(-)-dippinine C (5)

\section{(-)-dippinine C (5):}

To a solution of 39 ( $3.0 \mathrm{mg}, 0.0788 \mathrm{mmol}, 1$ equiv) in THF $(1 \mathrm{~mL})$ was added Schwartz reagent (4.8 mg, $0.0627 \mathrm{mmol}, 4.5$ equiv). After 1.5 hours, the resulting crude residue was dissolved in ethyl acetate $(10 \mathrm{~mL})$ and saturated aqueous sodium bicarbonate solution $(10 \mathrm{~mL})$ and the layers were separated. The aqueous layer was extracted with ethyl acetate $(3 \times 10 \mathrm{~mL})$, and the combined organic layers were dried over anhydrous sodium sulfate and concentrated under reduced pressure. The resulting crude residue was purified by column chromatography (silica gel: diam. $1 \mathrm{~cm}$, ht. $8 \mathrm{~cm}$; eluent : hexane : ethyl acetate $=3: 1)$ to afford $5(1.9 \mathrm{mg}, 63 \%)$ as a yellow oil.

${ }^{1} \mathrm{H}$ NMR $\left(400.1 \mathrm{MHz}, \mathrm{CDCl}_{3}\right): \delta 7.54(\mathrm{~d}, \mathrm{~J}=6.1 \mathrm{~Hz}, 1 \mathrm{H}), 7.49(\mathrm{~d}, \mathrm{~J}=7.0 \mathrm{~Hz}, 1 \mathrm{H}), 7.23(\mathrm{~d}, \mathrm{~J}=1.8 \mathrm{~Hz}$, $1 \mathrm{H}), 7.21(\mathrm{t}, \mathrm{J}=1.9 \mathrm{~Hz}, 1 \mathrm{H}), 5.57(\mathrm{~s}, 1 \mathrm{H}), 4.61(\mathrm{~d}, \mathrm{~J}=10.5 \mathrm{~Hz}, 1 \mathrm{H}), 4.40(\mathrm{~d}, \mathrm{~J}=10.5 \mathrm{~Hz}, 1 \mathrm{H}), 3.79(\mathrm{~s}$, $3 \mathrm{H}), 3.43(\mathrm{~d}, \mathrm{~J}=11.0 \mathrm{~Hz}, 1 \mathrm{H}), 3.25(\mathrm{dd}, \mathrm{J}=9.0,6.1 \mathrm{~Hz}, 1 \mathrm{H}), 2.94(\mathrm{~d}, \mathrm{~J}=5.0 \mathrm{~Hz}, 1 \mathrm{H}), 2.82-2.77(\mathrm{~m}$, $2 \mathrm{H}), 2.76-2.72(\mathrm{~m}, 1 \mathrm{H}), 2.66(\mathrm{dt}, \mathrm{J}=13.3,2.4 \mathrm{~Hz}, 1 \mathrm{H}), 2.47-2.43(\mathrm{~m}, 1 \mathrm{H}), 2.03(\mathrm{~d}, \mathrm{~J}=3.7 \mathrm{~Hz}, 1 \mathrm{H})$, $1.66(\mathrm{dd}, \mathrm{J}=14.4,5.4 \mathrm{~Hz}, 1 \mathrm{H}), 1.26-1.24(\mathrm{~m}, 1 \mathrm{H}), 0.91(\mathrm{~d}, \mathrm{~J}=6.2 \mathrm{~Hz}, 3 \mathrm{H}), 0.72-0.60(\mathrm{~m}, 1 \mathrm{H})$.

${ }^{13} \mathrm{C} \mathrm{NMR}\left(101 \mathrm{MHz}, \mathrm{CDCl}_{3}\right): \delta 173.7,137.0,132.8,128.4,122.0,121.1,119.0,114.2,110.0,88.9,79.7$, 79.6, 64.8, 52.9, 47.9, 47.8, 36.1, 33.3, 31.5, 29.2, 23.4, 18.8 .

HRMS (ESI): Calculated for $\mathrm{C}_{22} \mathrm{H}_{26} \mathrm{~N}_{2} \mathrm{O}_{4}[\mathrm{M}+\mathrm{H}]+$ : 383.1965, found: 383.1962.

$[\alpha]^{25} \mathrm{D}:-25^{\circ}\left(\mathrm{C}=0.25\right.$ in $\left.\mathrm{CHCl}_{3}\right){ }^{11}$

TLC (ethyl acetate) Rf: 0.4 (UV). 


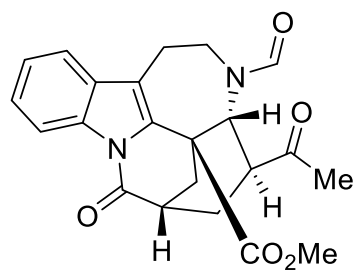

37

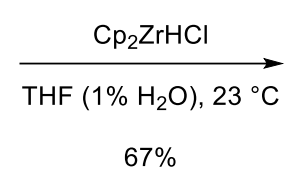

$67 \%$

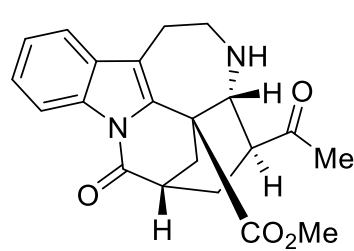

40

\section{amine 40:}

To a solution of 37 (10.5 mg, $0.02662 \mathrm{mmol}, 1$ equiv) in THF with $1 \%$ of $\mathrm{H}_{2} \mathrm{O}(2 \mathrm{~mL})$ was added Schwartz reagent (10.3 mg, $0.03993 \mathrm{mmol}, 1.5$ equiv). After 1 hours, Schwartz reagent $(10.3 \mathrm{mg}$, $0.03993 \mathrm{mmol}, 1.5$ equiv) was more added to the reaction mixture. After 1 hours, the resulting crude residue was dissolved in ethyl acetate $(10 \mathrm{~mL})$ and saturated aqueous sodium bicarbonate solution $(10$ $\mathrm{mL})$ and the layers were separated. The aqueous layer was extracted with ethyl acetate $(3 \times 10 \mathrm{~mL})$, and the combined organic layers were dried over anhydrous sodium sulfate and concentrated under reduced pressure. The resulting crude residue was purified by column chromatography (silica gel: diam. $2 \mathrm{~cm}$, ht. $8 \mathrm{~cm}$; eluent : hexane : ethyl acetate $=3: 1)$ to afford $40(6.5 \mathrm{mg}, 67 \%)$ as a yellow solid.

${ }^{1} \mathrm{H}$ NMR $\left(400 \mathrm{MHz}, \mathrm{CDCl}_{3}\right)$ : $\delta 8.33(\mathrm{dd}, \mathrm{J}=7.1,1.5 \mathrm{~Hz}, 1 \mathrm{H}), 7.45$ (dd, J = 7.3, $\left.2.3 \mathrm{~Hz}, 1 \mathrm{H}\right), 7.38-7.31$ $(\mathrm{m}, 2 \mathrm{H}), 3.81(\mathrm{~s}, 3 \mathrm{H}), 3.60(\mathrm{~d}, \mathrm{~J}=11.5 \mathrm{~Hz}, 1 \mathrm{H}), 3.05(\mathrm{dd}, \mathrm{J}=5.4,2.7 \mathrm{~Hz}, 1 \mathrm{H}), 2.94-2.83(\mathrm{~m}, 1 \mathrm{H})$, $2.77-2.60(\mathrm{~m}, 3 \mathrm{H}), 2.59-2.49(\mathrm{~m}, 2 \mathrm{H}), 2.15(\mathrm{dd}, \mathrm{J}=13.1,3.0 \mathrm{~Hz}, 1 \mathrm{H}), 2.12-2.07(\mathrm{~m}, 1 \mathrm{H}), 2.07(\mathrm{~s}$, $3 \mathrm{H}), 1.94-1.84(\mathrm{~m}, 1 \mathrm{H})$.

${ }^{13} \mathrm{C}$ NMR $\left(101 \mathrm{MHz}, \mathrm{CDCl}_{3}\right): \delta 209.8,173.3,170.5,135.3,134.4,131.1,125.0,124.6,121.3,118.8$, $117.4,57.3,53.1,51.2,46.5,41.0,39.4,33.9,31.4,30.1,26.6$.

HRMS (ESI): Calculated for $\mathrm{C}_{21} \mathrm{H}_{22} \mathrm{~N}_{2} \mathrm{O}_{4}[\mathrm{M}+\mathrm{H}]+: 367.1652$, found: 367.1661

$[\alpha]^{25} \mathrm{D}:+133^{\circ}\left(c=0.3\right.$ in $\left.\mathrm{CHCl}_{3}\right)$.

TLC (ethyl acetate) Rf: 0.5 (UV). 
<smiles>COC(=O)[C@H]1[C@@H]2NCCc3c4n(c5ccccc35)C(=O)[C@@H]1[C@@H]2N[C@H]4C(C)=O</smiles>

40

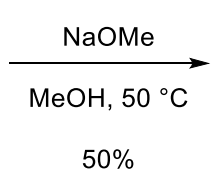

$50 \%$

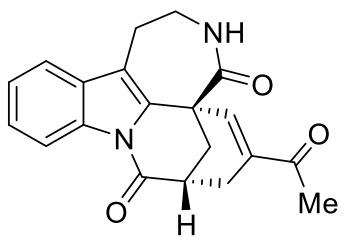

42

\section{bislactam 42:}

To a solution of 40 (13.3 mg, $0.03629 \mathrm{mmol}, 1$ equiv) in $\mathrm{MeOH}(1.5 \mathrm{~mL})$ was added $\mathrm{NaOMe}$ $\left(78 \mu \mathrm{L}, 0.3629 \mathrm{mmol}, 10\right.$ equiv, $\mathrm{MeOH}$ solution, $25 \%$ ) and the temperature was raised to $50^{\circ} \mathrm{C}$. After 4 hours, the reaction mixture was cooled to $23^{\circ} \mathrm{C}$ and was dissolved in ethyl acetate $(10 \mathrm{~mL})$ and saturated aqueous sodium bicarbonate solution $(10 \mathrm{~mL})$ and the layers were separated. The aqueous layer was extracted with ethyl acetate $(3 \times 10 \mathrm{~mL})$, and the combined organic layers were dried over anhydrous sodium sulfate and concentrated under reduced pressure. The resulting crude residue was purified by column chromatography (silica gel: diam. $2 \mathrm{~cm}$, ht. $8 \mathrm{~cm}$; eluent : ethyl acetate) to afford 42 $(5.2 \mathrm{mg}, 40 \%)$ as a yellow solid.

${ }^{1} \mathrm{H}$ NMR $\left(400.1 \mathrm{MHz}, \mathrm{CDCl}_{3}\right): \delta 8.46(\mathrm{~d}, \mathrm{~J}=8.1 \mathrm{~Hz}, 1 \mathrm{H}), 7.40(\mathrm{~d}, \mathrm{~J}=7.7 \mathrm{~Hz}, 1 \mathrm{H}), 7.35(\mathrm{t}, \mathrm{J}=7.6 \mathrm{~Hz}$, $1 \mathrm{H}), 7.29(\mathrm{t}, \mathrm{J}=7.4 \mathrm{~Hz}, 1 \mathrm{H}), 7.03(\mathrm{~s}, 1 \mathrm{H}), 6.48(\mathrm{t}, \mathrm{J}=6.8 \mathrm{~Hz}, 1 \mathrm{H}), 3.90(\mathrm{ddt}, \mathrm{J}=15.1,10.3,5.2 \mathrm{~Hz}, 1 \mathrm{H})$, 3.64 (ddd, $\mathrm{J}=13.9,7.4,3.1 \mathrm{~Hz}, 1 \mathrm{H}), 3.40(\mathrm{dd}, \mathrm{J}=5.8,2.6 \mathrm{~Hz}, 1 \mathrm{H}), 3.03-2.94(\mathrm{~m}, 2 \mathrm{H}), 2.76(\mathrm{~d}, \mathrm{~J}=$ $7.2 \mathrm{~Hz}, 2 \mathrm{H}$ ), 2.56 (dd, J = 13.8, $2.5 \mathrm{~Hz}, 1 \mathrm{H}), 2.46(\mathrm{dd}, \mathrm{J}=13.4,3.8 \mathrm{~Hz}, 1 \mathrm{H}), 2.25(\mathrm{~s}, 3 \mathrm{H})$.

${ }^{13} \mathrm{C}$ NMR $\left(100.6 \mathrm{MHz}, \mathrm{CDCl}_{3}\right): \delta$ 197.8, 172.7, 171.6, 137.9, 137.2, 134.9, 130.8, 129.7, 126.2, 124.6, 118.3, 118.0, 116.9, 44.3, 41.2, 36.9, 30.9, 27.3, 25.9, 25.7.

HRMS (ESI): Calculated for $\mathrm{C}_{20} \mathrm{H}_{18} \mathrm{~N}_{2} \mathrm{O}_{3}[\mathrm{M}+\mathrm{Na}]^{+}: 357.1210$, found: 357.1216 .

$[\alpha]^{25} \mathrm{D}:-67^{\circ}\left(c=0.2\right.$ in $\left.\mathrm{CHCl}_{3}\right)$.

TLC (ethyl acetate) Rf: 0.4 (UV). 
<smiles>C=C(C)C(=O)C[C@]12CCC(=O)NCCc3c1n(c1ccccc31)C2=O</smiles>

42

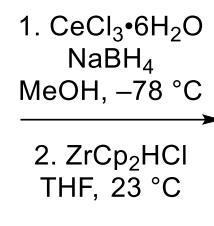

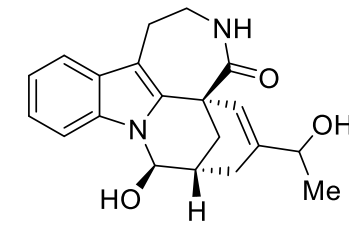

s9

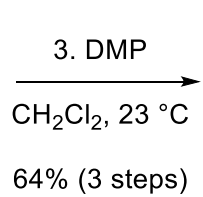

(-)-tronocarpine (6)

\section{(-)-tronocarpine (6):}

To a solution of $42\left(7 \mathrm{mg}, 0.02093 \mathrm{mmol}, 1\right.$ equiv) in $\mathrm{MeOH}(1.5 \mathrm{~mL})$ was added $\mathrm{CeCl}_{3} \cdot 6 \mathrm{H}_{2} \mathrm{O}$ (11.7 mg, $0.03140 \mathrm{mmol}, 1.5$ equiv). After $40 \mathrm{~min}, \mathrm{NaBH}_{4}(1.2 \mathrm{mg}, 0.03140 \mathrm{mmol}, 1.5$ equiv) was added to mixed solution at $-78^{\circ} \mathrm{C}$. After 1.5 hours, the resulting crude residue was dissolved in ethyl acetate $(10 \mathrm{~mL})$ and saturated aqueous sodium bicarbonate solution $(10 \mathrm{~mL})$ and the layers were separated. The aqueous layer was extracted with ethyl acetate $(3 \times 10 \mathrm{~mL})$, and the combined organic layers were dried over anhydrous sodium sulfate and concentrated under reduced pressure. The resulting alcohol intermediate was used in the next step without further purification.

To a solution of alcohol in THF ( $1 \mathrm{~mL})$ was added Schwartz reagent $(27 \mathrm{mg}, 0.1046 \mathrm{mmol}, 5$ equiv). After 1.5 hours, the resulting crude residue was dissolved in ethyl acetate $(10 \mathrm{~mL})$ and saturated aqueous sodium bicarbonate solution $(10 \mathrm{~mL})$ and the layers were separated. The aqueous layer was extracted with ethyl acetate $(3 \times 10 \mathrm{~mL})$, and the combined organic layers were dried over anhydrous sodium sulfate and concentrated under reduced pressure. The resulting alcohol intermediate $\mathbf{S 9}$ was used in the next step without further purification.

To a solution of $\mathbf{S 9}$ in $\mathrm{CH}_{2} \mathrm{Cl}_{2}(1 \mathrm{~mL})$ was added Dess-Martin periodinane (10.7 mg, 0.02512 mmol, 1 equiv) and $\mathrm{NaHCO}_{3}(8.8 \mathrm{mg}, 0.1046 \mathrm{mmol}, 1$ equiv). After 1.5 hours, $1 \mathrm{M} \mathrm{KOH}(0.5 \mathrm{~mL})$ was added to mixed solution. After 12 hours, the resulting reaction mixture was diluted with ethyl acetate $(10 \mathrm{~mL})$ and saturated aqueous sodium thiosulfate solution $(10 \mathrm{~mL})$ and the layers were separated. The aqueous layer was extracted with ethyl acetate $(3 \times 10 \mathrm{~mL})$, and the combined organic layers were dried over anhydrous sodium sulfate and concentrated under reduced pressure. The resulting crude residue was purified by column chromatography (silica gel: diam. $2 \mathrm{~cm}$, ht. $9 \mathrm{~cm}$; eluent : ethyl acetate : $\left.\mathrm{NH}_{4} \mathrm{OH}=100: 1\right)$ to afford $6(4.5 \mathrm{mg}, 3$ steps $64 \%)$ as a yellow solid.

${ }^{1} \mathrm{H}$ NMR $\left(400 \mathrm{MHz}, \mathrm{CDCl}_{3}\right): \delta 7.47(\mathrm{~d}, \mathrm{~J}=8.2 \mathrm{~Hz}, 1 \mathrm{H}), 7.44(\mathrm{~d}, \mathrm{~J}=7.8 \mathrm{~Hz}, 1 \mathrm{H}), 7.29-7.21(\mathrm{~m}, 1 \mathrm{H})$, 7.16 (ddd, J = 8.0, 7.1, 1.0 Hz, 1H), $7.07-7.02(\mathrm{~m}, 1 \mathrm{H}), 5.87(\mathrm{t}, \mathrm{J}=7.1 \mathrm{~Hz}, 1 \mathrm{H}), 5.69(\mathrm{~d}, \mathrm{~J}=7.0 \mathrm{~Hz}$, $1 \mathrm{H}$ ), 3.75 (dddd, $\mathrm{J}=14.7,12.6,6.4,2.2 \mathrm{~Hz}, 1 \mathrm{H}$ ), 3.28 (ddt, $\mathrm{J}=11.3,7.8,3.7 \mathrm{~Hz}, 1 \mathrm{H}), 2.93-2.80(\mathrm{~m}$, $3 \mathrm{H}), 2.77-2.69(\mathrm{~m}, 1 \mathrm{H}), 2.70-2.61(\mathrm{~m}, 1 \mathrm{H}), 2.29(\mathrm{dd}, \mathrm{J}=19.0,2.1 \mathrm{~Hz}, 1 \mathrm{H}), 2.18(\mathrm{~s}, 3 \mathrm{H}), 2.19-2.13$ $(\mathrm{m}, 1 \mathrm{H})$

${ }^{13} \mathrm{C}$ NMR $\left(101 \mathrm{MHz}, \mathrm{CDCl}_{3}\right): \delta 198.3,174.4,139.8,138.1,136.0,128.6,127.7,123.2,120.9,118.6$, $111.4,110.5,80.9,44.2,41.2,32.9,26.6,25.7,25.6,25.5$.

HRMS (ESI): Calculated for $\mathrm{C}_{20} \mathrm{H}_{20} \mathrm{~N}_{2} \mathrm{O}_{3}[\mathrm{M}+\mathrm{Na}]^{+}:$359.1366, found: 359.1370 .

$[\alpha]^{25} \mathrm{D}:-236^{\circ}\left(c=0.08\right.$ in $\left.\mathrm{CHCl}_{3}\right) .^{12}$

TLC (ethyl acetate) Rf: 0.3 (UV). 


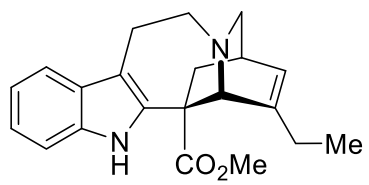

(+)-catharanthine (3)

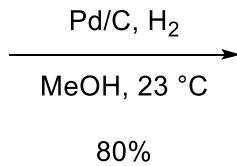

$\mathrm{KH}, \mathrm{ClCO}_{2} \mathrm{Me}$

THF, 0 to $23^{\circ} \mathrm{C}$

$83 \%$

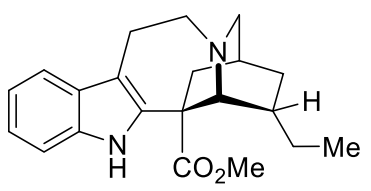

(+)-dihydrocatharanthine (S10)
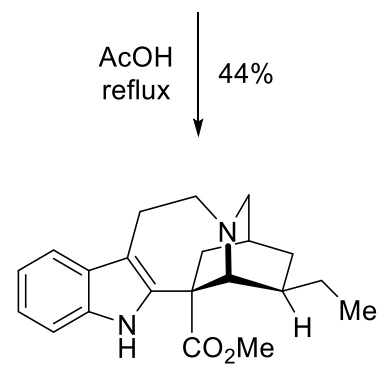

(+)-coronaridine (S11)

1. NIS, TFA

$\mathrm{MeNO}_{2},-40^{\circ} \mathrm{C}$

2. $\mathrm{K}_{2} \mathrm{CO}_{3}$

$\mathrm{MeOH}, 23^{\circ} \mathrm{C}$

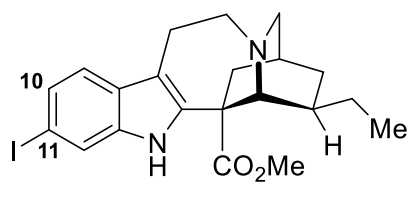

S13

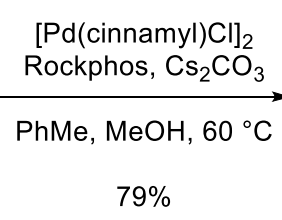

$79 \%$

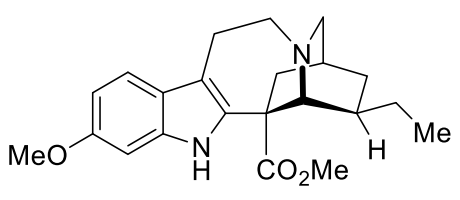

(+)-isovoacangine (44)

Figure S1. Synthesis of (+)-isovoacangine (44) from (+)-catharanthine (3). 
<smiles>CCC1CN2CCCc3c([nH]c4ccccc34)C2(C(C)OC)C1</smiles>

(+)-dihydrocatharanthine (S10)

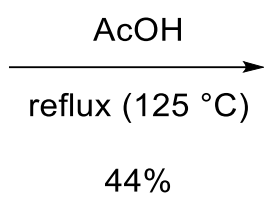

$44 \%$

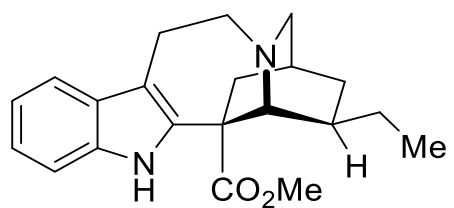

$(+)$-coronaridine (S11)

\section{(+)-coronaridine (S11):}

To a stirred solution of $\mathbf{S} 10^{13}$ (1.6 g, $4.739 \mathrm{mmol}, 1$ equiv) in acetic acid (30 mL) at room temperature and the temperature was raised to $125^{\circ} \mathrm{C}$. After 48 hours, the resulting crude residue was concentrated and dissolved in dichloromethane $(20 \mathrm{~mL})$ and saturated aqueous sodium bicarbonate solution $(20 \mathrm{~mL})$ and the layers were separated. The aqueous layer was extracted with dichloromethane $(3 \times 20 \mathrm{~mL})$, and the combined organic layers were dried over anhydrous sodium sulfate and concentrated under reduced pressure. The resulting crude residue was purified by column chromatography (silica gel: diam. $5 \mathrm{~cm}$, ht. $9 \mathrm{~cm}$; eluent : hexane : ethyl acetate $=5: 1$ ) to afford $\mathbf{S 1 1}$ (702 $\mathrm{mg}, 44 \%$ ) as a yellow solid.

${ }^{1} \mathrm{H}$ NMR $\left(500 \mathrm{MHz}, \mathrm{CDCl}_{3}\right): \delta 7.80(\mathrm{~s}, 1 \mathrm{H}), 7.47(\mathrm{~d}, \mathrm{~J}=7.8 \mathrm{~Hz}, 1 \mathrm{H}), 7.23(\mathrm{~d}, \mathrm{~J}=7.4 \mathrm{~Hz}, 1 \mathrm{H}), 7.13$ (ddd, $\mathrm{J}=8.1,7.0,1.2 \mathrm{~Hz}, 1 \mathrm{H}), 7.07$ (ddd, $\mathrm{J}=8.0,6.9,1.1 \mathrm{~Hz}, 1 \mathrm{H}), 3.70(\mathrm{~s}, 3 \mathrm{H}), 3.55(\mathrm{~s}, 1 \mathrm{H}), 3.38$ (ddd, $\mathrm{J}=$ 15.3, 8.6, $5.8 \mathrm{~Hz}, 1 \mathrm{H}$ ), 3.19 (dddd, $\mathrm{J}=13.8,12.1,7.6,5.9 \mathrm{~Hz}, 2 \mathrm{H}), 3.05-2.96(\mathrm{~m}, 1 \mathrm{H}), 2.90$ (dt, J = 8.5, 3.0 Hz, 1H), $2.80(\mathrm{~d}, \mathrm{~J}=8.6 \mathrm{~Hz}, 1 \mathrm{H}), 2.60-2.54(\mathrm{~m}, 1 \mathrm{H}), 1.93-1.85(\mathrm{~m}, 2 \mathrm{H}), 1.73$ (dddd, J = 12.2, 9.8, 4.3, $2.1 \mathrm{~Hz}, 1 \mathrm{H}), 1.57(\mathrm{dt}, \mathrm{J}=13.3,7.4 \mathrm{~Hz}, 1 \mathrm{H}), 1.43$ (ddd, J = 13.6, 7.6, 6.4 Hz, $1 \mathrm{H}), 1.32$ (dq, J = 9.4, 7.1 Hz, 1H), $1.15-1.09(\mathrm{~m}, 1 \mathrm{H}), 0.89(\mathrm{t}, \mathrm{J}=7.4 \mathrm{~Hz}, 3 \mathrm{H})$.

${ }^{13} \mathrm{C}$ NMR $\left(101 \mathrm{MHz}, \mathrm{CDCl}_{3}\right): \delta 175.8,136.6,135.5,128.8,122.0,119.2,118.5,110.4,110.3,57.5,55.1$, $53.2,52.6,51.6,39.2,36.5,32.1,27.4,26.8,22.1,11.7$.

HRMS (ESI): Calculated for $\mathrm{C}_{21} \mathrm{H}_{26} \mathrm{~N}_{2} \mathrm{O}_{2}[\mathrm{M}+\mathrm{H}]^{+}: 339.2067$, found: 339.2055 .

$[\alpha]^{25} \mathrm{D}:+2^{\circ}(\mathrm{HCl}$ salt, $c=1$ in $\mathrm{MeOH}) \cdot{ }^{14}$

TLC (hexane : ethyl acetate $=4: 1$ ) Rf: 0.5 (UV). 
<smiles>CC[C@H]1CN2CCc3c([nH]c4ccccc34)C1(C(O)OC)C2</smiles>

(+)-coronaridine (S11)

\author{
$\underset{\mathrm{THF}, 0 \text { to } 23{ }^{\circ} \mathrm{C}}{\stackrel{\mathrm{KH}, \mathrm{ClCO}_{2} \mathrm{Me}}{\longrightarrow}}$
}

$83 \%$<smiles>CCC1CC2CN(CCc3c2n(COC)c2ccccc32)C1(COC)C(=O)OC</smiles>

S12

\section{methylcarbamate S12:}

To a stirred solution of potassium hydride ( $250 \mathrm{mg}, 6.220 \mathrm{mmol}, 3$ equiv) in THF (10 mL) was added S11 solution (702 mg, $2.0733 \mathrm{mmol}, 1$ equiv) in THF $10 \mathrm{~mL}$ at $0{ }^{\circ} \mathrm{C}$. After $30 \mathrm{~min}$, methyl chloroformate $(0.64 \mathrm{~mL}, 8.293 \mathrm{mmol}, 4$ equiv) was added dropwise. The mixture was further stirred for 1 hour at $0{ }^{\circ} \mathrm{C}$ and the temperature was raised to room temperature. After 12 hours, the resulting crude residue was dissolved in ethyl acetate $(20 \mathrm{~mL})$ and saturated aqueous sodium bicarbonate solution $(20$ $\mathrm{mL})$ and the layers were separated. The aqueous layer was extracted with dichloromethane $(3 \times 20$ $\mathrm{mL}$ ), and the combined organic layers were dried over anhydrous sodium sulfate and concentrated under reduced pressure. The resulting crude residue was purified by column chromatography (silica gel: diam. $5 \mathrm{~cm}$, ht. $9 \mathrm{~cm}$; eluent : hexane : ethyl acetate $=5: 1)$ to afford $\mathbf{S} 12(685.6 \mathrm{mg}, 83 \%)$ as a yellow solid.

${ }^{1} \mathrm{H}$ NMR $\left(400 \mathrm{MHz}, \mathrm{CDCl}_{3}\right): \delta 8.08(\mathrm{dd}, \mathrm{J}=7.4,1.2 \mathrm{~Hz}, 1 \mathrm{H}), 7.48(\mathrm{dd}, \mathrm{J}=7.1,1.6 \mathrm{~Hz}, 1 \mathrm{H}), 7.32-7.22$ $(\mathrm{m}, 2 \mathrm{H}), 3.92(\mathrm{~s}, 3 \mathrm{H}), 3.61(\mathrm{~s}, 3 \mathrm{H}), 3.56-3.48(\mathrm{~m}, 2 \mathrm{H}), 3.19$ (ddd, J = 15.3, 13.5, $6.4 \mathrm{~Hz}, 1 \mathrm{H}), 3.03$ (ddd, J = 8.2, 3.7, 2.6 Hz, 1H), 2.91 (ddd, J = 15.4, 5.1, $1.6 \mathrm{~Hz}, 1 \mathrm{H}$ ), $2.77-2.65(\mathrm{~m}, 2 \mathrm{H}), 2.54$ (d, J = $8.2 \mathrm{~Hz}, 1 \mathrm{H}$ ), 1.88 (dddd, $\mathrm{J}=23.6,11.8,9.8,4.3 \mathrm{~Hz}, 2 \mathrm{H}), 1.78$ (dt, J = 3.9, $2.0 \mathrm{~Hz}, 1 \mathrm{H}), 1.72$ (ddd, J = 13.6, 4.3, $2.5 \mathrm{~Hz}, 1 \mathrm{H}), 1.53-1.40(\mathrm{~m}, 2 \mathrm{H}), 1.06$ (ddt, $\mathrm{J}=11.8,6.4,2.2 \mathrm{~Hz}, 1 \mathrm{H}), 0.88(\mathrm{t}, \mathrm{J}=7.4 \mathrm{~Hz}, 3 \mathrm{H})$.

${ }^{13} \mathrm{C}$ NMR $\left(101 \mathrm{MHz}, \mathrm{CDCl}_{3}\right): \delta 174.5,152.1,139.1,136.1,129.8,124.5,122.8,119.0,118.3,115.5$, $57.2,56.0,55.1,53.4,52.8,52.4,36.9,36.6,32.1,28.3,27.5,22.1,11.8$.

HRMS (ESI): Calculated for $\mathrm{C}_{23} \mathrm{H}_{28} \mathrm{~N}_{2} \mathrm{O}_{4}[\mathrm{M}+\mathrm{Na}]^{+}:$419.1941, found: 419.1945.

$[\alpha]^{25} \mathrm{D}:+47^{\circ}\left(\mathrm{C}=1.0\right.$ in $\left.\mathrm{CHCl}_{3}\right)$.

TLC (hexane : ethyl acetate $=4: 1$ ) Rf: 0.55 (UV). 


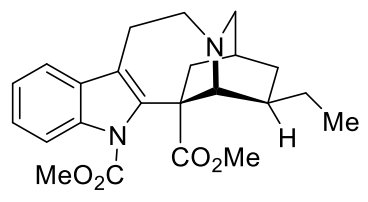

S12

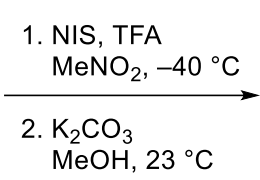

$\mathrm{MeOH}, 23^{\circ} \mathrm{C}$

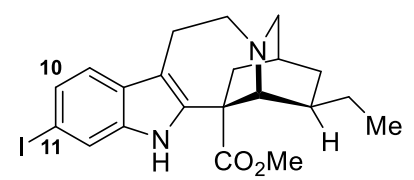

S13

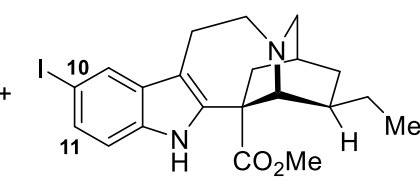

S14

$35 \%$ (2 steps)

$38 \%$ (2 steps)

\section{iodides S13:}

To a stirred solution of $\mathbf{S} 12$ (170.2 $\mathrm{mg}, 0.4293 \mathrm{mmol}, 1$ equiv) in TFA (30 mL) and $\mathrm{MeNO}_{2}(30$ $\mathrm{mL}$ ) was added $\mathrm{N}$-iodosuccinimide $\left(145 \mathrm{mg}, 0.6439 \mathrm{mmol}, 1.5\right.$ equiv) at $-40^{\circ} \mathrm{C}$. After 12 hours, the resulting reaction mixture was diluted with ethyl acetate $(20 \mathrm{~mL})$ and saturated aqueous sodium thiosulfate solution $(20 \mathrm{~mL})$ and saturated aqueous sodium carbonate solution $(30 \mathrm{~mL})$ and the layers were separated. The aqueous layer was extracted with ethyl acetate $(3 \times 30 \mathrm{~mL})$, and the combined organic layers were dried over anhydrous sodium sulfate and concentrated under reduced pressure. The resulting crude residue was purified by column chromatography (silica gel: diam. $5 \mathrm{~cm}$, ht. $10 \mathrm{~cm}$; eluent : hexane : ethyl acetate $=100: 3$ ) to afford 10-iodinated compound and 11-iodinated compound, $1: 1$ mixture $(176.8 \mathrm{mg}, 79 \%)$ as a pale yellow solid. To a solution of the mixture (157.6 $\mathrm{mg}, 0.3017$ mmol, 1 equiv) in $\mathrm{MeOH}\left(4 \mathrm{~mL}\right.$ ) was added $\mathrm{K}_{2} \mathrm{CO}_{3}$ (208 mg, $1.5085 \mathrm{mmol}, 5$ equiv). After 12 hours, the resulting reaction mixture was diluted with dichloromethane $(20 \mathrm{~mL})$ and saturated aqueous sodium chloride solution $(20 \mathrm{~mL})$ and the layers were separated. The aqueous layer was extracted with dichloromethane $(3 \times 20 \mathrm{~mL})$, and the combined organic layers were dried over anhydrous sodium sulfate and concentrated under reduced pressure. The resulting crude residue was purified by column chromatography (silica gel: diam. $3 \mathrm{~cm}$, ht. $9 \mathrm{~cm}$; eluent : hexane : ethyl acetate $=100: 3$ ) to afford 11 iodinated S13 (67 mg, 48\%) and 10-iodinated S14 (61 mg, 44\%) as a yellow solid.

${ }^{1} \mathrm{H}$ NMR $\left(500 \mathrm{MHz}, \mathrm{CDCl}_{3}\right): \delta 8.03(\mathrm{~s}, 1 \mathrm{H}), 7.59(\mathrm{~d}, \mathrm{~J}=1.5 \mathrm{~Hz}, 1 \mathrm{H}), 7.34(\mathrm{dd}, \mathrm{J}=8.3,1.5 \mathrm{~Hz}, 1 \mathrm{H}), 7.21$ $(\mathrm{d}, \mathrm{J}=8.3 \mathrm{~Hz}, 1 \mathrm{H}), 3.72(\mathrm{~s}, 3 \mathrm{H}), 3.55(\mathrm{~s}, 1 \mathrm{H}), 3.38(\mathrm{qd}, \mathrm{J}=9.1,8.4,3.8 \mathrm{~Hz}, 1 \mathrm{H}), 3.21-3.09(\mathrm{~m}, 2 \mathrm{H})$, $3.00-2.93(\mathrm{~m}, 1 \mathrm{H}), 2.92-2.88(\mathrm{~m}, 1 \mathrm{H}), 2.76(\mathrm{~d}, \mathrm{~J}=8.4 \mathrm{~Hz}, 1 \mathrm{H}), 2.58(\mathrm{dt}, \mathrm{J}=14.6,3.0 \mathrm{~Hz}, 1 \mathrm{H}), 1.86$ $(\mathrm{dq}, \mathrm{J}=10.2,3.9 \mathrm{~Hz}, 2 \mathrm{H}), 1.77-1.70(\mathrm{~m}, 1 \mathrm{H}), 1.56(\mathrm{dq}, \mathrm{J}=14.7,7.4 \mathrm{~Hz}, 1 \mathrm{H}), 1.45(\mathrm{dp}, \mathrm{J}=14.0,7.2$ $\mathrm{Hz}, 1 \mathrm{H}), 1.34$ (dt, J = 16.0, $7.2 \mathrm{~Hz}, 1 \mathrm{H}), 1.16-1.08(\mathrm{~m}, 1 \mathrm{H}), 0.91(\mathrm{t}, \mathrm{J}=7.4 \mathrm{~Hz}, 3 \mathrm{H})$.

${ }^{13} \mathrm{C}$ NMR $\left(126 \mathrm{MHz}, \mathrm{CDCl}_{3}\right): \delta$ 175.8, 137.3, 137.0, 128.4, 128.1, 120.3, 119.5, 110.7, 85.8, 57.6, 55.2, $53.1,52.9,51.8,39.2,36.6,32.1,27.5,26.9,22.1,11.8$.

HRMS (ESI): Calculated for $\mathrm{C}_{21} \mathrm{H}_{25} \mathrm{IN}_{2} \mathrm{O}_{2}[\mathrm{M}+\mathrm{H}]^{+}:$465.1033, found: 465.1026 .

$[\alpha]^{25} \mathrm{D}:+23^{\circ}\left(c=1\right.$ in $\left.\mathrm{CHCl}_{3}\right)$. TLC (hexane : ethyl acetate $\left.=4: 1\right) \mathrm{Rf}: 0.55$ (UV).

\section{iodides S14:}

${ }^{1} \mathrm{H}$ NMR $\left(500 \mathrm{MHz}, \mathrm{CDCl}_{3}\right): \delta 7.89(\mathrm{~s}, 1 \mathrm{H}), 7.78(\mathrm{~s}, 1 \mathrm{H}), 7.36(\mathrm{dd}, \mathrm{J}=8.5,1.6 \mathrm{~Hz}, 1 \mathrm{H}), 7.01(\mathrm{~d}, \mathrm{~J}=8.4$ $\mathrm{Hz}, 1 \mathrm{H}), 3.70(\mathrm{~s}, 3 \mathrm{H}), 3.52(\mathrm{~s}, 1 \mathrm{H}), 3.36(\mathrm{ddd}, \mathrm{J}=13.4,7.7,5.9 \mathrm{~Hz}, 1 \mathrm{H}), 3.19-3.05(\mathrm{~m}, 2 \mathrm{H}), 2.97-$ $2.87(\mathrm{~m}, 2 \mathrm{H}), 2.74(\mathrm{~d}, \mathrm{~J}=8.4 \mathrm{~Hz}, 1 \mathrm{H}), 2.56(\mathrm{dt}, \mathrm{J}=14.7,3.0 \mathrm{~Hz}, 1 \mathrm{H}), 1.86(\mathrm{dq}, \mathrm{J}=7.0,3.3,2.6 \mathrm{~Hz}, 2 \mathrm{H})$, $1.71(\mathrm{dt}, \mathrm{J}=12.0,6.4 \mathrm{~Hz}, 1 \mathrm{H}), 1.55(\mathrm{dt}, \mathrm{J}=13.3,7.4 \mathrm{~Hz}, 1 \mathrm{H}), 1.42(\mathrm{dp}, \mathrm{J}=14.2,7.2 \mathrm{~Hz}, 1 \mathrm{H}), 1.31$ (dq, $\mathrm{J}=9.7,7.2 \mathrm{~Hz}, 1 \mathrm{H}), 1.11$ (ddt, $\mathrm{J}=12.1,7.3,2.2 \mathrm{~Hz}, 1 \mathrm{H}), 0.88(\mathrm{t}, \mathrm{J}=7.4 \mathrm{~Hz}, 3 \mathrm{H})$.

${ }^{13} \mathrm{C}$ NMR $\left(126 \mathrm{MHz}, \mathrm{CDCl}_{3}\right): \delta 175.7,137.8,134.7,131.6,130.3,127.7,112.5,110.0,82.7,57.5,55.2$, $53.1,52.9,51.9,39.3,36.8,32.2,27.5,26.9,22.2,11.9$.

HRMS (ESI): Calculated for $\mathrm{C}_{21} \mathrm{H}_{25} \mathrm{IN}_{2} \mathrm{O}_{2}[\mathrm{M}+\mathrm{H}]^{+}:$465.1033, found: 465.1032 .

$[\alpha]^{25}$ : $+24^{\circ}\left(c=1\right.$ in $\left.\mathrm{CHCl}_{3}\right)$. TLC (hexane : ethyl acetate $\left.=4: 1\right) \mathrm{Rf}: 0.50$ (UV). 
<smiles>CCC1CN2CCC1[C@@H]1CN(C2)CC1(COC)C(=O)O</smiles>

$\mathbf{S 1 3}$

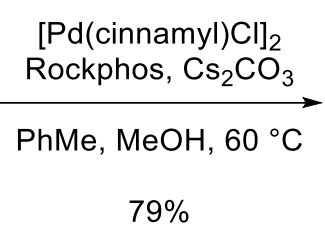

$79 \%$<smiles>CCC1CN2CCC1[C@@H]1CN(C2)CC1(COC)C(C)C</smiles>

(+)-isovoacangine (44)

\section{(+)-isovoacangine (44):}

To a solution of $\mathbf{S 1 3}(38.9 \mathrm{mg}, 0.0838 \mathrm{mmol}, 1$ equiv) in toluene $(2 \mathrm{~mL})$ was added $\left[\mathrm{Pd}\left(\right.\right.$ cinnamyl) $\mathrm{Cl}_{2}(13 \mathrm{mg}, 0.0251 \mathrm{mmol}, 0.3$ equiv), Rockphos (23.6 mg, $0.0503 \mathrm{mmol}, 0.6$ equiv), $\mathrm{Cs}_{2} \mathrm{CO}_{3}$ (68.2 mg, $0.2094 \mathrm{mmol}, 2.5$ equiv) and methanol ( $0.034 \mathrm{~mL}, 0.8377 \mathrm{mmol}, 10.0$ equiv) in glove box and raised temperature to $60^{\circ} \mathrm{C}$. After 12 hours, the crude product was filtered through a pad of celite, washed with ethyl acetate $(10 \mathrm{~mL})$ and the resulting mixture was diluted with ethyl acetate (10 $\mathrm{mL}$ ) and saturated aqueous sodium chloride solution $(10 \mathrm{~mL})$ and the layers were separated. The aqueous layer was extracted with ethyl acetate $(3 \times 10 \mathrm{~mL})$, and the combined organic layers were dried over anhydrous sodium sulfate and concentrated under reduced pressure. The resulting crude residue was purified by column chromatography (silica gel: diam. $3 \mathrm{~cm}$, ht. $9 \mathrm{~cm}$; eluent : hexane : ethyl acetate $=15: 1)$ to afford $44(24.4 \mathrm{mg}, 79 \%)$ as a yellow solid.

${ }^{1} \mathrm{H}$ NMR $\left(400 \mathrm{MHz}, \mathrm{CDCl}_{3}\right): \delta 7.67(\mathrm{~s}, 1 \mathrm{H}), 7.31(\mathrm{~d}, \mathrm{~J}=9.3 \mathrm{~Hz}, 1 \mathrm{H}), 6.77-6.70(\mathrm{~m}, 2 \mathrm{H}), 3.81(\mathrm{~s}, 3 \mathrm{H})$, $3.70(\mathrm{~s}, 3 \mathrm{H}), 3.51(\mathrm{~s}, 1 \mathrm{H}), 3.40-3.30(\mathrm{~m}, 1 \mathrm{H}), 3.18(\mathrm{~d}, \mathrm{~J}=17.3 \mathrm{~Hz}, 1 \mathrm{H}), 3.12(\mathrm{td}, \mathrm{J}=8.2,6.0 \mathrm{~Hz}, 1 \mathrm{H})$, $2.99-2.89(\mathrm{~m}, 1 \mathrm{H}), 2.88(\mathrm{~d}, \mathrm{~J}=7.0 \mathrm{~Hz}, 1 \mathrm{H}), 2.80(\mathrm{~d}, \mathrm{~J}=8.4 \mathrm{~Hz}, 1 \mathrm{H}), 2.58-2.48(\mathrm{~m}, 1 \mathrm{H}), 1.91-1.83$ $(\mathrm{m}, 2 \mathrm{H}), 1.77-1.64(\mathrm{~m}, 1 \mathrm{H}), 1.61-1.49(\mathrm{~m}, 1 \mathrm{H}), 1.47-1.35(\mathrm{~m}, 1 \mathrm{H}), 1.34-1.24(\mathrm{~m}, 1 \mathrm{H}), 1.12(\mathrm{~d}, \mathrm{~J}$ $=11.9 \mathrm{~Hz}, 1 \mathrm{H}), 0.88(\mathrm{t}, \mathrm{J}=7.4 \mathrm{~Hz}, 3 \mathrm{H})$.

${ }^{13}$ C NMR $\left(101 \mathrm{MHz}, \mathrm{CDCl}_{3}\right): \delta$ 176.1, 156.7, 136.4, 135.4, 123.4, 119.3, 110.3, 109.2, 94.4, 57.9, 55.9, $55.2,53.3,52.8,51.6,39.4,36.6,32.3,27.6,26.9,22.4,11.9$.

HRMS (ESI): Calculated for $\mathrm{C}_{22} \mathrm{H}_{28} \mathrm{~N}_{2} \mathrm{O}_{3}[\mathrm{M}+\mathrm{H}]^{+}: 369.2173$, found: 369.2173 .

$[\alpha]^{25} \mathrm{D}:+33^{\circ}\left(c=1\right.$ in $\left.\mathrm{CHCl}_{3}\right){ }^{15}$

TLC (hexane : ethyl acetate $=4: 1$ ) Rf: 0.3 (UV). 


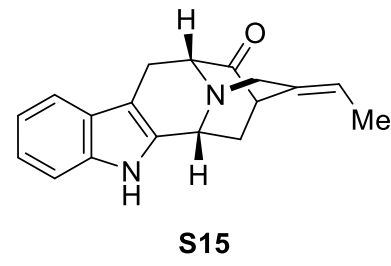

1. $\mathrm{tBuOK}$,

$\left[\mathrm{P}(\mathrm{Ph})_{3} \mathrm{CH}_{2} \mathrm{OMe}\right] \mathrm{Cl}$

$\mathrm{PhH}, \mathrm{THF}, 23^{\circ} \mathrm{C}$

2. $2 \mathrm{~N} \mathrm{HCl}, \mathrm{THF}, 55^{\circ} \mathrm{C}$

3. $\mathrm{KOH}, \mathrm{I}_{2}, \mathrm{MeOH}, 0^{\circ} \mathrm{C}$

$28 \%$ (3 steps)

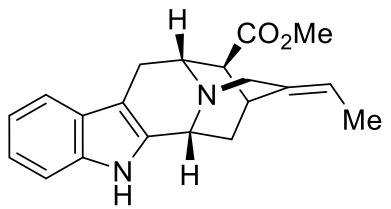

(-)-16-epi-pericyclivine (7)

\section{(-)-16-epi-pericyclivine (7):}

A mixture of anhydrous tBuOK (3.75 g, $33.39 \mathrm{mmol}, 6$ equiv) and $\left[\mathrm{P}(\mathrm{Ph})_{3} \mathrm{CH}_{2} \mathrm{OMe}\right] \mathrm{Cl}(9.54 \mathrm{~g}$, $27.83 \mathrm{mmol}, 5$ equiv) in dry benzene $(50 \mathrm{~mL})$ was allowed to stir at room temperature for 1 hour. The pentacyclic ketone $\mathbf{S} 15^{16}$ (1.55g, $5.5655 \mathrm{mmol}$, 1 equiv) in THF $(50 \mathrm{~mL})$ was then added to the above orange solution dropwise at room temperature. The mixture that resulted was stirred at room temperature for 12 hours. The mixture was diluted with ethyl acetate $(100 \mathrm{~mL})$ and saturated aqueous sodium chloride solution $(100 \mathrm{~mL})$ and the layers were separated. The aqueous layer was extracted with ethyl acetate $(3 \times 10 \mathrm{~mL})$, and the combined organic layers were dried over anhydrous sodium sulfate and concentrated under reduced pressure. The residue was dissolved (without further purification) in a solution of aqueous $\mathrm{HCl}(2 \mathrm{~N})$ in water and $\operatorname{THF}(1: 1,100 \mathrm{~mL})$. The solution that resulted was stirred at $55^{\circ} \mathrm{C}$ under an atmosphere of argon for 12 hours. The reaction mixture was cooled to $0{ }^{\circ} \mathrm{C}$ and extracted with ethyl ether $(3 \times 100 \mathrm{~mL})$ to remove phosphorus-based byproducts, and the water layer was then brought to $\mathrm{pH} 8$ with and ice-cold aqueous solution of $\mathrm{NaOH}(1 \mathrm{~N})$. The aqueous layer was extracted with dichloromethane $(3 \times 100 \mathrm{~mL})$, and the combined organic layers were washed with brine $(200 \mathrm{~mL})$ and dried over anhydrous sodium sulfate and concentrated under reduced pressure. The residue was dissolved (without further purification) in anhydrous methanol $(40 \mathrm{~mL})$ and a solution of $6 \mathrm{M} \mathrm{KOH}$ in anhydrous methanol $(3.7 \mathrm{~mL}, 22.2618 \mathrm{mmol}, 4$ equiv) and iodine ( $3.53 \mathrm{~g}, 13.9136 \mathrm{mmol}$, 2.5 equiv) in anhydrous methanol $(15 \mathrm{~mL})$ was added dropwise at $0{ }^{\circ} \mathrm{C}$. After 2 hours, the reaction was quenched by adding saturated sodium thiosulfate $(100 \mathrm{~mL})$. The mixture was diluted with dichloromethane $(100 \mathrm{~mL})$ and saturated aqueous sodium chloride solution $(100 \mathrm{~mL})$ and the layers were separated. The aqueous layer was extracted with dichloromethane $(3 \times 100 \mathrm{~mL})$, and the combined organic layers were dried over anhydrous sodium sulfate and concentrated under reduced pressure. The resulting crude residue was purified by column chromatography (silica gel: diam. $5 \mathrm{~cm}$, ht. $9 \mathrm{~cm}$; eluent : hexane : ethyl acetate $=1: 2)$ to afford $(-)$-16-epi-pericyclivine $(7)(504.7 \mathrm{mg}, 28 \%, 3$ steps) as a yellow solid.

${ }^{1} \mathrm{H}$ NMR $\left(500 \mathrm{MHz}, \mathrm{CDCl}_{3}\right): \delta 7.94(\mathrm{~s}, 1 \mathrm{H}), 7.45(\mathrm{~d}, \mathrm{~J}=7.7 \mathrm{~Hz}, 1 \mathrm{H}), 7.29(\mathrm{~d}, \mathrm{~J}=8.0 \mathrm{~Hz}, 1 \mathrm{H}), 7.13$ (ddd, $\mathrm{J}=8.2,7.0,1.3 \mathrm{~Hz}, 1 \mathrm{H}), 7.07(\mathrm{t}, \mathrm{J}=7.1 \mathrm{~Hz}, 1 \mathrm{H}), 5.30(\mathrm{q}, \mathrm{J}=6.9 \mathrm{~Hz}, 1 \mathrm{H}), 4.13(\mathrm{~d}, \mathrm{~J}=10.1 \mathrm{~Hz}, 1 \mathrm{H})$, $3.67-3.64(\mathrm{~m}, 1 \mathrm{H}), 3.65(\mathrm{~s}, 3 \mathrm{H}), 3.60(\mathrm{~s}, 1 \mathrm{H}), 3.52(\mathrm{dt}, \mathrm{J}=16.6,2.3 \mathrm{~Hz}, 1 \mathrm{H}), 3.16(\mathrm{dd}, \mathrm{J}=4.3,2.2 \mathrm{~Hz}$, $1 \mathrm{H}), 3.12(\mathrm{dd}, \mathrm{J}=15.6,5.1 \mathrm{~Hz}, 1 \mathrm{H}), 2.66(\mathrm{dd}, \mathrm{J}=15.7,1.4 \mathrm{~Hz}, 1 \mathrm{H}), 2.54(\mathrm{dd}, \mathrm{J}=7.7,1.7 \mathrm{~Hz}, 1 \mathrm{H}), 2.04$ $-1.96(\mathrm{~m}, 1 \mathrm{H}), 1.76(\mathrm{dt}, \mathrm{J}=12.7,3.3 \mathrm{~Hz}, 1 \mathrm{H}), 1.57(\mathrm{dt}, \mathrm{J}=6.9,2.0 \mathrm{~Hz}, 3 \mathrm{H})$.

${ }^{13} \mathrm{C}$ NMR $\left(151 \mathrm{MHz}, \mathrm{CDCl}_{3}\right): \delta 174.3,137.9,136.6,134.5,127.8,121.8,119.7,118.4,117.1,111.1$, 104.6, 56.0, 53.1, 51.9, 50.2, 47.1, 33.3, 28.9, 27.4, 12.9 .

HRMS (ESI): Calculated for $\mathrm{C}_{20} \mathrm{H}_{22} \mathrm{~N}_{2} \mathrm{O}_{2}[\mathrm{M}+\mathrm{H}]^{+}: 323.1754$, found: 323.1762 .

$[\alpha]^{25} \mathrm{D}:-4^{\circ}(c=1$ in $\mathrm{MeOH}) \cdot{ }^{17}$ TLC (ethyl acetate) Rf: 0.7 (UV). 


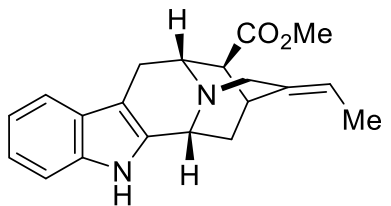

(-)-16-epi-pericyclivine (7)
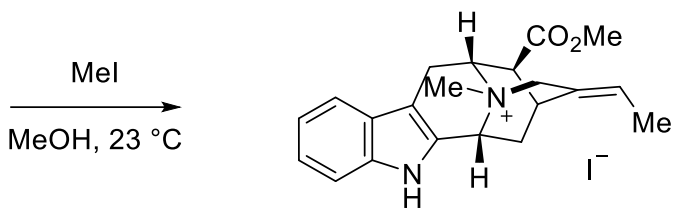

48

\section{ammonium salt 48:}

To a stirred solution of 7 ( $5 \mathrm{mg}, 0.0155 \mathrm{mmol}, 1$ equiv) in methanol (1 mL) was added iodomethane $(50 \mu \mathrm{L})$ at room temperature. After 1.5 hours, the resulting crude residue was concentrated under reduced pressure. $\mathbf{4 8}$ was immediately moved to the next step without further purification.

${ }^{1} \mathrm{H}$ NMR $\left(600 \mathrm{MHz}, \mathrm{CDCl}_{3}\right): \delta 10.33(\mathrm{~s}, 1 \mathrm{H}), 7.51(\mathrm{~d}, \mathrm{~J}=8.4 \mathrm{~Hz}, 1 \mathrm{H}), 7.41(\mathrm{~d}, \mathrm{~J}=7.9 \mathrm{~Hz}, 1 \mathrm{H}), 7.20$ (t, J $=7.7 \mathrm{~Hz}, 1 \mathrm{H}), 7.11(\mathrm{t}, \mathrm{J}=7.5 \mathrm{~Hz}, 1 \mathrm{H}), 5.92(\mathrm{~d}, \mathrm{~J}=10.1 \mathrm{~Hz}, 1 \mathrm{H}), 5.51(\mathrm{~d}, \mathrm{~J}=7.3 \mathrm{~Hz}, 1 \mathrm{H}), 4.89(\mathrm{~d}, \mathrm{~J}=$ $15.7 \mathrm{~Hz}, 1 \mathrm{H}), 4.29(\mathrm{t}, \mathrm{J}=6.3 \mathrm{~Hz}, 1 \mathrm{H}), 4.19(\mathrm{~d}, \mathrm{~J}=15.6 \mathrm{~Hz}, 1 \mathrm{H}), 3.71(\mathrm{~s}, 3 \mathrm{H}), 3.45(\mathrm{~d}, \mathrm{~J}=4.3 \mathrm{~Hz}, 1 \mathrm{H})$, $3.38-3.33(\mathrm{~m}, 1 \mathrm{H}), 3.32(\mathrm{~s}, 3 \mathrm{H}), 2.94(\mathrm{~d}, \mathrm{~J}=17.3 \mathrm{~Hz}, 1 \mathrm{H}), 2.88(\mathrm{~d}, \mathrm{~J}=7.6 \mathrm{~Hz}, 1 \mathrm{H}), 2.76(\mathrm{t}, \mathrm{J}=12.1$ $\mathrm{Hz}, 1 \mathrm{H}), 2.24(\mathrm{~d}, \mathrm{~J}=10.2 \mathrm{~Hz}, 1 \mathrm{H}), 1.59(\mathrm{~s}, 3 \mathrm{H})$.

${ }^{13} \mathrm{C}$ NMR $\left(151 \mathrm{MHz}, \mathrm{CDCl}_{3}\right): \delta 170.7,137.3,131.1,129.0,123.6,122.5,120.6,118.2,112.8,111.3$, 99.5, 65.6, 63.5, 60.4, 53.1, 48.5, 47.1, 32.1, 27.7, 24.7, 12.9 .

HRMS (ESI): Calculated for $\mathrm{C}_{21} \mathrm{H}_{25} \mathrm{IN}_{2} \mathrm{O}_{2}[\mathrm{M}]+: 337.1911$, found: 337.1914 .

TLC (dichloromethane : methanol = $9: 1$ ) Rf: $0.5(U V)$. 


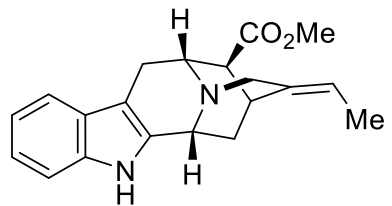

(-)-16-epi-pericyclivine (7)

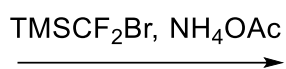

DCE, $23^{\circ} \mathrm{C}$

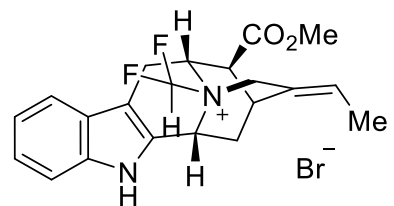

S16

\section{ammonium salt S16:}

To a stirred solution of 7 (11 mg, $0.0352 \mathrm{mmol}, 1$ equiv) in 1,2-dichloroethane (1 mL) was added $\mathrm{NH}_{4} \mathrm{OAc}$ (11 mg, $0.1409 \mathrm{mmol}, 4$ equiv) and $\mathrm{TMSCF}_{2} \mathrm{Br}(11 \mu \mathrm{L}, 0.0704 \mathrm{mmol}, 2$ equiv) at room temperature. After 1.5 hours, the resulting crude residue was dissolved in dichloromethane $(3 \mathrm{~mL})$ and saturated aqueous sodium bicarbonate solution $(3 \mathrm{~mL})$ and the layers were separated. The aqueous layer was extracted with dichloromethane $(3 \times 3 \mathrm{~mL})$, and the combined organic layers were dried over anhydrous sodium sulfate and concentrated under reduced pressure. S16 was characterized without further purification.

${ }^{1} \mathrm{H}$ NMR $\left(500 \mathrm{MHz}, \mathrm{CDCl}_{3}\right): \delta 11.99(\mathrm{~s}, 1 \mathrm{H}), 7.53(\mathrm{dd}, \mathrm{J}=8.2,0.9 \mathrm{~Hz}, 1 \mathrm{H}), 7.37(\mathrm{dt}, \mathrm{J}=8.0,1.0 \mathrm{~Hz}, 1 \mathrm{H})$, $7.24-7.20(\mathrm{~m}, 1 \mathrm{H}), 7.08(\mathrm{ddd}, \mathrm{J}=8.0,7.1,1.0 \mathrm{~Hz}, 1 \mathrm{H}), 6.80(\mathrm{t}, \mathrm{J}=59.1 \mathrm{~Hz}, 1 \mathrm{H}), 6.24(\mathrm{~d}, \mathrm{~J}=10.0 \mathrm{~Hz}$, $1 \mathrm{H}), 5.64(\mathrm{q}, \mathrm{J}=7.1 \mathrm{~Hz}, 1 \mathrm{H}), 4.61-4.50(\mathrm{~m}, 2 \mathrm{H}), 4.19(\mathrm{~d}, \mathrm{~J}=14.8 \mathrm{~Hz}, 1 \mathrm{H}), 3.75(\mathrm{~s}, 3 \mathrm{H}), 3.54(\mathrm{~d}, \mathrm{~J}=$ $4.5 \mathrm{~Hz}, 1 \mathrm{H}), 3.39(\mathrm{dd}, \mathrm{J}=17.8,4.9 \mathrm{~Hz}, 1 \mathrm{H}), 3.06(\mathrm{~d}, \mathrm{~J}=17.9 \mathrm{~Hz}, 1 \mathrm{H}), 3.02(\mathrm{dd}, \mathrm{J}=7.8,1.8 \mathrm{~Hz}, 1 \mathrm{H})$, 2.98 (ddd, $\mathrm{J}=14.2,10.3,1.7 \mathrm{~Hz}, 1 \mathrm{H}$ ), 2.45 (dd, $\mathrm{J}=14.1,4.6 \mathrm{~Hz}, 1 \mathrm{H}), 1.65$ (dd, $\mathrm{J}=7.0,1.4 \mathrm{~Hz}, 3 \mathrm{H}$ ).

${ }^{13} \mathrm{C}$ NMR $\left(101 \mathrm{MHz}, \mathrm{CDCl}_{3}\right): \delta 170.1,137.4,129.5,125.2,124.3,123.6,122.7,120.3,117.9,113.2$, $112.6(\mathrm{t}, \mathrm{J}=278.3 \mathrm{~Hz}), 99.8,61.6,58.4,53.2,47.0,43.6,31.8,27.8,24.6,13.1$.

${ }^{19} \mathrm{~F}$ NMR $\left(376 \mathrm{MHz}, \mathrm{CDCl}_{3}\right): \delta-113.87(\mathrm{dd}, \mathrm{J}=59.2,17.1 \mathrm{~Hz})$.

TLC (dichloromethane : methanol = $9: 1$ ) Rf: 0.43 (UV). 


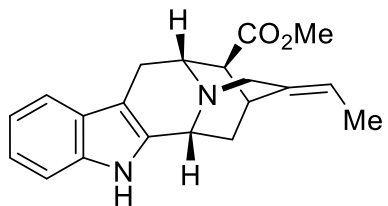

(-)-16-epi-pericyclivine (7)
$\mathrm{TMSCF}_{2} \mathrm{Br}, \mathrm{NH}_{4} \mathrm{OAC}$

DCE, $23^{\circ} \mathrm{C} ; \mathrm{AgBF}_{4}$

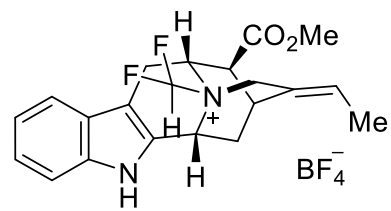

43

\section{ammonium salt 43:}

To a stirred solution of 7 (40.7 $\mathrm{mg}, 0.1262 \mathrm{mmol}, 1$ equiv) in 1,2-dichloroethane (2 mL) was added $\mathrm{NH}_{4} \mathrm{OAc}$ (39 mg, $0.5049 \mathrm{mmol}, 4$ equiv) and $\mathrm{TMSCF}_{2} \mathrm{Br}(79 \mu \mathrm{L}, 0.5049 \mathrm{mmol}, 4$ equiv) at room temperature. After 1.5 hours, $\mathrm{AgBF}_{4}(98.3 \mathrm{mg}, 0.5049 \mathrm{mmol}, 4$ equiv) was added to the reaction mixture. After 1.5 hours, the crude product was filtered through a pad of celite, the resulting crude residue was dissolved in dichloromethane $(3 \mathrm{~mL})$ and saturated aqueous sodium bicarbonate solution $(3 \mathrm{~mL})$ and the layers were separated. The aqueous layer was extracted with dichloromethane $(3 \times 3 \mathrm{~mL})$, and the combined organic layers were dried over anhydrous sodium sulfate and concentrated under reduced pressure. $\mathbf{4 3}$ was immediately moved to the next step without further purification.

${ }^{1} \mathrm{H}$ NMR $\left(500 \mathrm{MHz}, \mathrm{CDCl}_{3}\right): \delta 10.10(\mathrm{~s}, 1 \mathrm{H}), 7.44(\mathrm{~d}, \mathrm{~J}=8.2 \mathrm{~Hz}, 1 \mathrm{H}), 7.39(\mathrm{~d}, \mathrm{~J}=7.9 \mathrm{~Hz}, 1 \mathrm{H}), 7.24-$ $7.20(\mathrm{~m}, 1 \mathrm{H}), 7.08(\mathrm{t}, \mathrm{J}=7.5 \mathrm{~Hz}, 1 \mathrm{H}), 6.65(\mathrm{t}, \mathrm{J}=59.0 \mathrm{~Hz}, 1 \mathrm{H}), 5.67(\mathrm{~d}, \mathrm{~J}=9.1 \mathrm{~Hz}, 2 \mathrm{H}), 4.58(\mathrm{t}, \mathrm{J}=6.3$ $\mathrm{Hz}, 1 \mathrm{H}), 4.35(\mathrm{~d}, \mathrm{~J}=14.9 \mathrm{~Hz}, 1 \mathrm{H}), 4.23(\mathrm{~d}, \mathrm{~J}=15.0 \mathrm{~Hz}, 1 \mathrm{H}), 3.75(\mathrm{~s}, 3 \mathrm{H}), 3.54(\mathrm{~d}, \mathrm{~J}=4.5 \mathrm{~Hz}, 1 \mathrm{H}), 3.38$ $(\mathrm{dd}, \mathrm{J}=18.0,4.9 \mathrm{~Hz}, 1 \mathrm{H}), 3.07(\mathrm{~d}, \mathrm{~J}=17.9 \mathrm{~Hz}, 1 \mathrm{H}), 2.98(\mathrm{dd}, \mathrm{J}=7.8,1.8 \mathrm{~Hz}, 1 \mathrm{H}), 2.82(\mathrm{ddd}, \mathrm{J}=14.0$, 10.2, $1.7 \mathrm{~Hz}, 1 \mathrm{H}), 2.31$ (dd, J = 14.1, 4.8 Hz, $1 \mathrm{H}), 1.65(\mathrm{~d}, \mathrm{~J}=6.5 \mathrm{~Hz}, 3 \mathrm{H})$.

${ }^{13} \mathrm{C}$ NMR $\left(126 \mathrm{MHz}, \mathrm{CDCl}_{3}\right): \delta 170.0,137.5,129.1,125.3,124.7,123.9,122.4,120.6,118.2,113.0$, $112.5,100.5,61.6,58.1,53.3,47.1,43.7,31.3,27.7,24.5,13.1$.

${ }^{19} \mathrm{~F}$ NMR $\left(471 \mathrm{MHz}, \mathrm{CDCl}_{3}\right): \delta-114.4(\mathrm{dd}, \mathrm{J}=113.8,59.0 \mathrm{~Hz}),-151.4,-151.5$.

TLC (dichloromethane : methanol = $9: 1$ ) Rf: 0.2 (UV). 


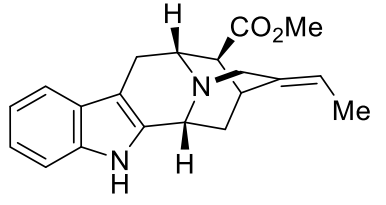

(-)-16-epi-pericyclivine (7)
1. $\mathrm{TMSCF}_{2} \mathrm{Br}, \mathrm{NH}_{4} \mathrm{OAC}$

2.

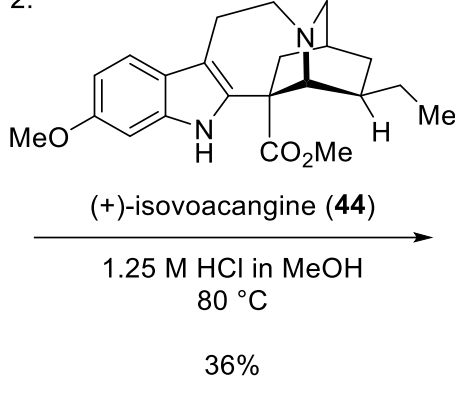

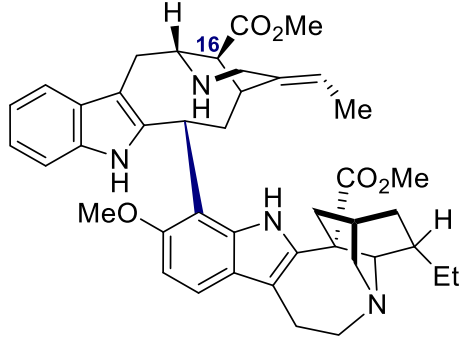

(+)-16-epi-gabunine (8)

\section{6-epi-gabunine (8):}

To a stirred solution of $7(17 \mathrm{mg}, 0.0527 \mathrm{mmol}, 2$ equiv) in 1,2-dichloroethane $(1 \mathrm{~mL})$ in seal tube was added $\mathrm{NH}_{4} \mathrm{OAc}(16.2 \mathrm{mg}, 0.2106 \mathrm{mmol}, 8$ equiv) and TMSCF $2 \mathrm{Br}(33 \mu \mathrm{L}, 0.2106 \mathrm{mmol}, 8$ equiv) at room temperature. After 1.5 hours, $\mathrm{AgBF}_{4}(41 \mathrm{mg}, 0.2106 \mathrm{mmol}, 8$ equiv) was added to the reaction mixture. After 1 hours, the resulting crude residue $\mathbf{4 3}$ was concentrated under reduced pressure.

To a solution of the crude mixture 43 in $1.25 \mathrm{M} \mathrm{HCl}$ in methanol $(1 \mathrm{~mL})$ was added $44(9.7 \mathrm{mg}$, $0.0263 \mathrm{mmol}, 1$ equiv) at room temperature and raised to $80^{\circ} \mathrm{C}$. After 12 hours, the resulting crude residue was dissolved in ethyl acetate $(5 \mathrm{~mL})$ and saturated aqueous sodium bicarbonate solution (10 $\mathrm{mL})$ and the layers were separated. The aqueous layer was extracted with ethyl acetate $(3 \times 10 \mathrm{~mL})$, and the combined organic layers were dried over anhydrous sodium sulfate and concentrated under reduced pressure. the reaction mixture was purified by column chromatography (silica gel: diam. 1 $\mathrm{cm}$, ht. $8 \mathrm{~cm}$; eluent : dichloromethane : methanol $=100: 3)$ to afford $8(6.6 \mathrm{mg}, 36 \%)$ as a yellow solid.

${ }^{1} \mathrm{H}$ NMR $\left(500 \mathrm{MHz}, \mathrm{CDCl}_{3}\right): \delta 7.86(\mathrm{~s}, 1 \mathrm{H}), 7.73(\mathrm{~d}, \mathrm{~J}=7.9 \mathrm{~Hz}, 1 \mathrm{H}), 7.28(\mathrm{~d}, \mathrm{~J}=8.7 \mathrm{~Hz}, 1 \mathrm{H}), 7.17$ (ddd, $\mathrm{J}=8.1,5.9,2.2 \mathrm{~Hz}, 1 \mathrm{H}), 7.13-7.08(\mathrm{~m}, 2 \mathrm{H}), 6.85(\mathrm{~d}, \mathrm{~J}=8.6 \mathrm{~Hz}, 1 \mathrm{H}), 5.35(\mathrm{q}, \mathrm{J}=6.7 \mathrm{~Hz}, 1 \mathrm{H}), 5.10$ (d, J = 12.2 Hz, 1H), $3.99(\mathrm{~s}, 3 \mathrm{H}), 3.93(\mathrm{~d}, \mathrm{~J}=15.3 \mathrm{~Hz}, 1 \mathrm{H}), 3.83-3.77(\mathrm{~m}, 1 \mathrm{H}), 3.71(\mathrm{~d}, \mathrm{~J}=14.0 \mathrm{~Hz}$, $2 \mathrm{H}), 3.68(\mathrm{~s}, 3 \mathrm{H}), 3.64(\mathrm{~s}, 3 \mathrm{H}), 3.62-3.56(\mathrm{~m}, 1 \mathrm{H}), 3.32(\mathrm{~s}, 1 \mathrm{H}), 3.25(\mathrm{dt}, \mathrm{J}=14.5,7.2 \mathrm{~Hz}, 1 \mathrm{H}), 3.10$ (dt, J = 19.9, $7.5 \mathrm{~Hz}, 3 \mathrm{H}), 3.06-2.97(\mathrm{~m}, 1 \mathrm{H}), 2.96-2.92(\mathrm{~m}, 1 \mathrm{H}), 2.85(\mathrm{dt}, \mathrm{J}=16.5,5.7 \mathrm{~Hz}, 1 \mathrm{H}), 2.69$ (s, $1 \mathrm{H}), 2.48(\mathrm{~d}, \mathrm{~J}=8.3 \mathrm{~Hz}, 1 \mathrm{H}), 1.85$ (ddd, $\mathrm{J}=15.0,7.5,2.5 \mathrm{~Hz}, 2 \mathrm{H}), 1.67(\mathrm{~d}, \mathrm{~J}=14.0 \mathrm{~Hz}, 1 \mathrm{H}), 1.60$ (dd, J = 6.7, $1.8 \mathrm{~Hz}, 3 \mathrm{H}), 1.45$ (tq, J = 14.2, 8.3, $6.2 \mathrm{~Hz}, 3 \mathrm{H}), 1.37-1.27(\mathrm{~m}, 2 \mathrm{H}), 1.12(\mathrm{p}, \mathrm{J}=7.5 \mathrm{~Hz}$, $1 \mathrm{H}), 0.96-0.90(\mathrm{~m}, 1 \mathrm{H}), 0.80(\mathrm{t}, \mathrm{J}=7.4 \mathrm{~Hz}, 3 \mathrm{H}), 0.63(\mathrm{dt}, \mathrm{J}=13.6,3.5 \mathrm{~Hz}, 1 \mathrm{H})$.

${ }^{13} \mathrm{C}$ NMR $\left(126 \mathrm{MHz}, \mathrm{CDCl}_{3}\right): \delta 175.4,174.7,152.0,136.6,135.8,135.3,134.9,129.0,124.9,122.4$, $120.0,119.4,118.4,117.6,114.2,110.3,109.7,109.2$, 105.2, 57.9, 57.2, 54.7, 53.1, 52.7, 52.0, 51.6, $51.3,44.5,44.2,39.0,36.6,36.0,35.0,33.4,31.9,29.9,27.3,26.8,24.9,22.1,12.1,11.7$.

HRMS (ESI): Calculated for $\mathrm{C}_{42} \mathrm{H}_{50} \mathrm{~N}_{4} \mathrm{O}_{5}[\mathrm{M}+\mathrm{H}]^{+}: 690.3854$, found: 691.3861 .

$[\alpha]^{25} \mathrm{D}:+21^{\circ}\left(c=0.3\right.$ in $\left.\mathrm{CHCl}_{3}\right)$.

TLC (dichloromethane : methanol = $9: 1$ ) Rf: 0.4 (UV). 


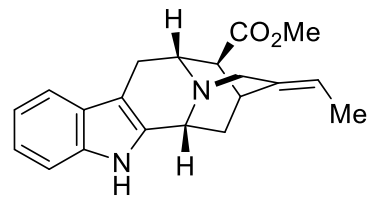

(-)-16-epi-pericyclivine (7)
1. $\mathrm{TMSCF}_{2} \mathrm{Br}, \mathrm{NH}_{4} \mathrm{OAC}$

2. $\mathrm{DCE}, 23^{\circ} \mathrm{C} ; \mathrm{AgBF}_{4}$

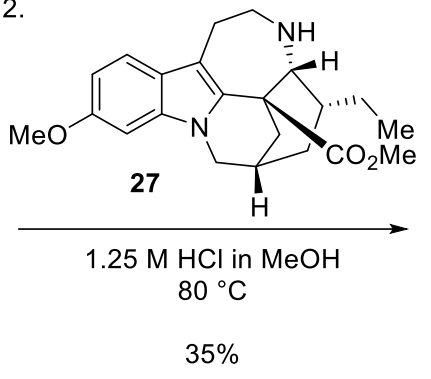

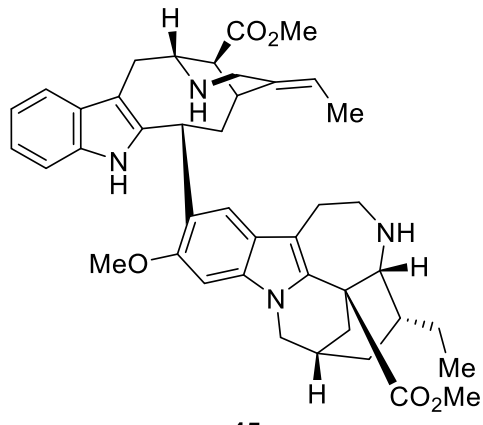

45

\section{heterodimer 45:}

To a stirred solution of 7 ( $8.8 \mathrm{mg}, 0.0271 \mathrm{mmol}, 1$ equiv) in 1,2-dichloroethane (1 mL) in seal tube was added $\mathrm{NH}_{4} \mathrm{OAc}\left(8.4 \mathrm{mg}, 0.1086 \mathrm{mmol}, 4\right.$ equiv) and $\mathrm{TMSCF}_{2} \mathrm{Br}(17 \mu \mathrm{L}, 0.1086 \mathrm{mmol}, 4$ equiv) at room temperature. After 1.5 hours, $\mathrm{AgBF}_{4}(21.1 \mathrm{mg}, 0.1086 \mathrm{mmol}, 4$ equiv) was added to the reaction mixture. After 1 hours, the resulting crude residue $\mathbf{4 3}$ was concentrated under reduced pressure.

To a solution of the crude mixture 43 in $1.25 \mathrm{M} \mathrm{HCl}$ in methanol $(1 \mathrm{~mL})$ was added $27(10 \mathrm{mg}$, $0.0271 \mathrm{mmol}, 1$ equiv) at room temperature and raised to $80^{\circ} \mathrm{C}$. After 12 hours, the resulting crude residue was dissolved in ethyl acetate $(5 \mathrm{~mL})$ and saturated aqueous sodium bicarbonate solution (10 $\mathrm{mL})$ and the layers were separated. The aqueous layer was extracted with ethyl acetate $(3 \times 10 \mathrm{~mL})$, and the combined organic layers were dried over anhydrous sodium sulfate and concentrated under reduced pressure. the reaction mixture was purified by column chromatography (silica gel: diam. $1 \mathrm{~cm}$, ht. $8 \mathrm{~cm}$; eluent : ethyl acetate : methanol = $15: 1)$ to afford $\mathbf{4 5}(6.6 \mathrm{mg}, 35 \%)$ as a yellow solid.

${ }^{1} \mathrm{H}$ NMR $\left(500 \mathrm{MHz}, \mathrm{CDCl}_{3}\right): \delta 7.88(\mathrm{~s}, 1 \mathrm{H}), 7.57(\mathrm{t}, \mathrm{J}=4.5 \mathrm{~Hz}, 1 \mathrm{H}), 7.13-7.08(\mathrm{~m}, 1 \mathrm{H}), 7.06$ (dd, J = $6.1,3.0 \mathrm{~Hz}, 2 \mathrm{H}), 7.01(\mathrm{~s}, 1 \mathrm{H}), 6.80(\mathrm{~s}, 1 \mathrm{H}), 5.37(\mathrm{~d}, \mathrm{~J}=7.0 \mathrm{~Hz}, 1 \mathrm{H}), 4.91(\mathrm{~d}, \mathrm{~J}=11.9 \mathrm{~Hz}, 1 \mathrm{H}), 4.07(\mathrm{~d}$, $\mathrm{J}=3.5 \mathrm{~Hz}, 1 \mathrm{H}), 4.04(\mathrm{~s}, 3 \mathrm{H}), 3.86(\mathrm{~d}, \mathrm{~J}=6.6 \mathrm{~Hz}, 1 \mathrm{H}), 3.81(\mathrm{~d}, \mathrm{~J}=11.2 \mathrm{~Hz}, 1 \mathrm{H}), 3.78-3.73(\mathrm{~m}, 2 \mathrm{H})$, $3.72(\mathrm{~s}, 1 \mathrm{H}), 3.62(\mathrm{~s}, 3 \mathrm{H}), 3.61(\mathrm{~s}, 3 \mathrm{H}), 3.44(\mathrm{dd}, \mathrm{J}=12.3,5.4 \mathrm{~Hz}, 2 \mathrm{H}), 3.22-3.16(\mathrm{~m}, 1 \mathrm{H}), 3.11(\mathrm{~d}, \mathrm{~J}$ $=12.5 \mathrm{~Hz}, 1 \mathrm{H}), 3.06(\mathrm{~s}, 1 \mathrm{H}), 3.01-2.89(\mathrm{~m}, 1 \mathrm{H}), 2.79-2.72(\mathrm{~m}, 1 \mathrm{H}), 2.57(\mathrm{dt}, \mathrm{J}=14.2,5.1 \mathrm{~Hz}, 1 \mathrm{H})$, $2.49-2.41(\mathrm{~m}, 1 \mathrm{H}), 2.28(\mathrm{t}, \mathrm{J}=7.5 \mathrm{~Hz}, 1 \mathrm{H}), 2.21(\mathrm{dd}, \mathrm{J}=12.7,5.4 \mathrm{~Hz}, 1 \mathrm{H}), 2.02(\mathrm{~s}, 1 \mathrm{H}), 1.93(\mathrm{dd}, \mathrm{J}=$ 14.6, 7.0 Hz, 1H), $1.86(\mathrm{~d}, \mathrm{~J}=13.1 \mathrm{~Hz}, 1 \mathrm{H}), 1.78(\mathrm{~d}, \mathrm{~J}=11.2 \mathrm{~Hz}, 1 \mathrm{H}), 1.68-1.63(\mathrm{~m}, 1 \mathrm{H}), 1.57(\mathrm{~d}, \mathrm{~J}$ $=6.8 \mathrm{~Hz}, 3 \mathrm{H}), 1.41-1.33(\mathrm{~m}, 1 \mathrm{H}), 1.29-1.26(\mathrm{~m}, 2 \mathrm{H}), 0.88(\mathrm{dd}, \mathrm{J}=9.0,4.3 \mathrm{~Hz}, 3 \mathrm{H}), 0.82(\mathrm{dd}, \mathrm{J}=$ $6.8,3.4 \mathrm{~Hz}, 1 \mathrm{H}), 0.49(\mathrm{td}, \mathrm{J}=14.0,6.8 \mathrm{~Hz}, 1 \mathrm{H})$.

${ }^{13} \mathrm{C}$ NMR $\left(151 \mathrm{MHz}, \mathrm{CDCl}_{3}\right): \delta 176.7,175.5,152.4,138.2,136.4,135.6,131.5,129.3,127.0,122.8$, $122.3,121.7,120.5,119.3,118.2,117.8,110.3,109.3,108.3,91.7,58.5,56.4,52.8,52.0,51.6,50.0$, $49.5,44.2,43.9,40.7,37.0,36.6,35.6,30.5,29.9,27.2,26.0,24.4,24.2,23.2,12.6,12.1$.

HRMS (ESI): Calculated for $\mathrm{C}_{42} \mathrm{H}_{50} \mathrm{~N}_{4} \mathrm{O}_{5}[\mathrm{M}+\mathrm{H}]^{+}: 691.3854$, found: 691.3854 .

$[\alpha]^{25} \mathrm{D}:+40^{\circ}\left(\mathrm{c}=1\right.$ in $\left.\mathrm{CHCl}_{3}\right)$.

TLC (ethyl acetate : methanol = $9: 1$ ) Rf: 0.4 (UV). 


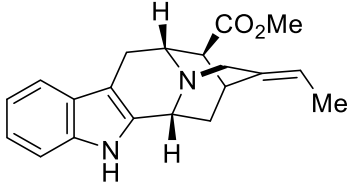

(-)-16-epi-pericyclivine (7)
1. $\mathrm{TMSCF}_{2} \mathrm{Br}, \mathrm{NH}_{4} \mathrm{OAC}$

2.

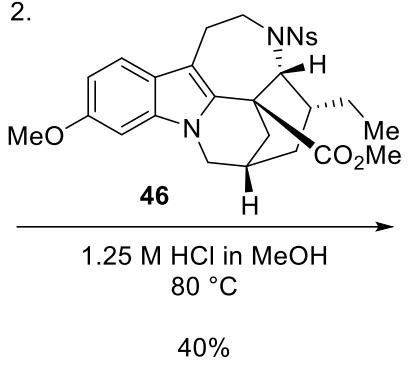

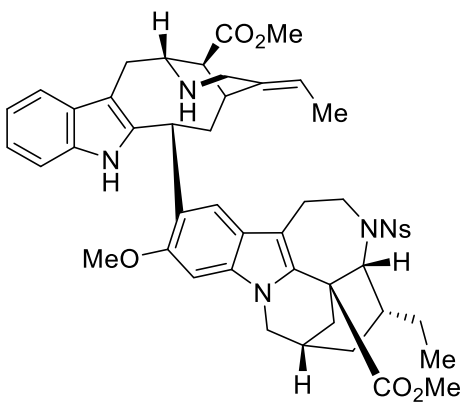

47

\section{heterodimer 47:}

To a stirred solution of 7 (36.1 $\mathrm{mg}, 0.112 \mathrm{mmol}, 2$ equiv) in 1,2-dichloroethane (1 mL) in seal tube was added $\mathrm{NH}_{4} \mathrm{OAc}$ ( $34.5 \mathrm{mg}, 0.448 \mathrm{mmol}, 8$ equiv) and $\mathrm{TMSCF}_{2} \mathrm{Br}(70 \mu \mathrm{L}, 0.448 \mathrm{mmol}, 8$ equiv) at room temperature. After 1.5 hours, $\mathrm{AgBF}_{4}(87.2 \mathrm{mg}, 0.448 \mathrm{mmol}, 8$ equiv) was added to the reaction mixture. After 1 hours, the resulting crude residue $\mathbf{4 3}$ was concentrated under reduced pressure.

To a solution of the crude mixture 43 in $1.25 \mathrm{M} \mathrm{HCl}$ in methanol $(1 \mathrm{~mL})$ was added 46 (31 mg, $0.056 \mathrm{mmol}, 1$ equiv) at room temperature and raised to $80^{\circ} \mathrm{C}$. After 12 hours, the resulting crude residue was dissolved in ethyl acetate $(5 \mathrm{~mL}$ ) and saturated aqueous sodium bicarbonate solution (10 $\mathrm{mL})$ and the layers were separated. The aqueous layer was extracted with ethyl acetate $(3 \times 10 \mathrm{~mL})$, and the combined organic layers were dried over anhydrous sodium sulfate and concentrated under reduced pressure. the reaction mixture was purified by column chromatography (silica gel: diam. $1 \mathrm{~cm}$, ht. $8 \mathrm{~cm}$; eluent : ethyl acetate $\left(1^{\mathrm{st}}\right)$, dichloromethane : methanol $\left.=100: 4\left(2^{\text {nd }}\right)\right)$ to afford $47(20 \mathrm{mg}$, $40 \%)$ as a yellow solid.

${ }^{1} \mathrm{H}$ NMR $\left(600 \mathrm{MHz}, \mathrm{CDCl}_{3}\right): \delta 8.08-8.03(\mathrm{~m}, 1 \mathrm{H}), 7.82(\mathrm{~s}, 1 \mathrm{H}), 7.66-7.63(\mathrm{~m}, 2 \mathrm{H}), 7.56(\mathrm{dt}, \mathrm{J}=17.2$, $4.9 \mathrm{~Hz}, 2 \mathrm{H}), 7.11-7.08(\mathrm{~m}, 1 \mathrm{H}), 7.06(\mathrm{dd}, \mathrm{J}=6.0,3.3 \mathrm{~Hz}, 2 \mathrm{H}), 6.94(\mathrm{~s}, 1 \mathrm{H}), 6.76(\mathrm{~s}, 1 \mathrm{H}), 5.36(\mathrm{q}, \mathrm{J}=$ $6.7 \mathrm{~Hz}, 1 \mathrm{H}), 4.88(\mathrm{~d}, \mathrm{~J}=11.9 \mathrm{~Hz}, 1 \mathrm{H}), 4.76(\mathrm{~d}, \mathrm{~J}=7.4 \mathrm{~Hz}, 1 \mathrm{H}), 4.17(\mathrm{dd}, \mathrm{J}=11.7,5.3 \mathrm{~Hz}, 1 \mathrm{H}), 4.06(\mathrm{~d}$, $\mathrm{J}=15.8 \mathrm{~Hz}, 1 \mathrm{H}), 4.03(\mathrm{~s}, 3 \mathrm{H}), 3.87(\mathrm{~s}, 1 \mathrm{H}), 3.84(\mathrm{t}, \mathrm{J}=9.7 \mathrm{~Hz}, 1 \mathrm{H}), 3.75(\mathrm{~d}, \mathrm{~J}=8.4 \mathrm{~Hz}, 2 \mathrm{H}), 3.65(\mathrm{t}, \mathrm{J}$ $=6.8 \mathrm{~Hz}, 1 \mathrm{H}), 3.61(\mathrm{~s}, 3 \mathrm{H}), 3.48(\mathrm{~s}, 3 \mathrm{H}), 3.42(\mathrm{~d}, \mathrm{~J}=6.9 \mathrm{~Hz}, 1 \mathrm{H}), 3.32-3.24(\mathrm{~m}, 1 \mathrm{H}), 3.12(\mathrm{~d}, \mathrm{~J}=15.2$ $\mathrm{Hz}, 1 \mathrm{H}), 3.04(\mathrm{~s}, 1 \mathrm{H}), 2.94(\mathrm{q}, \mathrm{J}=12.7 \mathrm{~Hz}, 1 \mathrm{H}), 2.78(\mathrm{~s}, 2 \mathrm{H}), 2.50(\mathrm{~s}, 2 \mathrm{H}), 2.32-2.25(\mathrm{~m}, 1 \mathrm{H}), 2.23-$ $2.10(\mathrm{~m}, 2 \mathrm{H}), 1.95$ (ddd, J = 25.3, $16.1,7.0 \mathrm{~Hz}, 2 \mathrm{H}), 1.60(\mathrm{~d}, \mathrm{~J}=6.6 \mathrm{~Hz}, 3 \mathrm{H}), 1.54(\mathrm{~d}, \mathrm{~J}=13.0 \mathrm{~Hz}, 1 \mathrm{H})$, $0.98(\mathrm{~s}, 1 \mathrm{H}), 0.53(\mathrm{t}, \mathrm{J}=7.4 \mathrm{~Hz}, 3 \mathrm{H}),-0.57(\mathrm{dq}, \mathrm{J}=13.2,7.2 \mathrm{~Hz}, 1 \mathrm{H})$.

${ }^{13} \mathrm{C}$ NMR $\left(151 \mathrm{MHz}, \mathrm{CDCl}_{3}\right): \delta 175.6,173.9,152.8,148.8,138.0,136.8,136.5,135.6,133.6,133.4$, 132.2, 131.5, 131.3, 129.3, 127.6, 124.0, 121.7, 121.6, 119.8, 119.2, 118.6, 117.8, 112.0, 110.3, 109.9, $91.9,61.1,56.1,52.8,51.9,51.7,48.7,47.4,46.8,44.6,44.4,40.4,37.6,36.9,36.7,35.4,31.4,26.9$, 24.8, 24.4, 21.3, 13.9, 12.1.

HRMS (ESI): Calculated for $\mathrm{C}_{48} \mathrm{H}_{53} \mathrm{~N}_{5} \mathrm{O}_{9} \mathrm{~S}[\mathrm{M}+\mathrm{H}]^{+}: 876.3637$, found: 876.3639 .

$[\alpha]^{25} \mathrm{D}:+76^{\circ}\left(c=1\right.$ in $\left.\mathrm{CHCl}_{3}\right)$.

TLC (ethyl acetate : methanol = $9: 1$ ) Rf: 0.5 (UV). 


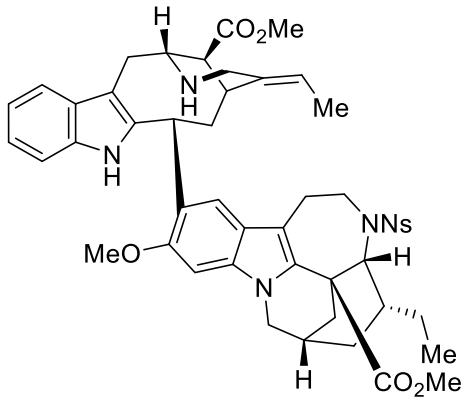

47

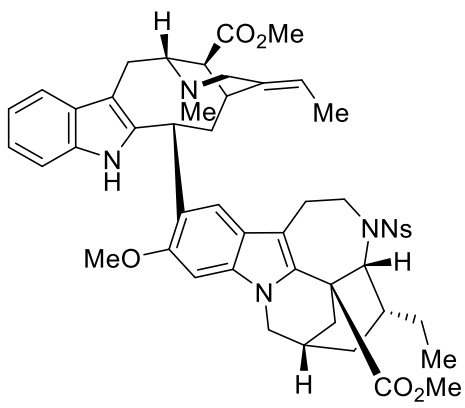

S17

\section{tertiary amine S17:}

To a solution of $47(20 \mathrm{mg}, 0.0229 \mathrm{mmol}, 1$ equiv) in ethyl acetate $(2 \mathrm{~mL})$ and methanol $(0.2$ $\mathrm{mL}$ ) was added formaldehyde solution (in $\mathrm{H}_{2} \mathrm{O} 37$ wt. \%) (19 $\mu \mathrm{L}, 0.2285 \mathrm{mmol}, 10$ equiv) at room temperature. After 20 minutes, to a solution the reaction mixture was added $\mathrm{NaBH}(\mathrm{OAc})_{3}(72.6 \mathrm{mg}$, $0.3427 \mathrm{mmol}, 15$ equiv). After 1.5 hours, the resulting crude residue was dissolved in ethyl acetate (5 $\mathrm{mL}$ ) and saturated aqueous sodium bicarbonate solution $(10 \mathrm{~mL})$ and the layers were separated. The aqueous layer was extracted with ethyl acetate $(3 \times 10 \mathrm{~mL})$, and the combined organic layers were dried over anhydrous sodium sulfate and concentrated under reduced pressure. the reaction mixture was purified by column chromatography (silica gel: diam. $1 \mathrm{~cm}$, ht. $8 \mathrm{~cm}$; eluent : ethyl acetate) to afford S17 (13.7 $\mathrm{mg}, 67 \%)$ as a yellow solid.

${ }^{1} \mathrm{H}$ NMR $\left(600 \mathrm{MHz}, \mathrm{CDCl}_{3}\right)$ : $\delta 8.08-8.05(\mathrm{~m}, 1 \mathrm{H}), 7.72(\mathrm{~s}, 1 \mathrm{H}), 7.67-7.63(\mathrm{~m}, 2 \mathrm{H}), 7.60-7.54(\mathrm{~m}$, $2 \mathrm{H}), 7.11-7.05(\mathrm{~m}, 3 \mathrm{H}), 6.90(\mathrm{~s}, 1 \mathrm{H}), 6.75(\mathrm{~s}, 1 \mathrm{H}), 5.42(\mathrm{~d}, \mathrm{~J}=7.1 \mathrm{~Hz}, 1 \mathrm{H}), 4.78(\mathrm{~d}, \mathrm{~J}=12.4 \mathrm{~Hz}, 1 \mathrm{H})$, $4.75(\mathrm{~d}, \mathrm{~J}=7.5 \mathrm{~Hz}, 1 \mathrm{H}), 4.17(\mathrm{dd}, \mathrm{J}=11.7,5.3 \mathrm{~Hz}, 1 \mathrm{H}), 4.01(\mathrm{~s}, 3 \mathrm{H}), 3.90-3.81(\mathrm{~m}, 3 \mathrm{H}), 3.70-3.62$ $(\mathrm{m}, 2 \mathrm{H}), 3.58(\mathrm{~s}, 3 \mathrm{H}), 3.47(\mathrm{~s}, 3 \mathrm{H}), 3.30-3.22(\mathrm{~m}, 2 \mathrm{H}), 3.10(\mathrm{~s}, 1 \mathrm{H}), 2.96(\mathrm{~d}, \mathrm{~J}=13.2 \mathrm{~Hz}, 1 \mathrm{H}), 2.79-$ $2.72(\mathrm{~m}, 2 \mathrm{H}), 2.55(\mathrm{~s}, 3 \mathrm{H}), 2.50(\mathrm{~s}, 1 \mathrm{H}), 2.30-2.24(\mathrm{~m}, 1 \mathrm{H}), 2.20(\mathrm{~d}, \mathrm{~J}=12.5 \mathrm{~Hz}, 1 \mathrm{H}), 2.13(\mathrm{dd}, \mathrm{J}=$ 12.8, 4.3 Hz, 1H), $1.96(\mathrm{dt}, \mathrm{J}=14.0,6.7 \mathrm{~Hz}, 1 \mathrm{H}), 1.90(\mathrm{dd}, \mathrm{J}=14.6,7.3 \mathrm{~Hz}, 1 \mathrm{H}), 1.71(\mathrm{~d}, \mathrm{~J}=6.6 \mathrm{~Hz}$, $3 \mathrm{H}), 1.53(\mathrm{~d}, \mathrm{~J}=15.0 \mathrm{~Hz}, 1 \mathrm{H}), 1.28(\mathrm{~d}, \mathrm{~J}=9.0 \mathrm{~Hz}, 1 \mathrm{H}), 0.99(\mathrm{t}, \mathrm{J}=11.5 \mathrm{~Hz}, 1 \mathrm{H}), 0.90-0.84(\mathrm{~m}, 1 \mathrm{H})$, $0.54(\mathrm{t}, \mathrm{J}=7.3 \mathrm{~Hz}, 3 \mathrm{H}),-0.55(\mathrm{tt}, \mathrm{J}=12.9,7.0 \mathrm{~Hz}, 1 \mathrm{H})$.

${ }^{13}$ C NMR $\left(101 \mathrm{MHz}, \mathrm{CDCl}_{3}\right): \delta$ 175.2, 174.0, 152.9, 148.8, 138.1, 136.5, 136.0, 135.5, 133.7, 133.5, $132.0,131.5,131.4,129.5,127.8,124.0,121.5,121.5,119.1,118.6,118.6,117.7,111.9,110.4,110.1$, $91.9,61.1,59.3,56.1,52.8,52.6,51.9,48.7,47.4,46.7,44.4,42.7,40.4,37.3,36.9,35.5,34.1,31.4$, $29.9,26.9,24.4,21.3,14.0,12.5$.

HRMS (ESI): Calculated for $\mathrm{C}_{49} \mathrm{H}_{55} \mathrm{~N}_{5} \mathrm{O}_{9} \mathrm{~S}[\mathrm{M}+\mathrm{H}]+:$ 890.3793, found: 890.3791 .

$[\alpha]^{25} \mathrm{D}:+60^{\circ}\left(c=1\right.$ in $\left.\mathrm{CHCl}_{3}\right)$.

TLC (ethyl acetate : methanol = $9: 1$ ) Rf: 0.7 (UV). 


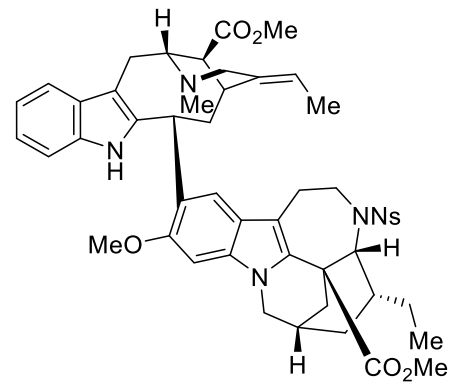

S17

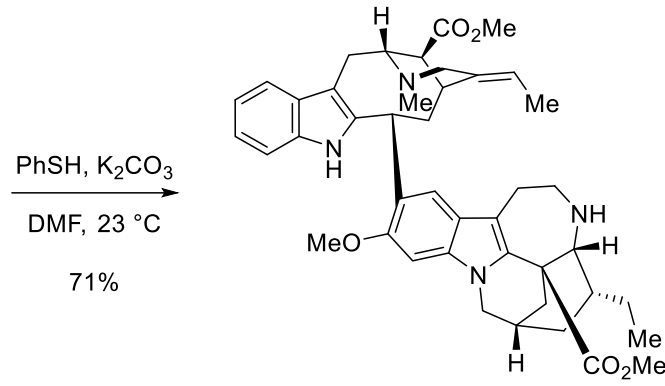

(+)-16-dehydroxymethyl-tabercarpamine A (9)

\section{(+)-16-dehydroxymethyl-tabercarpamine A (9):}

To a solution of $\mathbf{S} 16$ (13 mg, $0.0146 \mathrm{mmol}, 1$ equiv) in DMF $(1 \mathrm{~mL})$ was added $\mathrm{K}_{2} \mathrm{CO}_{3}(40.4$ $\mathrm{mg}, 0.2921 \mathrm{mmol}, 20$ equiv) and $\mathrm{PhSH}(15 \mu \mathrm{L}, 0.1461 \mathrm{mmol}, 10$ equiv) at room temperature. After 5 hours, the resulting reaction mixture was diluted with ethyl acetate $(5 \mathrm{~mL})$ and saturated aqueous sodium chloride solution $(10 \mathrm{~mL})$ and the layers were separated. The organic layer was washed with brine $(3 \times 10 \mathrm{~mL})$, and the combined organic layers were dried over anhydrous sodium sulfate and concentrated under reduced pressure. The resulting crude residue was purified by column chromatography (silica gel: diam. $2 \mathrm{~cm}$, ht. $9 \mathrm{~cm}$; eluent : ethyl acetate) to afford $\mathbf{9}(7.3 \mathrm{mg}, 71 \%)$ as a yellow solid.

${ }^{1} \mathrm{H}$ NMR $\left(600 \mathrm{MHz}, \mathrm{CDCl}_{3}\right): \delta 7.82(\mathrm{~s}, 1 \mathrm{H}), 7.59-7.55(\mathrm{~m}, 1 \mathrm{H}), 7.13-7.09(\mathrm{~m}, 1 \mathrm{H}), 7.09-7.05(\mathrm{~m}$, $2 \mathrm{H}), 6.98(\mathrm{~s}, 1 \mathrm{H}), 6.80(\mathrm{~s}, 1 \mathrm{H}), 5.39(\mathrm{q}, \mathrm{J}=6.8 \mathrm{~Hz}, 1 \mathrm{H}), 4.80(\mathrm{~d}, \mathrm{~J}=11.9 \mathrm{~Hz}, 1 \mathrm{H}), 4.06(\mathrm{dd}, \mathrm{J}=11.2$, $3.3 \mathrm{~Hz}, 1 \mathrm{H}), 4.02(\mathrm{~s}, 3 \mathrm{H}), 3.89-3.80(\mathrm{~m}, 3 \mathrm{H}), 3.77-3.67(\mathrm{~m}, 1 \mathrm{H}), 3.62(\mathrm{~s}, 3 \mathrm{H}), 3.58(\mathrm{~s}, 3 \mathrm{H}), 3.28-$ $3.20(\mathrm{~m}, 1 \mathrm{H}), 3.18-3.05(\mathrm{~m}, 2 \mathrm{H}), 2.94(\mathrm{~d}, \mathrm{~J}=13.4 \mathrm{~Hz}, 1 \mathrm{H}), 2.81-2.71(\mathrm{~m}, 2 \mathrm{H}), 2.60-2.55(\mathrm{~m}, 2 \mathrm{H})$, $2.53(\mathrm{~s}, 3 \mathrm{H}), 2.48-2.41(\mathrm{~m}, 1 \mathrm{H}), 2.21(\mathrm{dd}, \mathrm{J}=13.1,5.6 \mathrm{~Hz}, 1 \mathrm{H}), 1.92(\mathrm{dd}, \mathrm{J}=15.0,7.6 \mathrm{~Hz}, 1 \mathrm{H}), 1.87$ $(\mathrm{d}, \mathrm{J}=12.9 \mathrm{~Hz}, 1 \mathrm{H}), 1.81-1.73(\mathrm{~m}, 1 \mathrm{H}), 1.67(\mathrm{~d}, \mathrm{~J}=6.5 \mathrm{~Hz}, 3 \mathrm{H}), 1.63(\mathrm{~d}, \mathrm{~J}=13.7 \mathrm{~Hz}, 1 \mathrm{H}), 1,58-$ $1.55(\mathrm{~m}, 1 \mathrm{H}), 1.36(\mathrm{dt}, \mathrm{J}=14.4,6.9 \mathrm{~Hz}, 1 \mathrm{H}), 1.28(\mathrm{~d}, \mathrm{~J}=9.3 \mathrm{~Hz}, 1 \mathrm{H}), 0.96(\mathrm{dq}, \mathrm{J}=15.4,7.4,7.0 \mathrm{~Hz}$, $1 \mathrm{H}), 0.89(\mathrm{t}, \mathrm{J}=7.3 \mathrm{~Hz}, 3 \mathrm{H}), 0.81(\mathrm{dt}, \mathrm{J}=15.5,6.4 \mathrm{~Hz}, 1 \mathrm{H}), 0.49(\mathrm{td}, \mathrm{J}=14.2,6.7 \mathrm{~Hz}, 1 \mathrm{H})$.

${ }^{13} \mathrm{C}$ NMR $\left(151 \mathrm{MHz}, \mathrm{CDCl}_{3}\right): \delta 176.7,175.3,152.5,138.3,136.4,135.8,135.6,131.4,129.6,127.4$, 122.7, 121.5, 119.9, 119.0, 118.0, 117.6, 110.4, 109.9, 108.2, 91.8, 59.4, 58.5, 56.4, 52.8, 52.6, 51.9, 50.0, 49.6, 44.4, 42.7, 40.8, 37.2, 37.0, 35.8, 34.1, 30.5, 27.2, 26.0, 24.3, 23.2, 17.4, 12.6, 12.4.

HRMS (ESI): Calculated for $\mathrm{C}_{43} \mathrm{H}_{52} \mathrm{~N}_{4} \mathrm{O}_{5}[\mathrm{M}+\mathrm{H}]^{+}:$705.4010, found: 705.4016 .

$[\alpha]^{25} \mathrm{D}:+39^{\circ}\left(\mathrm{c}=0.9\right.$ in $\left.\mathrm{CHCl}_{3}\right)$.

TLC (ethyl acetate : methanol = $9: 1$ ) Rf: 0.3 (UV). 


\section{Comparison of Spectroscopic Data of Natural and Synthetic Compounds}

4.1 Comparison of Spectroscopic Data of Natural (-)-secu'amamine E (2) and Our Synthetic Sample of $(-)-2$

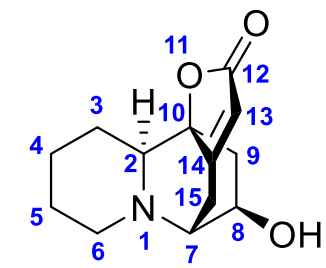

(-)-secu'amamine E (2)

Table S1. Comparison of ${ }^{1} \mathrm{H}$ NMR spectroscopic data of natural (-)-secu'amamine E (2) and our synthetic sample of $(-)-2$

\begin{tabular}{cccc}
\hline position & $\begin{array}{c}\text { natural 2 } \\
\text { (3) }\end{array}$ & $\begin{array}{c}\text { synthetic } 2 \\
\delta_{1}(\mathrm{ppm} ; \text { multi, } J \text { in Hz) }\end{array}$ & $\begin{array}{c}\text { deviation } \\
\Delta \delta=\delta_{1}-\delta_{2} \\
(\mathrm{ppm})\end{array}$ \\
\hline 2 & $2.79(\mathrm{dd}, 12.0,1.8)$ & $2.78(\mathrm{dd}, 4.1,1.8)$ & 0.01 \\
$3 \alpha$ & $1.50(\mathrm{~m})$ & $1.45-1.51(\mathrm{~m})$ & 0.02 \\
$3 \beta$ & $0.86(\mathrm{qd}, 12.0,4.0)$ & $0.87(\mathrm{qd}, 12.0,4.1)$ & -0.01 \\
$4 a$ & $1.35(\mathrm{~m})$ & $1.28-1.35(\mathrm{~m})$ & 0.03 \\
$4 \beta$ & $1.81(\mathrm{~m})$ & $1.76-1.86(\mathrm{~m})$ & 0 \\
$5 \mathrm{a}$ & $1.53(\mathrm{~m})$ & $1.52-1.56(\mathrm{~m})$ & -0.01 \\
$5 \mathrm{~b}$ & $1.58(\mathrm{~m})$ & $1.56-1.62(\mathrm{~m})$ & -0.01 \\
$6 \mathrm{a}$ & $2.75(\mathrm{td}, 11.0,3.2)$ & $2.74(\mathrm{dd}, 10.8,3.3)$ & 0.01 \\
$6 \beta$ & $2.94(\mathrm{~m})$ & $2.92-2.95(\mathrm{~m})$ & 0 \\
7 & $2.88(\mathrm{ddd}, 5.7,1.4,1.3)$ & $2.89(\mathrm{td}, 3.5,1.3)$ & -0.01 \\
8 & $4.30(\mathrm{dddd}, 9.5,5.0,3.0,1.4)$ & $4.30(\mathrm{dddd}, 9.6,4.7,3.1,1.4)$ & 0 \\
$9 \mathrm{a}$ & $2.66(\mathrm{dd}, 12.2,9.5)$ & $2.67(\mathrm{dd}, 12.2,9.6)$ & -0.01 \\
$9 \mathrm{~b}$ & $1.38(\mathrm{dd}, 12.2,5.0)$ & $1.38(\mathrm{dd}, 12.2,4.9)$ & 0 \\
13 & $5.74(\mathrm{t}, 1.9)$ & $5.74(\mathrm{t}, 2.0)$ & 0 \\
$15 \mathrm{a}$ & $2.82(\mathrm{~m})$ & $2.80-2.84(\mathrm{~m})$ & 0 \\
$15 \mathrm{~b}$ & $2.97(\mathrm{ddd}, 18.4,1.9,1.3)$ & $2.95-3.01(\mathrm{~m})$ & -0.01
\end{tabular}


Table S2. Comparison of ${ }^{13} \mathrm{C}$ NMR spectroscopic data of natural (-)-secu'amamine $\mathrm{E}(2)$ and our synthetic sample of $(-)-2$

\begin{tabular}{cccc}
\hline position & $\begin{array}{c}\text { natural } \mathbf{2}^{3} \\
\delta_{1}(\mathrm{ppm})\end{array}$ & $\begin{array}{c}\text { synthetic 2 } \\
\delta_{2}(\mathrm{ppm})\end{array}$ & $\begin{array}{c}\text { deviation } \\
\Delta \delta=\delta_{1}-\delta_{2} \\
(\mathrm{ppm})\end{array}$ \\
\hline 2 & 66.5 & 66.4 & 0.1 \\
3 & 26.8 & 26.7 & 0.1 \\
4 & 25.1 & 25.1 & 0 \\
5 & 27.7 & 27.7 & 0 \\
6 & 53.6 & 53.5 & 0.1 \\
7 & 60.4 & 60.3 & 0.1 \\
8 & 65.4 & 65.3 & 0.1 \\
9 & 41.6 & 41.5 & 0.1 \\
10 & 86.3 & 86.2 & 0.1 \\
12 & 176.3 & 176.3 & 0 \\
13 & 112.0 & 112.0 & 0 \\
14 & 177.3 & 117.2 & 0.1 \\
15 & 30.3 & 30.3 & 0
\end{tabular}


4.2 Comparison of Spectroscopic Data of Natural (-)-3-hydroxy-3,4-secocoronaridine (33) and Our Synthetic (+)-3-hydroxy-3,4-secocoronaridine (33)

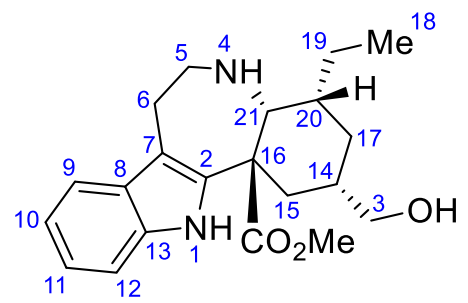

(+)-3-hydroxy-3,4-secocoronaridine (33)

Table S3. Comparison of ${ }^{1} \mathrm{H}$ NMR spectroscopic data of natural (-)-3-hydroxy-3,4secocoronaridine (33) and our synthetic (+)-3-hydroxy-3,4-secocoronaridine (33)

\begin{tabular}{|c|c|c|c|}
\hline position & $\begin{array}{c}\text { natural } 33^{6} \\
\delta_{1}(\mathrm{ppm} ; \text { multi, } J \text { in } \mathrm{Hz})\end{array}$ & $\begin{array}{c}\text { synthetic } 33 \\
\delta_{2}(\mathrm{ppm} ; \text { multi, } J \text { in } \mathrm{Hz})\end{array}$ & $\begin{array}{c}\text { deviation } \\
\Delta \delta=\delta_{1}-\delta_{2} \\
\text { (ppm) }\end{array}$ \\
\hline 3 & $3.48(d, 5.9)$ & $3.45(d, 5.9)$ & 0.03 \\
\hline $5 a$ & $2.85-2.90(\mathrm{~m})$ & $2.86-2.97(\mathrm{~m})$ & -0.01 \\
\hline $5 b$ & $3.31-3.42(\mathrm{~m})$ & $3.33-3.41(\mathrm{~m})$ & -0.02 \\
\hline 6 & $3.08-3.20(\mathrm{~m})$ & $3.08-3.18(\mathrm{~m})$ & 0 \\
\hline 9 & $7.49(\mathrm{br} \mathrm{d}, 7.6)$ & $7.46(d, 7.8)$ & 0.03 \\
\hline 10 & $7.08(\mathrm{td}, 7.6,1.1)$ & $7.05(t, 7.4)$ & 0.03 \\
\hline 11 & $7.14(\mathrm{td}, 7.6,1.1)$ & $7.12(t, 7.1)$ & 0.02 \\
\hline 12 & $7.25(\mathrm{br} \mathrm{d}, 7.6)$ & $7.23(d, 8.0)$ & 0.02 \\
\hline 14 & $1.72-1.90(\mathrm{~m})$ & $1.70-1.80(\mathrm{~m})$ & 0.02 \\
\hline $15 a$ & $0.95-1.05(\mathrm{~m})$ & $0.95-1.05(\mathrm{~m})$ & 0 \\
\hline $15 b$ & $1.35-1.59(\mathrm{~m})$ & $1.32-1.57(\mathrm{~m})$ & 0.03 \\
\hline $17 a$ & $1.60(t, 13.1)$ & $1.63(t, 12.9)$ & -0.03 \\
\hline $17 \mathrm{~b}$ & $2.48(\mathrm{br} \mathrm{d}, 13.1)$ & $2.47(\mathrm{br} \mathrm{d}, 13.0)$ & 0.01 \\
\hline 18 & $0.95(t, 7.2)$ & $0.96(t, 7.3)$ & -0.01 \\
\hline 19 & $1.35-1.59(\mathrm{~m})$ & $1.32-1.57(\mathrm{~m})$ & 0.03 \\
\hline 20 & $1.35-1.59(\mathrm{~m})$ & $1.32-1.57(\mathrm{~m})$ & 0.03 \\
\hline 21 & $3.85(d, 1.7)$ & $3.87(d, 2.4)$ & -0.02 \\
\hline $\mathrm{CO}_{2} \mathrm{Me}$ & $3.74(\mathrm{~s})$ & $3.71(\mathrm{~s})$ & 0.03 \\
\hline $\mathrm{N}(1) \mathrm{H}$ & 8.11 (br s) & 8.11 (br s) & 0 \\
\hline
\end{tabular}


Table S4. Comparison of ${ }^{13} \mathrm{C}$ NMR spectroscopic data of natural (-)-3-hydroxy-3,4secocoronaridine (33) and synthetic (+)-3-hydroxy-3,4-secocoronaridine (33)

\begin{tabular}{|c|c|c|c|}
\hline position & $\begin{array}{c}\text { natural } \mathbf{3 3}^{6} \\
\delta_{1}(\mathrm{ppm})\end{array}$ & $\begin{array}{c}\text { synthetic } \mathbf{3 3} \\
\delta_{2}(\mathrm{ppm})\end{array}$ & $\begin{array}{c}\text { deviation } \\
\Delta \delta=\delta_{1}-\delta_{2} \\
(\mathrm{ppm})\end{array}$ \\
\hline 2 & 135.2 & 135.4 & -0.2 \\
\hline 3 & 67.7 & 68.0 & -0.3 \\
\hline 5 & 49.2 & 49.5 & -0.3 \\
\hline 6 & 24.7 & 25.1 & -0.4 \\
\hline 7 & * & 110.7 & \\
\hline 8 & 128.4 & 128.7 & -0.3 \\
\hline 9 & 118.2 & 118.5 & -0.3 \\
\hline 10 & 119.2 & 119.5 & -0.3 \\
\hline 11 & 121.9 & 122.2 & -0.3 \\
\hline 12 & 110.5 & 110.7 & -0.2 \\
\hline 13 & 134.2 & 134.4 & -0.2 \\
\hline 14 & 37.2 & 37.5 & -0.3 \\
\hline 15 & 28.6 & 28.9 & -0.3 \\
\hline 16 & 56.2 & 56.5 & -0.3 \\
\hline 17 & 33.6 & 33.9 & -0.3 \\
\hline 18 & 11.7 & 12.0 & -0.3 \\
\hline 19 & 25.8 & 26.1 & -0.3 \\
\hline 20 & 40.9 & 41.2 & -0.3 \\
\hline 21 & 57.7 & 58.2 & -0.5 \\
\hline$\underline{\mathrm{CO}_{2} \mathrm{Me}}$ & 174.2 & 174.4 & -0.2 \\
\hline $\mathrm{CO}_{2} \underline{\mathrm{Me}}$ & 52.6 & 52.8 & -0.2 \\
\hline
\end{tabular}


4.3 Comparison of Spectroscopic Data of Natural (-)-3-oxocoronaridine (35) and Synthetic (+)3-oxocoronaridine (35)

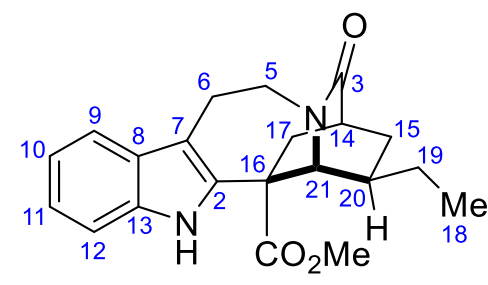

(+)-3-oxocoronaridine (35)

Table S5. Comparison of ${ }^{1} \mathrm{H}$ NMR spectroscopic data of natural (-)-3-oxocoronaridine (35) and synthetic (+)-3-oxocoronaridine (35)

\begin{tabular}{cccc}
\hline position & $\begin{array}{c}\text { natural 35 } \\
\delta_{1}(\mathrm{ppm} ; \text { multi, } J \text { in Hz) }\end{array}$ & $\begin{array}{c}\text { synthetic } 35 \\
\delta_{2}(\mathrm{ppm} ; \text { multi, } J \text { in } \mathrm{Hz})\end{array}$ & $\begin{array}{c}\text { deviation } \\
\Delta \delta=\delta_{1}-\delta_{2} \\
(\mathrm{ppm})\end{array}$ \\
\hline $5 \alpha$ & $4.46-4.50(\mathrm{~m})$ & $4.46(\mathrm{q}, 5.4,4.4)$ & 0 \\
$5 \beta$ & $3.20(\mathrm{~m})$ & $3.15-3.25(\mathrm{~m})$ & 0.05 \\
$6 \alpha$ & $3.13-3.19(\mathrm{~m})$ & $3.15-3.25(\mathrm{~m})$ & -0.02 \\
$6 \beta$ & $3.21-3.23(\mathrm{~m})$ & $3.15-3.25(\mathrm{~m})$ & 0.02 \\
9 & $7.48(\mathrm{br} \mathrm{d}, 7.3)$ & $7.46(\mathrm{br} \mathrm{d}, 7.8)$ & 0.02 \\
10 & $7.10(\mathrm{ddd}, 7.1,7.1,1.0)$ & $7.07(\mathrm{td}, 7.4,1.2)$ & 0.03 \\
11 & $7.15(\mathrm{ddd}, 7.17 .1,1.2)$ & $7.13(\mathrm{ddd}, 8.0,7.0,1.3)$ & 0.02 \\
12 & $7.25(\mathrm{br} \mathrm{d}, 6.4)$ & $7.22(\mathrm{br} \mathrm{d}, 8.0)$ & 0.03 \\
14 & $2.62(\mathrm{br} \mathrm{s})$ & $2.56-2.68(\mathrm{~m})$ & 0.01 \\
$15 \alpha$ & $2.00(\mathrm{ddd}, 13.2,10.0,3.2)$ & $1.98(\mathrm{ddd}, 13.3,10.0,3.3)$ & 0.02 \\
$15 \beta$ & $1.38-1.42(\mathrm{~m})$ & $1.35-1.40(\mathrm{~m})$ & 0.03 \\
$17 \alpha$ & $2.66(\mathrm{~d}, 1.7)$ & $2.56-2.68(\mathrm{~m})$ & 0.04 \\
$17 \beta$ & $2.30-2.32(\mathrm{~m})$ & $2.29(\mathrm{ddd}, 13.3,4.0,2.5)$ & 0.02 \\
18 & $0.98(\mathrm{t}, 7.5)$ & $0.96(\mathrm{t}, 7.4)$ & 0.02 \\
$19 \alpha$ & $1.52-1.56(\mathrm{~m})$ & $1.51(\mathrm{dq}, 14.6,7.3)$ & 0.02 \\
$19 \beta$ & $1.40-1.43(\mathrm{~m})$ & $1.40-1.45(\mathrm{~m})$ & 0.01 \\
20 & $1.72-1.78(\mathrm{~m})$ & $1.69-1.78(\mathrm{~m})$ & 0.02 \\
21 & $4.51(\mathrm{br} \mathrm{s})$ & $4.50(\mathrm{br} \mathrm{s})$ & 0.01 \\
$\mathrm{NH}$ & $7.96(\mathrm{br} \mathrm{s})$ & $7.97(\mathrm{br} \mathrm{s})$ & -0.01 \\
$\mathrm{CO}{ }_{2} \mathrm{Me}$ & $3.87(\mathrm{~s})$ & $3.71(\mathrm{~s})$ & 0.16
\end{tabular}


Table S6. Comparison of ${ }^{13} \mathrm{C}$ NMR spectroscopic data of natural (-)-3-oxocoronaridine (35) and synthetic (+)-3-oxocoronaridine (35)

\begin{tabular}{cccc}
\hline position & $\begin{array}{c}\text { natural } 35^{8} \\
\delta_{1}(\mathrm{ppm})\end{array}$ & $\begin{array}{c}\text { synthetic } 35 \\
\delta_{2}(\mathrm{ppm})\end{array}$ & $\begin{array}{c}\text { deviation } \\
\Delta \delta=\delta_{1}-\delta_{2} \\
(\mathrm{ppm})\end{array}$ \\
\hline 2 & 133.8 & 134.1 & -0.3 \\
3 & 175.7 & 175.9 & -0.2 \\
5 & 42.7 & 42.9 & -0.2 \\
6 & 21.0 & 21.3 & -0.3 \\
7 & 109.7 & 109.6 & 0.1 \\
8 & 127.8 & 128.0 & -0.2 \\
9 & 118.4 & 118.6 & -0.2 \\
10 & 119.6 & 119.8 & -0.2 \\
11 & 122.4 & 122.6 & -0.2 \\
12 & 110.5 & 110.8 & -0.3 \\
13 & 135.6 & 135.9 & -0.3 \\
14 & 38.1 & 38.4 & -0.3 \\
15 & 30.9 & 31.2 & -0.3 \\
16 & 55.5 & 55.7 & -0.2 \\
17 & 35.9 & 36.1 & -0.2 \\
18 & 11.3 & 11.6 & -0.3 \\
19 & 27.6 & 27.8 & -0.2 \\
20 & 35.4 & 35.7 & -0.3 \\
21 & 56.1 & 56.3 & -0.2 \\
$\mathrm{CO}_{2} \mathrm{Me}$ & 53.0 & 53.2 & -0.2 \\
$\mathrm{CO}_{2} \mathrm{Me}$ & 173.0 & 173.3 & -0.3 \\
& & &
\end{tabular}


4.4 Comparison of Spectroscopic Data of Natural (-)-10,11-demethoxychippiine (4) and Our Synthetic (+)-10,11-demethoxychippiine (4)

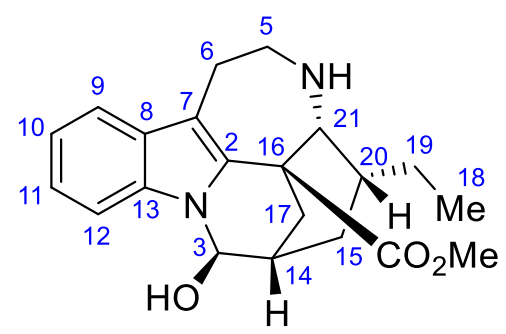

(+)-10,11-demethoxychippiine (4)

Table S7. Comparison of ${ }^{1} \mathrm{H}$ NMR spectroscopic data of natural (-)-10,11-demethoxychippiine (4) and our synthetic (+)-10,11-demethoxychippiine (4)

\begin{tabular}{cccc}
\hline position & $\begin{array}{c}\text { natural } \mathbf{4}^{9} \\
\delta_{1}(\mathrm{ppm} ; \text { multi, } J \text { in Hz) }\end{array}$ & $\begin{array}{c}\text { synthetic } 4 \\
\delta_{2}(\mathrm{ppm} ; \text { multi, } J \text { in } \mathrm{Hz})\end{array}$ & $\begin{array}{c}\text { deviation } \\
\Delta \delta=\delta_{1}-\delta_{2} \\
(\mathrm{ppm})\end{array}$ \\
\hline 3 & $5.46(\mathrm{~d}, 2.2)$ & $5.43(\mathrm{~d}, 2.3)$ & 0.03 \\
$5 \mathrm{a}$ & $3.19(\mathrm{~m})$ & $3.09-3.20(\mathrm{~m})$ & 0.04 \\
$5 \mathrm{~b}$ & $2.95(\mathrm{~m})$ & $2.85-2.99(\mathrm{~m})$ & 0.03 \\
$6 \mathrm{a}$ & $2.67(\mathrm{~m})$ & $2.59-2.74(\mathrm{~m})$ & 0 \\
$6 \mathrm{~b}$ & $2.67(\mathrm{~m})$ & $2.59-2.74(\mathrm{~m})$ & 0 \\
9 & $7.50(\mathrm{dd}, 7.1,1.1)$ & $7.48(\mathrm{~d}, 7.7)$ & 0.02 \\
10 & $7.16(\mathrm{ddd}, 7.1,7.1,1.1)$ & $7.15(\mathrm{td}, 7.5,1.1)$ & 0.01 \\
11 & $7.22(\mathrm{ddd}, 7.1,7.1,1.1)$ & $7.20(\mathrm{ddd}, 8.1,7.1,1.3)$ & 0.02 \\
12 & $7.47(\mathrm{dd}, 7.1,1.1)$ & $7.43(\mathrm{~d}, 8.0)$ & 0.04 \\
14 & $2.49(\mathrm{~m})$ & $2.41-2.52(\mathrm{~m})$ & 0.03 \\
$15 \mathrm{a}$ & $0.32(\mathrm{ddd}, 14.2,14.1,7.1)$ & $0.30(\mathrm{td}, 14.2,7.0)$ & 0.02 \\
$15 \mathrm{~b}$ & $1.62(\mathrm{ddd}, 14.2,10.5,3.4)$ & $1.60(\mathrm{ddd}, 14.1,10.4,3.4)$ & 0.02 \\
$17 \mathrm{a}$ & $2.34(\mathrm{dd}, 13.4,1.0)$ & $2.31(\mathrm{dd}, 13.5,1.4)$ & 0.03 \\
$17 \mathrm{~b}$ & $2.02(\mathrm{dd}, 13.4,5.0)$ & $2.00(\mathrm{dd}, 13.5,5.3)$ & 0.02 \\
18 & $0.91(\mathrm{t}, 7.0)$ & $0.89(\mathrm{t}, 7.2)$ & 0.02 \\
$19 \mathrm{a}$ & $1.00(\mathrm{dqd}, 12.9,6.9,7.1)$ & $0.97(\mathrm{tt}, 13.1,6.0)$ & 0.03 \\
$19 \mathrm{~b}$ & $1.39(\mathrm{dqd}, 12.9,6.9,7.1)$ & $1.30-1.41(\mathrm{~m})$ & 0.03 \\
20 & $1.84(\mathrm{ddddd}, 14.0,6.9,6.9$, & $1.81(\mathrm{dqd}, 14.2,7.1,3.4)$ & 0.03 \\
21 & $6.9,3.4)$ & $3.90(\mathrm{~d}, 7.0)$ & 0.03 \\
$\mathrm{CO}_{2} \mathrm{Me}$ & $3.93(\mathrm{~d}, 6.9)$ & $3.68(\mathrm{~s})$ & 0.02
\end{tabular}


Table S8. Comparison of ${ }^{13} \mathrm{C}$ NMR spectroscopic data of natural (-)-10,11-demethoxychippiine (4) and our synthetic (+)-10,11-demethoxychippiine (4)

\begin{tabular}{cccc}
\hline position & $\begin{array}{c}\text { natural } 4^{9} \\
\delta_{1}(\mathrm{ppm})\end{array}$ & $\begin{array}{c}\text { synthetic } 4 \\
\delta_{2}(\mathrm{ppm})\end{array}$ & $\begin{array}{c}\text { deviation } \\
\Delta \delta=\delta_{1}-\delta_{2} \\
(\mathrm{ppm})\end{array}$ \\
\hline 2 & 137.7 & 137.4 & 0.3 \\
3 & 80.0 & 79.7 & 0.3 \\
5 & 40.9 & 40.6 & 0.3 \\
6 & 24.5 & 24.2 & 0.3 \\
7 & 110.1 & 109.8 & 0.3 \\
8 & 129.5 & 129.2 & 0.3 \\
9 & 118.8 & 118.5 & 0.3 \\
10 & 120.9 & 120.6 & 0.3 \\
11 & 122.2 & 121.9 & 0.3 \\
12 & 109.9 & 109.5 & 0.4 \\
13 & 132.0 & 131.7 & 0.3 \\
14 & 34.9 & 34.6 & 0.3 \\
15 & 23.5 & 23.2 & 0.3 \\
16 & 49.7 & 49.4 & 0.3 \\
17 & 24.4 & 24.1 & 0.3 \\
18 & 12.9 & 12.6 & 0.3 \\
19 & 24.7 & 24.4 & 0.3 \\
20 & 36.4 & 36.1 & 0.3 \\
21 & 58.8 & 58.5 & 0.3 \\
$\mathrm{CO}_{2} \mathrm{Me}$ & 176.7 & 176.4 & 0.3 \\
$\mathrm{CO}_{2} \mathrm{Me}$ & 53.2 & 52.9 & 0.3
\end{tabular}


4.5 Comparison of Spectroscopic Data of Natural (+)-dippinine C (5) and our Synthetic (-)dippinine $\mathrm{C}(5)$

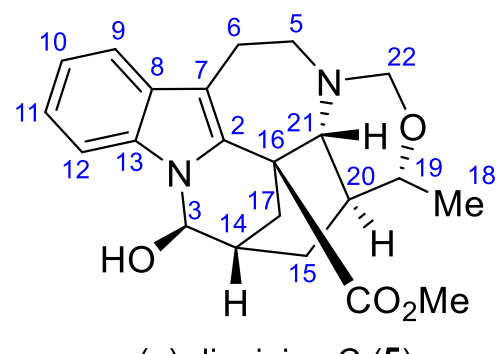

Table S9. Comparison of ${ }^{1} \mathrm{H}$ NMR spectroscopic data of natural (+)-dippinine C (5) and our synthetic (-)-dippinine C (5)

\begin{tabular}{cccc}
\hline position & $\begin{array}{c}\text { natural } \mathbf{5}^{11} \\
\delta_{1}(\mathrm{ppm} ; \text { multi, } J \text { in Hz) }\end{array}$ & $\begin{array}{c}\text { synthetic } 5 \\
\delta_{2}(\mathrm{ppm} ; \text { multi, } J \text { in Hz) }\end{array}$ & $\begin{array}{c}\text { deviation } \\
\Delta \delta=\delta_{1}-\delta_{2} \\
(\mathrm{ppm})\end{array}$ \\
\hline 3 & $5.58(\mathrm{~m})$ & $5.57(\mathrm{~s})$ & 0.01 \\
$5 \mathrm{a}$ & $2.79(\mathrm{~m})$ & $2.77-2.82(\mathrm{~m})$ & 0 \\
$5 \mathrm{~b}$ & $2.79(\mathrm{~m})$ & $2.77-2.82(\mathrm{~m})$ & 0 \\
$6 \mathrm{a}$ & $2.74(\mathrm{~m})$ & $2.72-2.76(\mathrm{~m})$ & 0 \\
$6 \mathrm{~b}$ & $2.93(\mathrm{~m})$ & $2.94(\mathrm{~d}, 5.0)$ & -0.01 \\
9 & $7.56(\mathrm{ddd}, 7.0,1.5,0.6)$ & $7.54(\mathrm{~d}, 6.1)$ & 0.02 \\
10 & $7.20(\mathrm{td}, 7.0,1.5)$ & $7.21(\mathrm{t}, 1.9)$ & -0.01 \\
11 & $7.23(\mathrm{td}, 7.0,1.5)$ & $7.23(\mathrm{~d}, 1.8)$ & 0 \\
12 & $7.53(\mathrm{ddd}, 7.0,1.5,0.6)$ & $7.49(\mathrm{~d}, 7.0)$ & 0.04 \\
14 & $2.46(\mathrm{~m})$ & $2.43-2.47(\mathrm{~m})$ & 0.01 \\
$15 \mathrm{a}$ & $1.23(\mathrm{ddd}, 14.0,11.0,6.0)$ & $1.24-1.26(\mathrm{~m})$ & -0.02 \\
$15 \mathrm{~b}$ & $1.67(\mathrm{ddt}, 14.0,5.5,2.5)$ & $1.66(\mathrm{dd}, 14.4,5.4)$ & 0.01 \\
$17 \mathrm{a}$ & $2.68(\mathrm{dt}, 13.0,2.5)$ & $2.66(\mathrm{dt}, 13.3,2.4)$ & 0.02 \\
$17 \mathrm{~b}$ & $2.02(\mathrm{dd}, 13.0,4.0)$ & $2.03(\mathrm{~d}, 3.7)$ & -0.01 \\
18 & $0.93(\mathrm{~d}, 6.0)$ & $0.91(\mathrm{~d}, 6.2)$ & 0.02 \\
19 & $3.27(\mathrm{dq}, 9.0,6.0)$ & $3.25(\mathrm{dd}, 9.0,6.1)$ & 0.02 \\
20 & $0.67(\mathrm{tdd}, 11.0,9.0,5.5)$ & $0.60-0.72(\mathrm{~m})$ & 0.01 \\
21 & $3.44(\mathrm{~d}, 11.0)$ & $3.43(\mathrm{~d}, 11.0)$ & 0.01 \\
$22 \mathrm{a}$ & $4.62(\mathrm{~d}, 10.5)$ & $4.61(\mathrm{~d}, 10.5)$ & 0.01 \\
$22 \mathrm{~b}$ & $4.41(\mathrm{~d}, 10.5)$ & $4.40(\mathrm{~d}, 10.5)$ & 0.01 \\
$\mathrm{CO}_{2} \mathrm{Me}$ & $3.80(\mathrm{~s})$ & $3.79(\mathrm{~s})$ & 0.01
\end{tabular}


Table S10. Comparison of ${ }^{13} \mathrm{C}$ NMR spectroscopic data of natural (+)-dippinine C (5) and our synthetic (-)-dippinine C (5)

\begin{tabular}{cccc}
\hline position & $\begin{array}{c}\text { natural } \mathbf{5}^{11} \\
\delta_{1}(\mathrm{ppm})\end{array}$ & $\begin{array}{c}\text { synthetic } \mathbf{5} \\
\delta_{2}(\mathrm{ppm})\end{array}$ & $\begin{array}{c}\text { deviation } \\
\Delta \delta^{-} \delta_{1}-\delta_{2} \\
(\mathrm{ppm})\end{array}$ \\
\hline 2 & 132.6 & 132.8 & -0.2 \\
3 & 79.4 & 79.7 & -0.3 \\
5 & 47.6 & 47.8 & -0.2 \\
6 & 23.2 & 23.4 & -0.2 \\
7 & 113.9 & 114.2 & -0.3 \\
8 & 128.1 & 128.4 & -0.3 \\
9 & 109.9 & 110.0 & -0.1 \\
10 & 120.8 & 121.1 & -0.3 \\
11 & 121.8 & 122.0 & -0.2 \\
12 & 118.7 & 119.0 & -0.3 \\
13 & 136.8 & 137.0 & -0.2 \\
14 & 36.0 & 36.1 & -0.1 \\
15 & 31.3 & 31.5 & -0.2 \\
16 & 47.7 & 47.9 & -0.2 \\
17 & 29.0 & 29.2 & -0.2 \\
18 & 18.5 & 18.8 & -0.3 \\
19 & 79.4 & 79.6 & -0.2 \\
20 & 33.1 & 33.3 & -0.2 \\
21 & 64.6 & 64.8 & -0.2 \\
22 & 88.7 & 88.9 & -0.2 \\
$\mathrm{CO}_{2} \mathrm{Me}$ & 52.6 & 52.9 & -0.3 \\
$\mathrm{CO}_{2} \mathrm{Me}$ & 173.5 & 173.7 & -0.2 \\
& & &
\end{tabular}


4.6 Comparison of Spectroscopic Data of Natural (+)-tronocarpine (6) and Our Synthetic (-)tronocarpine (6)

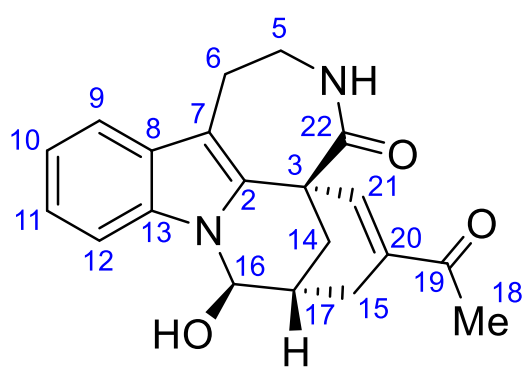

(-)-tronocarpine (6)

Table S11. Comparison of ${ }^{1} \mathrm{H}$ NMR spectroscopic data of natural (+)-tronocarpine (6) and our synthetic (-)-tronocarpine (6)

\begin{tabular}{cccc}
\hline position & natural $\mathbf{6}^{12}$ & synthetic 6 & $\begin{array}{c}\text { deviation } \\
\Delta \delta=\delta_{1}-\end{array}$ \\
\hline $5 \mathrm{a}$ & $\delta_{1}(\mathrm{ppm} ;$ multi, $J$ in $\mathrm{Hz})$ & $\delta_{2}(\mathrm{ppm} ;$ multi, $J$ in $\mathrm{Hz})$ & $\delta_{2}(\mathrm{ppm})$ \\
\hline $5 \mathrm{~b}$ & $3.72(\mathrm{dddd}, 14.7,12.7,7.0,3.5)$ & $3.75(\mathrm{dddd}, 14.7,12.6,6.4,2.2)$ & -0.05 \\
$6 \mathrm{a}$ & $2.63(\mathrm{ddd}, 16.0,12.7,3.5)$ & $2.61-2.70(\mathrm{~m})$ & -0.02 \\
$6 \mathrm{~b}$ & $2.86(\mathrm{dt}, 16.0,3.5)$ & $2.80-2.93(\mathrm{~m})$ & -0.01 \\
9 & $7.45(\mathrm{ddd}, 8.0,1.1,0.8)$ & $7.44(\mathrm{~d}, 7.8)$ & 0.01 \\
10 & $7.17(\mathrm{ddd}, 8.0,7.0,1.1)$ & $7.16(\mathrm{ddd}, 8.0,7.1,1.0)$ & 0.01 \\
11 & $7.26(\mathrm{ddd}, 8.0,7.0,1.1)$ & $7.21-7.29(\mathrm{~m})$ & 0.01 \\
12 & $7.49(\mathrm{ddd}, 8.0,1.1,0.8)$ & $7.47(\mathrm{~d}, 8.2)$ & 0.02 \\
$14 \mathrm{a}$ & $2.15(\mathrm{dd}, 14.0,3.6)$ & $2.13-2.19(\mathrm{~m})$ & -0.01 \\
$14 \mathrm{~b}$ & $2.67(\mathrm{dt}, 14.0,2.0)$ & $2.69-2.77(\mathrm{~m})$ & -0.06 \\
$15 \mathrm{a}$ & $2.28(\mathrm{dd}, 19.0,1.8)$ & $2.29(\mathrm{dd}, 19.0,2.1)$ & -0.01 \\
$15 \mathrm{~b}$ & $2.79(\mathrm{ddd}, 19.0,9.0,1.6)$ & $2.80-2.93(\mathrm{~m})$ & -0.08 \\
16 & $5.67(\mathrm{br})$ & $5.69(\mathrm{~d}, 7.0)$ & -0.02 \\
17 & $2.84(\mathrm{~m})$ & $2.80-2.93(\mathrm{~m})$ & -0.02 \\
18 & $2.18(\mathrm{~s})$ & $2.18(\mathrm{~s})$ & 0 \\
21 & $7.05(\mathrm{~m})$ & $7.02-7.07(\mathrm{~m})$ & 0 \\
$\mathrm{NH}$ & $6.01(\mathrm{t}, 7.0)$ & $5.87(\mathrm{t}, 7.1)$ & 0.14
\end{tabular}


Table S12. Comparison of ${ }^{13} \mathrm{C}$ NMR spectroscopic data of natural (+)-tronocarpine (6) and our synthetic (-)-tronocarpine (6)

\begin{tabular}{cccc}
\hline position & $\begin{array}{c}\text { natural } \mathbf{6}^{12} \\
\delta_{1}(\mathrm{ppm})\end{array}$ & $\begin{array}{c}\text { synthetic } \mathbf{6} \\
\delta_{2}(\mathrm{ppm})\end{array}$ & $\begin{array}{c}\text { deviation } \\
\Delta \delta=\delta_{1}-\delta_{2} \\
(\mathrm{ppm})\end{array}$ \\
\hline 2 & 127.4 & 127.7 & -0.3 \\
3 & 43.9 & 44.2 & -0.3 \\
5 & 40.8 & 41.2 & -0.4 \\
6 & 25.2 & 25.6 & -0.4 \\
7 & 110.9 & 111.4 & -0.5 \\
8 & 128.2 & 128.6 & -0.4 \\
9 & 118.2 & 118.6 & -0.4 \\
10 & 120.6 & 120.9 & -0.3 \\
11 & 122.8 & 123.2 & -0.4 \\
12 & 110.6 & 110.5 & 0.1 \\
13 & 135.9 & 136.0 & -0.1 \\
14 & 25.4 & 25.7 & -0.3 \\
15 & 26.3 & 26.6 & -0.3 \\
16 & 80.5 & 80.9 & -0.4 \\
17 & 32.8 & 32.9 & -0.1 \\
18 & 25.2 & 25.5 & -0.3 \\
19 & 198.2 & 198.3 & -0.1 \\
20 & 137.9 & 138.1 & -0.2 \\
21 & 139.7 & 139.8 & -0.1 \\
22 & 174.5 & 174.4 & 0.1 \\
& & &
\end{tabular}


4.7 Comparison of Spectroscopic Data of Natural (-)-coronaridine (S11) and Our Synthetic (+)coronaridine (S11)

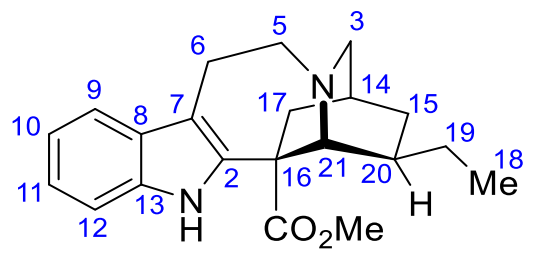

$(+)$-coronaridine (S11)

Table S13. Comparison of ${ }^{1} \mathrm{H}$ NMR spectroscopic data of natural (-)-coronaridine (S11) and our synthetic (+)-coronaridine (S11)

\begin{tabular}{cccc}
\hline position & $\begin{array}{c}\text { natural S11 }{ }^{14} \\
\delta_{1}(\mathrm{ppm} ; \text { multi, } J \text { in Hz) }\end{array}$ & $\begin{array}{c}\text { synthetic S11 } \\
\delta_{2}(\mathrm{ppm} ; \text { multi, } J \text { in Hz) }\end{array}$ & $\begin{array}{c}\text { deviation } \\
\Delta \delta=\delta_{1}-\delta_{2} \\
(\mathrm{ppm})\end{array}$ \\
\hline $3 \mathrm{a}$ & $2.93(\mathrm{~m})$ & $2.90(\mathrm{dt}, 8.5,3.0)$ & 0.03 \\
$3 \mathrm{~b}$ & $2.81(\mathrm{br} \mathrm{d}, 8.0)$ & $2.80(\mathrm{~d}, 8.6)$ & 0.01 \\
$5 \mathrm{a}$ & $3.38(\mathrm{~m})$ & $3.38(\mathrm{ddd}, 15.3,8.6,5.8)$ & 0 \\
$5 \mathrm{~b}$ & $3.15-3.23(\mathrm{~m})$ & $3.19(\mathrm{dddd}, 13.8,12.1,7.6,5.9)$ & 0 \\
$6 \mathrm{a}$ & $3.15-3.23(\mathrm{~m})$ & $3.19(\mathrm{dddd}, 13.8,12.1,7.6,5.9)$ & 0 \\
$6 \mathrm{~b}$ & $3.02(\mathrm{~m})$ & $2.96-3.05(\mathrm{~m})$ & 0.01 \\
9 & $7.24(\mathrm{~d}, 8.0)$ & $7.23(\mathrm{~d}, 7.4)$ & 0.01 \\
10 & $7.14(\mathrm{t})$ & $7.13(\mathrm{ddd}, 8.1,7.0,1.2)$ & 0.01 \\
11 & $7.08(\mathrm{t})$ & $7.07(\mathrm{ddd}, 8.0,6.9,1.1)$ & 0.01 \\
12 & $7.48(\mathrm{~d}, 8.0)$ & $7.47(\mathrm{~d}, 7.8)$ & 0.01 \\
14 & $1.91(\mathrm{br} \mathrm{s})$ & $1.85-1.93(\mathrm{~m})$ & 0.02 \\
$15 \mathrm{a}$ & $1.88(\mathrm{br} \mathrm{s})$ & $1.73(\mathrm{dddd}, 12.2,9.8,4.3,2.1)$ & 0.15 \\
$15 \mathrm{~b}$ & $1.59(\mathrm{~m})$ & $1.57(\mathrm{dt}, 13.3,7.4)$ & 0.02 \\
$17 \mathrm{a}$ & $2.58(\mathrm{br} \mathrm{d}, 13.0)$ & $2.54-2.60(\mathrm{~m})$ & 0.01 \\
$17 \mathrm{~b}$ & $2.09(\mathrm{~m})$ & $1.85-1.93(\mathrm{~m})$ & 0.2 \\
18 & $0.90(\mathrm{t})$ & $0.89(\mathrm{t}, 7.4)$ & 0.01 \\
$19 \mathrm{a}$ & $1.44(\mathrm{~m})$ & $1.43(\mathrm{ddd}, 13.6,7.6,6.4)$ & 0.01 \\
$19 \mathrm{~b}$ & $1.32(\mathrm{~m})$ & $1.32(\mathrm{dq}, 9.4,7.1)$ & 0 \\
20 & $1.13(\mathrm{~m})$ & $1.09-1.15(\mathrm{~m})$ & 0.01 \\
21 & $3.56(\mathrm{br} \mathrm{s})$ & $3.55(\mathrm{br} \mathrm{s})$ & 0.01 \\
$\mathrm{CO}{ }_{2} \mathrm{Me}$ & $3.71(\mathrm{~s})$ & $3.70(\mathrm{~s})$ & 0.01 \\
$\mathrm{NH}$ & $7.75(\mathrm{br} \mathrm{s})$ & $7.80(\mathrm{br} \mathrm{s})$ & -0.05 \\
& & &
\end{tabular}


Table S14. Comparison of ${ }^{13} \mathrm{C}$ NMR spectroscopic data of natural (-)-coronaridine (S10) and our synthetic (+)-coronaridine (S11)

\begin{tabular}{|c|c|c|c|}
\hline position & $\begin{array}{c}\text { natural S11 }^{14} \\
\delta_{1}(\mathrm{ppm})\end{array}$ & $\begin{array}{c}\text { synthetic S11 } \\
\delta_{2}(\mathrm{ppm})\end{array}$ & $\begin{array}{c}\text { deviation } \\
\Delta \delta=\delta_{1}-\delta_{2} \\
(p p m)\end{array}$ \\
\hline 2 & 136.5 & 136.6 & -0.1 \\
\hline 3 & 53.1 & 53.2 & -0.1 \\
\hline 5 & 51.5 & 51.6 & -0.1 \\
\hline 6 & 22.2 & 22.1 & 0.1 \\
\hline 7 & 110.3 & 110.3 & 0 \\
\hline 8 & 128.8 & 128.8 & 0 \\
\hline 9 & 118.3 & 118.5 & -0.2 \\
\hline 10 & 119.0 & 119.2 & -0.2 \\
\hline 11 & 121.8 & 122.0 & -0.2 \\
\hline 12 & 110.3 & 110.4 & -0.1 \\
\hline 13 & 135.6 & 135.5 & 0.1 \\
\hline 14 & 27.2 & 27.4 & -0.2 \\
\hline 15 & 32.0 & 32.1 & -0.1 \\
\hline 16 & 55.1 & 55.1 & 0 \\
\hline 17 & 36.3 & 36.5 & -0.2 \\
\hline 18 & 11.6 & 11.7 & -0.1 \\
\hline 19 & 26.6 & 26.8 & -0.2 \\
\hline 20 & 38.9 & 39.2 & -0.3 \\
\hline 21 & 57.2 & 57.5 & -0.3 \\
\hline$\underline{\mathrm{CO}_{2}} \mathrm{Me}$ & 175.9 & 175.8 & 0.1 \\
\hline $\mathrm{CO}_{2} \underline{\mathrm{Me}}$ & 52.4 & 52.6 & -0.2 \\
\hline
\end{tabular}


4.8 Comparison of Spectroscopic Data of Natural (-)-isovoacangine (44) and Our Synthetic (+)isovoacangine (44)

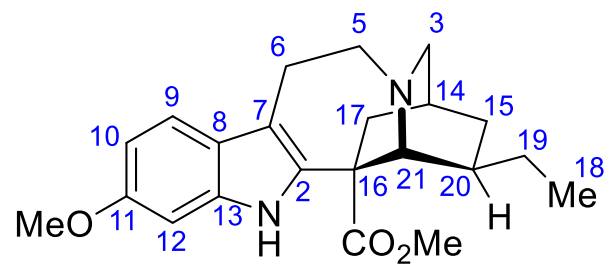

$(+)$-isovoacangine $(44)$

Table S15. Comparison of ${ }^{1} \mathrm{H}$ NMR spectroscopic data of natural (-)-isovoacangine (44) and our synthetic (+)-isovoacangine (44)

\begin{tabular}{cccc}
\hline position & $\begin{array}{c}\text { natural 44 } \\
\delta_{1}(\mathrm{ppm} ; \text { multi, } J \text { in Hz) }\end{array}$ & $\begin{array}{c}\text { synthetic } \mathbf{4 4} \\
\delta_{2}(\mathrm{ppm} ; \text { multi, } \mathrm{J} \text { in Hz) }\end{array}$ & $\begin{array}{c}\text { deviation } \\
\Delta \delta=\delta_{1}-\delta_{2} \\
(\mathrm{ppm})\end{array}$ \\
\hline $3 \mathrm{a}$ & $2.78(\mathrm{dt}, 8.5,1.8)$ & $2.80(\mathrm{~d}, 8.4)$ & -0.02 \\
$3 \mathrm{~b}$ & $2.92(\mathrm{~m})$ & $2.88(\mathrm{~d}, 7.0)$ & 0.04 \\
$5 \mathrm{a}$ & $3.07(\mathrm{~m})$ & $3.18(\mathrm{~d}, 17.3)$ & -0.11 \\
$5 \mathrm{~b}$ & $3.38(\mathrm{~m})$ & $3.30-3.40(\mathrm{~m})$ & 0.03 \\
$6 \mathrm{a}$ & $2.99(\mathrm{~m})$ & $2.98-2.99(\mathrm{~m})$ & 0 \\
$6 \mathrm{~b}$ & $3.06(\mathrm{~m})$ & $3.12(\mathrm{td}, 8.2,6.0)$ & -0.06 \\
9 & $7.13(\mathrm{~d}, 8.7)$ & $7.31(\mathrm{~d}, 9.3)$ & -0.18 \\
10 & $6.69(\mathrm{dd}, 8.7,2.4)$ & $6.71-6.76(\mathrm{~m})$ & -0.04 \\
12 & $6.90(\mathrm{~d}, 2.4)$ & $6.71-6.76(\mathrm{~m})$ & -0.13 \\
14 & $1.84(\mathrm{~m})$ & $1.83-1.91(\mathrm{~m})$ & -0.03 \\
$15 \mathrm{a}$ & $1.13(\mathrm{~m})$ & $1.12(\mathrm{~d}, 11.9)$ & 0.01 \\
$15 \mathrm{~b}$ & $1.76(\mathrm{~m})$ & $1.64-1.77(\mathrm{~m})$ & 0.05 \\
$17 \mathrm{a}$ & $1.92(\mathrm{~m})$ & $1.83-1.91(\mathrm{~m})$ & 0.05 \\
$17 \mathrm{~b}$ & $2.70(\mathrm{dt}, 13.5,2.2)$ & $2.48-2.58(\mathrm{~m})$ & 0.17 \\
18 & $0.90(\mathrm{~d}, 7.4)$ & $0.88(\mathrm{t}, 7.4)$ & 0.02 \\
$19 \mathrm{a}$ & $1.44(\mathrm{~m})$ & $1.37-1.47(\mathrm{~m})$ & 0.02 \\
$19 \mathrm{~b}$ & $1.56(\mathrm{~m})$ & $1.51-1.59(\mathrm{~m})$ & 0.01 \\
20 & $1.37(\mathrm{~m})$ & $1.24-1.34(\mathrm{~m})$ & 0.08 \\
21 & $3.59(\mathrm{br} \mathrm{s})$ & $3.51(\mathrm{~s})$ & 0.08 \\
$\mathrm{CO}{ }_{2} \mathrm{Me}$ & $3.69(\mathrm{~s})$ & $3.70(\mathrm{~s})$ & -0.01 \\
$\mathrm{ArOMe}$ & $3.80(\mathrm{~s})$ & $3.81(\mathrm{~s})$ & -0.01 \\
$\mathrm{NH}$ & - & $7.67(\mathrm{~s})$ & - \\
& & &
\end{tabular}


Table S16. Comparison of ${ }^{13} \mathrm{C}$ NMR spectroscopic data of natural (-)-isovoacangine (44) and our synthetic (+)-isovoacangine (44)

\begin{tabular}{cccc}
\hline position & $\begin{array}{c}\text { natural } \mathbf{4 4} \mathbf{1 5}^{15} \\
\delta_{1}(\mathrm{ppm})\end{array}$ & $\begin{array}{c}\text { synthetic } \mathbf{4 4} \\
\delta_{2}(\mathrm{ppm})\end{array}$ & $\begin{array}{c}\text { deviation } \\
\Delta \delta^{-} \delta_{1}-\delta_{2} \\
(\mathrm{ppm})\end{array}$ \\
\hline 2 & 136.2 & 136.4 & -0.2 \\
3 & 51.4 & 51.6 & -0.2 \\
5 & 53.1 & 53.3 & -0.2 \\
6 & 22.1 & 22.4 & -0.3 \\
7 & 110.0 & 110.3 & -0.3 \\
8 & 123.2 & 123.4 & -0.2 \\
9 & 119.0 & 119.3 & -0.3 \\
10 & 108.9 & 109.2 & -0.3 \\
11 & 156.4 & 156.7 & -0.3 \\
12 & 94.2 & 94.4 & -0.2 \\
13 & 135.3 & 135.4 & -0.1 \\
14 & 27.3 & 27.6 & -0.3 \\
15 & 32.0 & 32.3 & -0.3 \\
16 & 55.0 & 55.2 & -0.2 \\
17 & 36.4 & 36.6 & -0.2 \\
18 & 11.6 & 11.9 & -0.3 \\
19 & 26.7 & 26.9 & -0.2 \\
20 & 39.1 & 39.4 & -0.3 \\
21 & 57.6 & 57.9 & -0.3 \\
$\mathrm{CO}_{2} \mathrm{Me}$ & 52.4 & 52.8 & -0.4 \\
$\mathrm{CO}_{2} \mathrm{Me}$ & 175.8 & 176.1 & -0.3 \\
$\mathrm{ArOMe}$ & 55.7 & 55.9 & -0.2 \\
& & &
\end{tabular}


5. X-ray Crystal Structure Determination of Purported (+)-Tabercarpamine G (27)

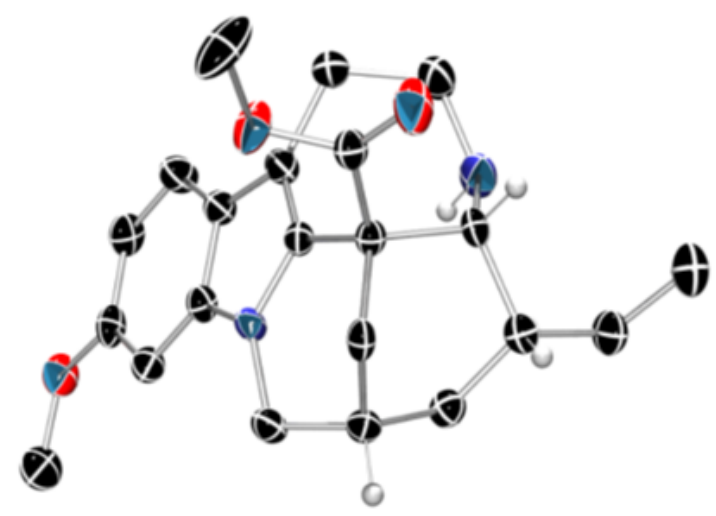

front view of 27

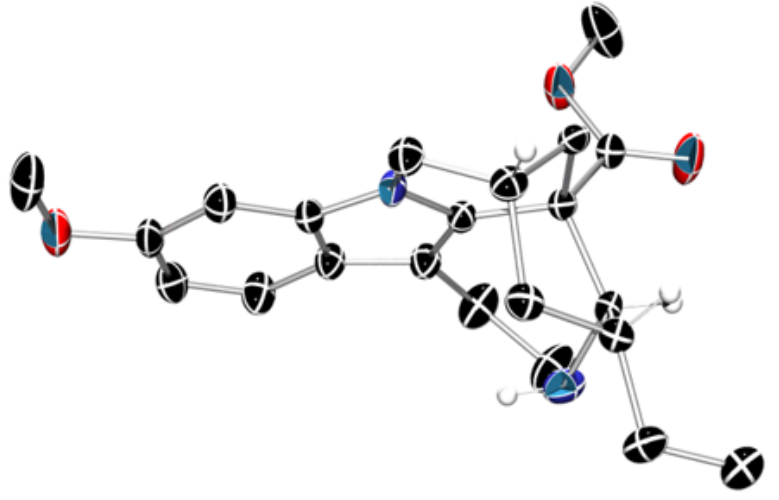

bottom view of 27

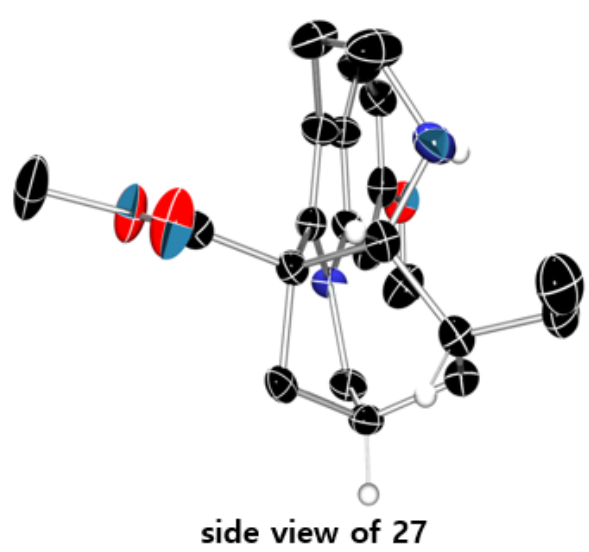

side view of 27

Figure S2. Thermal ellipsoid representation of 27. 
Table S17. Crystal data and structure refinement for 27.

Empirical formula

Formula weight

Temperature

Wavelength

Crystal system

Space group

Unit cell dimensions

Volume

Z

Density (calculated)

Absorption coefficient

$\mathrm{F}(000)$

Crystal size

Theta range for data collection

Index ranges

Reflections collected

Independent reflections

Completeness to theta $=25.242^{\circ}$

Absorption correction

Max. and min. transmission

Refinement method

Data / restraints / parameters

Goodness-of-fit on $\mathrm{F}^{2}$

Final $\mathrm{R}$ indices [l>2sigma(I)]

$\mathrm{R}$ indices (all data)

Absolute structure parameter

Largest diff. peak and hole
$\mathrm{C}_{22} \mathrm{H}_{28} \mathrm{~N}_{2} \mathrm{O}_{3}$

368.46

173(2) K

$0.71073 \AA$

Monoclinic

$P 2_{1}$

$\begin{array}{ll}a=7.3041(4) \AA & \alpha=90^{\circ} \\ b=8.6210(5) \AA & \beta=102.2247(18)^{\circ} \\ c=15.8277(10) \AA & \gamma=90^{\circ}\end{array}$

974.05(10) $\AA^{3}$

2

$1.256 \mathrm{Mg} / \mathrm{m}^{3}$

$0.084 \mathrm{~mm}^{-1}$

396

$0.132 \times 0.039 \times 0.037 \mathrm{~mm}^{3}$

3.450 to $25.497^{\circ}$.

$-8<=\mathrm{h}<=8,-10<=\mathrm{k}<=10,-19<=\mathrm{k}<=19$

12151

$3587[\mathrm{R}(\mathrm{int})=0.0647]$

$99.3 \%$

Semi-empirical from equivalents

0.7455 and 0.6161

Full-matrix least-squares on $\mathrm{F}^{2}$

3587 / 1 / 250

1.127

$\mathrm{R} 1=0.0567, \mathrm{wR} 2=0.1300$

$\mathrm{R} 1=0.0652, \mathrm{wR} 2=0.1348$

$0.5(9)$

0.211 and $-0.205 e \cdot \AA^{-3}$ 
Table S18. Atomic coordinates ( $\left.x 1^{4}\right)$ and equivalent isotropic displacement parameters $\left(\AA^{2} x\right.$ $10^{3}$ ) for $27 . \mathrm{U}(\mathrm{eq})$ is defined as one third of the trace of the orthogonalized $\mathrm{U}^{\mathrm{ij}}$ tensor.

\begin{tabular}{|c|c|c|c|c|}
\hline & $x$ & $y$ & z & $\mathrm{U}(\mathrm{eq})$ \\
\hline$C(1)$ & $5506(8)$ & $6247(7)$ & $6483(3)$ & $46(1)$ \\
\hline $\mathrm{O}(2)$ & $6947(5)$ & $5144(4)$ & $6434(2)$ & $36(1)$ \\
\hline$C(3)$ & $6206(6)$ & $5486(5)$ & $4868(3)$ & $27(1)$ \\
\hline$C(4)$ & $7204(6)$ & $4784(5)$ & $5620(3)$ & $28(1)$ \\
\hline$C(5)$ & $8583(6)$ & $3672(6)$ & $5588(3)$ & $33(1)$ \\
\hline$C(6)$ & $9014(6)$ & $3208(6)$ & $4818(3)$ & $33(1)$ \\
\hline$C(7)$ & $8034(6)$ & $3897(5)$ & $4038(3)$ & $25(1)$ \\
\hline$C(8)$ & $6649(6)$ & $5004(5)$ & $4097(3)$ & $24(1)$ \\
\hline$N(9)$ & $5881(5)$ & $5514(4)$ & $3269(2)$ & $22(1)$ \\
\hline$C(10)$ & $4729(6)$ & $6911(5)$ & $3063(3)$ & $26(1)$ \\
\hline$C(11)$ & $4996(6)$ & $7541(5)$ & $2207(3)$ & $24(1)$ \\
\hline$C(12)$ & $4637(6)$ & $6206(5)$ & $1547(3)$ & $23(1)$ \\
\hline$C(13)$ & $6341(5)$ & $5124(5)$ & $1744(2)$ & $21(1)$ \\
\hline$C(14)$ & $6765(5)$ & $4735(5)$ & $2701(2)$ & $21(1)$ \\
\hline$C(15)$ & $8088(6)$ & $3752(5)$ & $3138(3)$ & $26(1)$ \\
\hline$C(16)$ & $9425(7)$ & $2862(6)$ & $2723(3)$ & $37(1)$ \\
\hline$C(17)$ & $10321(7)$ & $3850(6)$ & $2114(4)$ & $44(1)$ \\
\hline $\mathrm{N}(18)$ & $9916(5)$ & $5500(5)$ & 2046(3) & $35(1)$ \\
\hline$C(19)$ & $8097(6)$ & $5941(5)$ & 1503(3) & $24(1)$ \\
\hline$C(20)$ & $7847(6)$ & $7726(5)$ & 1504(3) & $27(1)$ \\
\hline$C(21)$ & $6990(6)$ & $8220(5)$ & $2265(3)$ & $28(1)$ \\
\hline $\mathrm{C}(22)$ & $5789(6)$ & $3704(5)$ & $1178(3)$ & $25(1)$ \\
\hline $\mathrm{O}(23)$ & $5991(5)$ & $3570(4)$ & $452(2)$ & $45(1)$ \\
\hline $\mathrm{O}(24)$ & $4850(5)$ & $2651(4)$ & 1542(2) & $37(1)$ \\
\hline$C(25)$ & $4101(9)$ & $1360(6)$ & 1003(3) & $52(2)$ \\
\hline$C(26)$ & $9595(6)$ & $8629(6)$ & $1435(3)$ & $35(1)$ \\
\hline $\mathrm{C}(27)$ & $10228(7)$ & $8308(7)$ & $598(3)$ & $42(1)$ \\
\hline
\end{tabular}


Table S19. Bond lengths $[\AA ̊]$ and angles $\left[{ }^{\circ}\right]$ for 27.

\begin{tabular}{|c|c|}
\hline$\overline{\mathrm{C}(1)-\mathrm{O}(2)}$ & $1.433(6)$ \\
\hline$C(1)-H(1 A)$ & 0.9800 \\
\hline $\mathrm{C}(1)-\mathrm{H}(1 \mathrm{~B})$ & 0.9800 \\
\hline$C(1)-H(1 C)$ & 0.9800 \\
\hline $\mathrm{O}(2)-\mathrm{C}(4)$ & $1.376(5)$ \\
\hline$C(3)-C(8)$ & $1.391(6)$ \\
\hline$C(3)-C(4)$ & $1.395(6)$ \\
\hline $\mathrm{C}(3)-\mathrm{H}(3)$ & 0.9500 \\
\hline$C(4)-C(5)$ & $1.399(7)$ \\
\hline$C(5)-C(6)$ & $1.380(6)$ \\
\hline $\mathrm{C}(5)-\mathrm{H}(5)$ & 0.9500 \\
\hline$C(6)-C(7)$ & $1.420(6)$ \\
\hline $\mathrm{C}(6)-\mathrm{H}(6)$ & 0.9500 \\
\hline$C(7)-C(8)$ & $1.409(6)$ \\
\hline$C(7)-C(15)$ & $1.437(6)$ \\
\hline $\mathrm{C}(8)-\mathrm{N}(9)$ & $1.383(5)$ \\
\hline $\mathrm{N}(9)-\mathrm{C}(14)$ & $1.386(5)$ \\
\hline $\mathrm{N}(9)-\mathrm{C}(10)$ & $1.466(5)$ \\
\hline$C(10)-C(11)$ & $1.509(6)$ \\
\hline $\mathrm{C}(10)-\mathrm{H}(10 \mathrm{~A})$ & 0.9900 \\
\hline $\mathrm{C}(10)-\mathrm{H}(10 \mathrm{~B})$ & 0.9900 \\
\hline$C(11)-C(12)$ & $1.539(6)$ \\
\hline$C(11)-C(21)$ & $1.555(6)$ \\
\hline $\mathrm{C}(11)-\mathrm{H}(11)$ & 1.0000 \\
\hline$C(12)-C(13)$ & $1.534(6)$ \\
\hline $\mathrm{C}(12)-\mathrm{H}(12 \mathrm{~A})$ & 0.9900 \\
\hline $\mathrm{C}(12)-\mathrm{H}(12 \mathrm{~B})$ & 0.9900 \\
\hline$C(13)-C(14)$ & $1.518(5)$ \\
\hline$C(13)-C(22)$ & $1.521(6)$ \\
\hline$C(13)-C(19)$ & $1.580(5)$ \\
\hline$C(14)-C(15)$ & $1.359(6)$ \\
\hline$C(15)-C(16)$ & $1.499(6)$ \\
\hline$C(16)-C(17)$ & $1.532(7)$ \\
\hline $\mathrm{C}(16)-\mathrm{H}(16 \mathrm{~A})$ & 0.9900 \\
\hline $\mathrm{C}(16)-\mathrm{H}(16 \mathrm{~B})$ & 0.9900 \\
\hline $\mathrm{C}(17)-\mathrm{N}(18)$ & $1.452(7)$ \\
\hline $\mathrm{C}(17)-\mathrm{H}(17 \mathrm{~A})$ & 0.9900 \\
\hline
\end{tabular}




\begin{tabular}{|c|c|}
\hline $\mathrm{C}(17)-\mathrm{H}(17 \mathrm{~B})$ & 0.9900 \\
\hline$N(18)-C(19)$ & $1.471(5)$ \\
\hline $\mathrm{N}(18)-\mathrm{H}(18)$ & $0.90(6)$ \\
\hline$C(19)-C(20)$ & $1.550(6)$ \\
\hline $\mathrm{C}(19)-\mathrm{H}(19)$ & 1.0000 \\
\hline$C(20)-C(26)$ & $1.518(6)$ \\
\hline$C(20)-C(21)$ & $1.530(6)$ \\
\hline $\mathrm{C}(20)-\mathrm{H}(20)$ & 1.0000 \\
\hline $\mathrm{C}(21)-\mathrm{H}(21 \mathrm{~A})$ & 0.9900 \\
\hline $\mathrm{C}(21)-\mathrm{H}(21 \mathrm{~B})$ & 0.9900 \\
\hline $\mathrm{C}(22)-\mathrm{O}(23)$ & $1.193(5)$ \\
\hline $\mathrm{C}(22)-\mathrm{O}(24)$ & $1.340(5)$ \\
\hline $\mathrm{O}(24)-\mathrm{C}(25)$ & $1.438(6)$ \\
\hline $\mathrm{C}(25)-\mathrm{H}(25 \mathrm{~A})$ & 0.9800 \\
\hline $\mathrm{C}(25)-\mathrm{H}(25 \mathrm{~B})$ & 0.9800 \\
\hline $\mathrm{C}(25)-\mathrm{H}(25 \mathrm{C})$ & 0.9800 \\
\hline$C(26)-C(27)$ & $1.518(7)$ \\
\hline $\mathrm{C}(26)-\mathrm{H}(26 \mathrm{~A})$ & 0.9900 \\
\hline $\mathrm{C}(26)-\mathrm{H}(26 \mathrm{~B})$ & 0.9900 \\
\hline $\mathrm{C}(27)-\mathrm{H}(27 \mathrm{~A})$ & 0.9800 \\
\hline $\mathrm{C}(27)-\mathrm{H}(27 \mathrm{~B})$ & 0.9800 \\
\hline $\mathrm{C}(27)-\mathrm{H}(27 \mathrm{C})$ & 0.9800 \\
\hline $\mathrm{O}(2)-\mathrm{C}(1)-\mathrm{H}(1 \mathrm{~A})$ & 109.5 \\
\hline $\mathrm{O}(2)-\mathrm{C}(1)-\mathrm{H}(1 \mathrm{~B})$ & 109.5 \\
\hline$H(1 A)-C(1)-H(1 B)$ & 109.5 \\
\hline $\mathrm{O}(2)-\mathrm{C}(1)-\mathrm{H}(1 \mathrm{C})$ & 109.5 \\
\hline$H(1 A)-C(1)-H(1 C)$ & 109.5 \\
\hline$H(1 B)-C(1)-H(1 C)$ & 109.5 \\
\hline$C(4)-O(2)-C(1)$ & 116.6(4) \\
\hline$C(8)-C(3)-C(4)$ & $116.3(4)$ \\
\hline $\mathrm{C}(8)-\mathrm{C}(3)-\mathrm{H}(3)$ & 121.9 \\
\hline $\mathrm{C}(4)-\mathrm{C}(3)-\mathrm{H}(3)$ & 121.9 \\
\hline $\mathrm{O}(2)-\mathrm{C}(4)-\mathrm{C}(3)$ & $123.4(4)$ \\
\hline $\mathrm{O}(2)-\mathrm{C}(4)-\mathrm{C}(5)$ & $115.5(4)$ \\
\hline$C(3)-C(4)-C(5)$ & 121.1(4) \\
\hline$C(6)-C(5)-C(4)$ & 122.1(4) \\
\hline $\mathrm{C}(6)-\mathrm{C}(5)-\mathrm{H}(5)$ & 118.9 \\
\hline$C(4)-C(5)-H(5)$ & 118.9 \\
\hline$C(5)-C(6)-C(7)$ & 118.6(4) \\
\hline
\end{tabular}




$\begin{array}{ll}\mathrm{C}(5)-\mathrm{C}(6)-\mathrm{H}(6) & 120.7 \\ \mathrm{C}(7)-\mathrm{C}(6)-\mathrm{H}(6) & 120.7 \\ \mathrm{C}(8)-\mathrm{C}(7)-\mathrm{C}(6) & 117.5(4) \\ \mathrm{C}(8)-\mathrm{C}(7)-\mathrm{C}(15) & 107.2(3) \\ \mathrm{C}(6)-\mathrm{C}(7)-\mathrm{C}(15) & 135.3(4) \\ \mathrm{N}(9)-\mathrm{C}(8)-\mathrm{C}(3) & 127.8(4) \\ \mathrm{N}(9)-\mathrm{C}(8)-\mathrm{C}(7) & 107.8(3) \\ \mathrm{C}(3)-\mathrm{C}(8)-\mathrm{C}(7) & 124.4(4) \\ \mathrm{C}(8)-\mathrm{N}(9)-\mathrm{C}(14) & 108.0(3) \\ \mathrm{C}(8)-\mathrm{N}(9)-\mathrm{C}(10) & 124.8(3) \\ \mathrm{C}(14)-\mathrm{N}(9)-\mathrm{C}(10) & 125.3(3) \\ \mathrm{N}(9)-\mathrm{C}(10)-\mathrm{C}(11) & 108.6(3) \\ \mathrm{N}(9)-\mathrm{C}(10)-\mathrm{H}(10 \mathrm{~A}) & 110.0 \\ \mathrm{C}(11)-\mathrm{C}(10)-\mathrm{H}(10 \mathrm{~A}) & 110.0 \\ \mathrm{~N}(9)-\mathrm{C}(10)-\mathrm{H}(10 \mathrm{~B}) & 110.0 \\ \mathrm{C}(11)-\mathrm{C}(10)-\mathrm{H}(10 \mathrm{~B}) & 110.0 \\ \mathrm{H}(10 \mathrm{~A})-\mathrm{C}(10)-\mathrm{H}(10 \mathrm{~B}) & 108.4 \\ \mathrm{C}(10)-\mathrm{C}(11)-\mathrm{C}(12) & 107.7(4) \\ \mathrm{C}(10)-\mathrm{C}(11)-\mathrm{C}(21) & 112.4(3) \\ \mathrm{C}(12)-\mathrm{C}(11)-\mathrm{C}(21) & 110.1(3) \\ \mathrm{C}(10)-\mathrm{C}(11)-\mathrm{H}(11) & 108.9 \\ \mathrm{C}(12)-\mathrm{C}(11)-\mathrm{H}(11) & 108.9 \\ \mathrm{C}(21)-\mathrm{C}(11)-\mathrm{H}(11) & 108.9 \\ \mathrm{C}(13)-\mathrm{C}(12)-\mathrm{C}(11) & 107.6(3) \\ \mathrm{C}(13)-\mathrm{C}(12)-\mathrm{H}(12 \mathrm{~A}) & 110.2 \\ \mathrm{C}(11)-\mathrm{C}(12)-\mathrm{H}(12 \mathrm{~A}) & 110.2 \\ \mathrm{C}(13)-\mathrm{C}(12)-\mathrm{H}(12 \mathrm{~B}) & 110.2 \\ \mathrm{C}(11)-\mathrm{C}(12)-\mathrm{H}(12 \mathrm{~B}) & 110.2 \\ \mathrm{H}(12 \mathrm{~A})-\mathrm{C}(12)-\mathrm{H}(12 \mathrm{~B}) & 108.5 \\ \mathrm{C}(14)-\mathrm{C}(13)-\mathrm{C}(22) & 112.5(3) \\ \mathrm{C}(14)-\mathrm{C}(13)-\mathrm{C}(12) & 108.8(3) \\ \mathrm{C}(22)-\mathrm{C}(13)-\mathrm{C}(12) & 105.5(3) \\ \mathrm{C}(14)-\mathrm{C}(13)-\mathrm{C}(19) & 109.6(3) \\ \mathrm{C}(22)-\mathrm{C}(13)-\mathrm{C}(19) & 110.1(3) \\ \mathrm{C}(12)-\mathrm{C}(13)-\mathrm{C}(19) & 110.2(3) \\ \mathrm{C}(15)-\mathrm{C}(14)-\mathrm{N}(9) & 110.5(3) \\ \mathrm{C}(15)-\mathrm{C}(14)-\mathrm{C}(13) & 128.2(4) \\ \mathrm{C}(14)-\mathrm{C}(15)-\mathrm{C}(13) & 121.0(3) \\ & 106.5(4) \\ & 123.7(4) \\ & \end{array}$




$\begin{array}{ll}\mathrm{C}(7)-\mathrm{C}(15)-\mathrm{C}(16) & 129.5(4) \\ \mathrm{C}(15)-\mathrm{C}(16)-\mathrm{C}(17) & 113.3(4) \\ \mathrm{C}(15)-\mathrm{C}(16)-\mathrm{H}(16 \mathrm{~A}) & 108.9 \\ \mathrm{C}(17)-\mathrm{C}(16)-\mathrm{H}(16 \mathrm{~A}) & 108.9 \\ \mathrm{C}(15)-\mathrm{C}(16)-\mathrm{H}(16 \mathrm{~B}) & 108.9 \\ \mathrm{C}(17)-\mathrm{C}(16)-\mathrm{H}(16 \mathrm{~B}) & 108.9 \\ \mathrm{H}(16 \mathrm{~A})-\mathrm{C}(16)-\mathrm{H}(16 \mathrm{~B}) & 107.7 \\ \mathrm{~N}(18)-\mathrm{C}(17)-\mathrm{C}(16) & 118.9(4) \\ \mathrm{N}(18)-\mathrm{C}(17)-\mathrm{H}(17 \mathrm{~A}) & 107.6 \\ \mathrm{C}(16)-\mathrm{C}(17)-\mathrm{H}(17 \mathrm{~A}) & 107.6 \\ \mathrm{~N}(18)-\mathrm{C}(17)-\mathrm{H}(17 \mathrm{~B}) & 107.6 \\ \mathrm{C}(16)-\mathrm{C}(17)-\mathrm{H}(17 \mathrm{~B}) & 107.6 \\ \mathrm{H}(17 \mathrm{~A})-\mathrm{C}(17)-\mathrm{H}(17 \mathrm{~B}) & 107.0 \\ \mathrm{C}(17)-\mathrm{N}(18)-\mathrm{C}(19) & 116.2(4) \\ \mathrm{C}(17)-\mathrm{N}(18)-\mathrm{H}(18) & 105(4) \\ \mathrm{C}(19)-\mathrm{N}(18)-\mathrm{H}(18) & 109(4) \\ \mathrm{N}(18)-\mathrm{C}(19)-\mathrm{C}(20) & 110.4(3) \\ \mathrm{N}(18)-\mathrm{C}(19)-\mathrm{C}(13) & 115.0(3) \\ \mathrm{C}(20)-\mathrm{C}(19)-\mathrm{C}(13) & 109.9(3) \\ \mathrm{N}(18)-\mathrm{C}(19)-\mathrm{H}(19) & 107.1 \\ \mathrm{C}(20)-\mathrm{C}(19)-\mathrm{H}(19) & 107.1 \\ \mathrm{C}(13)-\mathrm{C}(19)-\mathrm{H}(19) & 107.1 \\ \mathrm{C}(26)-\mathrm{C}(20)-\mathrm{C}(21) & 113.8(4) \\ \mathrm{C}(26)-\mathrm{C}(20)-\mathrm{C}(19) & 114.1(4) \\ \mathrm{C}(21)-\mathrm{C}(20)-\mathrm{C}(19) & 110.2(3) \\ \mathrm{C}(26)-\mathrm{C}(20)-\mathrm{H}(20) & 106.0 \\ \mathrm{C}(21)-\mathrm{C}(20)-\mathrm{H}(20) & 106.0 \\ \mathrm{C}(19)-\mathrm{C}(20)-\mathrm{H}(20) & 106.0 \\ \mathrm{C}(20)-\mathrm{C}(21)-\mathrm{C}(11) & 112.6(3) \\ \mathrm{C}(20)-\mathrm{C}(21)-\mathrm{H}(21 \mathrm{~A}) & 109.1 \\ \mathrm{C}(11)-\mathrm{C}(21)-\mathrm{H}(21 \mathrm{~A}) & 109.1 \\ \mathrm{C}(20)-\mathrm{C}(21)-\mathrm{H}(21 \mathrm{~B}) & 109.1 \\ \mathrm{C}(11)-\mathrm{C}(21)-\mathrm{H}(21 \mathrm{~B}) & 109.1 \\ \mathrm{H}(21 \mathrm{~A})-\mathrm{C}(21)-\mathrm{H}(21 \mathrm{~B}) & 107.8 \\ \mathrm{O}(23)-\mathrm{C}(22)-\mathrm{O}(24)-\mathrm{C}(24) & 121.8(4) \\ \mathrm{O}(23)-\mathrm{C}(22)-\mathrm{C}(13) & 125.0(4) \\ \mathrm{O}(25 \mathrm{~A}) & 112.8(3) \\ & 115.7(4) \\ & 109.5 \\ & 109.5 \\ & \end{array}$




$\begin{array}{ll}\mathrm{H}(25 \mathrm{~A})-\mathrm{C}(25)-\mathrm{H}(25 \mathrm{~B}) & 109.5 \\ \mathrm{O}(24)-\mathrm{C}(25)-\mathrm{H}(25 \mathrm{C}) & 109.5 \\ \mathrm{H}(25 \mathrm{~A})-\mathrm{C}(25)-\mathrm{H}(25 \mathrm{C}) & 109.5 \\ \mathrm{H}(25 \mathrm{~B})-\mathrm{C}(25)-\mathrm{H}(25 \mathrm{C}) & 109.5 \\ \mathrm{C}(27)-\mathrm{C}(26)-\mathrm{C}(20) & 112.7(4) \\ \mathrm{C}(27)-\mathrm{C}(26)-\mathrm{H}(26 \mathrm{~A}) & 109.1 \\ \mathrm{C}(20)-\mathrm{C}(26)-\mathrm{H}(26 \mathrm{~A}) & 109.1 \\ \mathrm{C}(27)-\mathrm{C}(26)-\mathrm{H}(26 \mathrm{~B}) & 109.1 \\ \mathrm{C}(20)-\mathrm{C}(26)-\mathrm{H}(26 \mathrm{~B}) & 109.1 \\ \mathrm{H}(26 \mathrm{~A})-\mathrm{C}(26)-\mathrm{H}(26 \mathrm{~B}) & 107.8 \\ \mathrm{C}(26)-\mathrm{C}(27)-\mathrm{H}(27 \mathrm{~A}) & 109.5 \\ \mathrm{C}(26)-\mathrm{C}(27)-\mathrm{H}(27 \mathrm{~B}) & 109.5 \\ \mathrm{H}(27 \mathrm{~A})-\mathrm{C}(27)-\mathrm{H}(27 \mathrm{~B}) & 109.5 \\ \mathrm{C}(26)-\mathrm{C}(27)-\mathrm{H}(27 \mathrm{C}) & 109.5 \\ \mathrm{H}(27 \mathrm{~A})-\mathrm{C}(27)-\mathrm{H}(27 \mathrm{C}) & 109.5 \\ \mathrm{H}(27 \mathrm{~B})-\mathrm{C}(27)-\mathrm{H}(27 \mathrm{C}) & 109.5\end{array}$

Symmetry transformations used to generate equivalent atoms: 
Table S20. Anisotropic displacement parameters $\left(\AA^{2} \times 10^{3}\right)$ for 27. The anisotropic displacement factor exponent takes the form: $-2 \pi^{2}\left[h^{2} a^{\star 2} U^{11}+\ldots+2 h k a^{*} b^{\star} U^{12}\right]$

\begin{tabular}{|c|c|c|c|c|c|c|}
\hline & $U^{11}$ & $U^{22}$ & $U^{33}$ & $U^{23}$ & $\mathrm{U}^{13}$ & $\mathrm{U}^{12}$ \\
\hline$C(1)$ & $67(4)$ & $44(3)$ & $31(3)$ & $-7(2)$ & $23(2)$ & $0(3)$ \\
\hline $\mathrm{O}(2)$ & $49(2)$ & $39(2)$ & $21(2)$ & $-3(1)$ & $10(1)$ & $-5(2)$ \\
\hline$C(3)$ & $32(2)$ & $26(2)$ & $24(2)$ & $-2(2)$ & $8(2)$ & $-2(2)$ \\
\hline$C(4)$ & $36(2)$ & $33(3)$ & $17(2)$ & $1(2)$ & $7(2)$ & $-9(2)$ \\
\hline$C(5)$ & $35(2)$ & $39(3)$ & $22(2)$ & $9(2)$ & $2(2)$ & $2(2)$ \\
\hline$C(6)$ & $33(2)$ & $38(3)$ & $28(2)$ & $6(2)$ & $7(2)$ & $11(2)$ \\
\hline$C(7)$ & $26(2)$ & $27(2)$ & $22(2)$ & $4(2)$ & $5(2)$ & $4(2)$ \\
\hline$C(8)$ & $23(2)$ & $29(2)$ & $19(2)$ & $2(2)$ & $4(2)$ & $0(2)$ \\
\hline $\mathrm{N}(9)$ & $24(2)$ & $26(2)$ & $18(2)$ & 2(2) & $7(1)$ & $5(2)$ \\
\hline$C(10)$ & $24(2)$ & $30(2)$ & $27(2)$ & $1(2)$ & $8(2)$ & $6(2)$ \\
\hline$C(11)$ & $20(2)$ & $24(2)$ & $30(2)$ & $4(2)$ & $6(2)$ & $4(2)$ \\
\hline$C(12)$ & $18(2)$ & $28(2)$ & $23(2)$ & $6(2)$ & $4(2)$ & $-4(2)$ \\
\hline$C(13)$ & $20(2)$ & $23(2)$ & $19(2)$ & $3(2)$ & $3(2)$ & $-1(2)$ \\
\hline$C(14)$ & $22(2)$ & $21(2)$ & $20(2)$ & $1(2)$ & $6(2)$ & $-1(2)$ \\
\hline$C(15)$ & $25(2)$ & $29(2)$ & $24(2)$ & $2(2)$ & $7(2)$ & $4(2)$ \\
\hline$C(16)$ & $43(3)$ & $29(3)$ & $45(3)$ & $7(2)$ & $21(2)$ & $9(2)$ \\
\hline$C(17)$ & $41(3)$ & $43(3)$ & $56(3)$ & $16(3)$ & $26(2)$ & $14(2)$ \\
\hline$N(18)$ & $23(2)$ & $40(2)$ & $45(2)$ & $7(2)$ & $10(2)$ & $2(2)$ \\
\hline$C(19)$ & $24(2)$ & $29(2)$ & $19(2)$ & $0(2)$ & $9(2)$ & $-3(2)$ \\
\hline$C(20)$ & $23(2)$ & $31(2)$ & $25(2)$ & $0(2)$ & $4(2)$ & $-2(2)$ \\
\hline$C(21)$ & $26(2)$ & $26(2)$ & $30(2)$ & $1(2)$ & $4(2)$ & $-1(2)$ \\
\hline$C(22)$ & $26(2)$ & $27(2)$ & $21(2)$ & $2(2)$ & $5(2)$ & $0(2)$ \\
\hline $\mathrm{O}(23)$ & $70(2)$ & $44(2)$ & $26(2)$ & $-10(2)$ & $23(2)$ & $-19(2)$ \\
\hline $\mathrm{O}(24)$ & $58(2)$ & $30(2)$ & $22(2)$ & $-4(1)$ & $8(1)$ & $-18(2)$ \\
\hline$C(25)$ & $81(4)$ & $28(3)$ & $36(3)$ & $1(2)$ & $-10(3)$ & $-21(3)$ \\
\hline$C(26)$ & $29(2)$ & $34(3)$ & $43(3)$ & $0(2)$ & $12(2)$ & $-8(2)$ \\
\hline$C(27)$ & $40(3)$ & $48(3)$ & $41(3)$ & $4(2)$ & $14(2)$ & $-9(2)$ \\
\hline
\end{tabular}


Table S21. Hydrogen coordinates $\left(\times 10^{4}\right)$ and isotropic displacement parameters $\left(\AA^{2} \times 10^{3}\right)$ for 27.

\begin{tabular}{|c|c|c|c|c|}
\hline & $x$ & $y$ & $z$ & $\mathrm{U}(\mathrm{eq})$ \\
\hline$H(1 A)$ & 5486 & 6448 & 7090 & 68 \\
\hline $\mathrm{H}(1 \mathrm{~B})$ & 4290 & 5832 & 6187 & 68 \\
\hline $\mathrm{H}(1 \mathrm{C})$ & 5756 & 7217 & 6205 & 68 \\
\hline$H(3)$ & 5277 & 6251 & 4883 & 32 \\
\hline $\mathrm{H}(5)$ & 9243 & 3222 & 6112 & 39 \\
\hline $\mathrm{H}(6)$ & 9948 & 2444 & 4811 & 40 \\
\hline$H(10 A)$ & 5108 & 7698 & 3521 & 32 \\
\hline $\mathrm{H}(10 \mathrm{~B})$ & 3393 & 6654 & 3026 & 32 \\
\hline $\mathrm{H}(11)$ & 4051 & 8377 & 2013 & 29 \\
\hline$H(12 A)$ & 3489 & 5633 & 1596 & 27 \\
\hline $\mathrm{H}(12 \mathrm{~B})$ & 4468 & 6620 & 952 & 27 \\
\hline$H(16 A)$ & 8749 & 1985 & 2392 & 45 \\
\hline$H(16 B)$ & 10429 & 2424 & 3180 & 45 \\
\hline$H(17 A)$ & 11697 & 3730 & 2295 & 53 \\
\hline $\mathrm{H}(17 \mathrm{~B})$ & 9946 & 3401 & 1527 & 53 \\
\hline$H(18)$ & $9960(80)$ & $5810(70)$ & $2590(40)$ & 53 \\
\hline $\mathrm{H}(19)$ & 8123 & 5630 & 897 & 28 \\
\hline $\mathrm{H}(20)$ & 6890 & 7978 & 970 & 32 \\
\hline$H(21 A)$ & 6924 & 9366 & 2280 & 33 \\
\hline$H(21 B)$ & 7816 & 7870 & 2811 & 33 \\
\hline$H(25 A)$ & 3381 & 693 & 1314 & 77 \\
\hline$H(25 B)$ & 5131 & 764 & 854 & 77 \\
\hline$H(25 C)$ & 3280 & 1745 & 473 & 77 \\
\hline$H(26 A)$ & 9343 & 9752 & 1473 & 42 \\
\hline $\mathrm{H}(26 \mathrm{~B})$ & 10619 & 8353 & 1929 & 42 \\
\hline $\mathrm{H}(27 \mathrm{~A})$ & 11369 & 8901 & 590 & 64 \\
\hline $\mathrm{H}(27 \mathrm{~B})$ & 9239 & 8618 & 107 & 64 \\
\hline $\mathrm{H}(27 \mathrm{C})$ & 10484 & 7198 & 558 & 64 \\
\hline
\end{tabular}




\section{Supplemental References}

1. Still, W. C.; Kahn, M.; Mitra, A. Rapid chromatographic technique for preparative separations with moderate resolution. J. Org. Chem. 1978, 43, 2923-2925.

2. Pangborn, A. B.; Giardello, M. A.; Grubbs, R. H.; Rosen, R. K.; Timmers, F.J. Safe and Convenient Procedure for Solvent Purification. Organometallics, 1996, 15, 1518-1520.

3. Specific rotation of natural $(-)-2$ from the report is $[\alpha]^{25} \mathrm{D}:-43.8^{\circ}(\mathrm{c}=0.08$ in MeOH).: Ohsaki, A.; Nagaoka, T.; Yoneda, K.; Kishida, A. Secu'amamine E-G, new alkaloids from Securinega suffruticosa var. amamiensis. Tetrahedron Lett. 2009, 50, 6965-6967.

4. Reaction procedure and spectroscopic data of $\mathbf{S 2}$ was consistent with that reported by the Doris group.: Giovanelli, E.; Moisan, L.; Comesse, S.; Leroux, S.; Rousseau, B.; Hellier, P.; Nicolas, M.; Doris, E. Synthesis of fluorinated catharanthine analogues and investigation of their biomimetic coupling with vindoline. Org. Biomol. Chem. 2013, 11, 5885-5891.

5. Reaction procedure and spectroscopic data of $\mathbf{2 8}$ was consistent with that reported by the Doris group: Giovanelli, E.; Moisan, L.; Comesse, S.; Leroux, S.; Rousseau, B.; Hellier, P.; Nicolas, M.; Doris, E. Synthesis of fluorinated catharanthine analogues and investigation of their biomimetic coupling with vindoline. Org. Biomol. Chem. 2013, 11, 5885-5891.

6. Specific rotation of natural (-)-33 from the isolation report is $[\alpha]^{25} \mathrm{D}:-46^{\circ}\left(c=0.5\right.$ in $\left.\mathrm{CHCl}_{3}\right) .:$ Clivio, P.; Richard, B.; Hadi, H. A.; David, B.; Sevenet, T.; Zeches, M.; Le Men-Olivier, L. Alkaloids from leaves and stem bark of Ervatamia polyneura. Phytochemistry, 1990, 9, 3007-3011.

7. Specific rotation of synthetic (-)-33 from the synthesis report is $[\alpha]^{20} \mathrm{D}:-67^{\circ}\left(c=0.15\right.$ in $\left.\mathrm{CHCl}_{3}\right)$.: Zhou, J.; Tan, D.-X.; Han, F.-S. A divergent enantioselective total synthesis of post-iboga indole alkaloids. Angew. Chem. Int. Ed. 2020, 59, 18731-18740.

8. Specific rotation of natural (-)-35 from the report is $[\alpha]^{25} \mathrm{D}:-27^{\circ} .:$ Danieli, B.; Palmisano, G. Chapter 1 Alkaloids from tabernaemontana. The Alkaloids: Chemistry and Pharmacology, 1986, 27, 1-130.

9. Specific rotation of natural (-)-4 from the isolation report is $[\alpha]^{25} \mathrm{D}:-26^{\circ}\left(C=0.02\right.$ in $\left.\mathrm{CHCl}_{3}\right)$.: Nielsen, H. B.; Hazell, A.; Hazell, R.; Ghia, F.; Torssell, K. B. G. Indole alkaloids and terpenoids fromTabernaemontana markgrafiana. Phytochemistry, 1994, 37, 1729-1735.

10. Specific rotation of synthetic $(-)-4$ from the synthesis report is $[\alpha]^{20} \mathrm{D}:-27.5^{\circ}\left(c=0.2\right.$ in $\left.\mathrm{CHCl}_{3}\right)$.: Zhou, J.; Tan, D.-X.; Han, F.-S. A divergent enantioselective total synthesis of post-iboga indole alkaloids. Angew. Chem. Int. Ed. 2020, 59, 18731-18740.

11. Specific rotation of natural $(+)-5$ from the isolation report is $[\alpha]_{D}:+18^{\circ}\left(c=0.07\right.$ in $\left.\mathrm{CHCl}_{3}\right) .: \mathrm{Kam}$, T.-S.; Sim, L.-M. Dippinines A-D, new iboga-derived indole alkaloids from tabernaemontana. Heterocycles, 2001, 55, 2405-2412.

12. Specific rotation of natural $(+)-6$ from the isolation report is $[\alpha]_{\mathrm{D}}:+231^{\circ}\left(c=0.08\right.$ in $\left.\mathrm{CHCl}_{3}\right) .: \mathrm{Kam}$, T.-S.; Sim, K.-M.; Lim, T.-M. Tronocarpine, a novel pentacyclic indole incorporating a sevenmembered lactam moiety. Tetrahedron Letters, 2000, 41, 2733-2736.

13. Reaction procedure and spectroscopic data of $\mathbf{S 9}$ was consistent with those reported by the Stephenson group: Beatty, J. W.; Stephenson, C. R. J. Synthesis of (-)-Pseudotabersonine, (-)Pseudovincadifformine, and (+)-Coronaridine Enabled by Photoredox Catalysis in Flow. J. Am. Chem. Soc. 2014, 136, 10270-10273. and the Luo group: Zhang, Y.; Xue, Y.; Li, G.; Yuana, H.; Luo, T. Enantioselective synthesis of lboga alkaloids and vinblastine via rearrangements of quaternary ammoniums. Chem. Sci. 2016, 7, 5530-5536.

14. Specific rotation of natural (-)-S11 from the isolation report.) is $[\alpha]_{D}:-8.5^{\circ}(\mathrm{HCl}$ salt, $c=1$ in $\mathrm{MeOH}) .:$ Gorman, M.; Neuss, N.; Cone, N. J.; Deyrup, J. A. Alkaloids from apocynaceae. III. Alkaloids of tabernaemontana and ervatamia. The structure of coronaridine, a new alkaloid related to ibogamine. J. Am. Chem. Soc. 1960, 82, 1142-1145. and synthetic (+)-S11 from the report is [a]D: 
$+6^{\circ}(\mathrm{HCl}$ salt, $C=1$ in $\mathrm{MeOH}) .:$ Beatty, J. W.; Stephenson, C. R. J. Synthesis of (-)Pseudotabersonine, (-)-Pseudovincadifformine, and (+)-Coronaridine Enabled by Photoredox Catalysis in Flow. J. Am. Chem. Soc. 2014, 136, 10270-10273.

15. Specific rotation of natural (-)-44 from the isolation report are $[\alpha]_{\mathrm{D}}:-41 \pm 2^{\circ}\left(c=1.55\right.$ in $\left.\mathrm{CHCl}_{3}\right)$ and [a] $]_{D}:-49 \pm 8^{\circ}\left(c=0.065\right.$ in $\left.\mathrm{CHCl}_{3}\right)$.: Agwada, V. C.; Morita, Y.; Renner, U.; Hesse, M.; Schmid, H. Die alkaloide von Gabunia eglandulosa STAPF 155. Mitteilung über alkaloide. Helv. Chim. Acta. 1975, 58, 1001-1016.

16. Reaction procedure and spectroscopic data of $\mathbf{S} 15$ was consistent with those reported by the Cook group: Yu, J.; Wang, T.; Liu, X.; Deschamps, J.; Flippen-Anderson, J.; Liao, X.; Cook, J. M. General approach for the synthesis of sarpagine indole alkaloids. Enantiospecific total synthesis of (+)-vellosimine, (+)-normacusine B, (-)-alkaloids $\mathrm{Q}_{3},(-)$-panarine, (+)- $\mathrm{Na}$-methylvellosimine, and (+)$\mathrm{Na}_{\mathrm{a}}$-methyl-16-epipericyclivine. J .Org. Chem. 2003, 68, 7565-7581.

17. Specific rotation of synthetic (+)-7 from the report is $[\alpha]_{D}:+4.6^{\circ}(c=1$ in MeOH).: Yu, J.; Wang, T.; Liu, X.; Deschamps, J.; Flippen-Anderson, J.; Liao, X.; Cook, J. M. General approach for the synthesis of sarpagine indole alkaloids. Enantiospecific total synthesis of (+)-vellosimine, $(+)-$ normacusine $\mathrm{B},(-)$-alkaloids $\mathrm{Q}_{3},(-)$-panarine, (+)- $\mathrm{N}_{\mathrm{a}}$-methylvellosimine, and (+)- $\mathrm{N}_{\mathrm{a}}$-methyl-16epipericyclivine. J .Org. Chem. 2003, 68, 7565-7581. 


\section{Copies of ${ }^{1} \mathrm{H}$ NMR and ${ }^{13} \mathrm{C}$ NMR Spectra}


Parameter

\section{Origin}

2 Instrument

3 Solvent

4 Temperature

5 Experiment

6 Probe

7 Number of Scans

8 Spectrometer Frequency 400.13

9 Nucleus

spect

CDCl3

298.0

10

4

$1 \mathrm{H}$
Value

Bruker BioSpin GmbH

Z108618_0389 (PA BBO 400S1 BBF-H-D-05 Z PLUS)

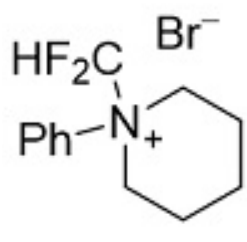

11

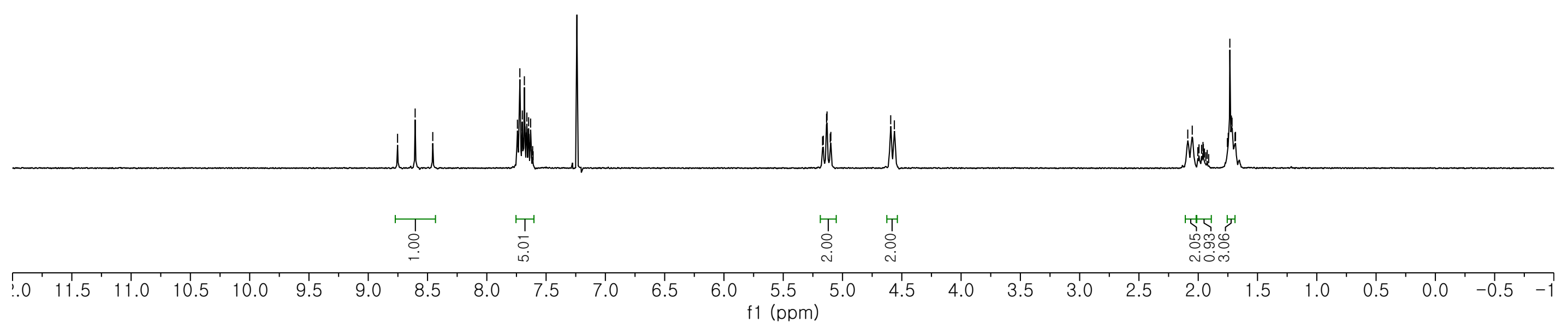


Parameter

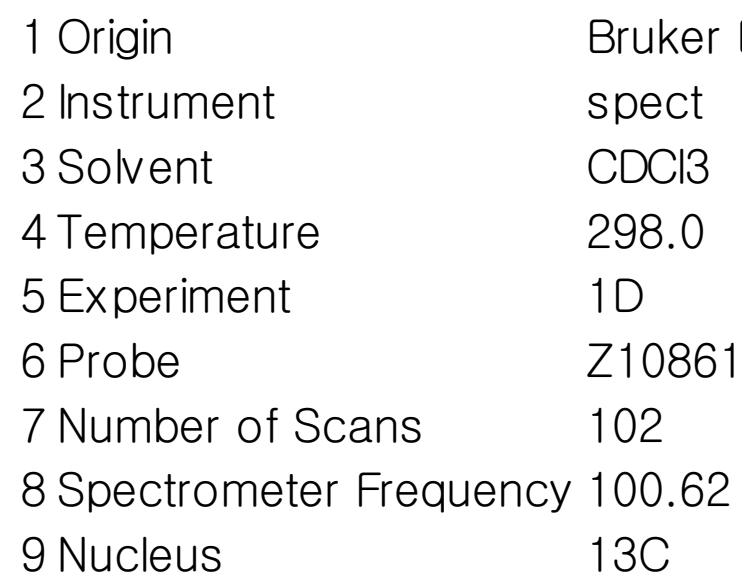

Value

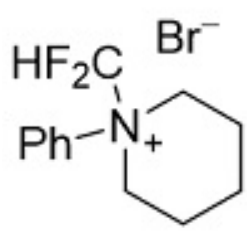

11

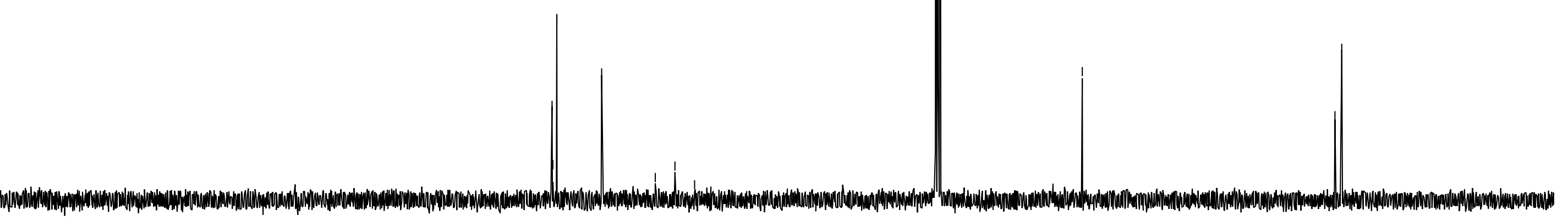




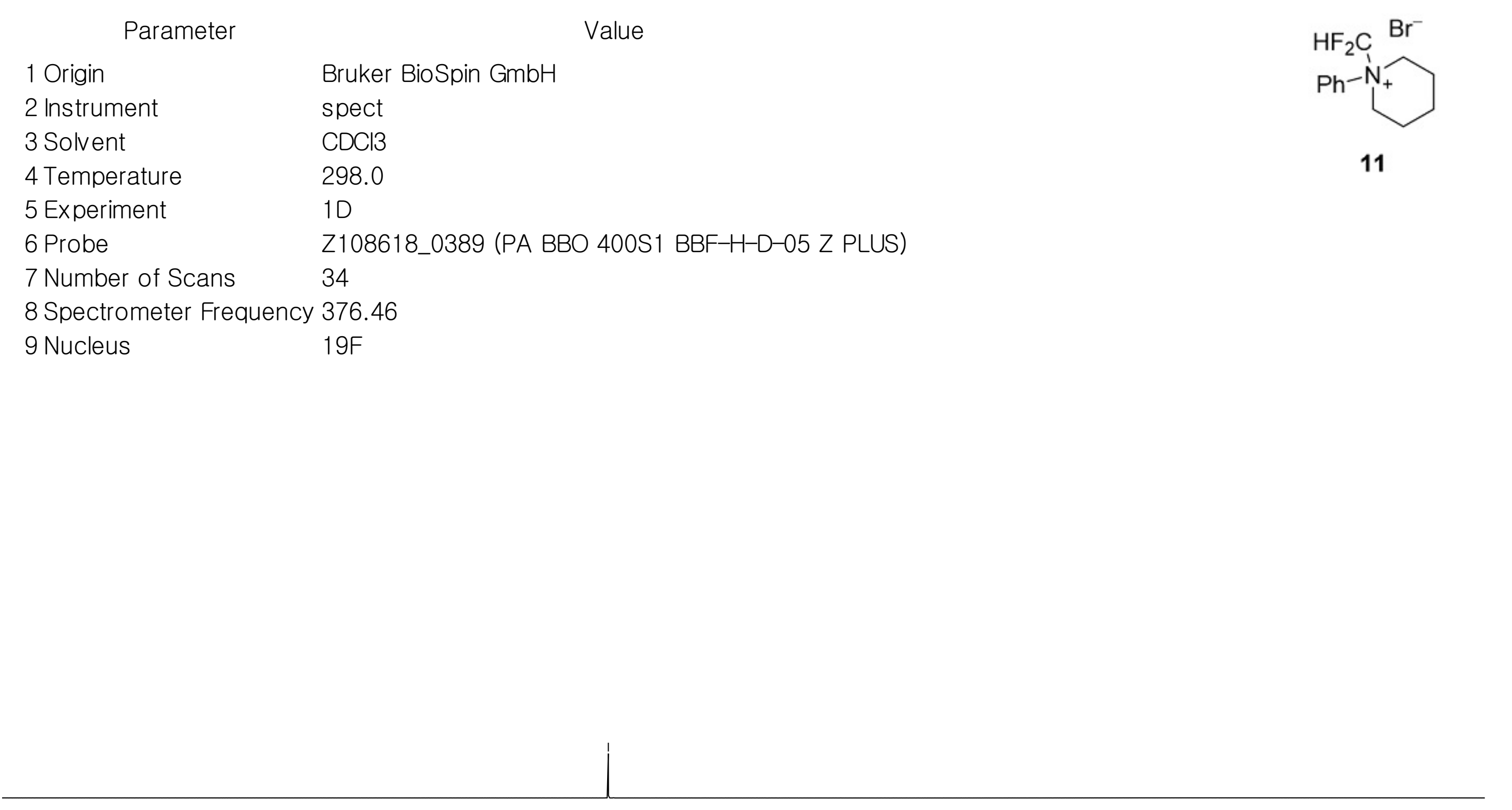




\section{Parameter}

\section{Origin}

2 Instrument

3 Solvent

4 Temperature

5 Experiment

6 Probe

7 Number of Scans

8 Spectrometer Frequency 500.23

9 Nucleus

$\mathrm{CDCl} 3$

298.0

10

5

$1 \mathrm{H}$
Value

Bruker BioSpin GmbH

Avance

Z151574_0070 (PI HR-BBO500S1-BBF/ H/ D-5.0-Z SP)

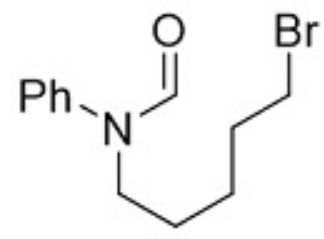

13

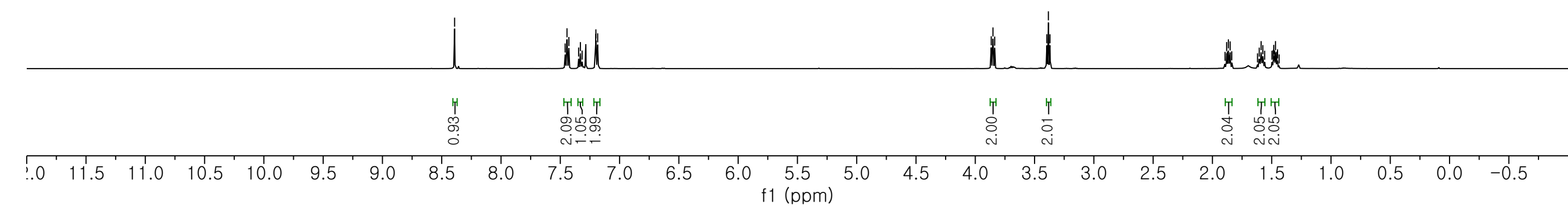




\section{Parameter}

Value

\section{Origin}

2 Instrument

3 Solvent

4 Temperature

5 Experiment

6 Probe

7 Number of Scans

8 Spectrometer Frequency 150.86

9 Nucleus

$13 \mathrm{C}$
Varian

vnmrs

cdcl3

25.0

10

5mm_One_probe

30 


\section{Parameter}

\section{Value}

1 Origin

2 Instrument

3 Solvent

4 Temperature

5 Experiment

6 Probe

7 Number of Scans

8 Spectrometer Frequency 400.12

9 Nucleus

$1 \mathrm{H}$
Bruker BioSpin GmbH

spect

$\mathrm{CDCl} 3$

298.3

10

Z116098_0402 (PA BBO 400S1 BBF-H-D-05 Z SP)
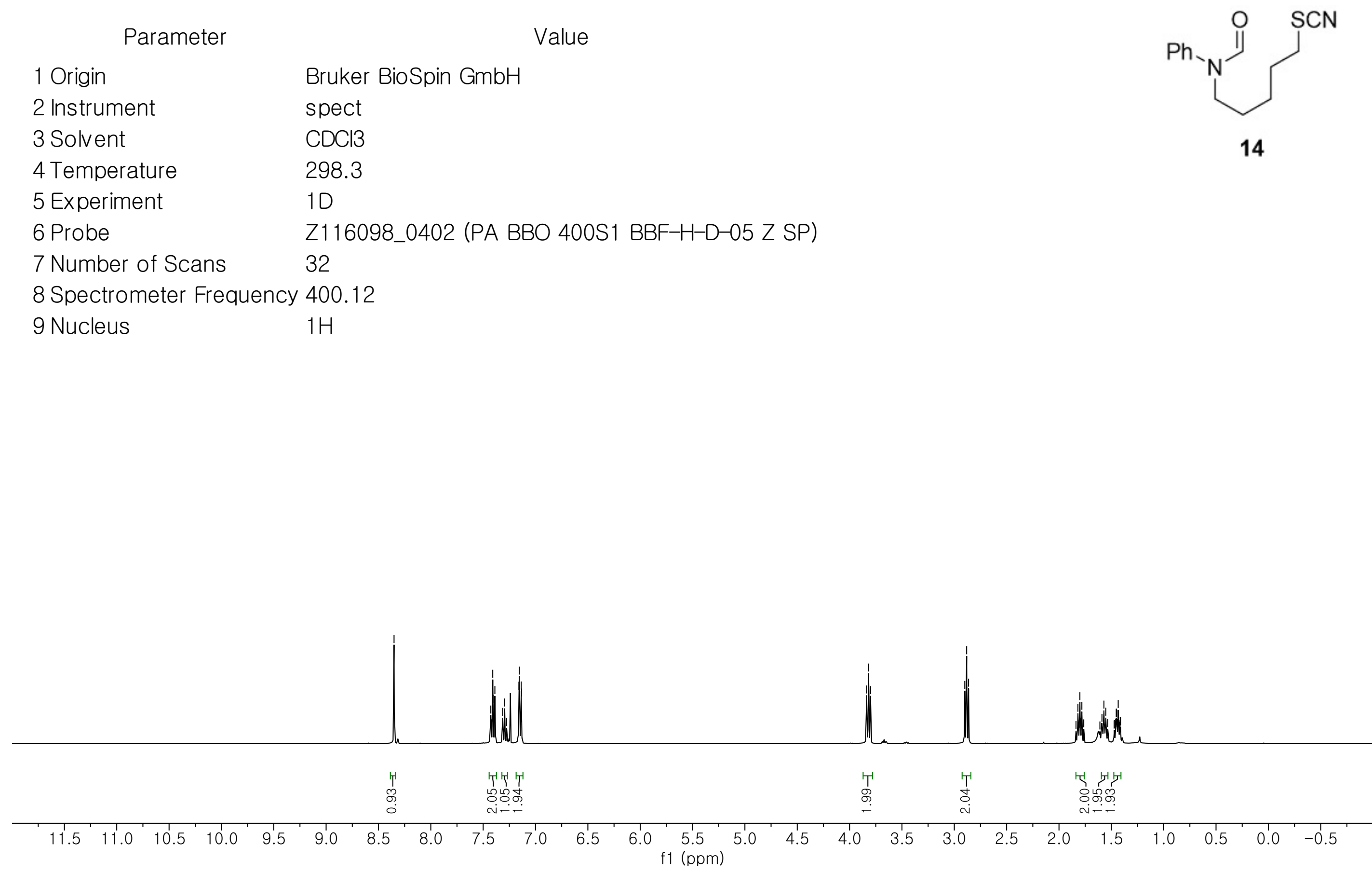


\section{Parameter}

Value
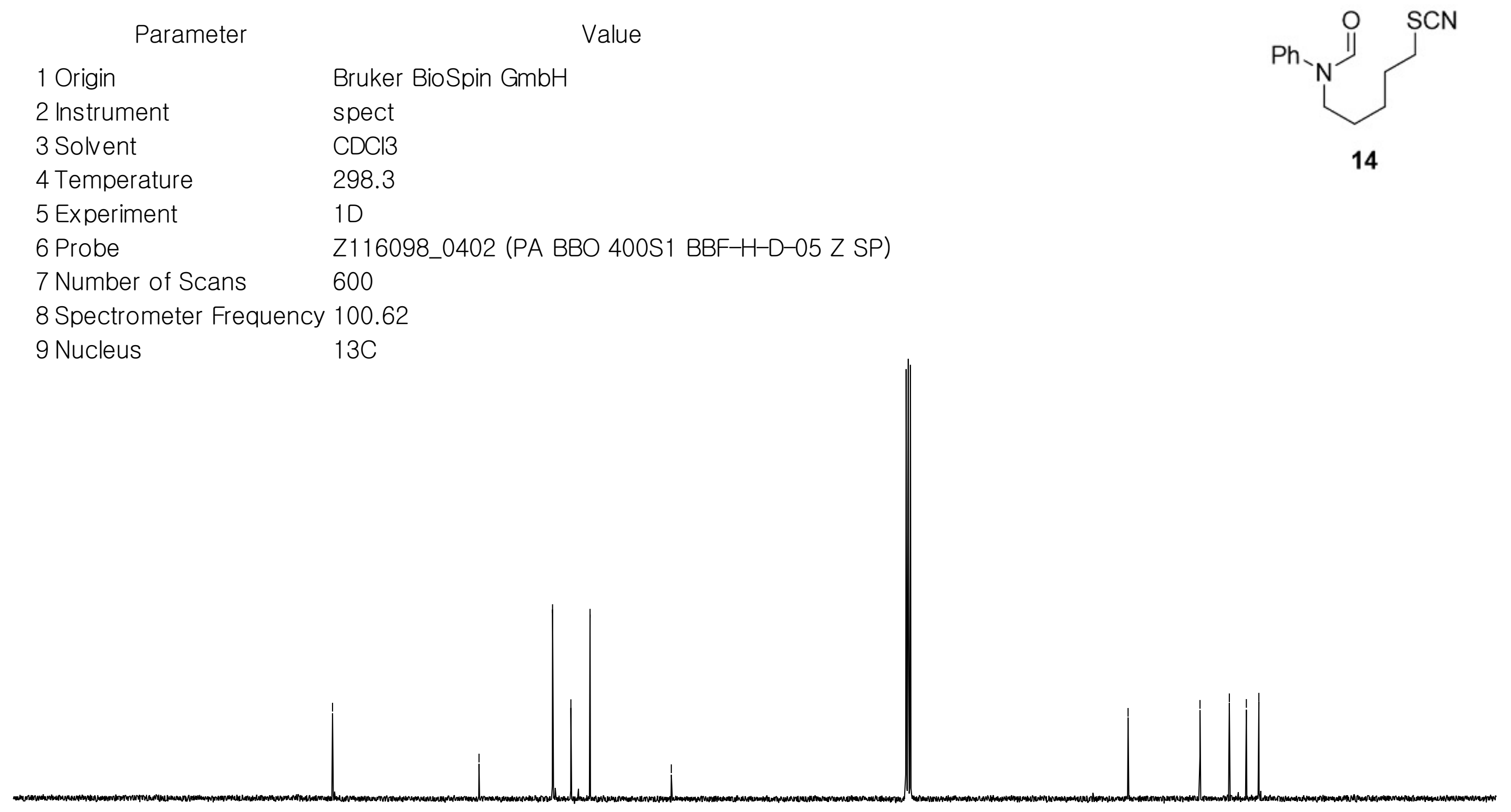

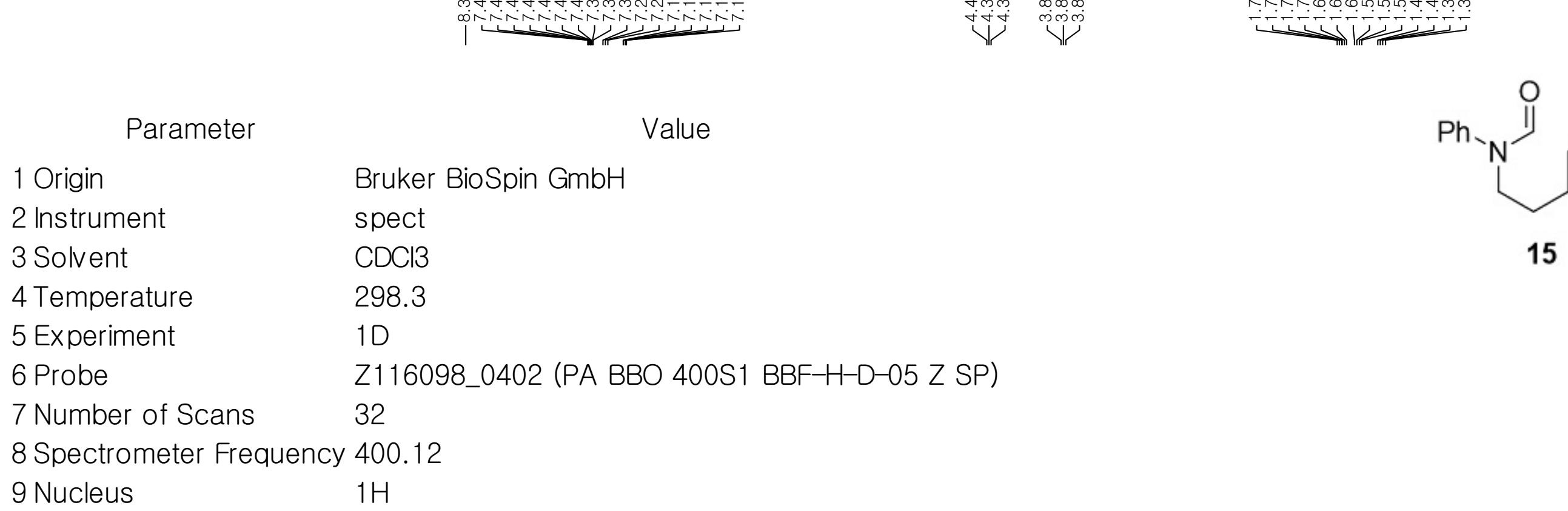

1 Origin

2 Instrument

3 Solvent

4 Temperature

5 Experiment

6 Probe

7 Number of Scans

8 Spectrometer Frequency 400.12

9 Nucleus

Bruker BioSpin GmbH

spect

$\mathrm{CDCl} 3$

298.3

$1 \mathrm{D}$

Z116098_0402 (PA BBO 400S1 BBF-H-D-05 Z SP)

32

15

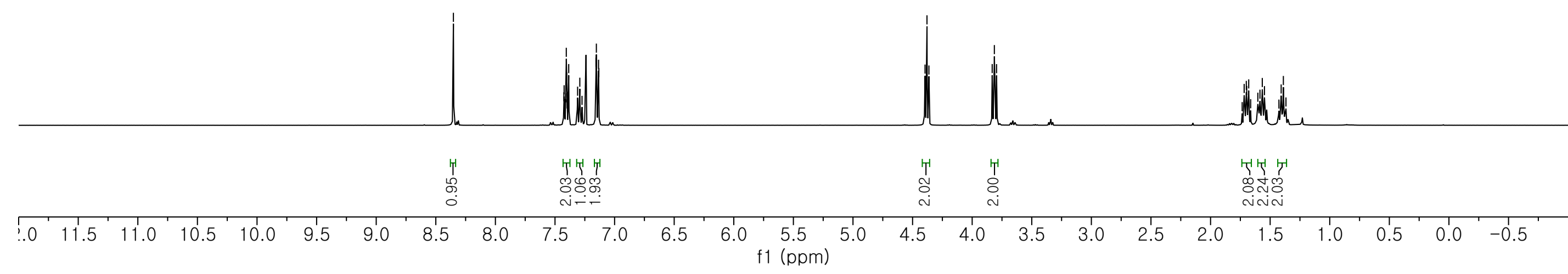


Bio-Patterned Reorganization of Alkaloids Enabled by Ring-Opening Functionalization of Tertiary Amines

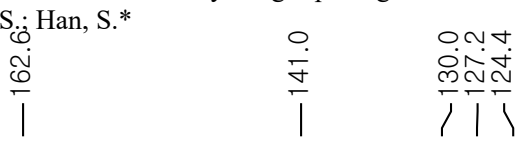

\section{Parameter}

\section{Origin}

2 Instrument

3 Solvent

4 Temperature

5 Experiment

6 Probe

7 Number of Scans

8 Spectrometer Frequency 100.62

9 Nucleus

spect

$\mathrm{CDCl} 3$

298.3

10 696

$13 \mathrm{C}$
Value

Bruker BioSpin GmbH

Z116098_0402 (PA BBO 400S1 BBF-H-D-05 Z SP) 


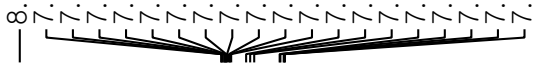

\section{Parameter}

1 Origin

2 Instrument

3 Solvent

4 Temperature

5 Experiment

6 Probe

7 Number of Scans

8 Spectrometer Frequency 400.12

9 Nucleus

spect

$\mathrm{CDCl} 3$

298.3

10

32

$1 \mathrm{H}$

\section{Value}

Bruker BioSpin GmbH

Z116098_0402 (PA BBO 400S1 BBF-H-D-05 Z SP)

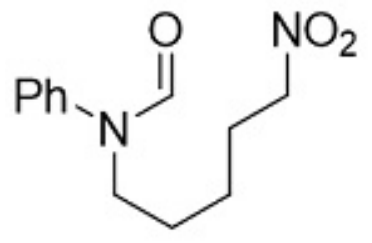

16

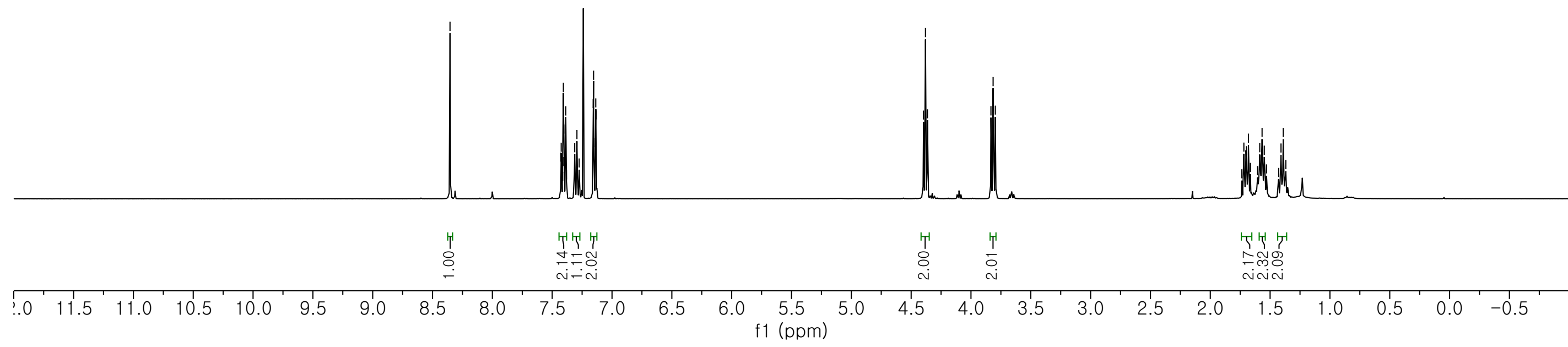


of Alkaloids Enabled by Ring-Opening Functionalization of Tertiary Amines

il

\section{Parameter}

Value

1 Origin

Bruker BioSpin GmbH

2 Instrument

spect

3 Solvent

$\mathrm{CDCl} 3$

298.3

4 Temperature

10

\section{Probe}

7 Number of Scans

Z116098_0402 (PA BBO 400S1 BBF-H-D-05 Z SP) 1024

8 Spectrometer Frequency 100.62

9 Nucleus

$13 \mathrm{C}$ 


\section{Parameter}

Value

1 Origin

2 Instrument

3 Solvent

4 Temperature

5 Experiment

6 Probe

7 Number of Scans

8 Spectrometer Frequency 400.12

9 Nucleus

spect

$\mathrm{CDCl} 3$

298.3

10

32

$1 \mathrm{H}$
Bruker BioSpin GmbH

Z116098_0402 (PA BBO 400S1 BBF-H-D-05 Z SP)<smiles>CC(=O)OCCCCCN(C=O)c1ccccc1</smiles>

17

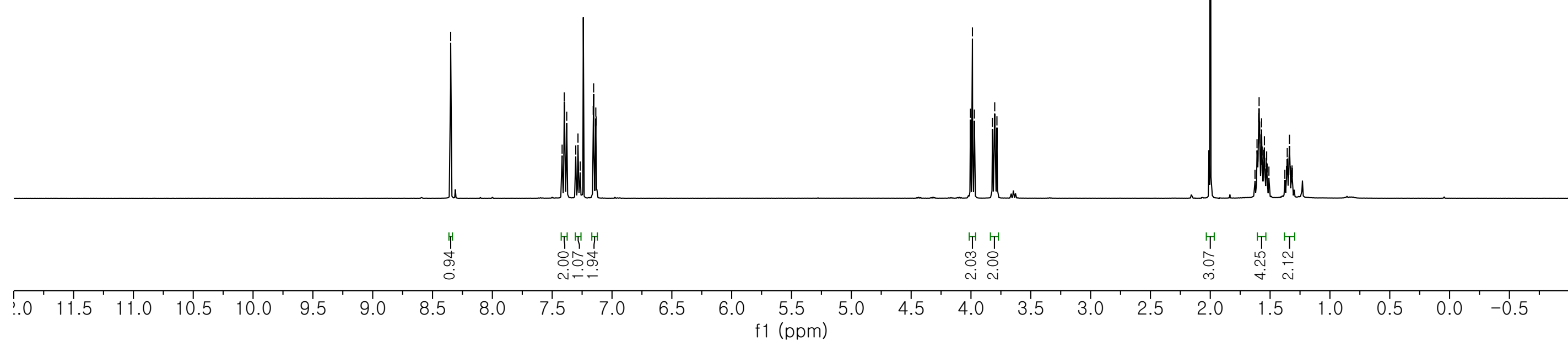




\section{Parameter}

1 Origin

2 Instrument

3 Solvent

4 Temperature

5 Experiment

6 Probe

7 Number of Scans

Bruker BioSpin GmbH

spect

$\mathrm{CDCl} 3$

298.3

10

Z116098_0402 (PA BBO 400S1 BBF-H-D-05 Z SP) 1000

8 Spectrometer Frequency 100.62

9 Nucleus

$13 \mathrm{C}$

\section{Value}

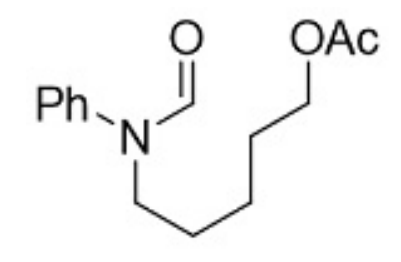

17

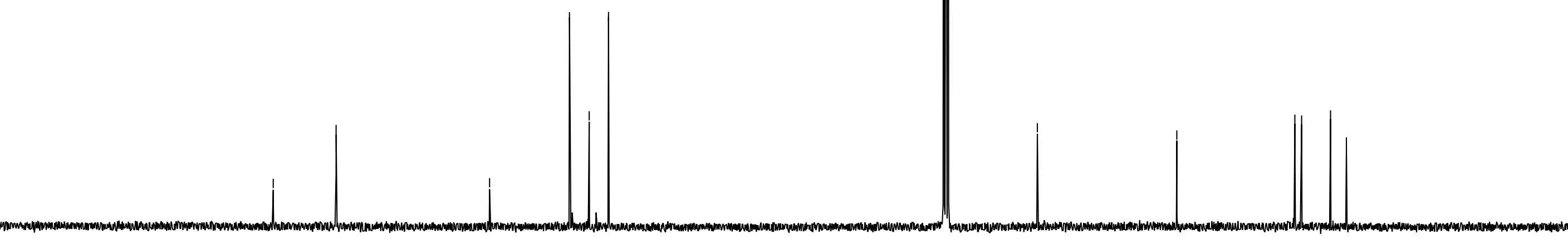




\begin{tabular}{ll}
\multicolumn{1}{c}{ Parameter } & \multicolumn{1}{c}{ Value } \\
1 Origin & Varian \\
2 Instrument & vnmrs \\
3 Solvent & cdcl3 \\
4 Temperature & 25.0 \\
5 Experiment & 10 \\
6 Probe & $5 \mathrm{~mm}$ OOne_probe \\
7 Number of Scans & 16 \\
8 Spectrometer Frequency 599.93 \\
9 Nucleus & $1 \mathrm{H}$
\end{tabular}

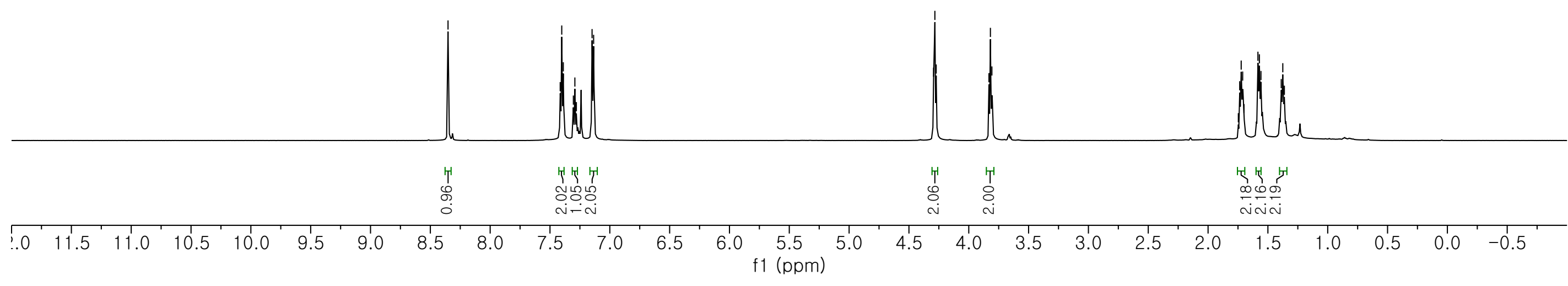




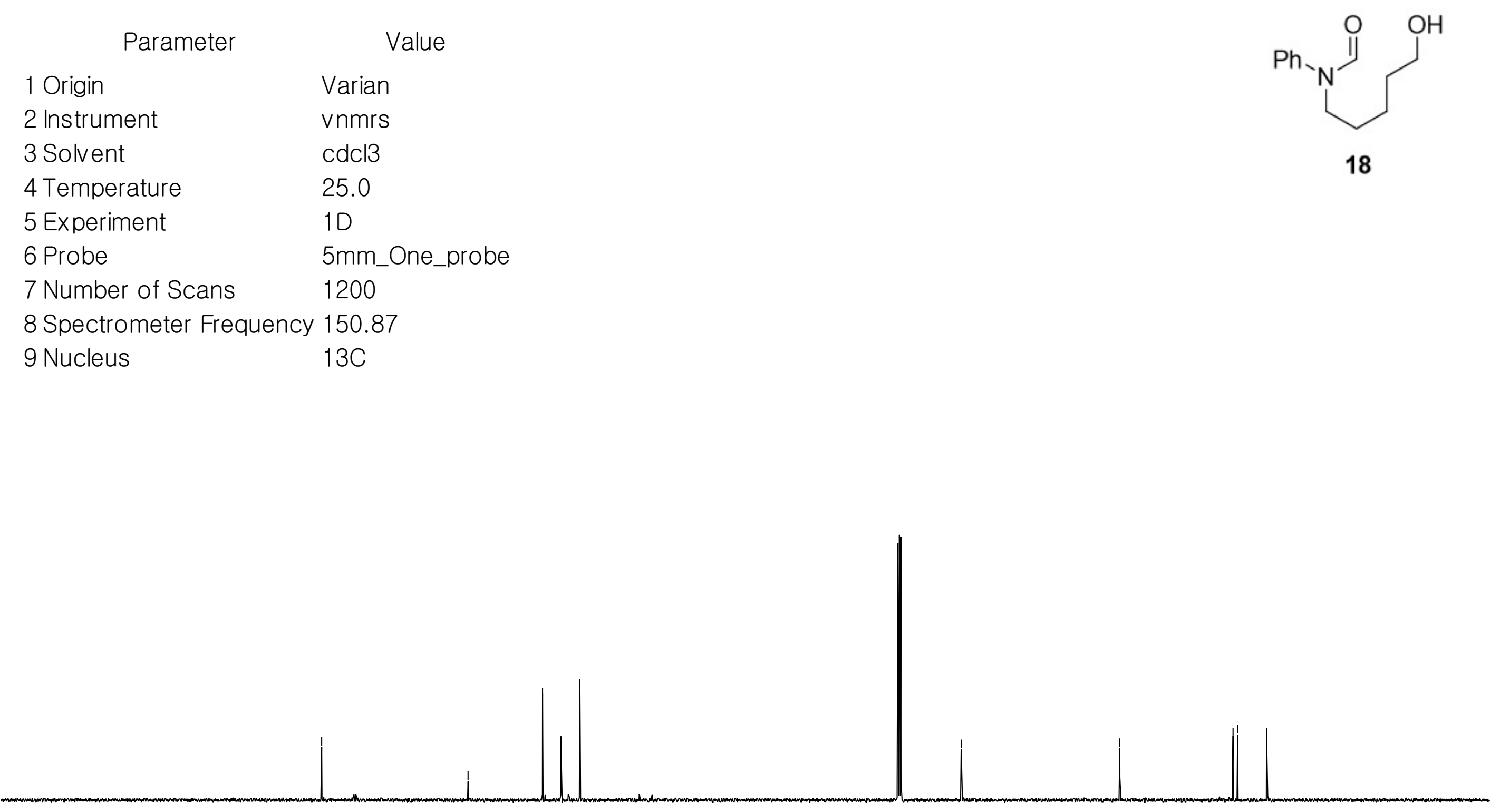




\section{Parameter}

1 Origin

2 Instrument

3 Solvent

4 Temperature

5 Experiment

6 Probe

7 Number of Scans

8 Spectrometer Frequency 400.12

9 Nucleus

spect

$\mathrm{CDCl} 3$

298.3

10

32
Value

Bruker BioSpin GmbH

Z116098_0402 (PA BBO 400S1 BBF-H-D-05 Z SP)

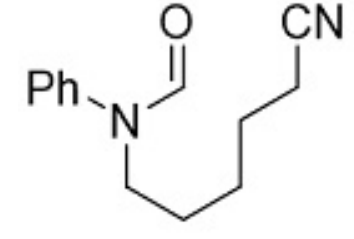

19

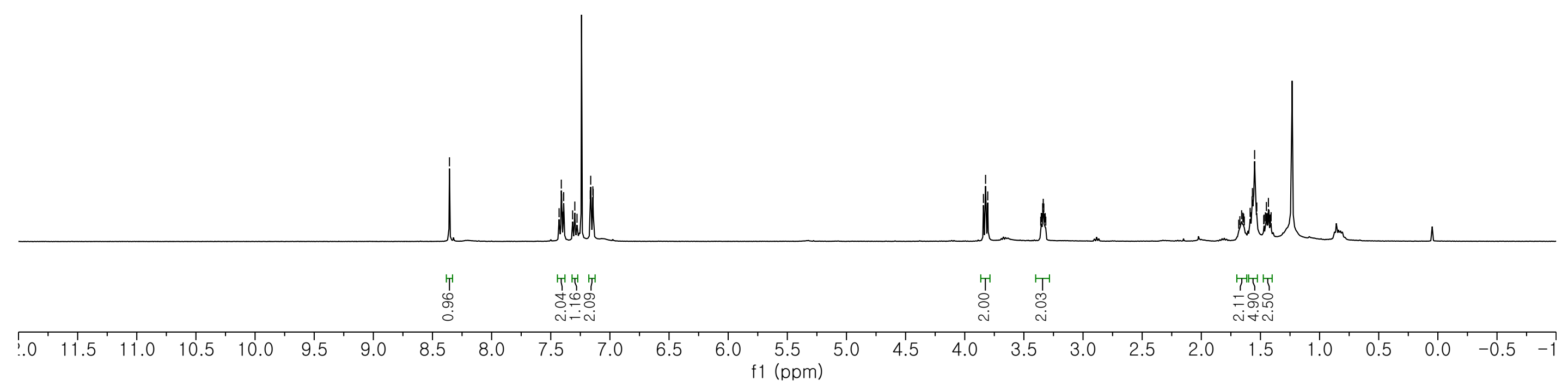




\section{Parameter}

1 Origin

2 Instrument

3 Solvent

4 Temperature

5 Experiment

6 Probe

7 Number of Scans

8 Spectrometer Frequency 100.62

9 Nucleus

$13 \mathrm{C}$

spect

CDCl3

298.3

10 4800

\section{Value}

Bruker BioSpin $\mathrm{GmbH}$

Z116098_0402 (PA BBO 400S1 BBF-H-D-05 Z SP) 

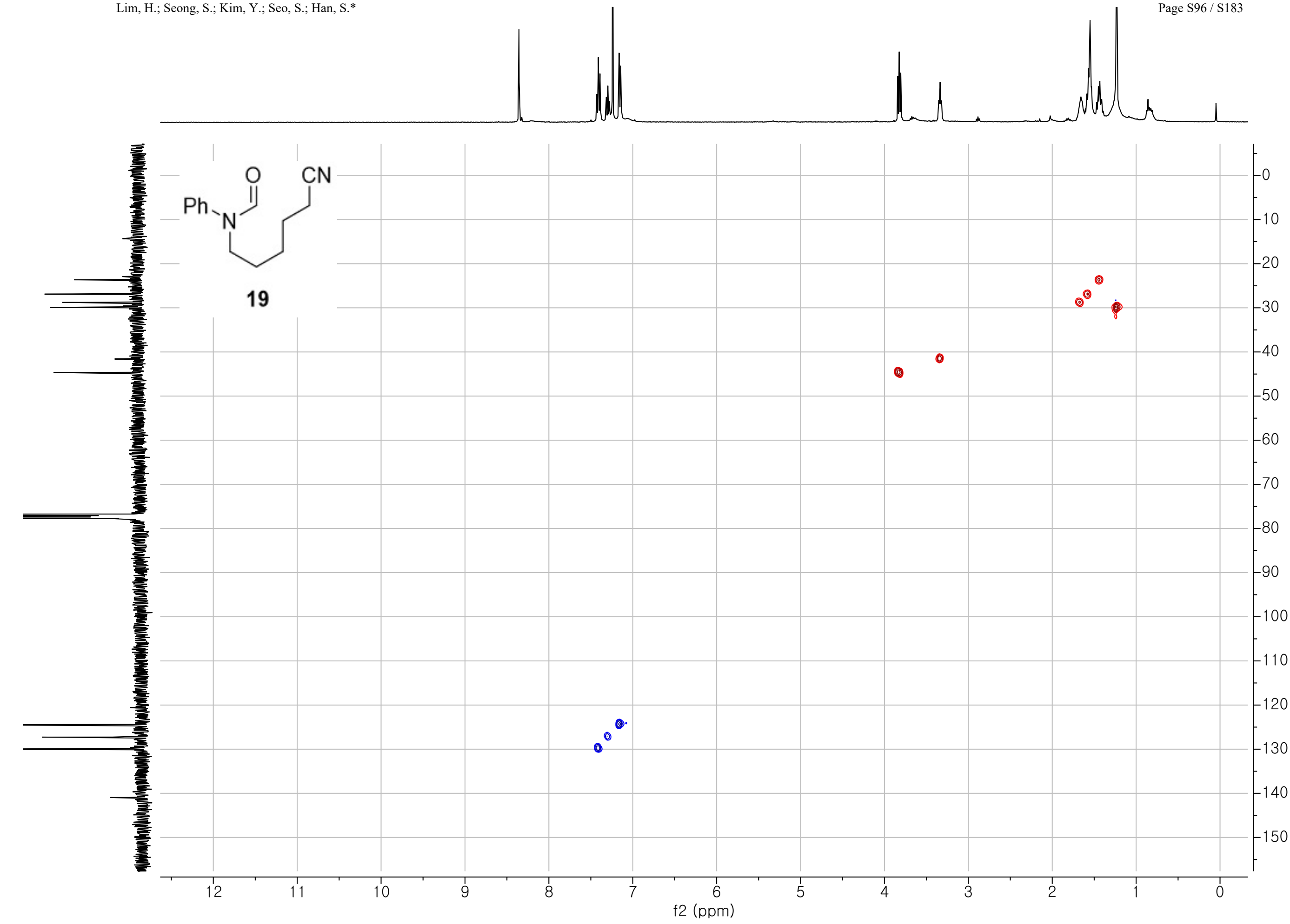


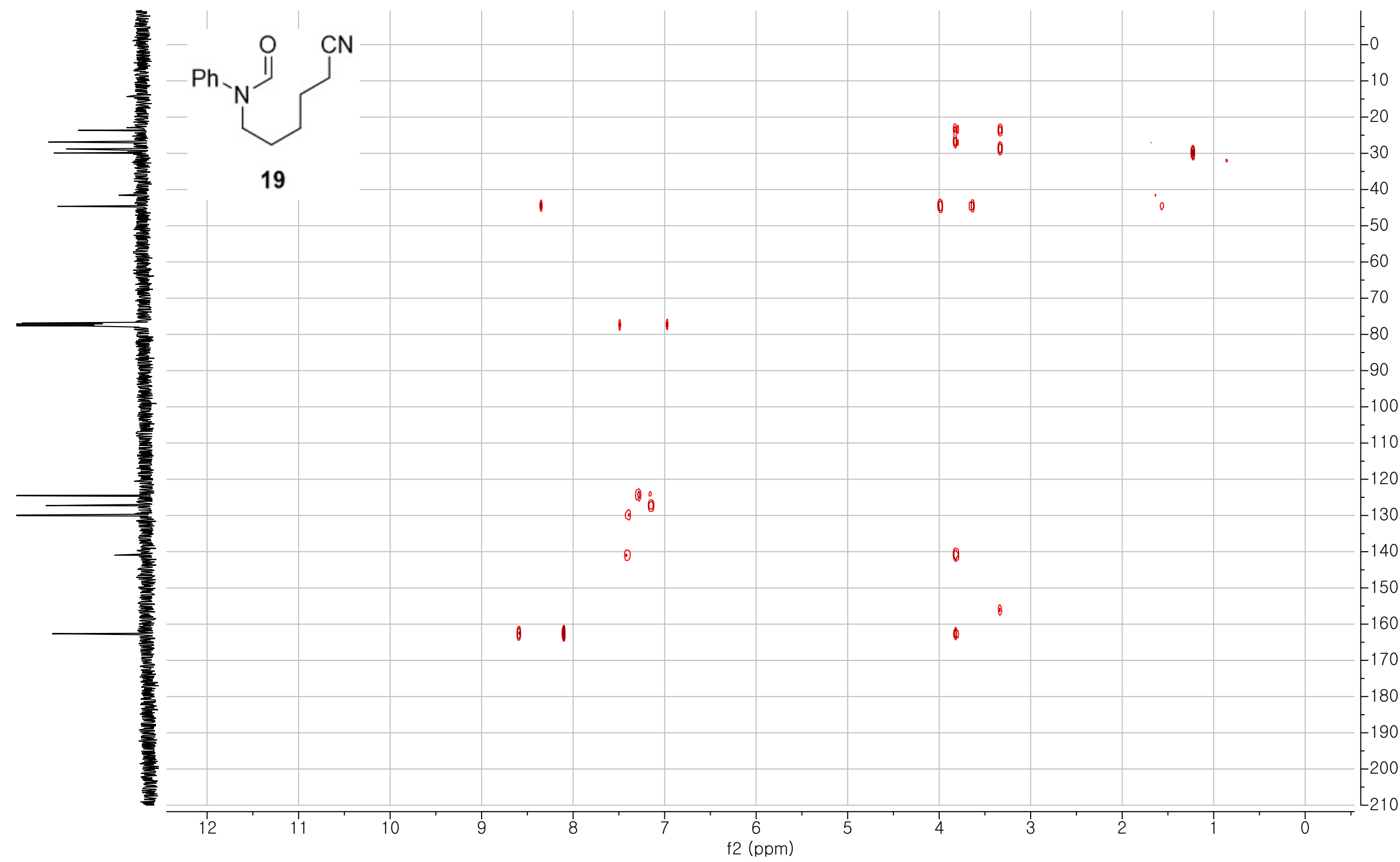




\section{Parameter}

Value

\section{Origin}

2 Instrument

3 Solvent

4 Temperature

5 Experiment

6 Probe

7 Number of Scans

8 Spectrometer Frequency 500.23

9 Nucleus

$\mathrm{CDCl} 3$

298.0

10

15

$1 \mathrm{H}$
Bruker BioSpin GmbH

Avance

Z151574_0070 (PI HR-BBO500S1-BBF/ H/ D-5.0-Z SP)

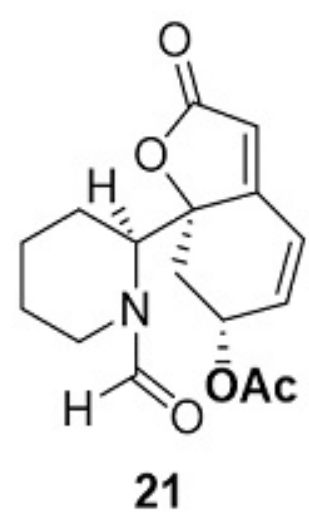

21

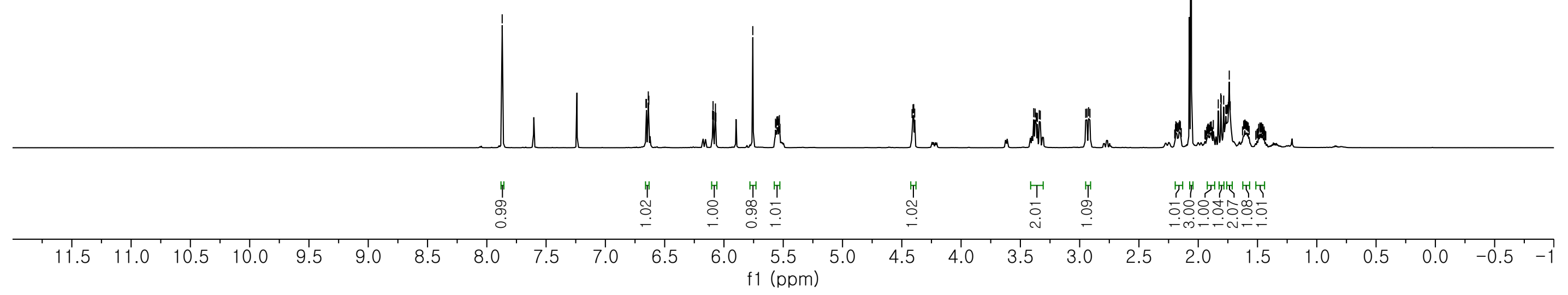




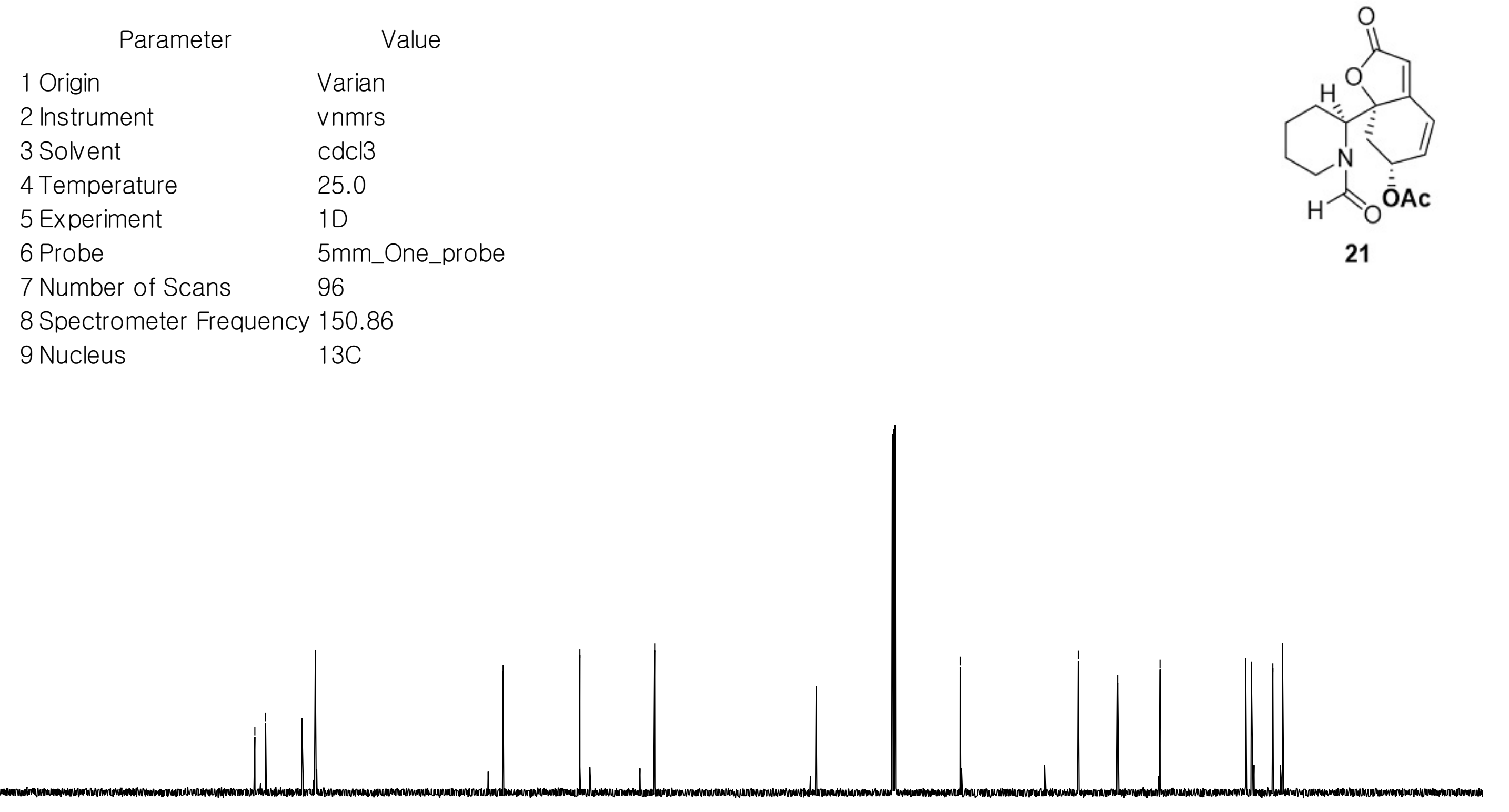


Parameter

Value

1 Origin

2 Instrument

3 Solvent

4 Temperature

5 Experiment

6 Probe

7 Number of Scans

8 Spectrometer Frequency 500.23

9 Nucleus

$\mathrm{CDCl} 3$

298.0

10

15

$1 \mathrm{H}$
Bruker BioSpin GmbH

Avance

Z151574_0070 (PI HR-BBO500S1-BBF/ H/ D-5.0-Z SP)

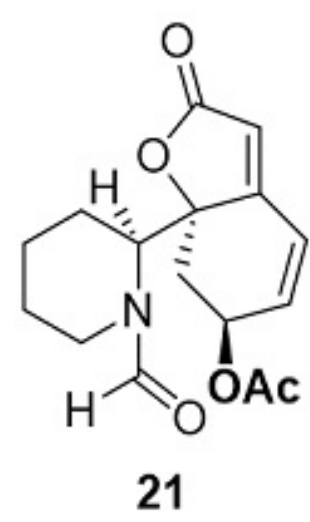

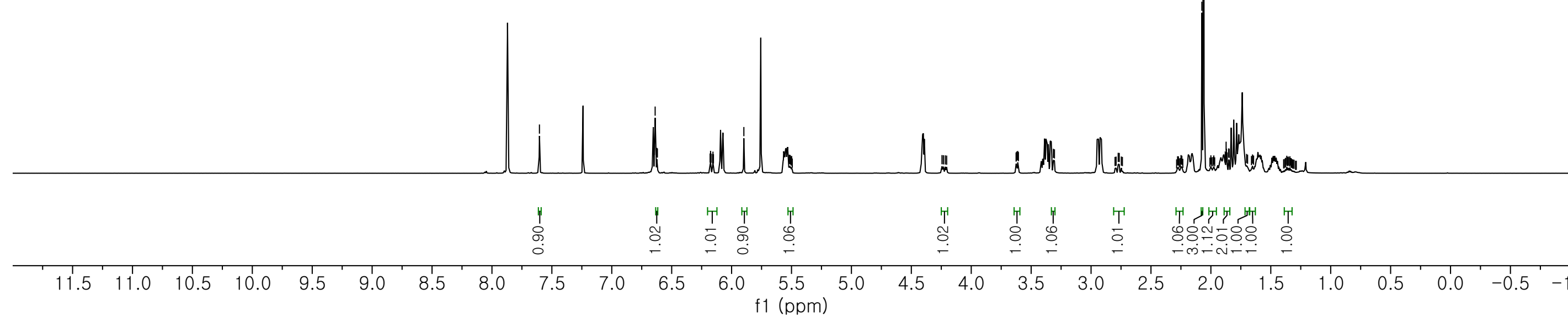




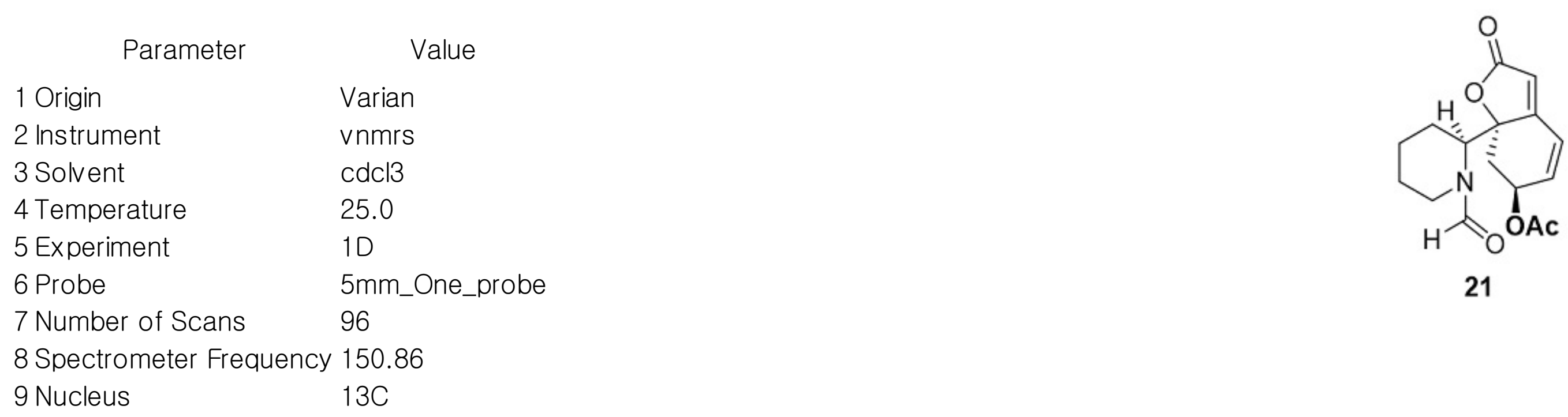




\section{Parameter}

\section{Value}

1 Origin

2 Instrument

3 Solvent

4 Temperature

5 Experiment

6 Probe

7 Number of Scans

8 Spectrometer Frequency 500.23

9 Nucleus

$\mathrm{CDCl} 3$

298.0

10

9

$1 \mathrm{H}$
Bruker BioSpin GmbH

Avance

Z151574_0070 (PI HR-BBO500S1-BBF/ H/ D-5.0-Z SP)

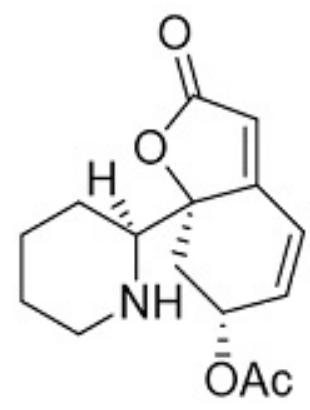

S1

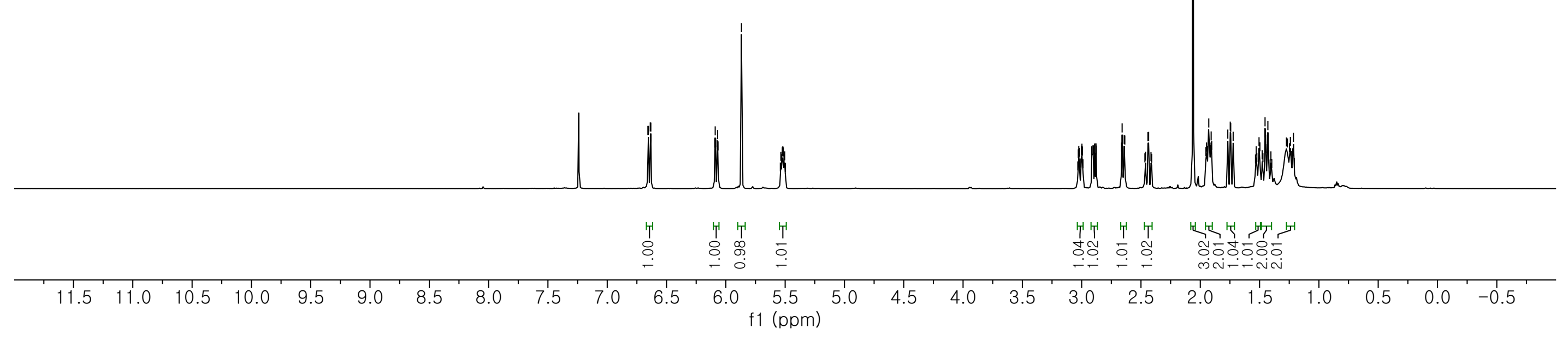


Bio-Patterned Reorganization of Alkaloids Enabled by Ring-Opening Functionalization of Tertiary Amines

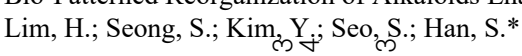
।

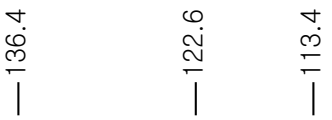

\begin{tabular}{ll}
\multicolumn{1}{c}{ Parameter } & \multicolumn{1}{c}{ Value } \\
1 Origin & Varian \\
2 Instrument & vnmrs \\
3 Solvent & cdcl3 \\
4 Temperature & 25.0 \\
5 Experiment & 10 \\
6 Probe & 5 mm_One_probe \\
7 Number of Scans & 262 \\
8 Spectrometer Frequency & 150.86 \\
9 Nucleus & $13 \mathrm{C}$
\end{tabular}

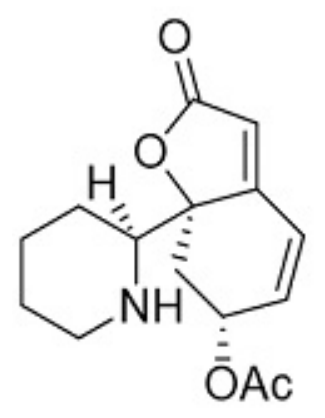

S1

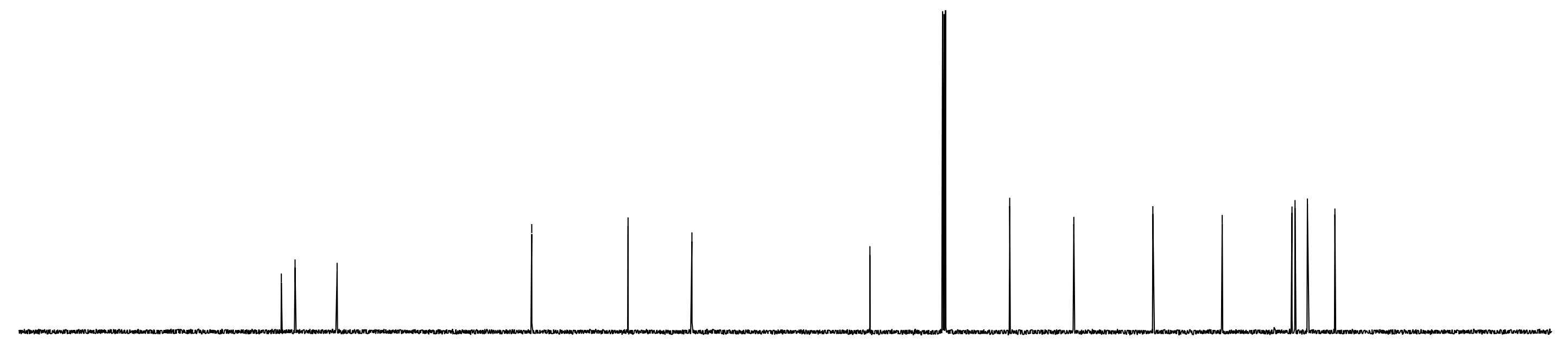




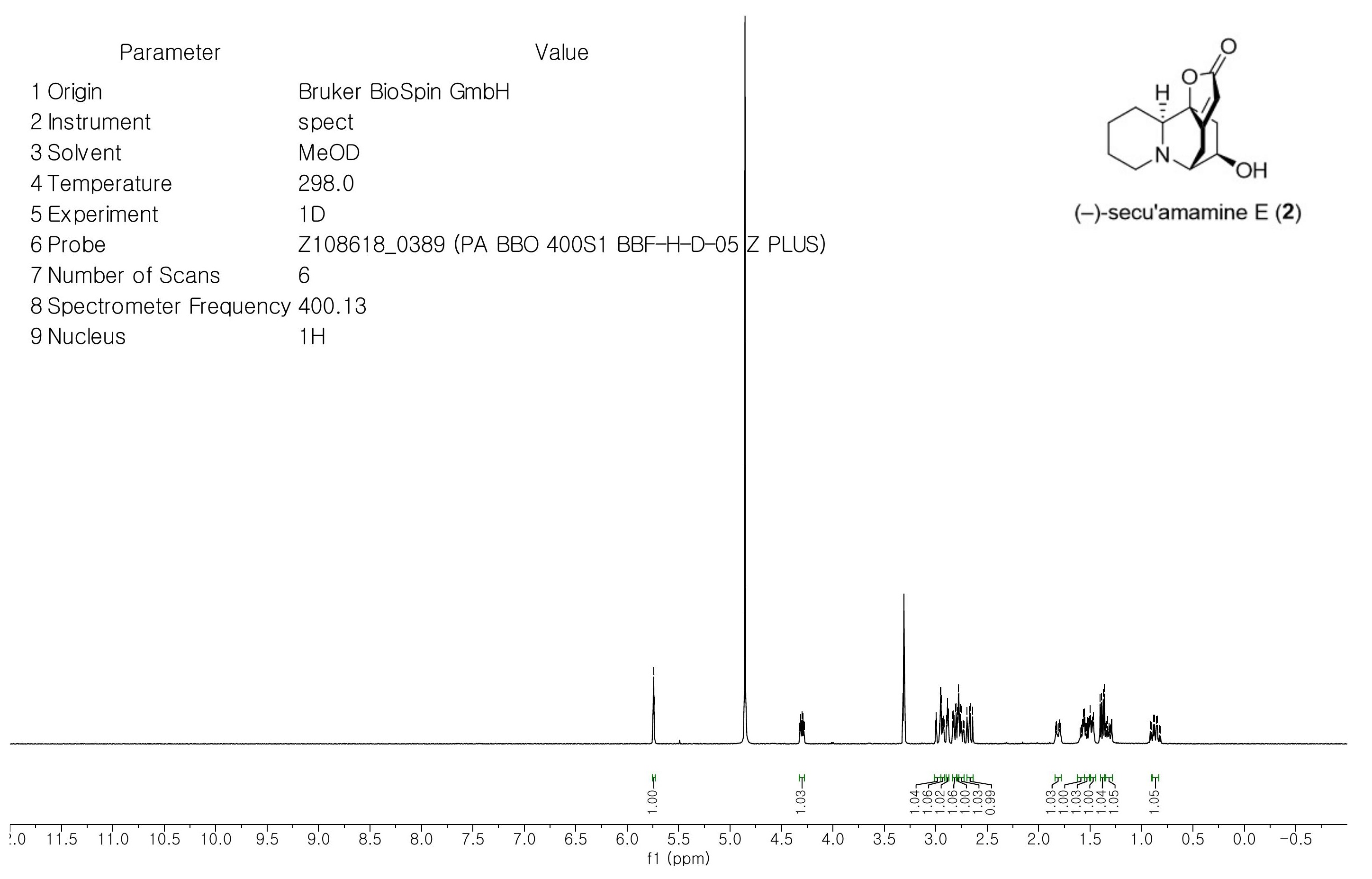




\section{Parameter}

Value

1 Origin

Varian

2 Instrument

vnmrs

cd3od

25.0

4 Temperature

10

$5 \mathrm{~mm} \_$One_probe

108

7 Number of Scans

8 Spectrometer Frequency 150.86

9 Nucleus

$13 \mathrm{C}$

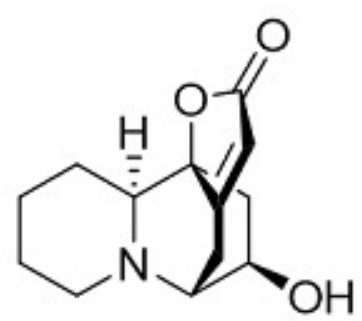

(-)-secu'amamine E (2)

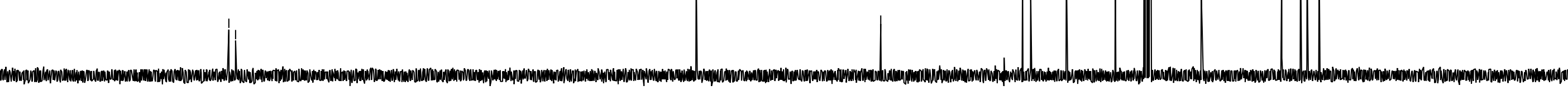




\section{Parameter}

1 Origin

2 Instrument

3 Solvent

4 Temperature

5 Experiment

6 Probe

7 Number of Scans

8 Spectrometer Frequency 500.23

9 Nucleus

$\mathrm{CDCl} 3$

298.0

10 38

$1 \mathrm{H}$
Value

Bruker BioSpin $\mathrm{GmbH}$

Avance

Z151574_0070 (PI HR-BBO500S1-BBF/ H/ D-5.0-Z SP)

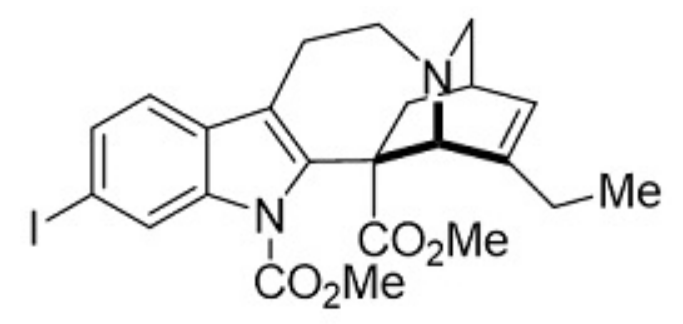

22 


\section{Parameter}

\section{Origin}

2 Instrument

3 Solvent

4 Temperature

5 Experiment

6 Probe

7 Number of Scans

8 Spectrometer Frequency 100.62

9 Nucleus

spect

$\mathrm{CDCl} 3$

298.0

10 4000

$13 \mathrm{C}$
Value

Bruker BioSpin GmbH

Z116098_0402 (PA BBO 400S1 BBF-H-D-05 Z SP)

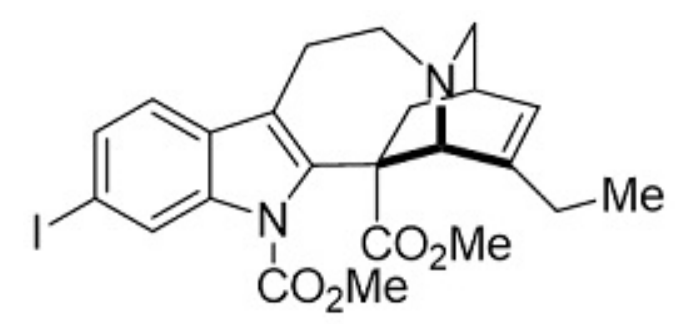

22

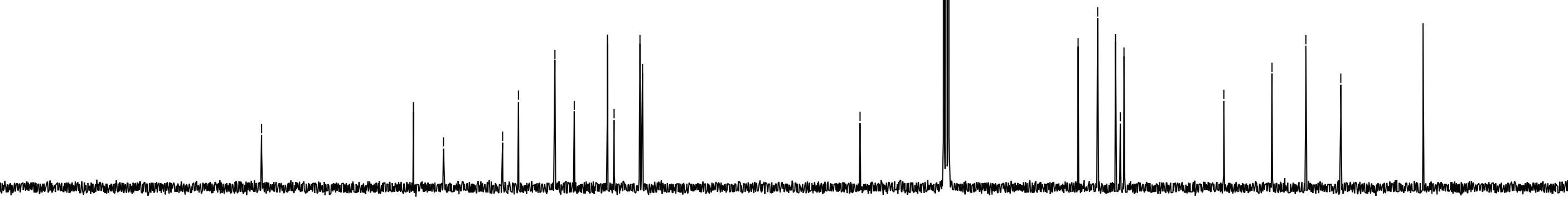


Parameter

\section{Origin}

2 Instrument

3 Solvent

4 Temperature

5 Experiment

6 Probe

7 Number of Scans

8 Spectrometer Frequency 400.13

9 Nucleus

spect

$\mathrm{CDCl} 3$

298.0

10 6

$1 \mathrm{H}$

\section{Value}

Bruker BioSpin GmbH

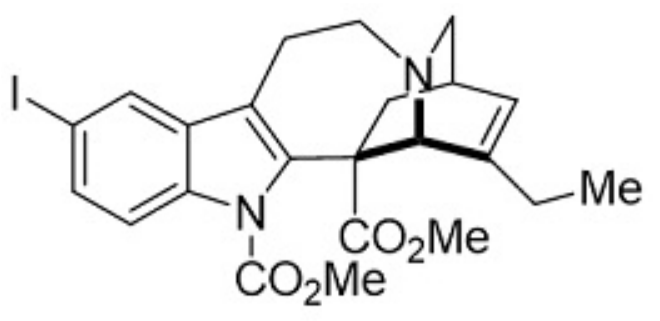

S3

Z108618_0389 (PA BBO 400S1 BBF-H-D-05 Z PLUS)

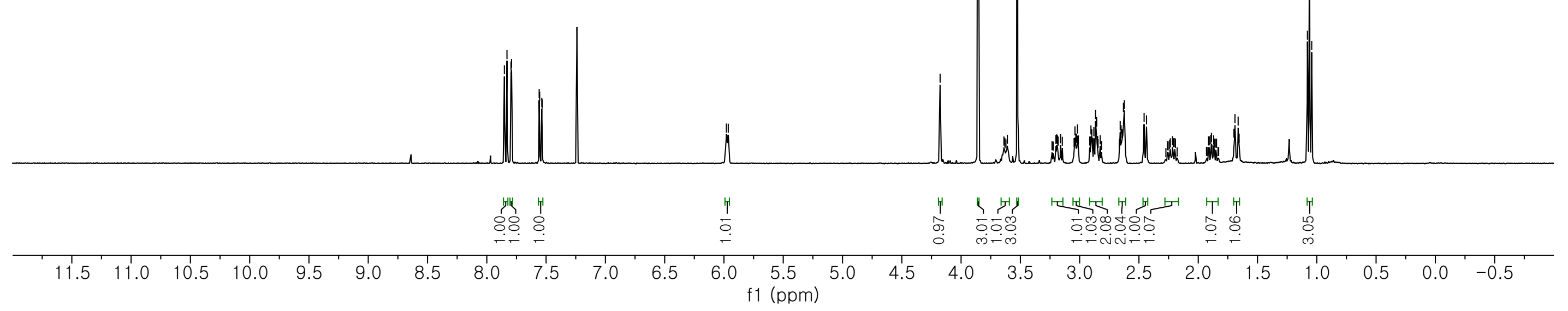




\section{Parameter}

Value

1 Origin

2 Instrument

3 Solvent

4 Temperature

5 Experiment

6 Probe

7 Number of Scans

8 Spectrometer Frequency 100.62

9 Nucleus
Bruker BioSpin GmbH

spect

CDCl3

298.0

10

Z108618_0389 (PA BBO 400S1 BBF-H-D-05 Z PLUS) 779

$13 \mathrm{C}$

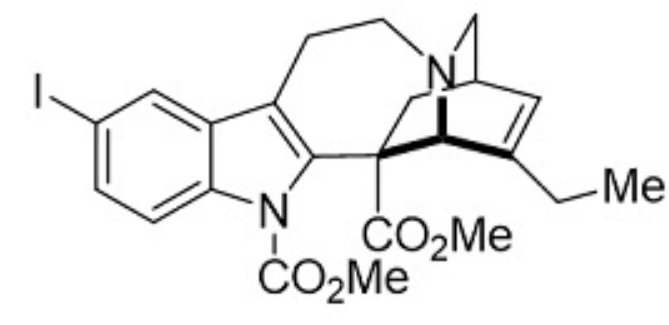

S3

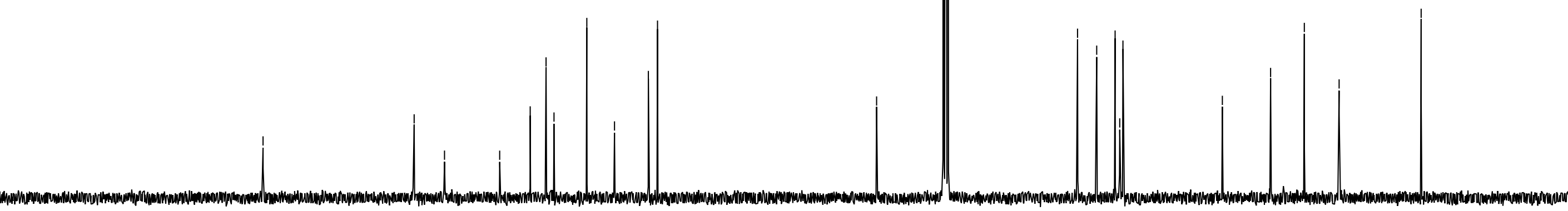


Parameter

1 Origin

2 Instrument

3 Solvent

4 Temperature

5 Experiment

6 Probe

7 Number of Scans

8 Spectrometer Frequency 500.23

9 Nucleus

$\mathrm{CDCl} 3$

298.0

10

8

$1 \mathrm{H}$
Value

Bruker BioSpin $\mathrm{GmbH}$

Avance

Z151574_0070 (PI HR-BBO500S1-BBF/ H/ D-5.0-Z SP)

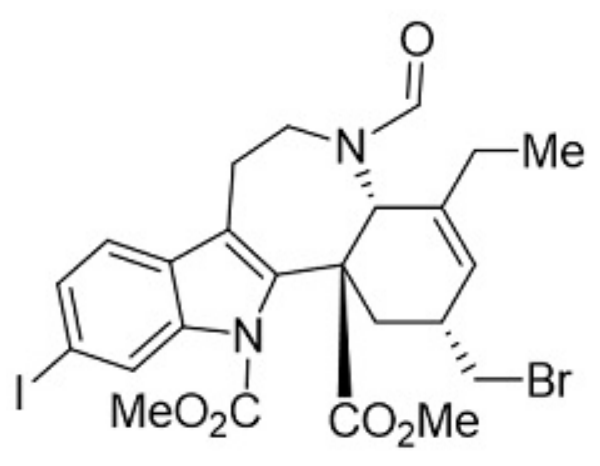

24

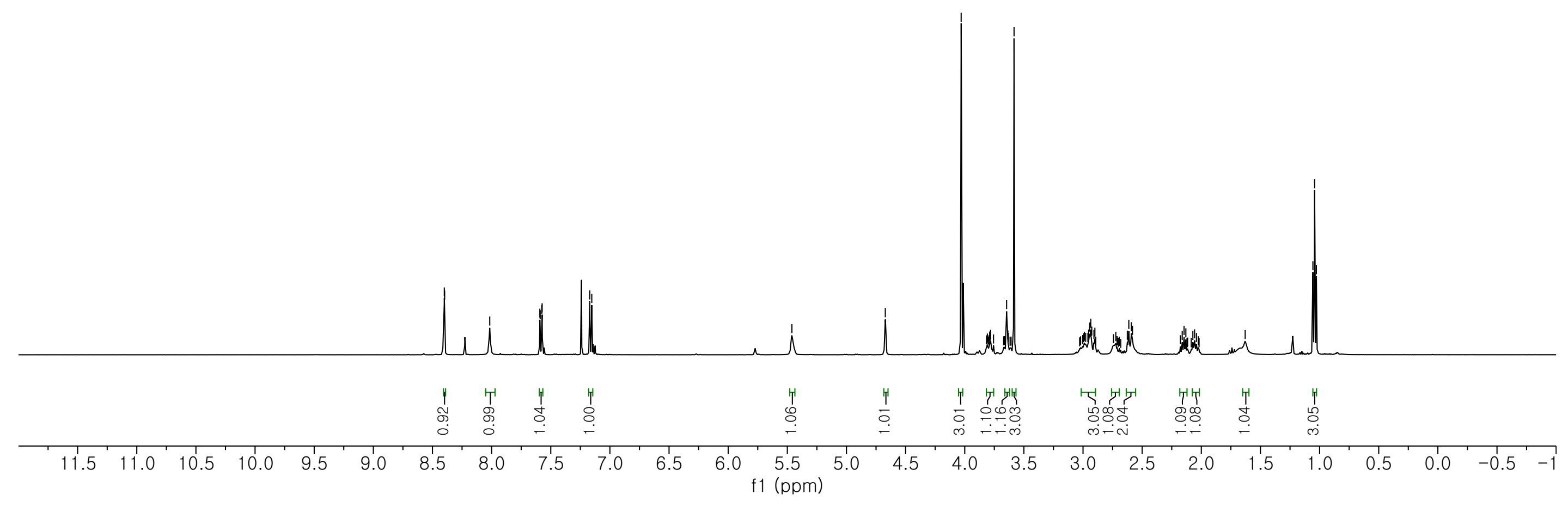


Bio-Patterned Reorganization of Alkaloids Enabled by Ring-Opening Functionalization of Tertiary Amines

Lim, H.; Seong, S.; Kim, Y.; Seo, S.; Han, S.*

|| $\quad$ |

\section{Parameter}

Value

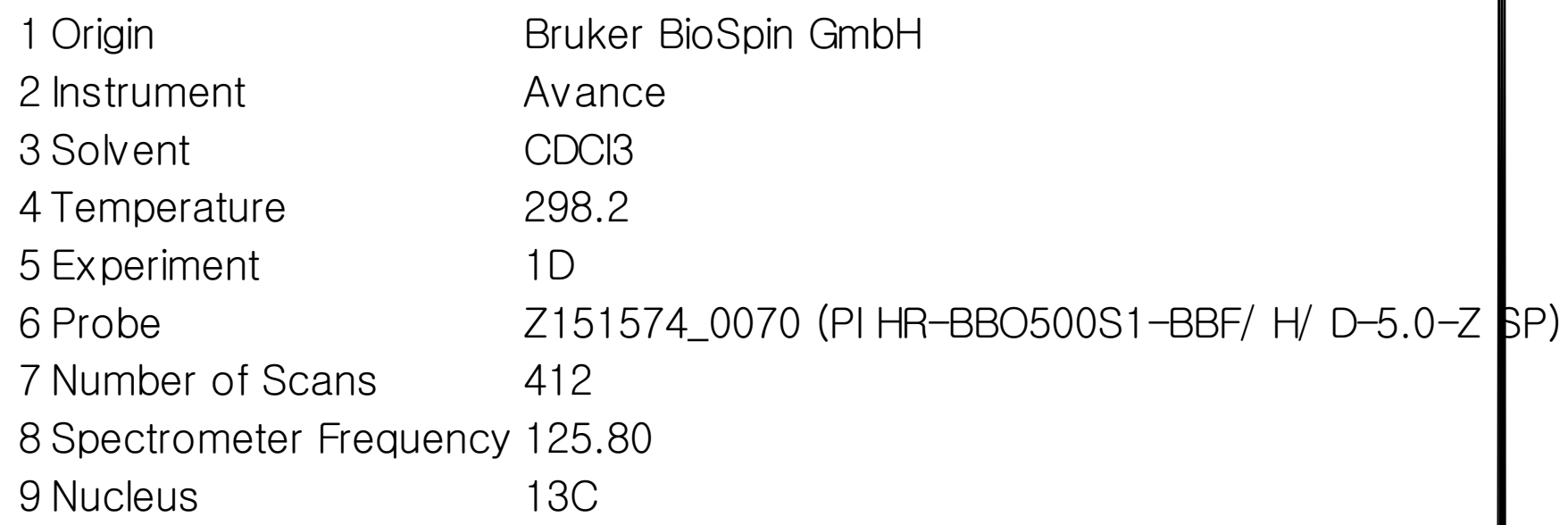

9 Nucleus

$13 \mathrm{C}$

Bruker BioSpin $\mathrm{GmbH}$

Avance

$\mathrm{CDCl} 3$

298.2

10

Z151574_0070 (PI HR-BBO500S1-BBF/ H/ D-5.0-Z SP)

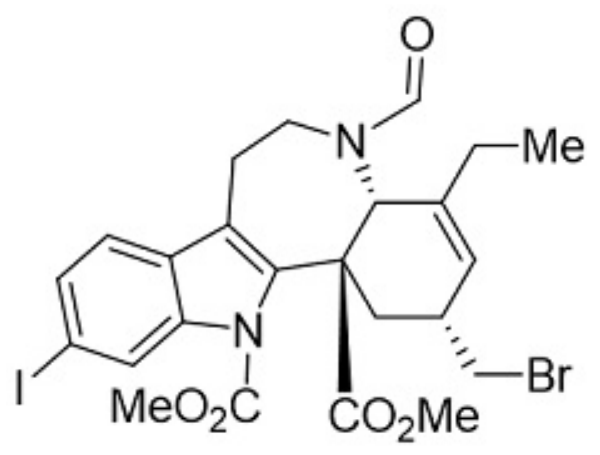

24

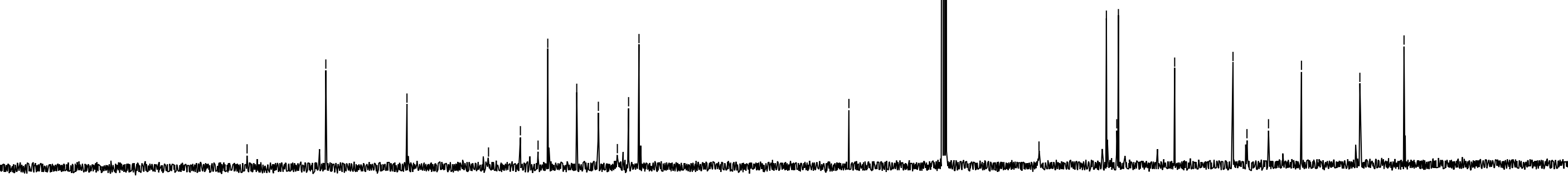




\section{Parameter}

Value

1 Origin

2 Instrument

3 Solvent

4 Temperature

5 Experiment

6 Probe

7 Number of Scans

8 Spectrometer Frequency 500.

9 Nucleus
Bruker BioSpin $\mathrm{GmbH}$

Avance

$\mathrm{CDCl} 3$

298.0

10

Z151574_0070 (PI HR-BBO500S1-BBF/ H/ D-5.0-Z SP)

16

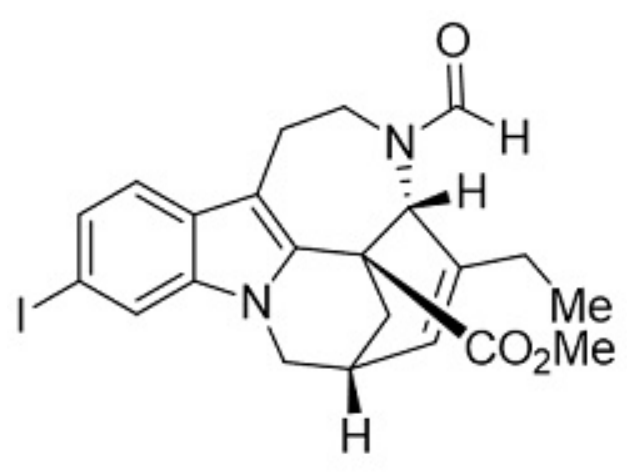

25

$1 \mathrm{H}$

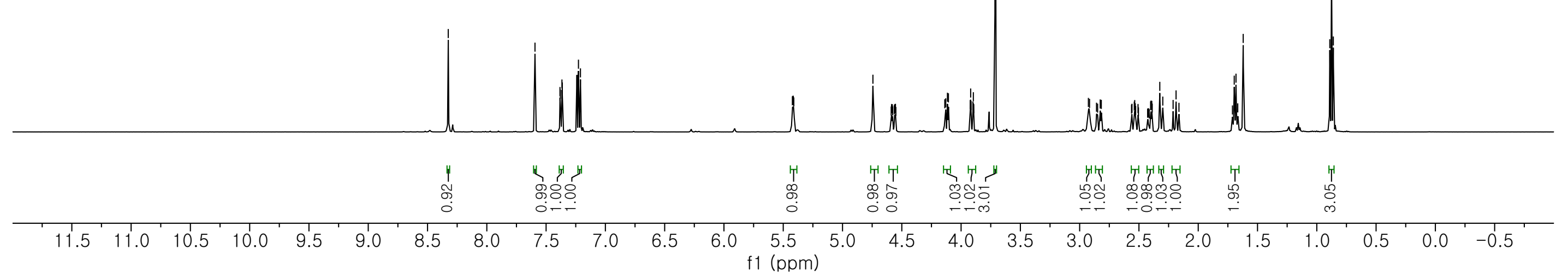




\section{Parameter}

1 Origin

2 Instrument

3 Solvent

4 Temperature

5 Experiment

6 Probe

7 Number of Scans

8 Spectrometer Frequency 12

9 Nucleus

10

\section{Value}

Bruker BioSpin GmbH

Avance

$\mathrm{CDCl} 3$

298.2

Z151574_0070 (PI HR-BBO500S1-BBF/ H/ D-5.0-Z PP) 104

125.80

$13 \mathrm{C}$

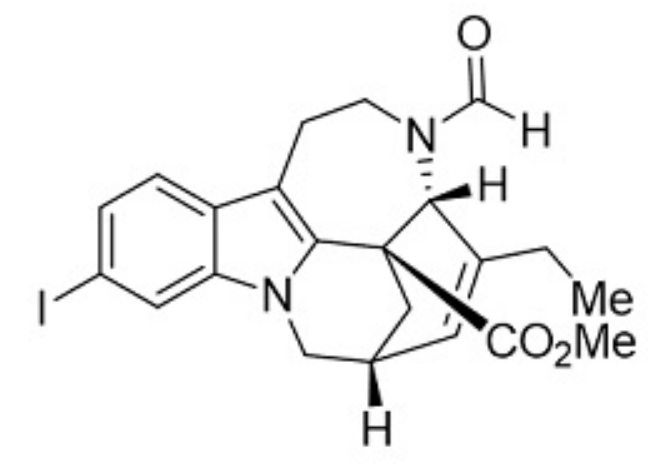

25 


\section{Parameter}

1 Origin

2 Instrument

3 Solvent

4 Temperature

5 Experiment

6 Probe

7 Number of Scans

8 Spectrometer Frequency 500.23

9 Nucleus

CDCl3

298.0

10

14

$1 \mathrm{H}$
Value

Bruker BioSpin GmbH

Avance

Z151574_0070 (PI HR-BBO500S1-BBF/ H/ D-5.0-Z SP)

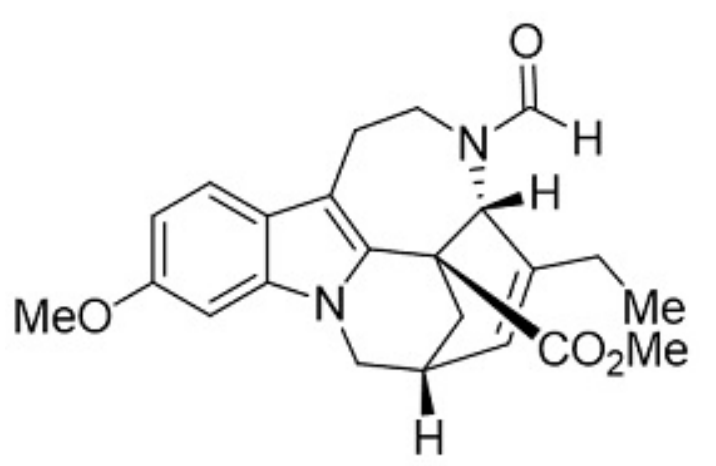

S4

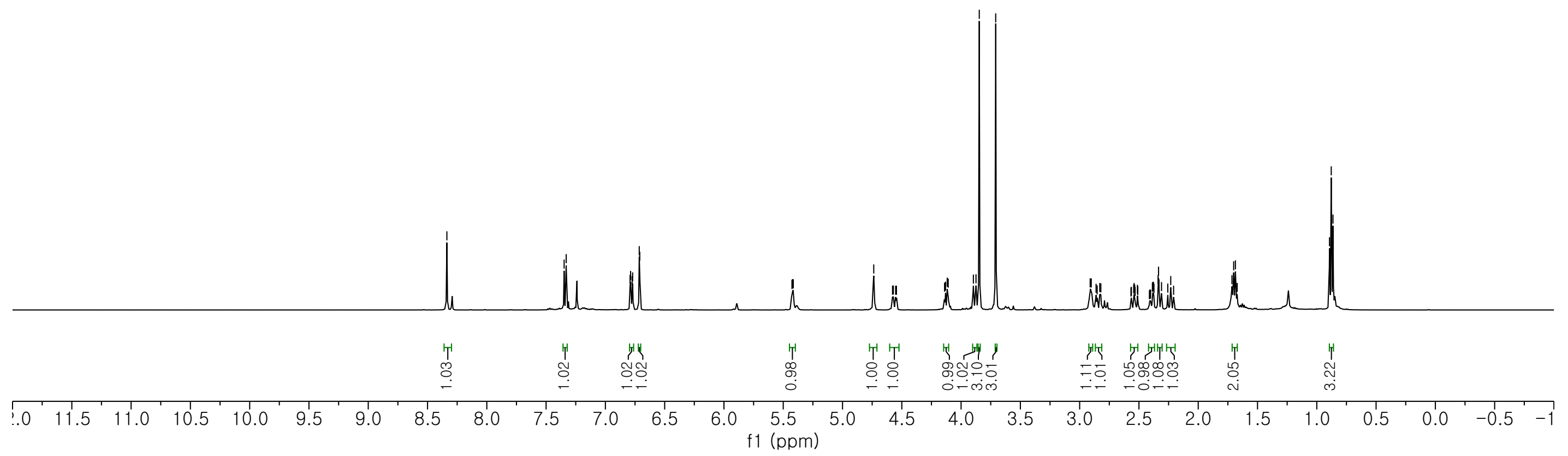




\section{Parameter}

1 Origin

2 Instrument

3 Solvent

4 Temperature

5 Experiment

6 Probe

7 Number of Scans

8 Spectrometer Frequency 125

9 Nucleus
Value

Bruker BioSpin GmbH

Avance

$\mathrm{CDCl} 3$

298.2

10

Z151574_0070 (PI HR-BBO500S1-BBF/ H/ D-5.0-Z SP)

64

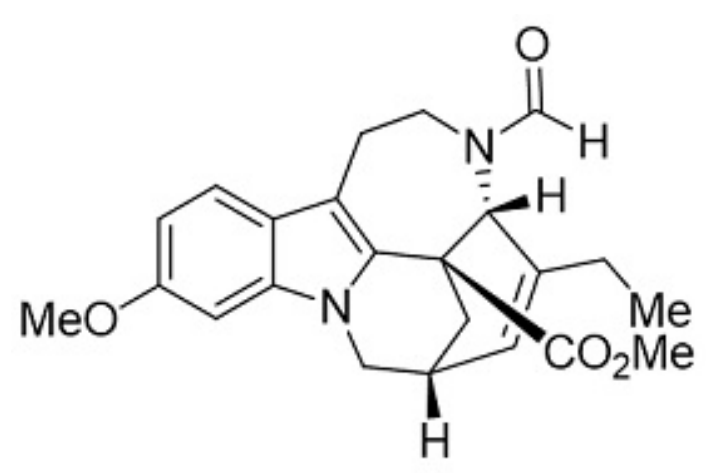

S4 


\section{Parameter}

\section{Value}

1 Origin

2 Instrument

3 Solvent

4 Temperature

5 Experiment

6 Probe

7 Number of Scans

8 Spectrometer Frequency 500.23

9 Nucleus

$\mathrm{CDCl} 3$

298.0

10

24

$1 \mathrm{H}$
Bruker BioSpin GmbH

Avance

Z151574_0070 (PI HR-BBO500S1-BBF/ H/ D-5.0-Z SP)

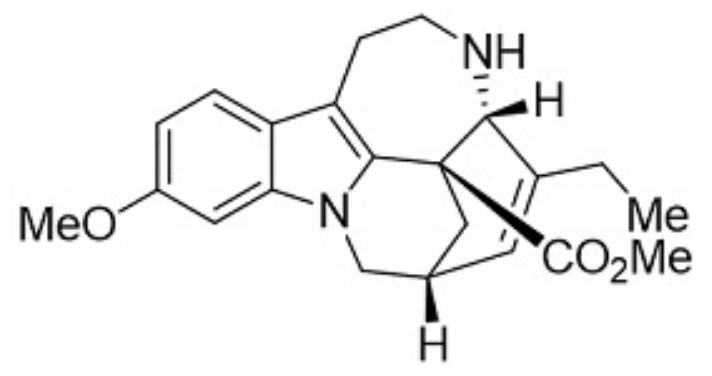

26 


\section{Parameter}

Value

$\begin{array}{ll}\text { 1 Origin } & \text { Varian } \\ 2 \text { Instrument } & \text { vnmrs } \\ \text { 3 Solvent } & \text { cdcl3 } \\ \text { 4 Temperature } & 25.0 \\ 5 \text { Experiment } & 10 \\ 6 \text { Probe } & 5 \mathrm{~mm} \_O n e \_p r o b e \\ 7 \text { Number of Scans } & 10772\end{array}$

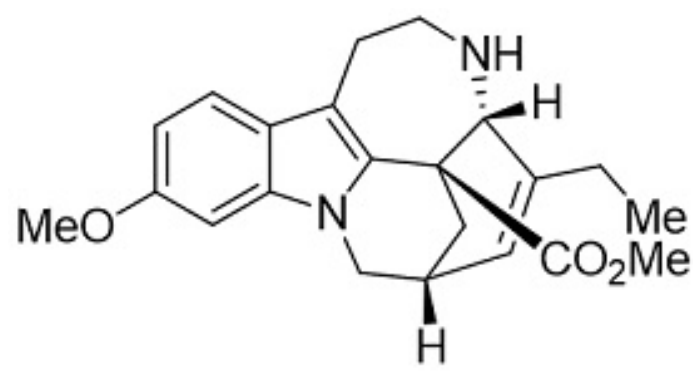

26 8 Spectrometer Frequency 150.86

9 Nucleus $13 \mathrm{C}$ 


\section{Parameter}

1 Origin

2 Instrument

3 Solvent

4 Temperature

5 Experiment

6 Probe

7 Number of Scans

8 Spectrometer Frequency 500.23

9 Nucleus

CDCl3

298.0

10

13

$1 \mathrm{H}$

\section{Value}

Bruker BioSpin GmbH

Avance

Z151574_0070 (PI HR-BBO500S1-BBF/ H/ D-5.0-Z SP)

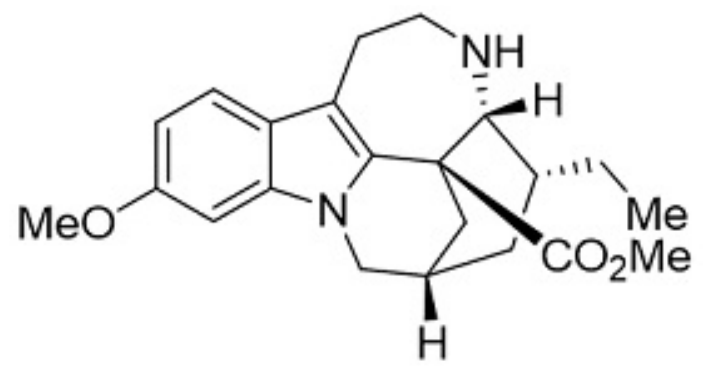

27

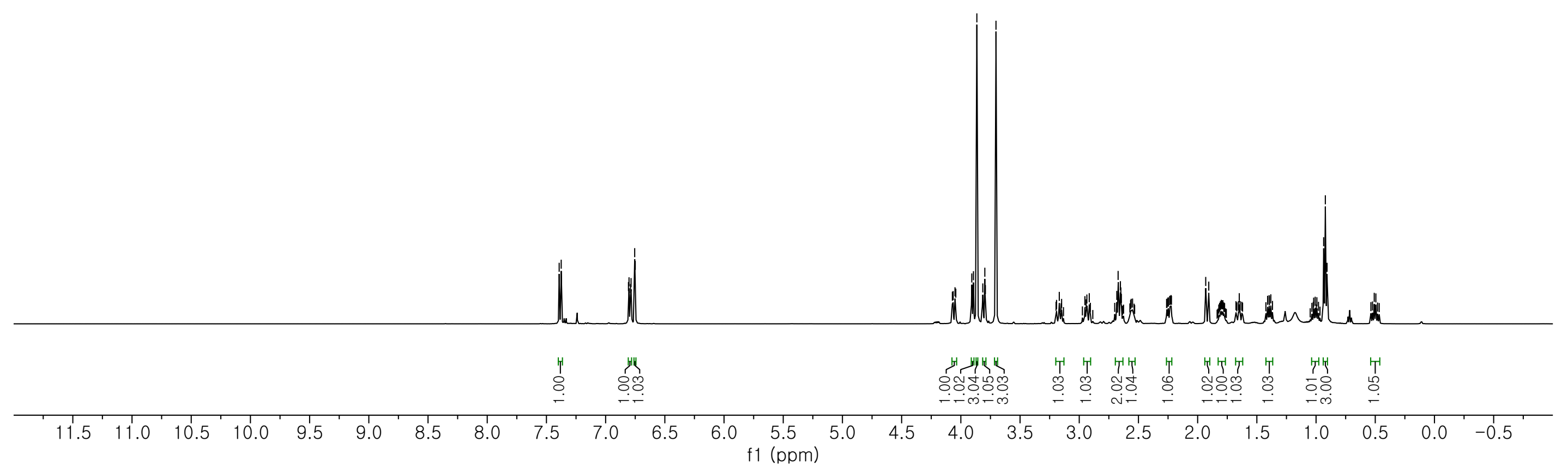




\section{Parameter}

\section{Value}

\section{Origin}

Varian

2 Instrument

vnmrs

3 Solvent

$\mathrm{cdcl} 3$

4 Temperature

25.0

5 Experiment

10

6 Probe

$5 \mathrm{~mm} \_$One_probe

7 Number of Scans

50

8 Spectrometer Frequency 150.86

9 Nucleus

$13 \mathrm{C}$

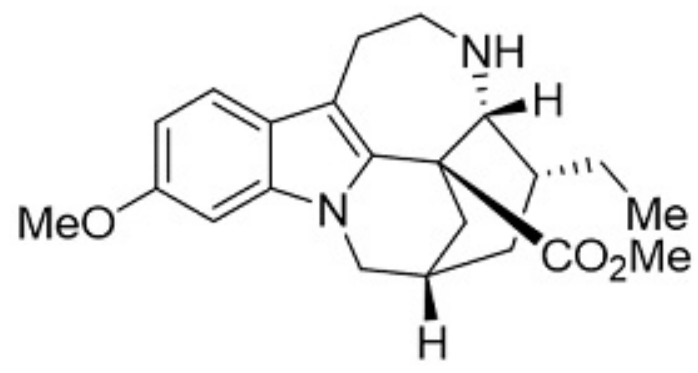

27

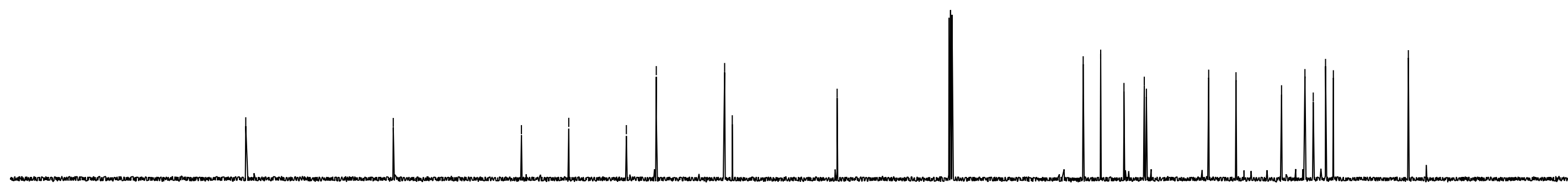




\section{Parameter}

\section{Origin}

2 Instrument

3 Solvent

4 Temperature

5 Experiment

6 Probe

7 Number of Scans

8 Spectrometer Frequency 500.23

9 Nucleus

$\mathrm{CDCl} 3$

298.0

10

11

$1 \mathrm{H}$

\section{Value}

Bruker BioSpin GmbH

Avance

Z151574_0070 (PI HR-BBO500S1-BBF/ H/ D-5.0-Z SP)

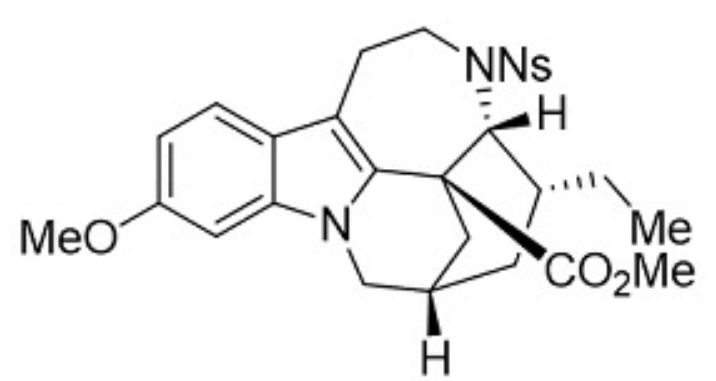

46

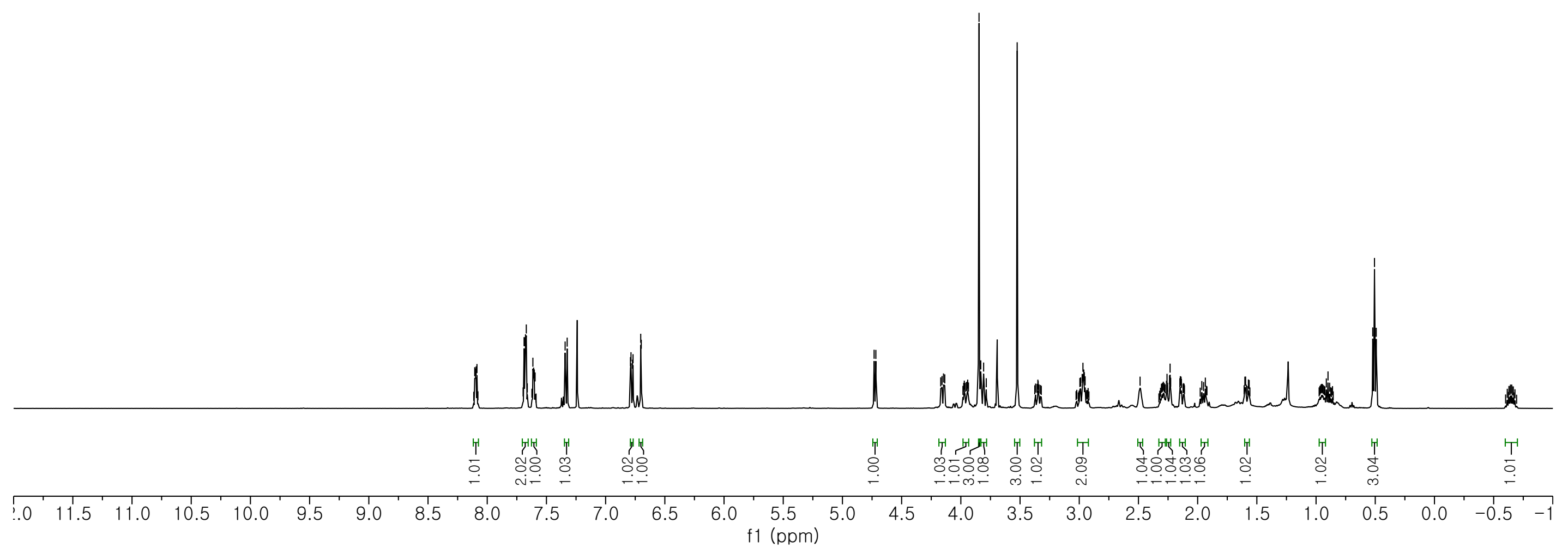




\section{Parameter}

Value

\section{Origin}

2 Instrument

Bruker BioSpin GmbH

3 Solvent

Avance

$\mathrm{CDCl} 3$

4 Temperature

298.2

5 Experiment

10

6 Probe

Z151574_0070 (PI HR-BBO500S1-BBF/ H/ D-5.0-Z SP)

7 Number of Scans 606

8 Spectrometer Frequency 125.80

9 Nucleus

$13 \mathrm{C}$

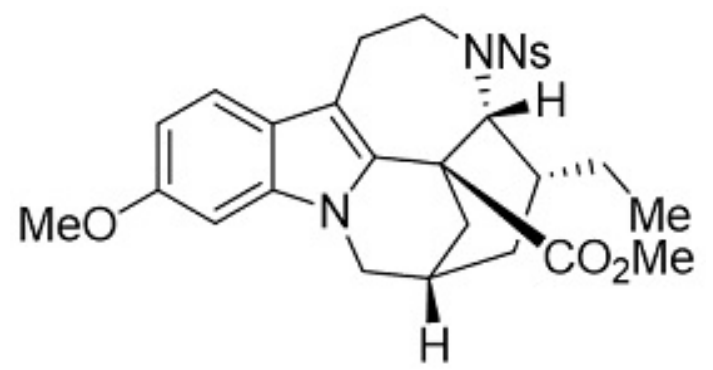

46 


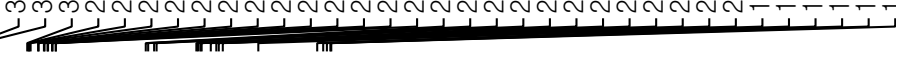

Parameter

Value

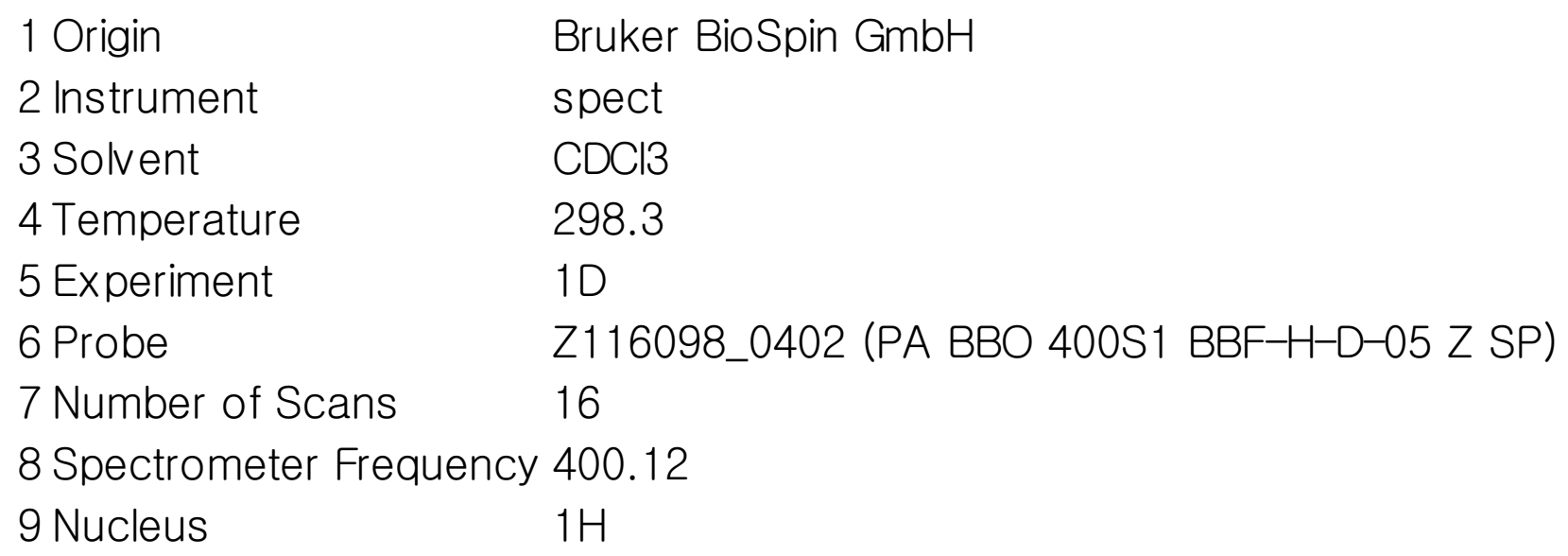

16

6 Probe

7 Number of Scans

$1 \mathrm{H}$

9 Nucleus
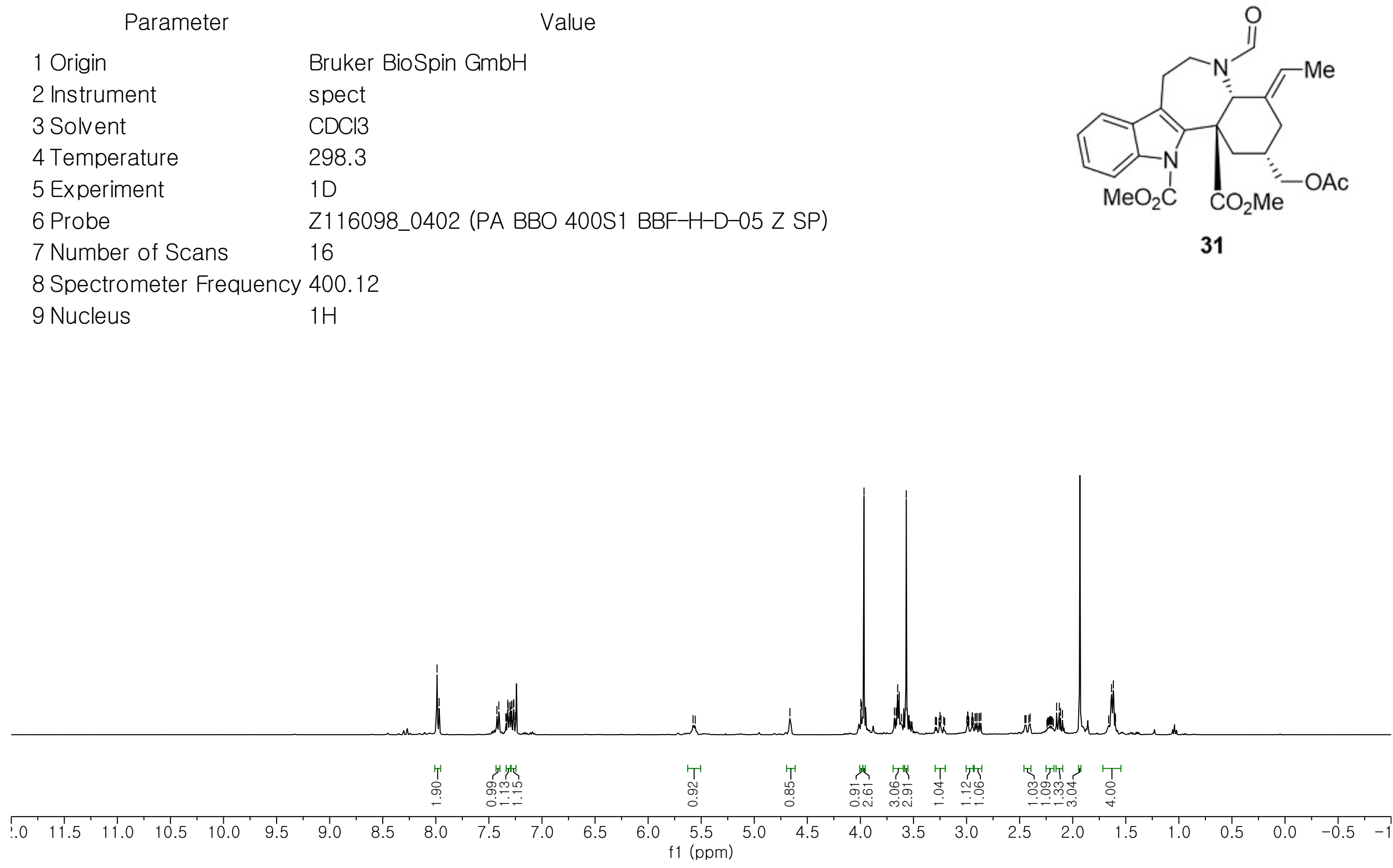


\section{Parameter}

1 Origin

2 Instrument

3 Solvent

4 Temperature

5 Experiment

6 Probe

7 Number of Scans

8 Spectrometer Fre

9 Nucleus

\section{Value}

\section{Bruker BioSpin $\mathrm{GmbH}$}

spect

$\mathrm{CDCl} 3$

298.3

$1 D$

Z116098_0402 (PA BBO 400S1 BBF-H-D-05 Z SP) 1200

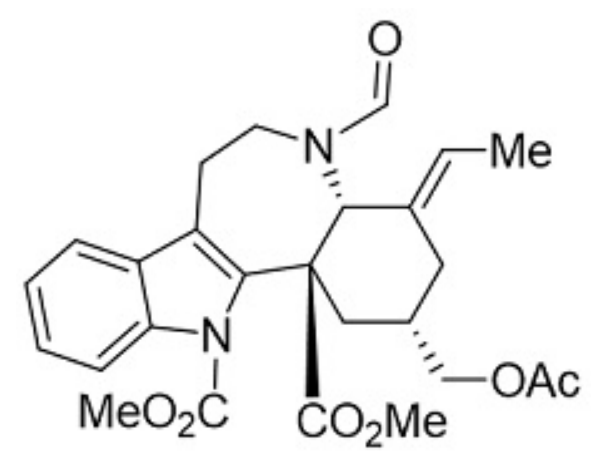

31

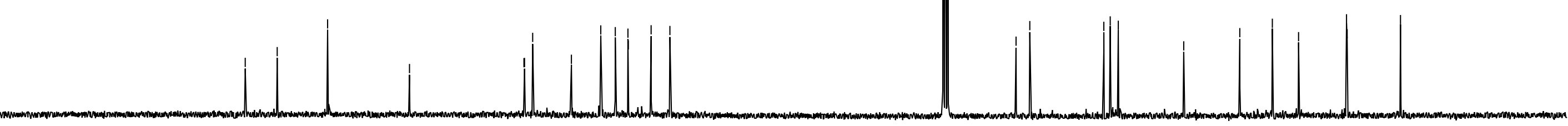




\section{Value}

1 Origin

2 Instrument

3 Solvent

4 Temperature

5 Experiment

6 Probe

7 Number of Scans

8 Spectrometer Frequency 400.13

9 Nucleus
UXNMR, Bruker Analytische Messtechnik GmbH

spect

$\mathrm{CDCl} 3$

293.2

10

5 mm Dual 13C/ 1H Z8246/ 0025

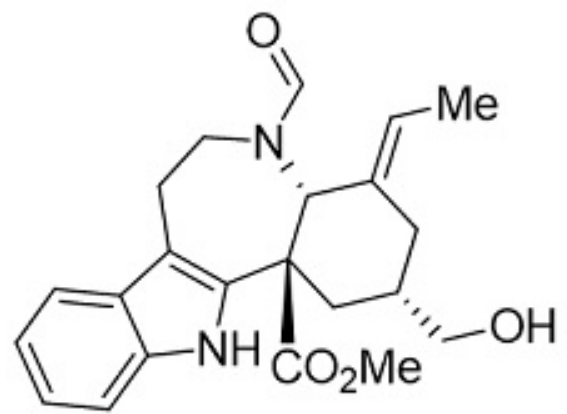

S5

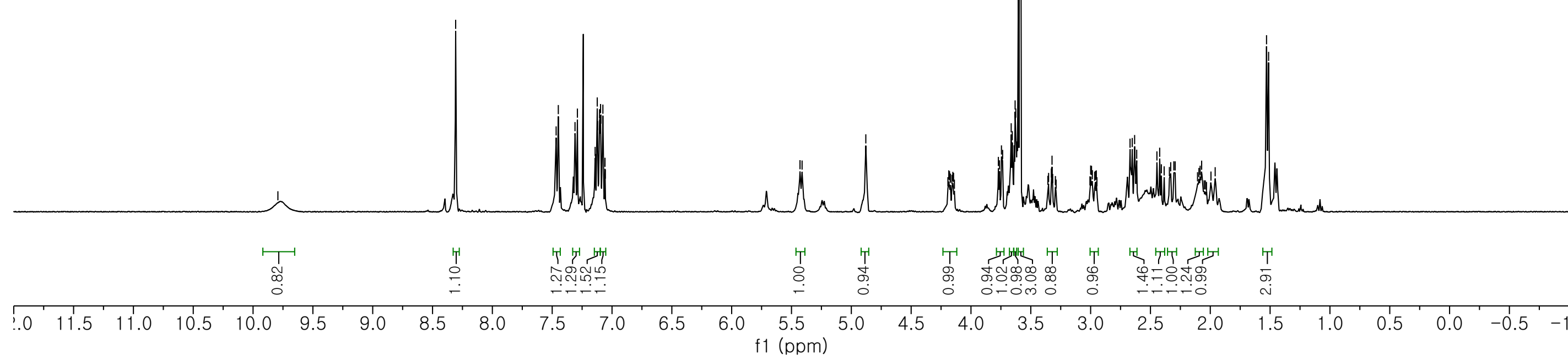




\section{Parameter}

1 Origin

2 Instrument

3 Solvent

4 Temperature

5 Experiment

6 Probe

7 Number of Scans

Value

Bruker BioSpin GmbH

spect

$\mathrm{CDCl} 3$

298.3

10

Z116098_0402 (PA BBO 400S1 BBF-H-D-05 Z SP) 696

8 Spectrometer Frequency 100.62

9 Nucleus

$13 \mathrm{C}$

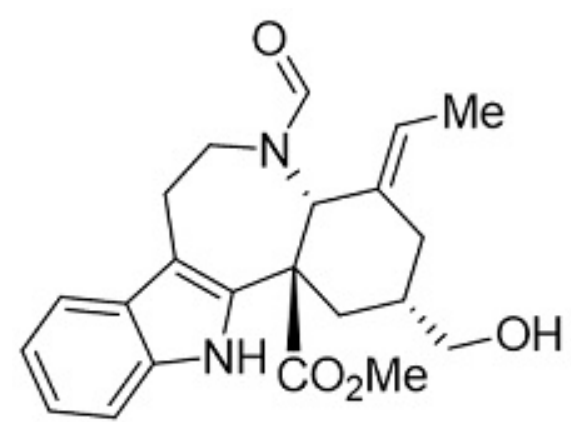

S5 


\section{Parameter}

1 Origin

2 Instrument

3 Solvent

4 Temperature

5 Experiment

6 Probe

7 Number of Scans

8 Spectrometer Frequency 400.12

9 Nucleus

spect

$\mathrm{CDCl} 3$

298.0

10

16

$1 \mathrm{H}$

\section{Value}

Bruker BioSpin $\mathrm{GmbH}$

Z116098_0402 (PA BBO 400S1 BBF-H-D-05 Z SP)

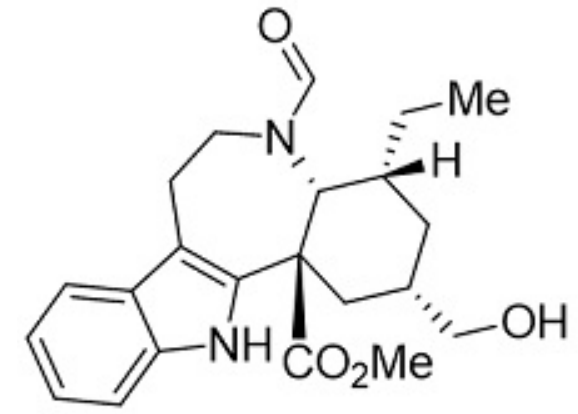

32

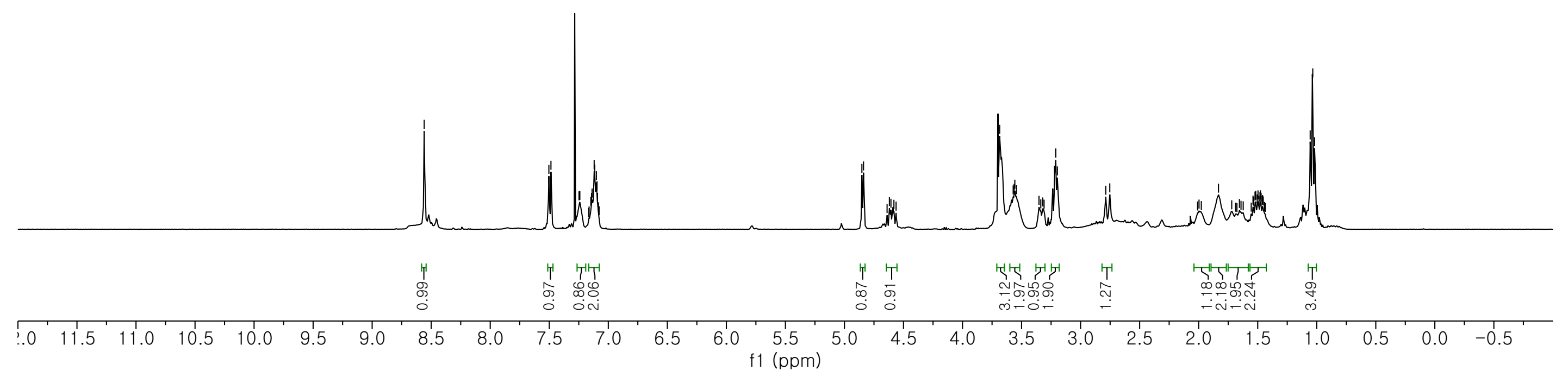




\section{Parameter}

1 Origin

2 Instrument

3 Solvent

4 Temperature

5 Experiment

6 Probe

7 Number of Scans

8 Spectrometer Frequency 125.80

9 Nucleus

$13 \mathrm{C}$

CDCl3

298.2

10 456

\section{Value}

Bruker BioSpin GmbH

Avance

Z151574_0070 (PI HR-BBO500S1-BBF/ H/ D-5.0-Z PP)

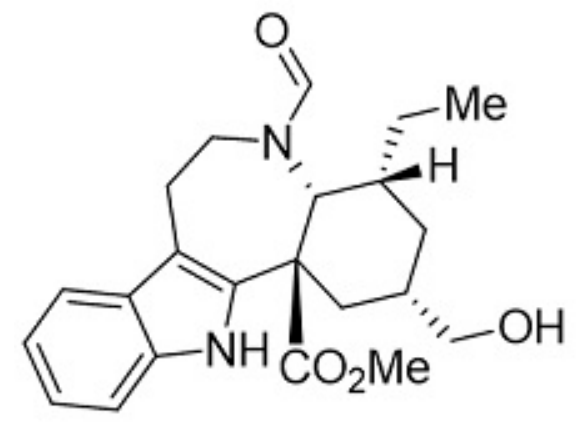

32

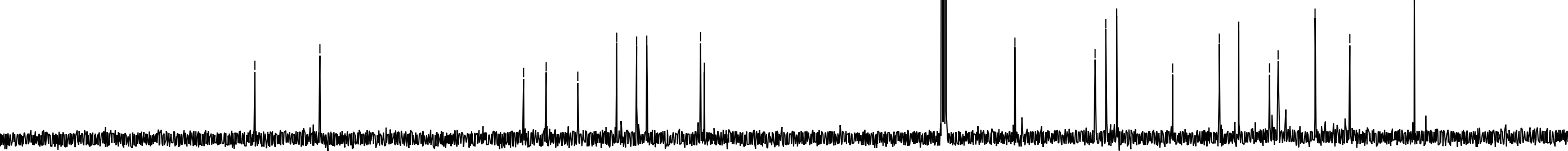




\section{Parameter}

1 Origin

2 Instrument

3 Solvent

4 Temperature

5 Experiment

6 Probe

7 Number of Scans

8 Spectrometer Frequency 400.13

9 Nucleus

spect

CDCl3

298.0

$1 \mathrm{D}$

30

$1 \mathrm{H}$

H
Value

Bruker BioSpin GmbH

Z108618_0389 (PA BBO 400S1 BBF-H-D-05 Z PLUS)

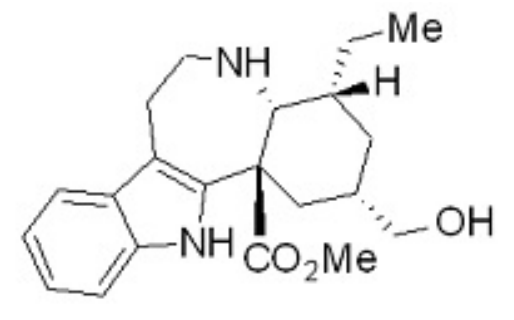

(+)-3-hydroxy-3,4-secocoronaridine (33) 
Parameter

\section{Origin}

2 Instrument

3 Solvent

4 Temperature

5 Experiment

6 Probe

7 Number of Scans

$\begin{array}{ll}8 \text { Spectrometer Frequency } 100.62 \\ 9 \text { Nucleus } & 13 \mathrm{C}\end{array}$

spect

$\mathrm{CDCl} 3$

298.0

$1 \mathrm{D}$ 4000

\section{Value}

Bruker BioSpin $\mathrm{GmbH}$

Z116098_0402 (PA BBO 400S1 BBF-H-D-05 Z SP)

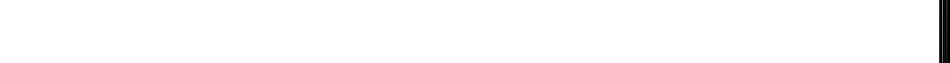

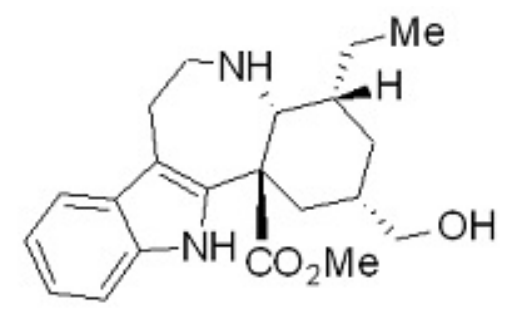

(+)-3-hydroxy-3,4-secocoronaridine (33) 


\section{Parameter}

1 Origin

2 Instrument

3 Solvent

4 Temperature

5 Experiment

6 Probe

7 Number of Scans

8 Spectrometer Frequency 400.12

9 Nucleus

spect

$\mathrm{CDCl} 3$

298.0

$1 \mathrm{D}$

32

$1 \mathrm{H}$

\section{Value}

Bruker BioSpin GmbH

Z116098_0402 (PA BBO 400S1 BBF-H-D-05 Z SP)

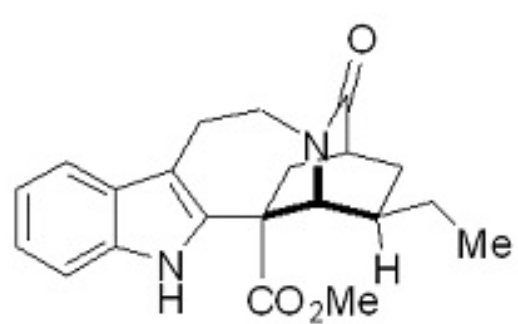

(+)-3-oxocoronaridine (35)

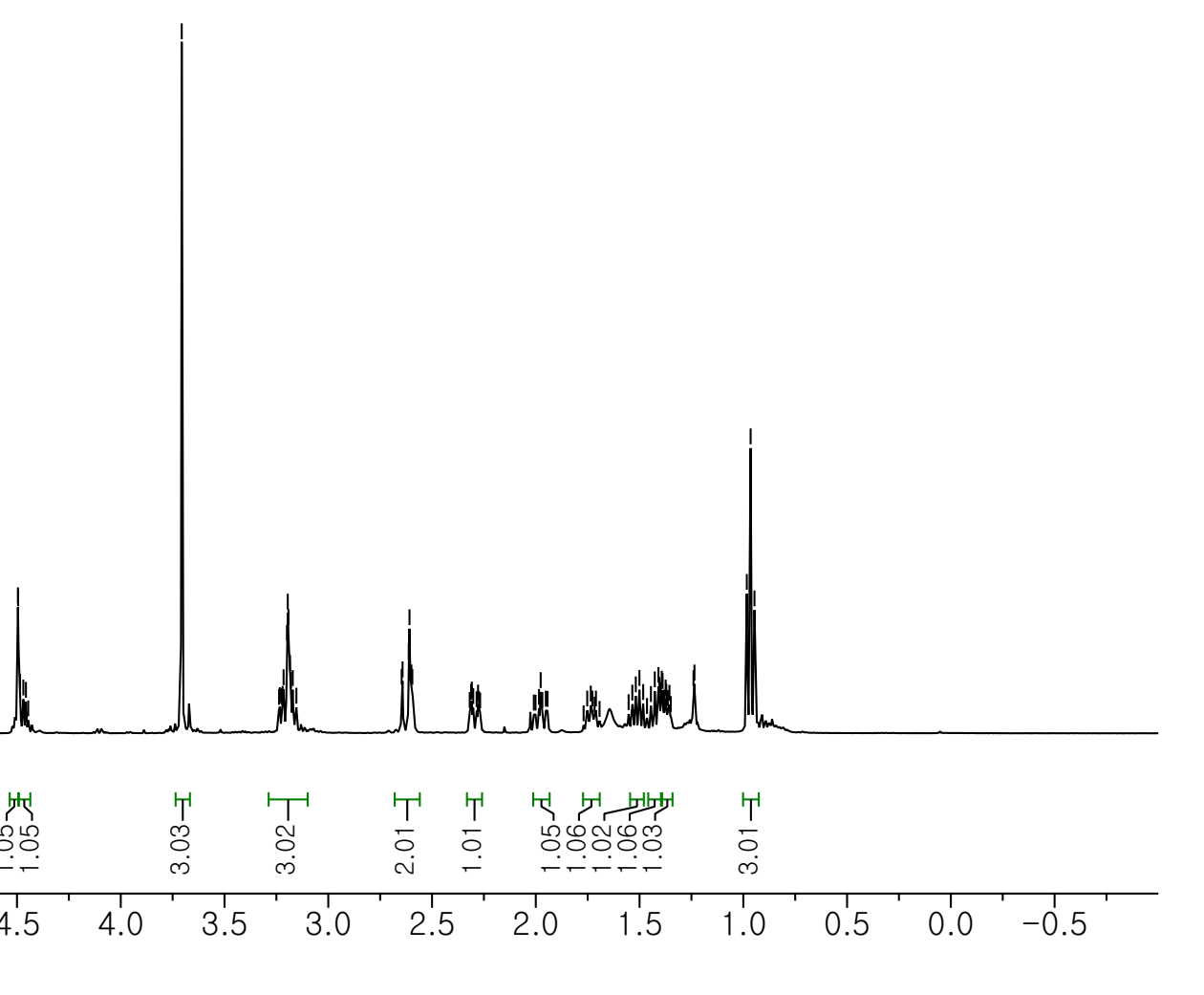




\section{Parameter}

Value

\section{Origin}

2 Instrument

3 Solvent

4 Temperature

5 Experiment

6 Probe

7 Number of Scans

8 Spectrometer Frequency 100.62

9 Nucleus

spect

$\mathrm{CDCl} 3$

298.0

$1 \mathrm{D}$ 4000

$13 \mathrm{C}$
Bruker BioSpin GmbH

Z116098_0402 (PA BBO 400S1 BBF-H-D-05 Z Sf)

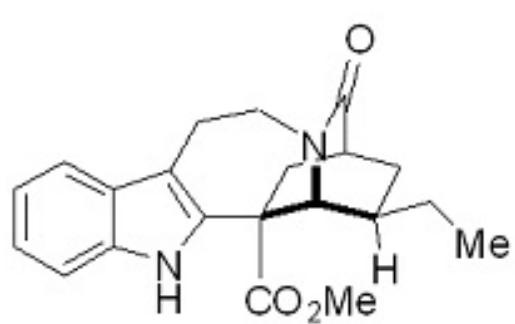

(+)-3-oxocoronaridine (35)

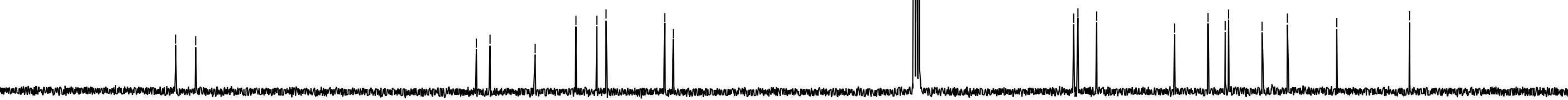




\section{Parameter}

1 Origin

2 Instrument

3 Solvent

4 Temperature

5 Experiment

6 Probe

7 Number of Scans

8 Spectrometer Frequency 400.12

9 Nucleus

spect

$\mathrm{CDCl} 3$

298.0

10 20

$1 \mathrm{H}$
Bruker BioSpin GmbH

Z116098_0402 (PA BBO 400S1 BBF-H-D-05 Z SP)
Value

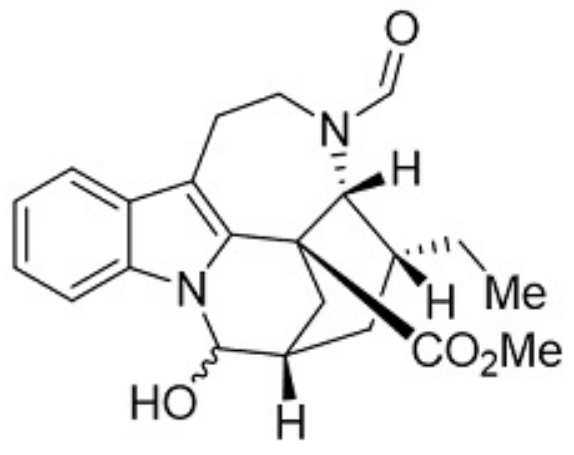

S6

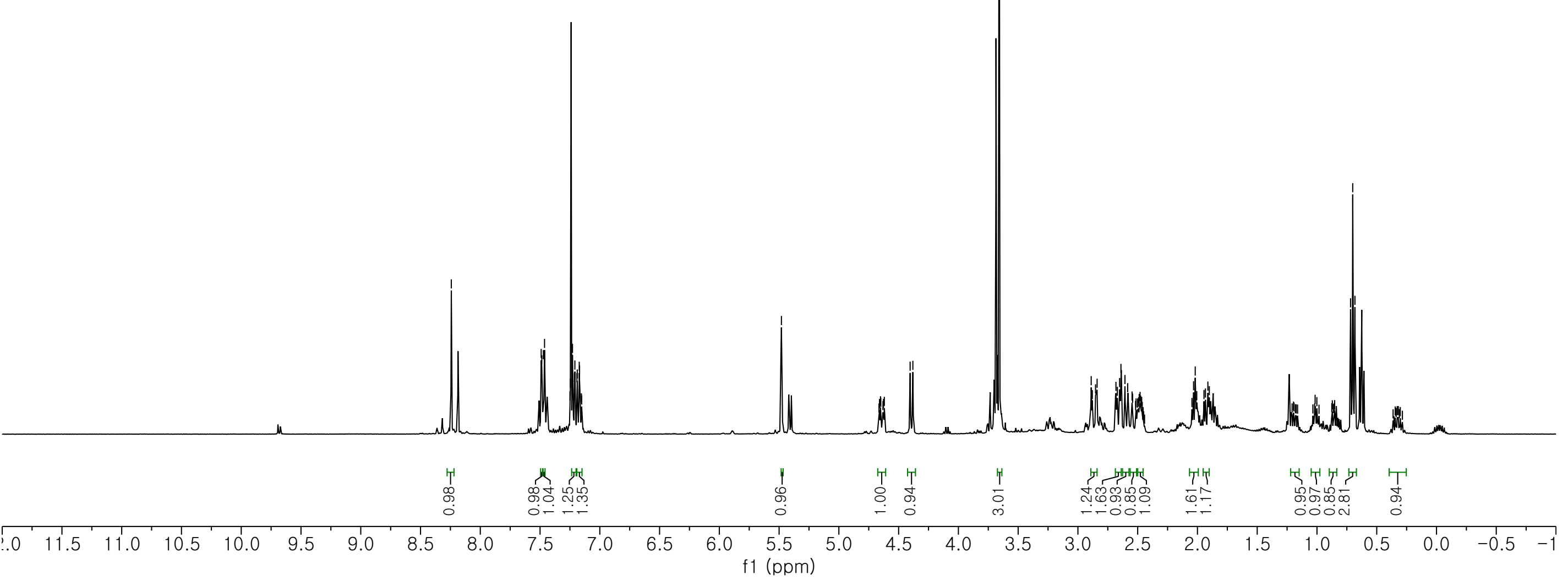




\section{Parameter}

1 Origin

2 Instrument

3 Solvent

4 Temperature

5 Experiment

6 Probe

7 Number of Scans

8 Spectrometer Frequency 100.62

9 Nucleus

$13 \mathrm{C}$

spect

$\mathrm{CDCl} 3$

298.0

$1 \mathrm{D}$ 696

\section{Value}

Bruker BioSpin $\mathrm{GmbH}$

Z116098_0402 (PA BBO 400S1 BBF-H-D-05 Z Sf)

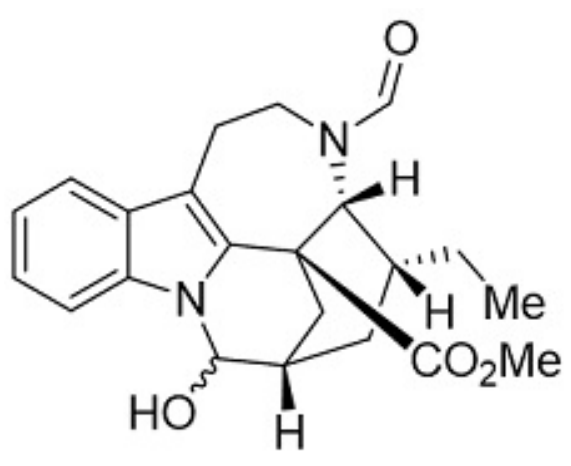

s6

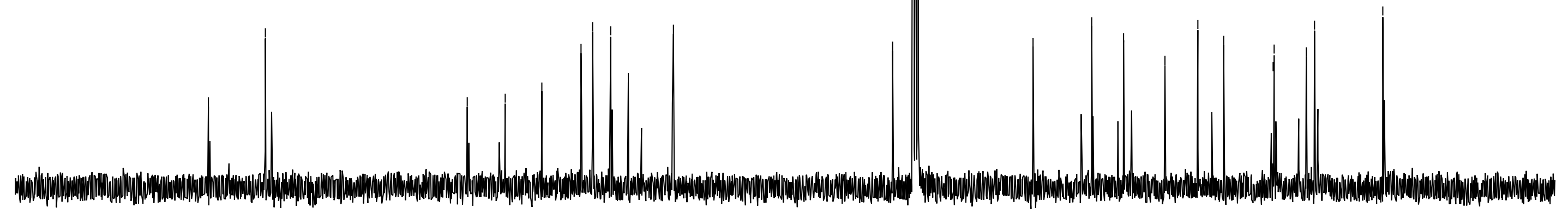




\section{Parameter}

1 Origin

2 Instrument

3 Solvent

4 Temperature

5 Experiment

6 Probe

7 Number of Scans

8 Spectrometer Frequency 400.12

9 Nucleus

spect

$\mathrm{CDCl} 3$

298.0

10 20

$1 \mathrm{H}$

\section{Value}

Bruker BioSpin GmbH

Z116098_0402 (PA BBO 400S1 BBF-H-D-05 Z SP)

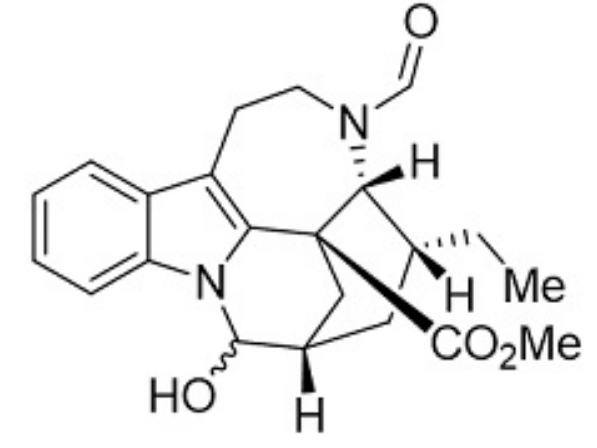

s6

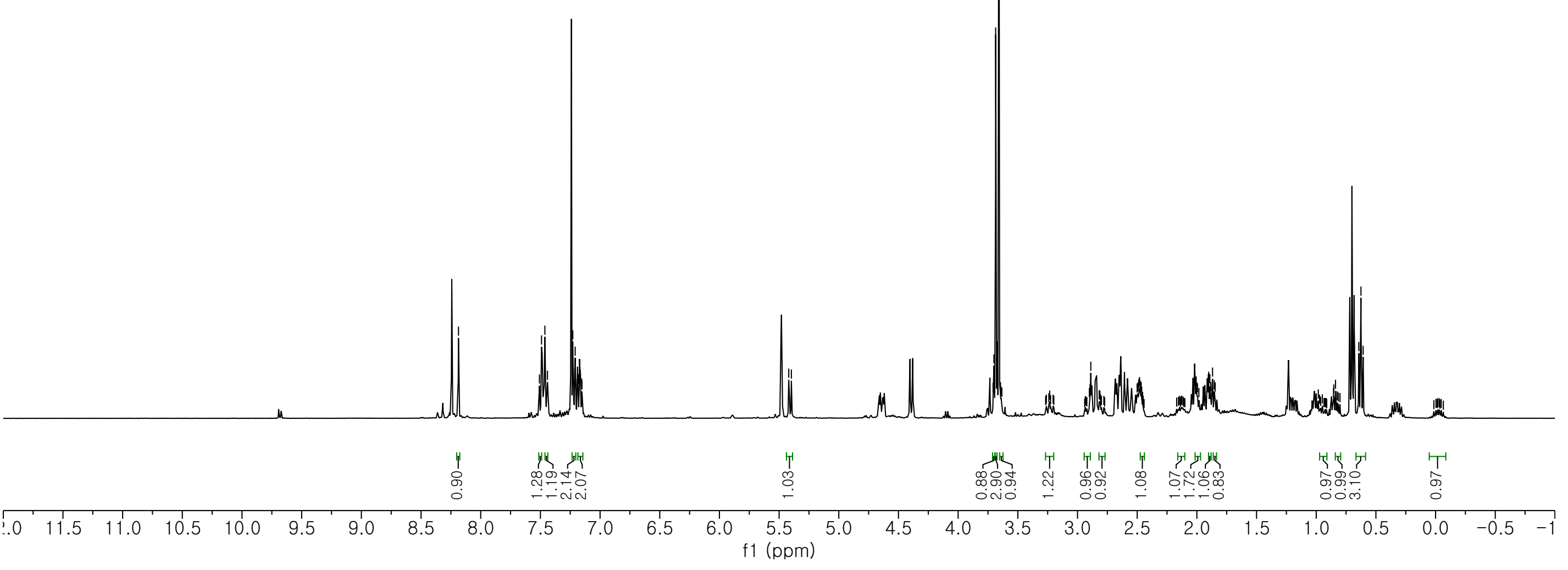




\section{Origin}

2 Instrument

3 Solvent

4 Temperature

5 Experiment

6 Probe

7 Number of Scans

8 Spectrometer Frequency 100.62

9 Nucleus

$13 \mathrm{C}$

spect

CDCl3

298.0

10 696
Bruker BioSpin GmbH

Z116098_0402 (PA BBO 400S1 BBF-H-D-05 Z SP)

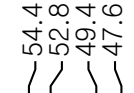

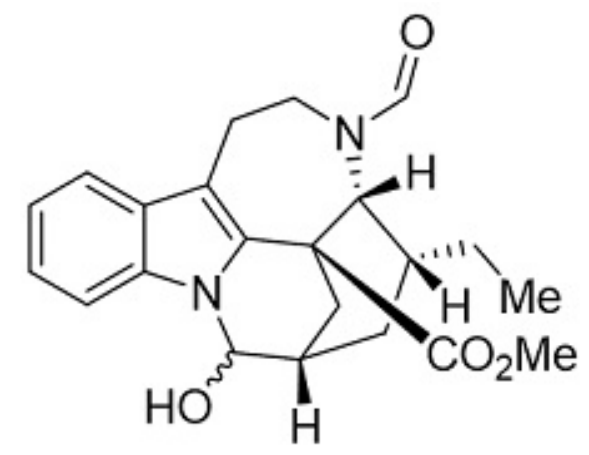

s6

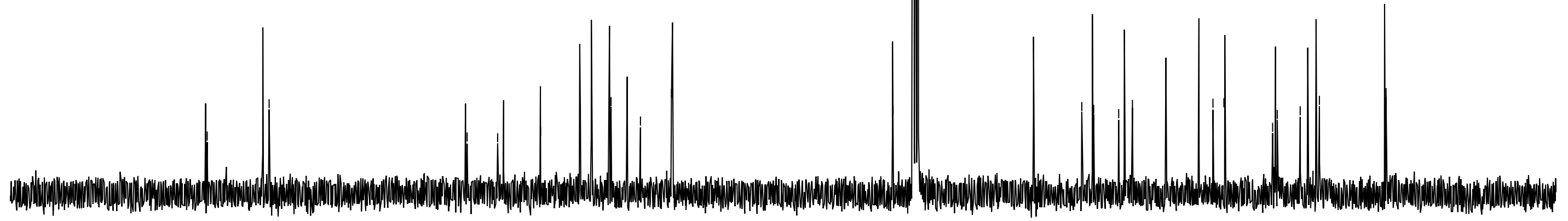




\section{Parameter}

1 Origin

2 Instrument

3 Solvent

4 Temperature

5 Experiment

6 Probe

7 Number of Scans

8 Spectrometer Frequency 500.23

9 Nucleus

$\mathrm{CDCl} 3$

298.0

10

9

$1 \mathrm{H}$

\section{Value}

Bruker BioSpin GmbH

Avance

Z151574_0070 (PI HR-BBO500S1-BBF/ H/ D-5.0-Z SP)

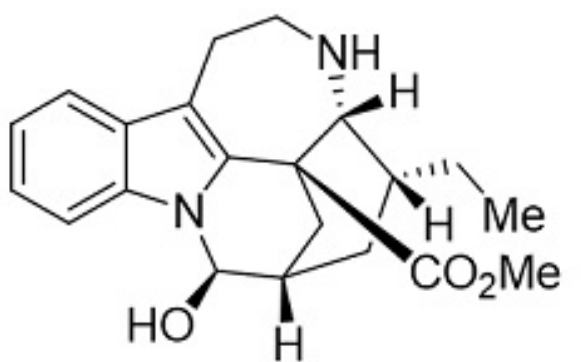

(+)-10,11-demethoxychippiine (4)

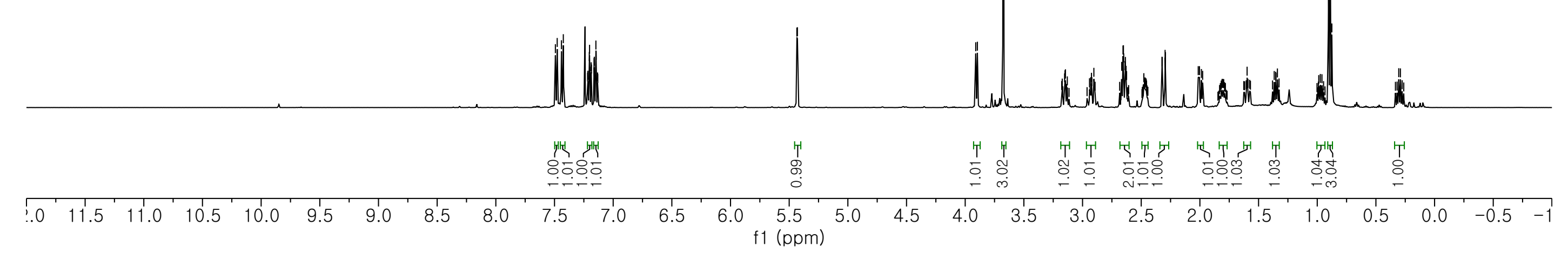




\section{Parameter}

Value

\section{Origin}

2 Instrument

3 Solvent

4 Temperature

5 Experiment

6 Probe

7 Number of Scans

8 Spectrometer Frequency 125.80

9 Nucleus

$\mathrm{CDCl} 3$

298.2

10

75

$13 \mathrm{C}$
Bruker BioSpin GmbH

Avance

Z151574_0070 (PI HR-BBO500S1-BBF/ H/ D-5.0-Z SP)

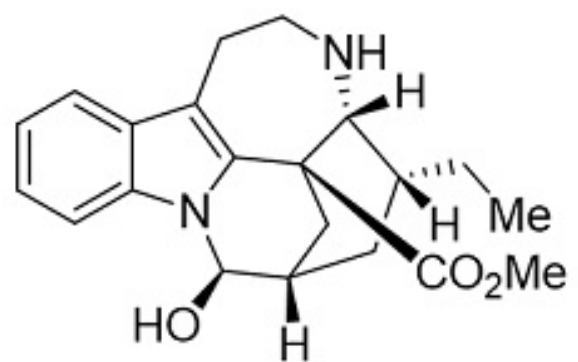

(+)-10,11-demethoxychippiine (4)

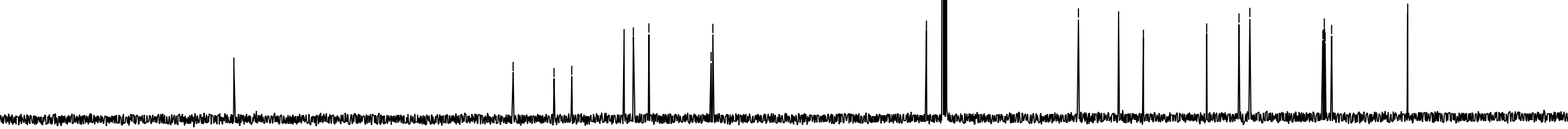




\section{Parameter}

\section{Value}

1 Origin

Varian

2 Instrument

vnmrs

3 Solvent

cdcl3

25.0

4 Temperature

10

5 Experiment

5mm_One_probe

6 Probe

16

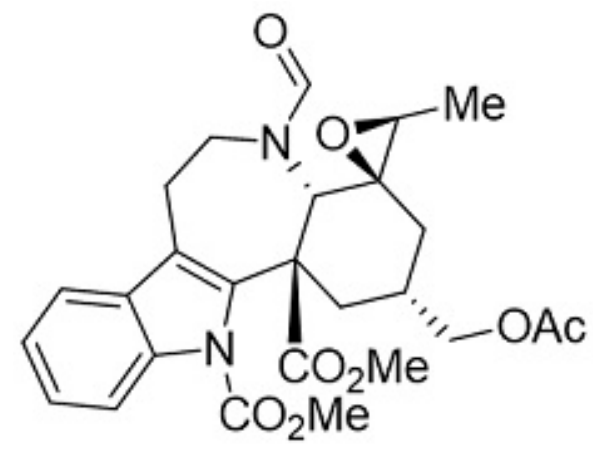

7 Number of Scans

S7

8 Spectrometer Frequency 599.93

9 Nucleus

$1 \mathrm{H}$

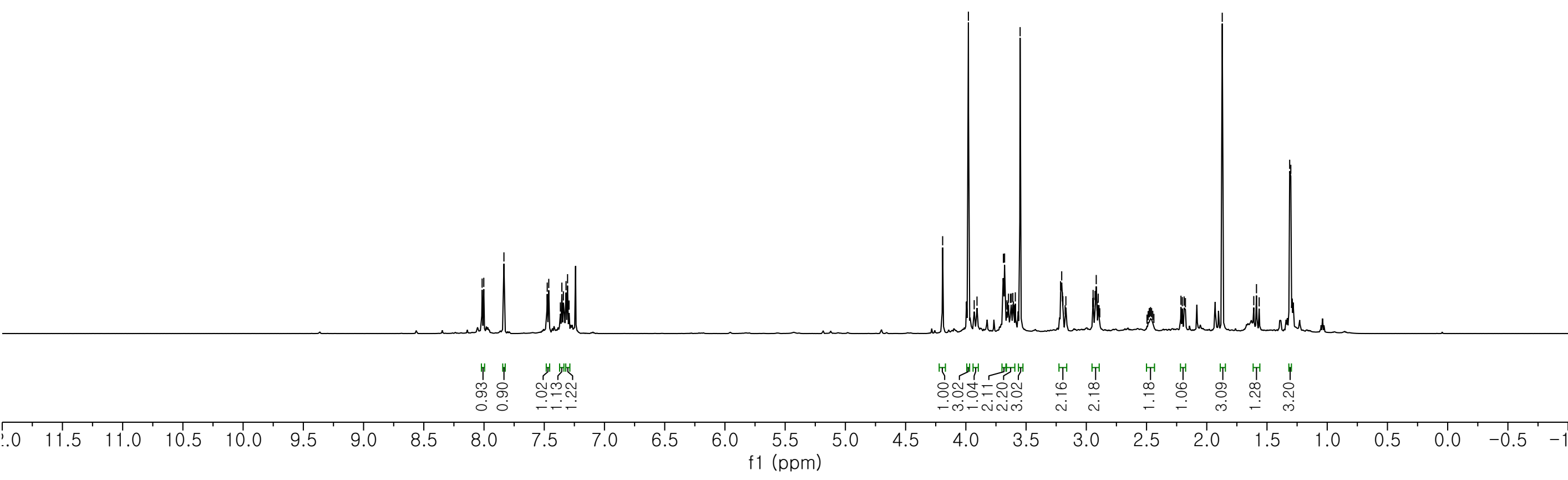




\begin{tabular}{ll}
\multicolumn{1}{c}{ Parameter } & \multicolumn{1}{c}{ Value } \\
1 Origin & Varian \\
2 Instrument & vnmrs \\
3 Solvent & cdcl3 \\
4 Temperature & 25.0 \\
5 Experiment & 10 \\
6 Probe & $5 \mathrm{~mm}$ OOne_probe \\
7 Number of Scans & 1200 \\
8 Spectrometer Frequency 150.87 \\
9 Nucleus & $13 \mathrm{C}$
\end{tabular}

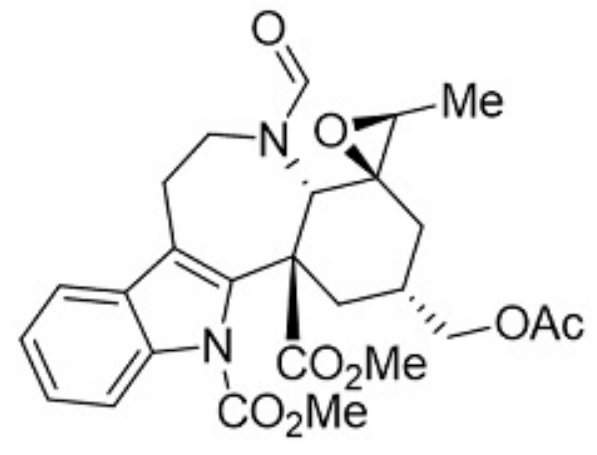

s7

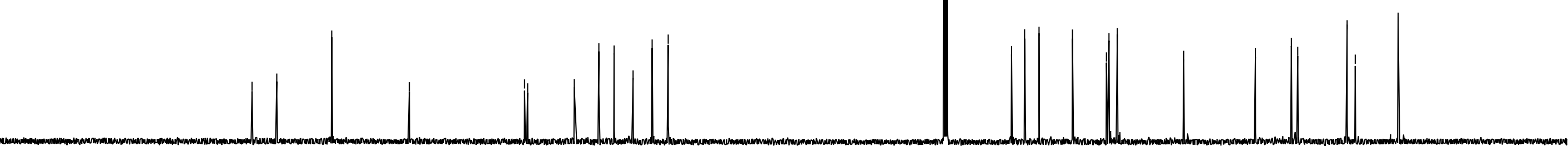


Parameter

\section{Origin}

2 Instrument

3 Solvent

4 Temperature

5 Experiment

6 Probe

7 Number of Scans

8 Spectrometer Frequency 400.12

9 Nucleus

spect

$\mathrm{CDCl} 3$

298.0

10

16

$1 \mathrm{H}$

\section{Value}

Bruker BioSpin GmbH

Z116098_0402 (PA BBO 400S1 BBF-H-D-05 Z SP)

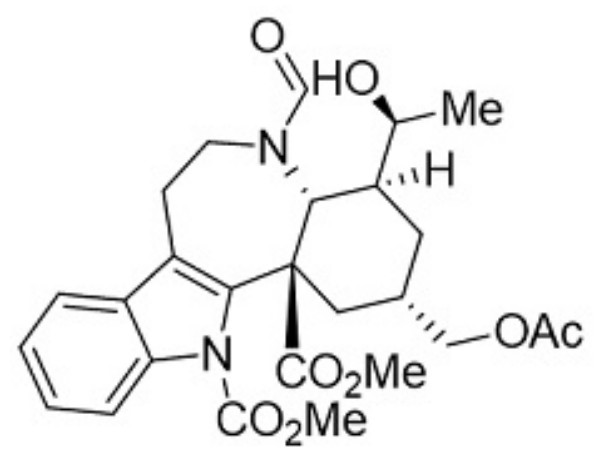

36

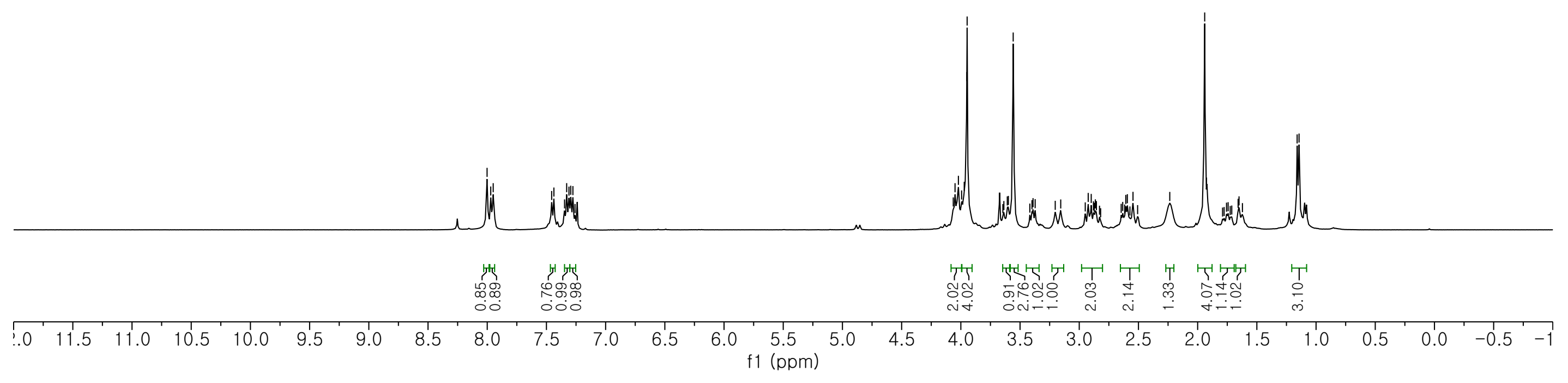



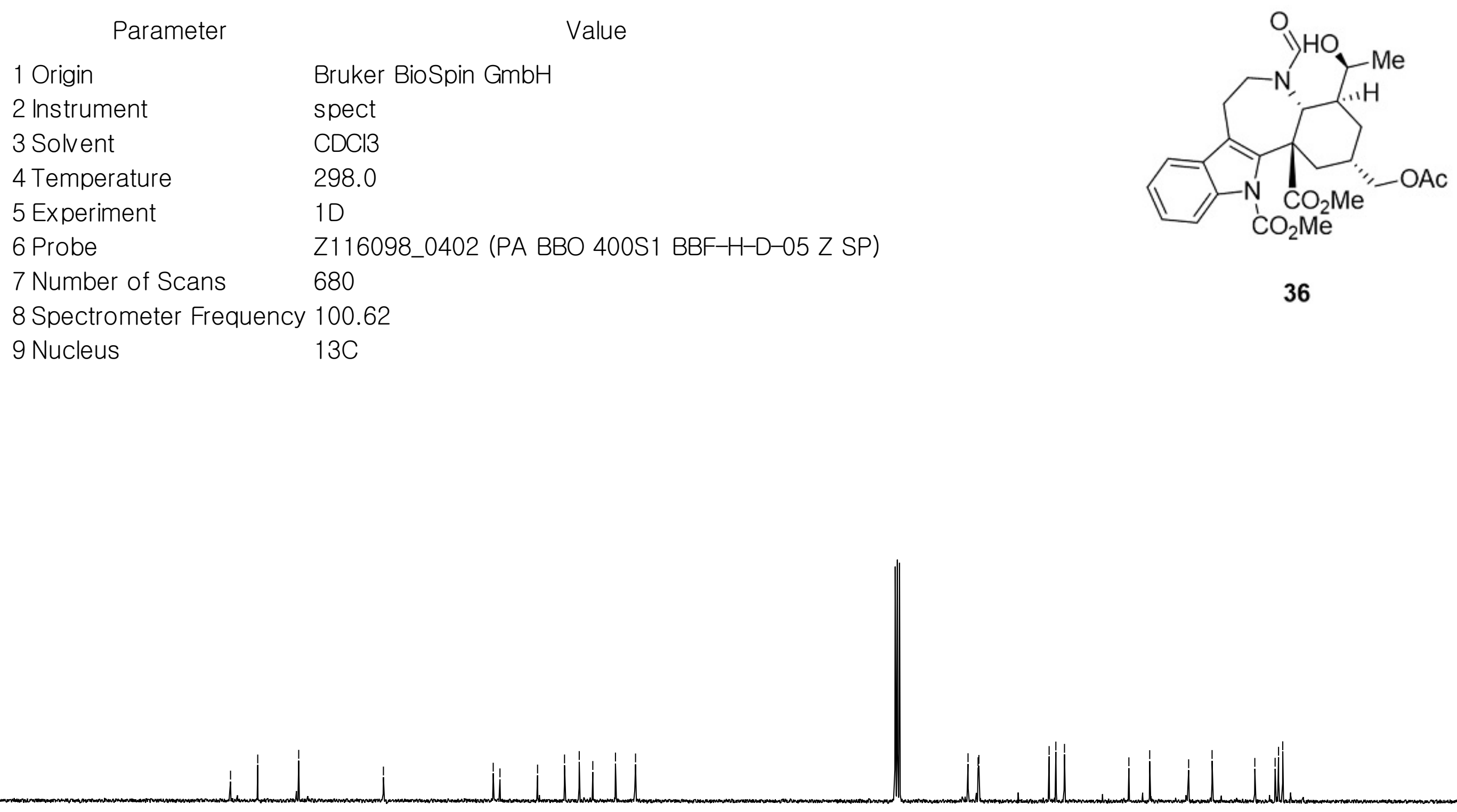


\section{Parameter}

\section{Value}

1 Origin

Varian

2 Instrument

vnmrs

3 Solvent

cdcl3

4 Temperature

25.0

5 Experiment

10

6 Probe

5mm_One_probe

7 Number of Scans

16

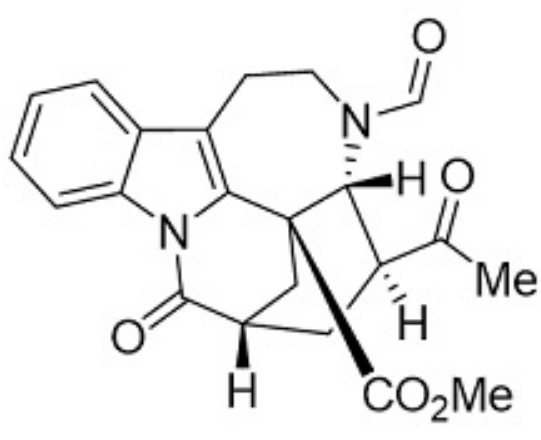

37

8 Spectrometer Frequency 599.92

9 Nucleus

$1 \mathrm{H}$

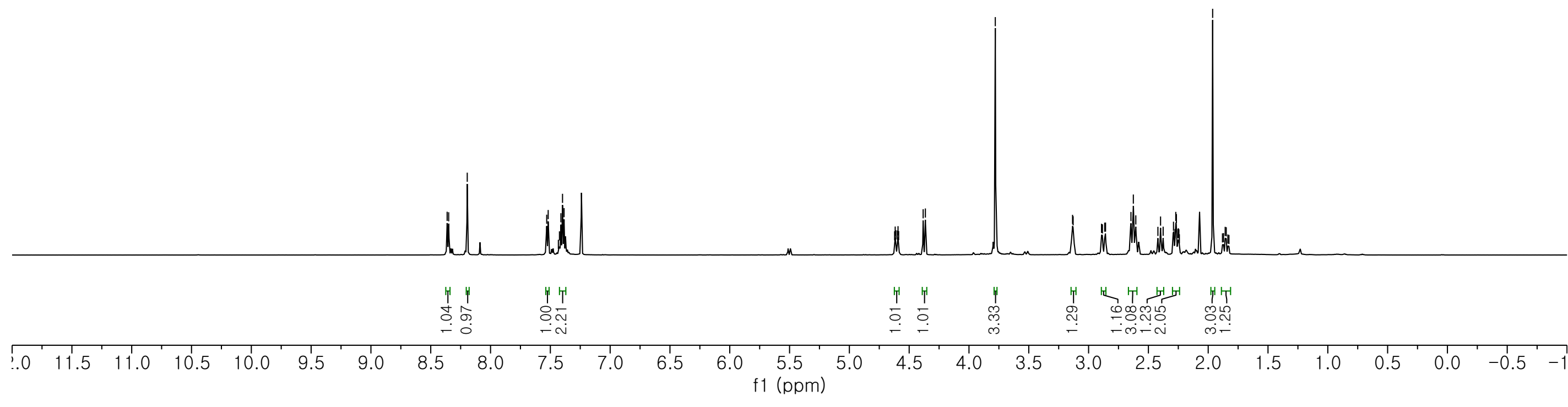


Bio-Patterned Reorganization of Alkaloids Enabled by Ring-Opening Functionalization of Tertiary Amines

\section{Parameter}

Value

1 Origin

2 Instrument

3 Solvent

4 Temperature

5 Experiment

6 Probe

7 Number of Scans

8 Spectrometer Frequency 150.87

9 Nucleus

$13 \mathrm{C}$

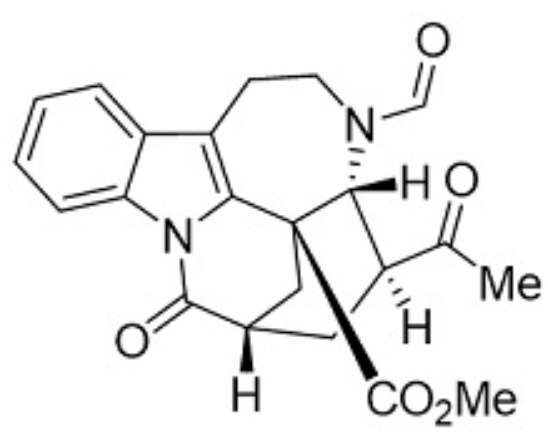

37 


\section{Parameter}

\section{Value}

\section{Origin}

2 Instrument

3 Solvent

4 Temperature

5 Experiment

6 Probe

7 Number of Scans

8 Spectrometer Frequency 400.12

9 Nucleus

spect

$\mathrm{CDCl} 3$

298.0

$1 \mathrm{D}$

16

$1 \mathrm{H}$
Bruker BioSpin $\mathrm{GmbH}$

Z116098_0402 (PA BBO 400S1 BBF-H-D-05 Z SP)

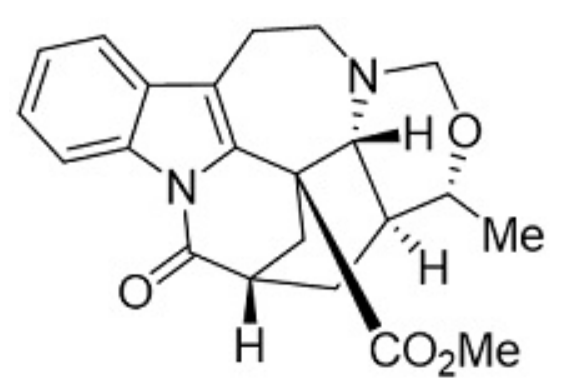

39

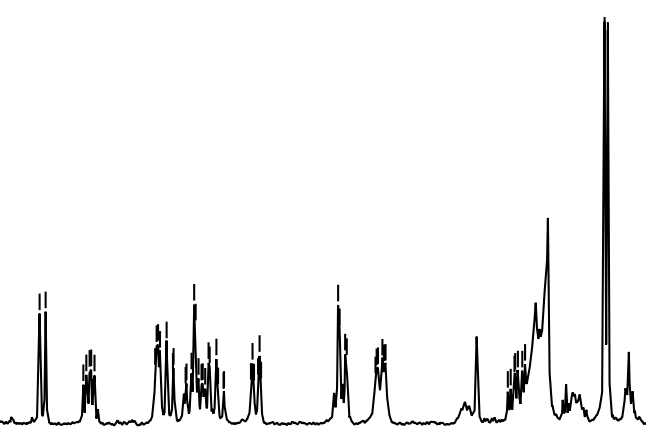

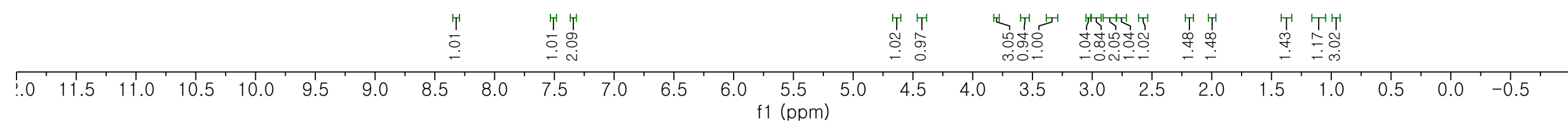


Bio-Patterned Reorganization of Alkaloids Enabled by Ring-Opening Functionalization of Tertiary Amines

Page S145 / S183

$$
\text { 睯 }
$$$$
\text { ஸा। }
$$

Value

\section{Parameter}

\section{Origin}

2 Instrument

3 Solvent

4 Temperature

5 Experiment

6 Probe

7 Number of Scans

$\begin{array}{ll}8 \text { Spectrometer Frequency } 100.62 \\ 9 \text { Nucleus } & 13 \mathrm{C}\end{array}$
Bruker BioSpin GmbH

spect

$\mathrm{CDCl} 3$

298.0

10

Z116098_0402 (PA BBO 400S1 BBF-H-D-05 Z SP) 5000

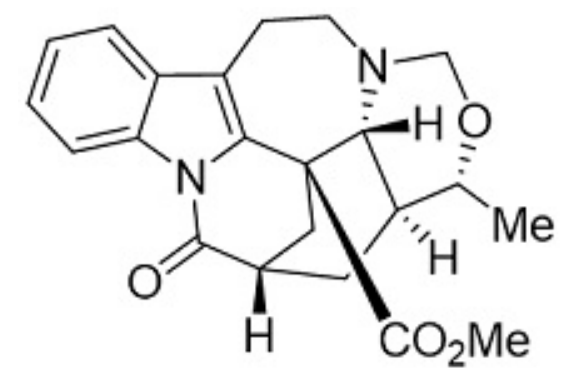

39

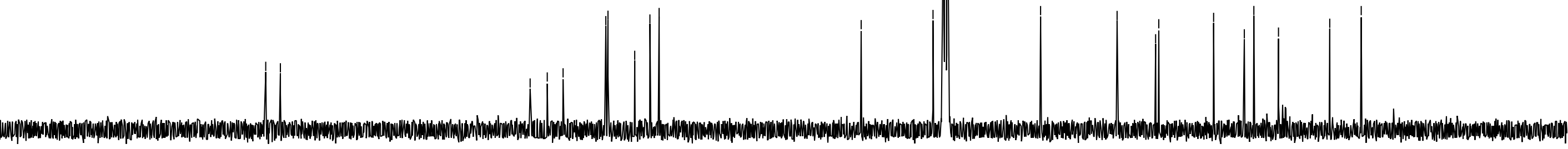


Page S146 / S183

Parameter

1 Origin

2 Instrument

3 Solvent

4 Temperature

5 Experiment

6 Probe

7 Number of Scans

8 Spectrometer Frequency 400.13

9 Nucleus

spect

$\mathrm{CDCl} 3$

295.2

10
$1 \mathrm{H}$

41

$\mathrm{H}$

\section{Value}

UXNMR, Bruker Analytische Messtechnik GmbH

5 mm Dual 13C/ 1H/Z8246/ 0025

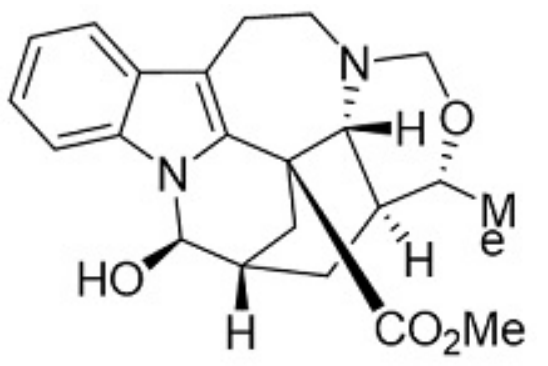

(-)-dippinine C (5) 
Bio-Patterned Reorganization of Alkaloids Enabled by Ring-Opening Functionalization of Tertiary Amines

Lim, H.; Seong, S.; Kim, Y.; Seo, S.; Han, S.*

$\begin{array}{llll}\text { II } & \text { Page S147 / S183 }\end{array}$

Parameter

Value

1 Origin

2 Instrument

3 Solvent

4 Temperature

5 Experiment

6 Probe

7 Number of Scans
Bruker BioSpin GmbH

spect

CDCl3

298.0

10

Z116098_0402 (PA BBO 400S1 BBF-H-D-05 Z SP) 5000


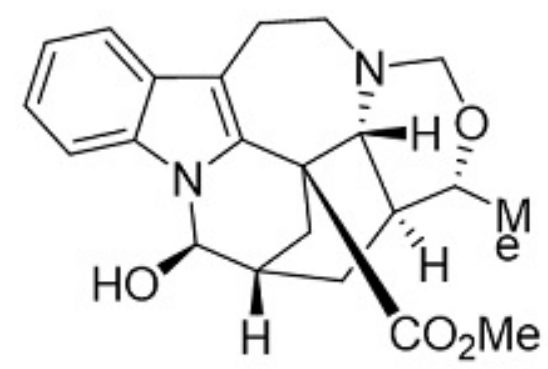

(-)-dippinine C (5)

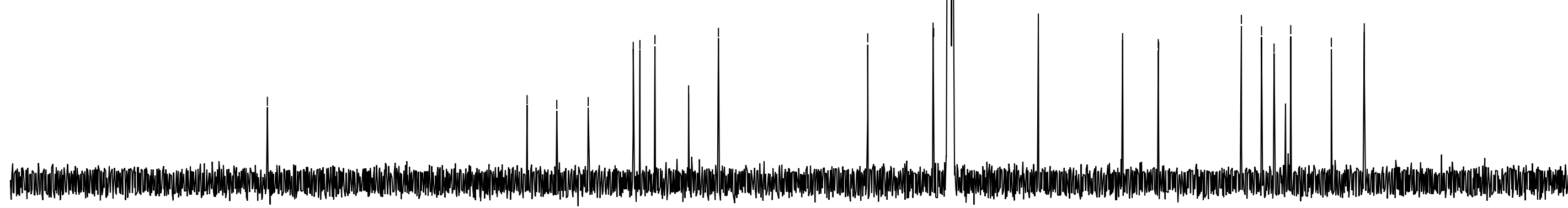




\section{Parameter}

1 Origin

2 Instrument

3 Solvent

4 Temperature

5 Experiment

6 Probe

7 Number of Scans

8 Spectrometer Frequency 400.13

9 Nucleus

$1 \mathrm{H}$

spect

$\mathrm{CDCl} 3$

292.2

10

23

\section{Value}

UXNMR, Bruker Analytische Messtechnik GmbH

$5 \mathrm{~mm}$ Dual 13C/ 1H/Z8246/ 0025

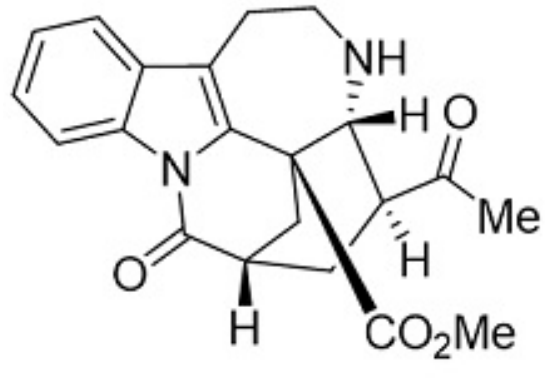

40

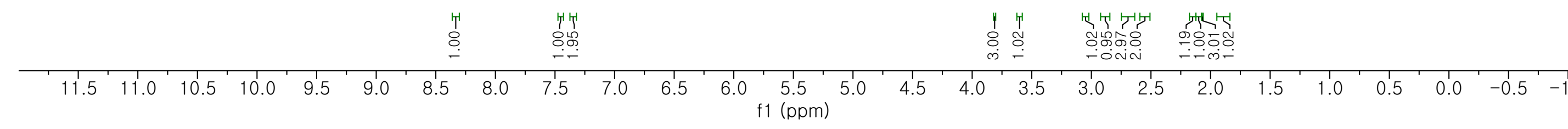


Alkaloids Enabled by Ring-Opening Functionalization of Tertiary Amines

Page S149 / S183

Parameter

Value

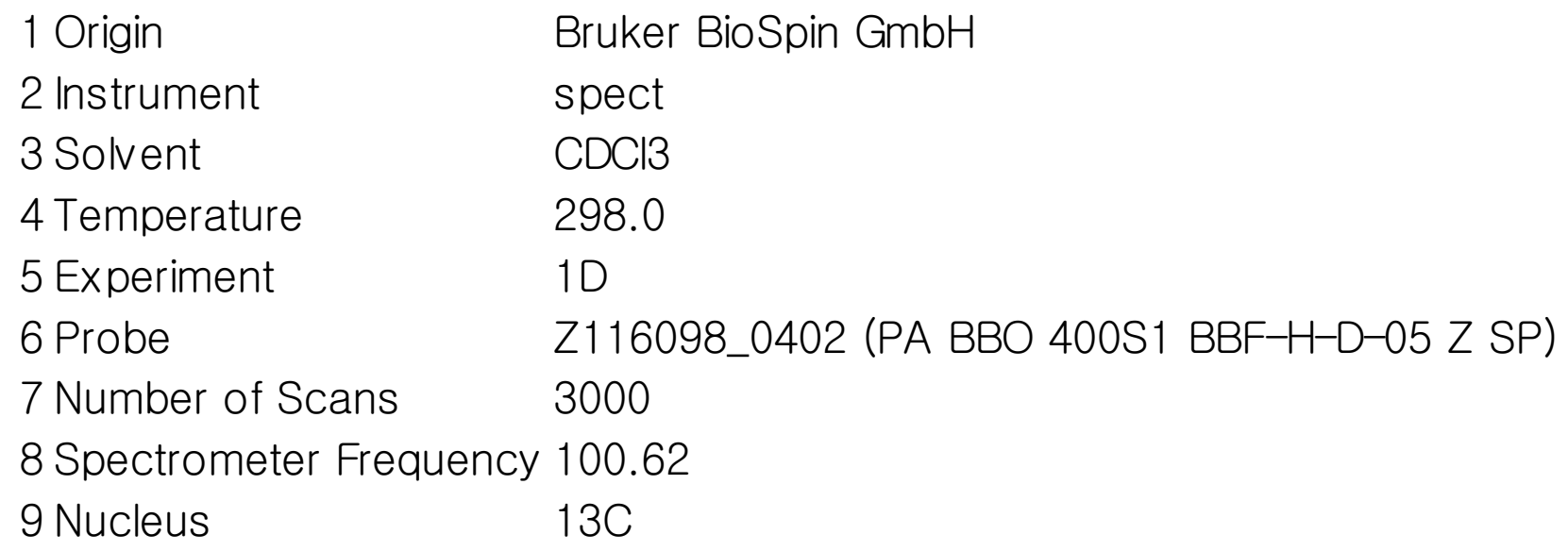

$\begin{array}{ll}8 \text { Spectrometer Frequency } 100.62 \\ 9 \text { Nucleus } & 13 \mathrm{C}\end{array}$

$\begin{array}{ll}8 \text { Spectrometer Frequency } 100.62 \\ 9 \text { Nucleus } & 13 \mathrm{C}\end{array}$

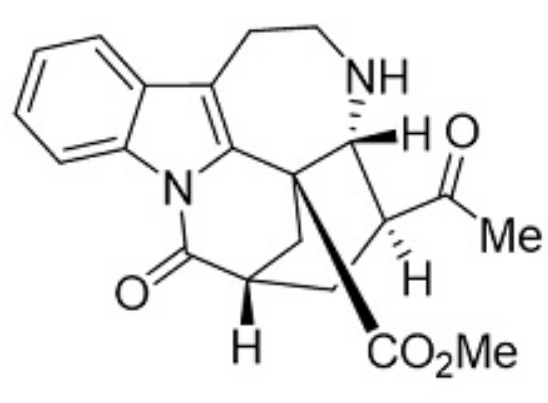

40

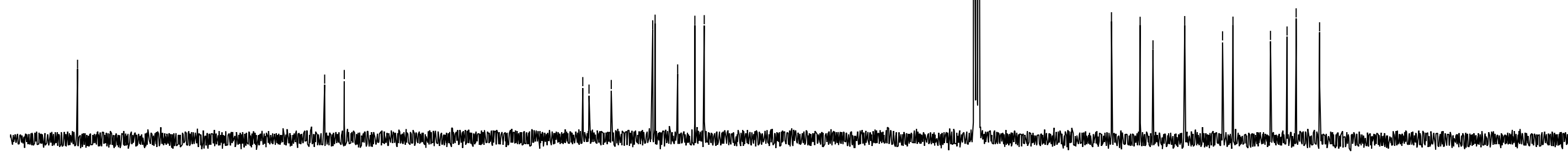


Parameter

1 Origin

2 Instrument

3 Solvent

4 Temperature

5 Experiment

6 Probe

7 Number of Scans

8 Spectrometer Frequency 400.12

9 Nucleus

spect

CDCl3

298.0

10

16
Value

Bruker BioSpin GmbH

Z116098_0402 (PA BBO 400S1 BBF-H-D-05 Z SP)

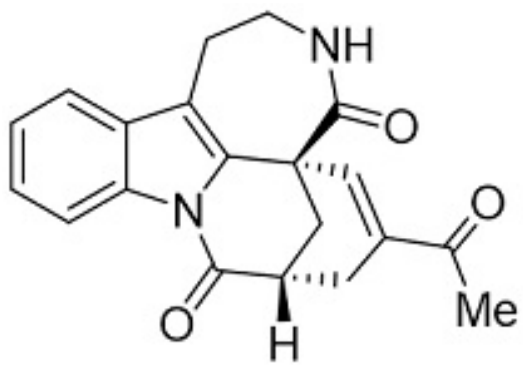

42

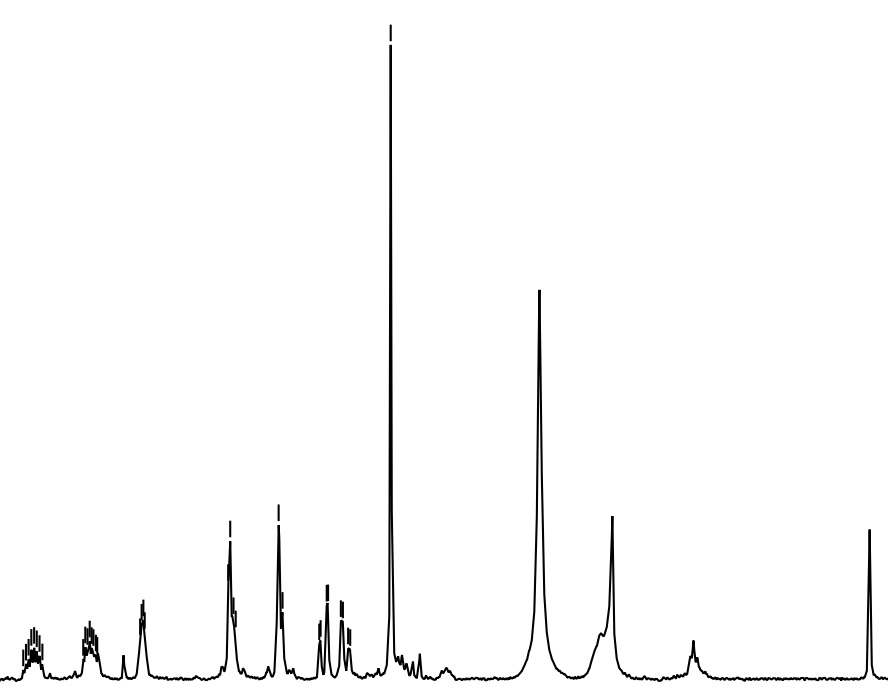

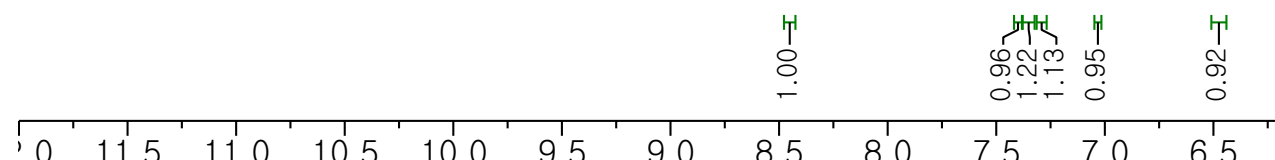

Tि

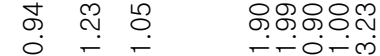

$5 \quad 8.0$


Bio-Patterned Reorganization of Alkaloids Enabled by Ring-Opening Functionalization of Tertiary Amines

\section{Parameter}

\section{Value}

\section{Origin}

2 Instrument

3 Solvent

4 Temperature

5 Experiment

6 Probe

7 Number of Scans

8 Spectrometer Frequency 100.62

9 Nucleus

$13 \mathrm{C}$

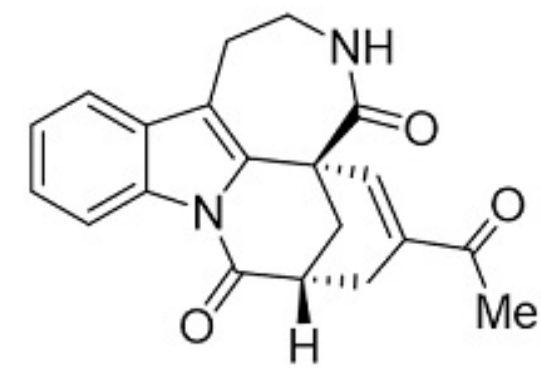

42

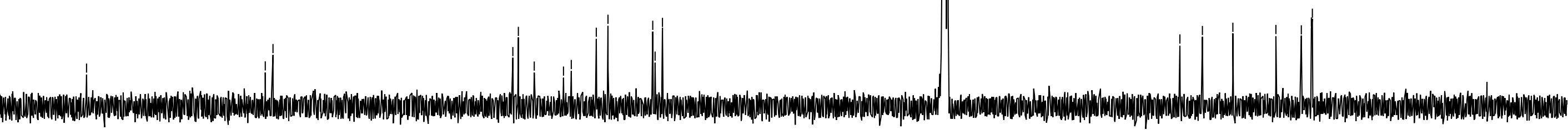




\section{Parameter}

1 Origin

2 Instrument

3 Solvent

4 Temperature

5 Experiment

6 Probe

7 Number of Scans

8 Spectrometer Frequency 400.12

9 Nucleus

spect

$\mathrm{CDCl} 3$

298.0

10

64

$1 \mathrm{H}$

\section{Value}

Bruker BioSpin GmbH

Z116098_0402 (PA BBO 400S1 BBF-H-D-05 Z SP)

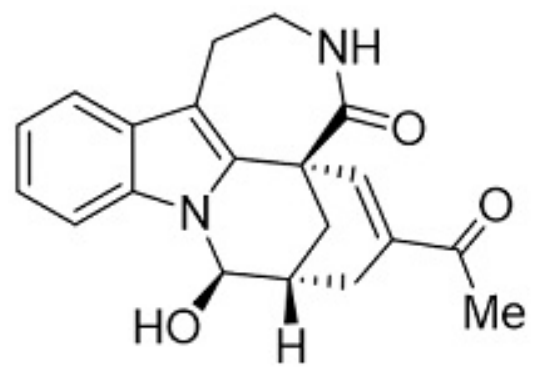

(-)-tronocarpine (6)

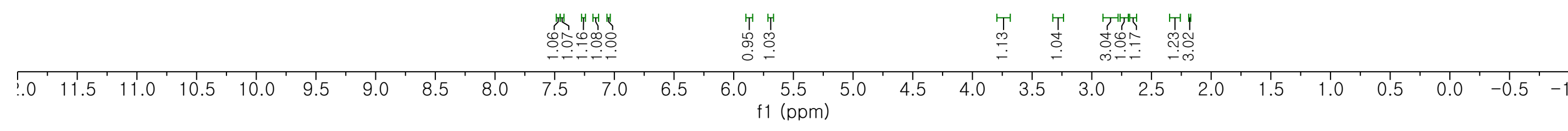


Bio-Patterned Reorganization of Alkaloids Enabled by Ring-Opening Functionalization of Tertiary Amines

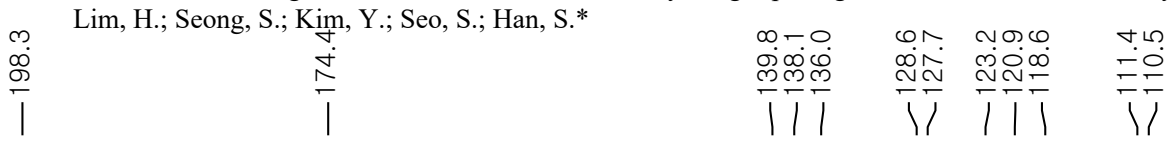

\section{Parameter}

Value

1 Origin

2 Instrument

3 Solvent

4 Temperature

5 Experiment

6 Probe

7 Number of Scans

8 Spectrometer Frequency 100.62

9 Nucleus

$13 \mathrm{C}$
|

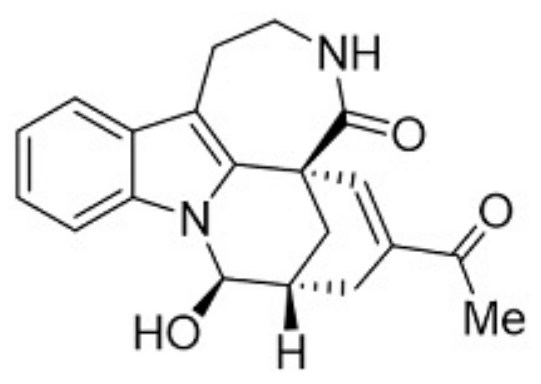

(-)-tronocarpine (6)

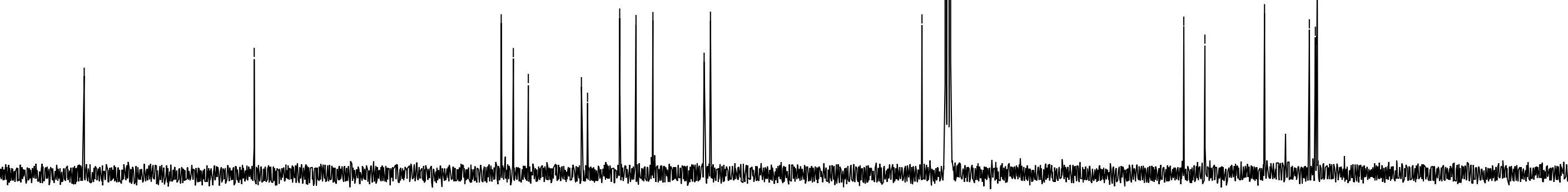


" $1 \mathrm{~mm}$

\section{Parameter}

1 Origin

2 Instrument

3 Solvent

4 Temperature

5 Experiment

6 Probe

7 Number of Scans

8 Spectrometer Frequency 500.23

9 Nucleus

$\mathrm{CDCl} 3$

298.0

10

10

$1 \mathrm{H}$

\section{Value}

Bruker BioSpin GmbH

Avance

Z151574_0070 (PI HR-BBO500S1-BBF/ H/ D-5.0-Z SP)

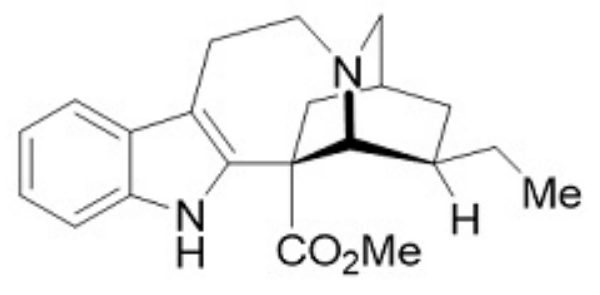

(+)-coronaridine (S11)

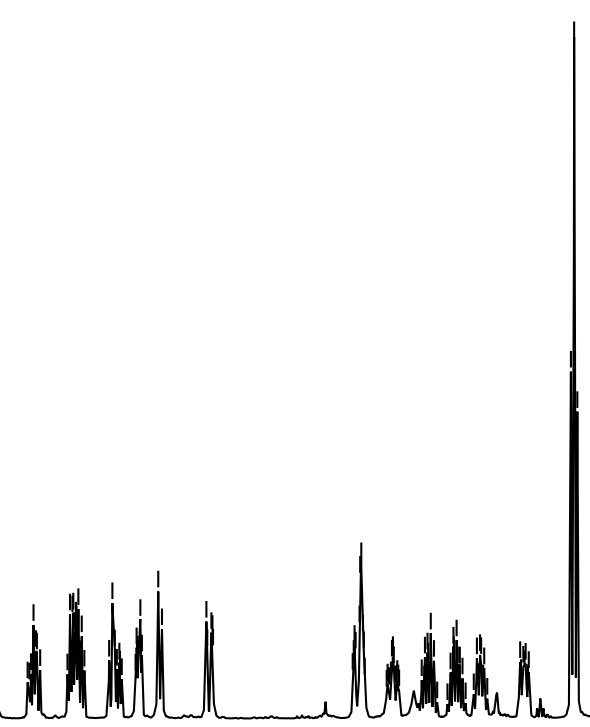

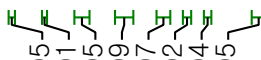

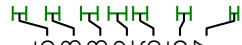

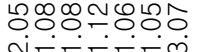

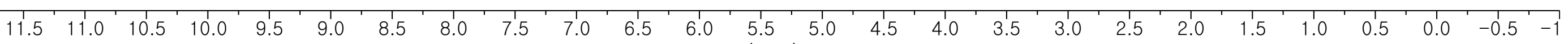




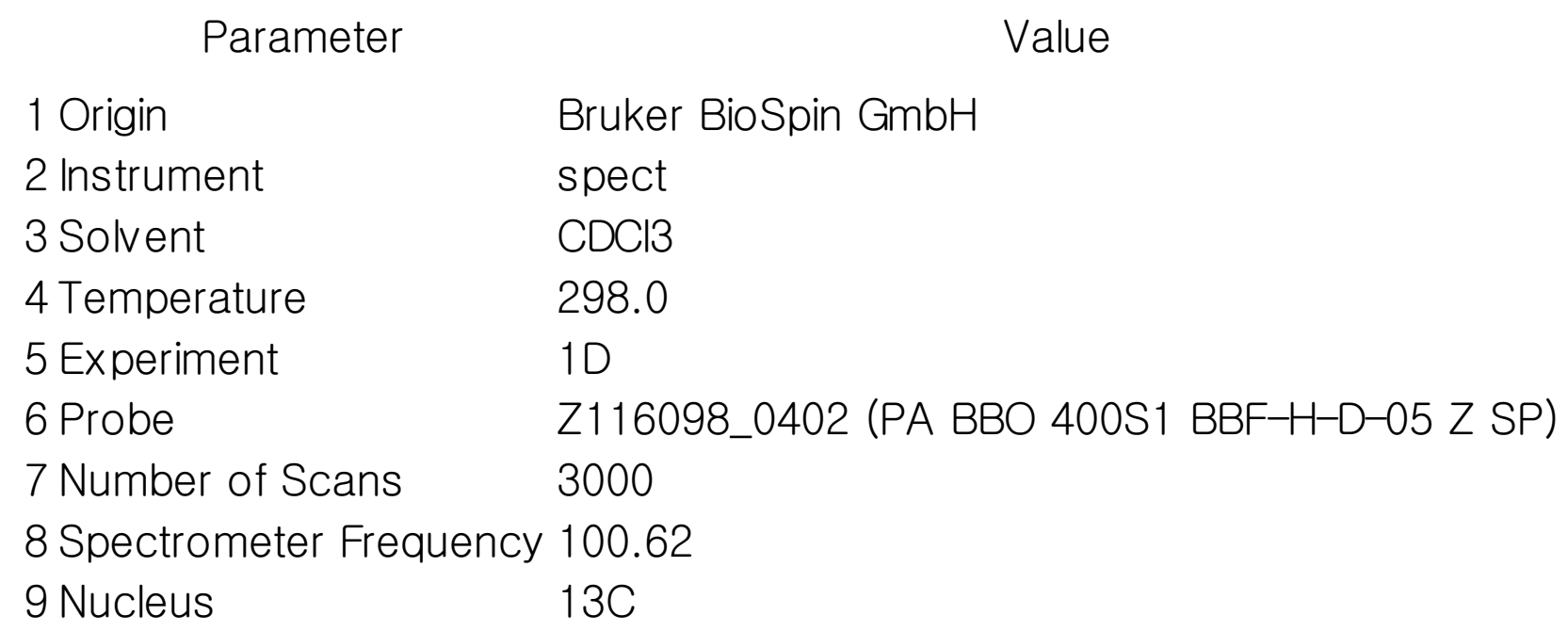

\section{Origin}

2 Instrument

3 Solvent

4 Temperature

5 Experiment

6 Probe

7 Number of Scans

8 Spectrometer Frequency 100.62

9 Nucleus

$13 \mathrm{C}$

Bruker BioSpin GmbH

spect

$\mathrm{CDCl} 3$

298.0

10

Z116098_0402 (PA BBO 400S1 BBF-H-D-05 Z SP) 3000

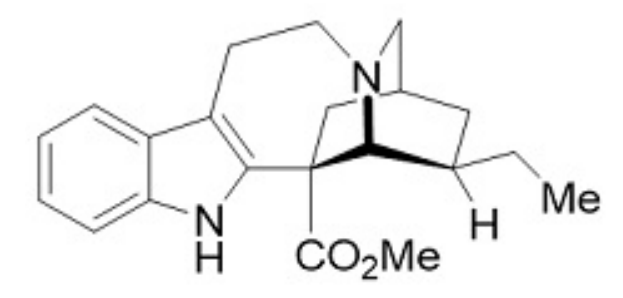

(+)-coronaridine (S11)

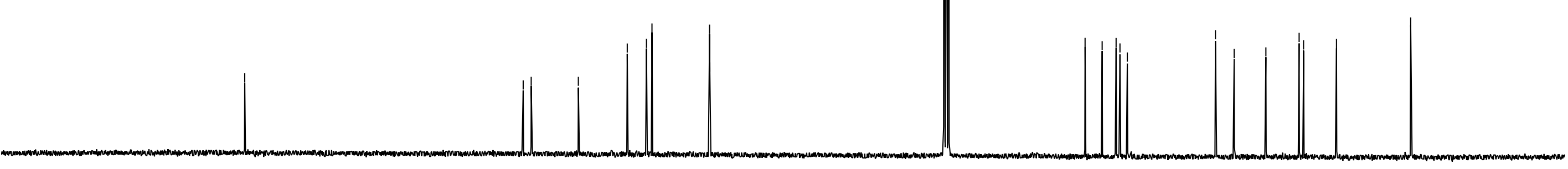




\section{"m}

\section{Parameter}

1 Origin

2 Instrument

3 Solvent

4 Temperature

5 Experiment

6 Probe

7 Number of Scans

8 Spectrometer Frequency 400.12

9 Nucleus

spect

$\mathrm{CDCl} 3$

298.0

10

16

$1 \mathrm{H}$
Value

Bruker BioSpin GmbH

Z116098_0402 (PA BBO 400S1 BBF-H-D-05 Z SP)

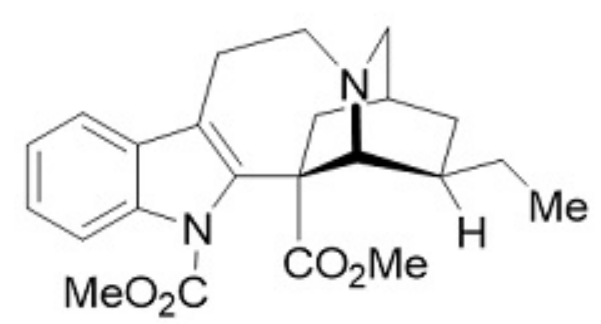

S12

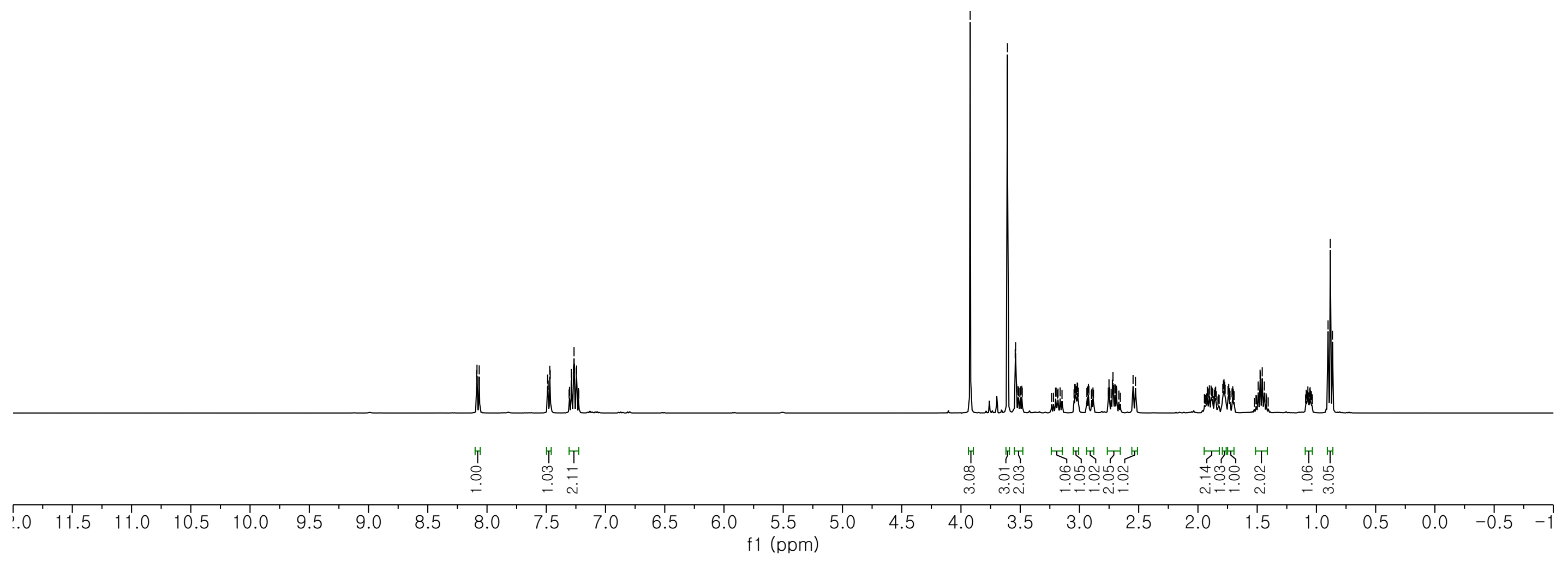




\section{Parameter}

Value

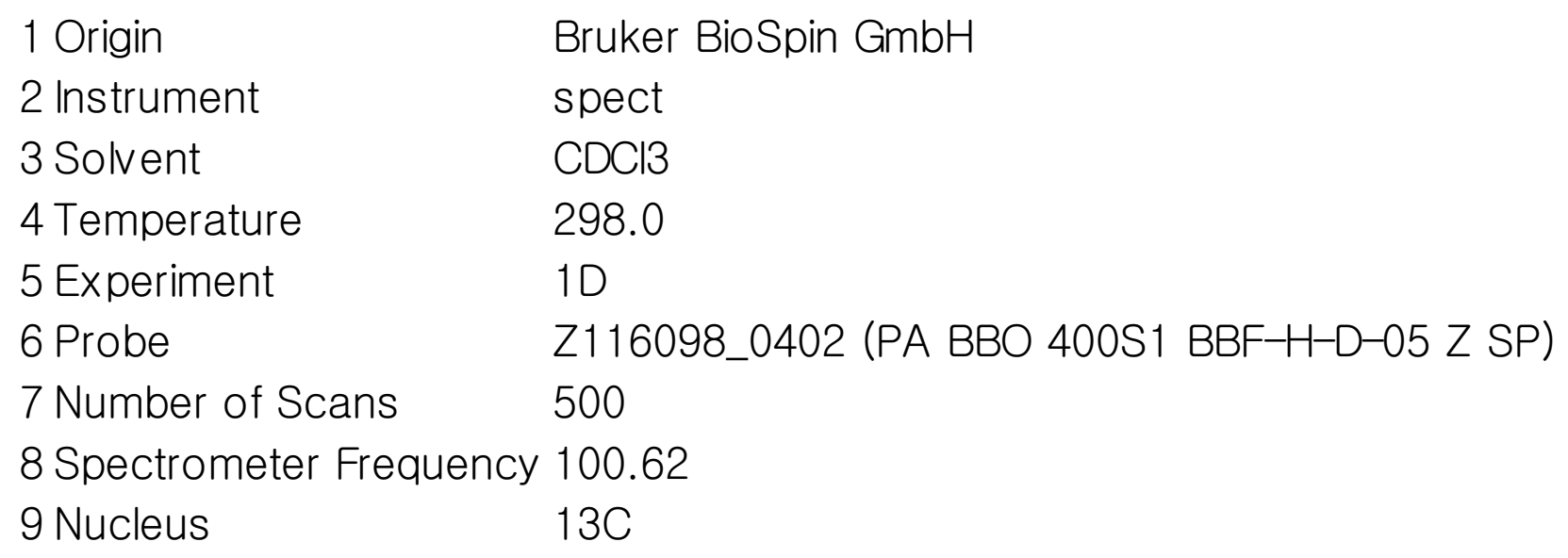

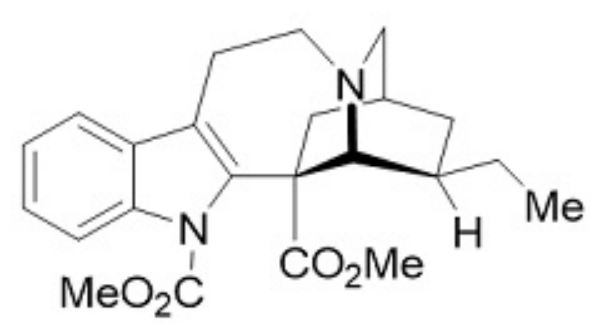

S12

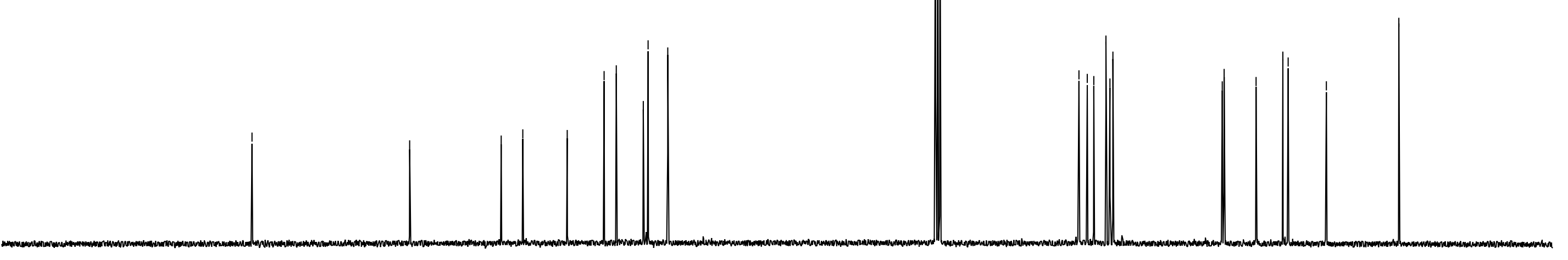




\section{Parameter}

1 Origin

2 Instrument

3 Solvent

4 Temperature

5 Experiment

6 Probe

7 Number of Scans

8 Spectrometer Frequency 500.23

9 Nucleus

$\mathrm{CDCl} 3$

298.0

10

12

$1 \mathrm{H}$
Value

Bruker BioSpin GmbH

Avance

Z151574_0070 (PI HR-BBO500S1-BBF/ H/ D-5.0-Z SP)

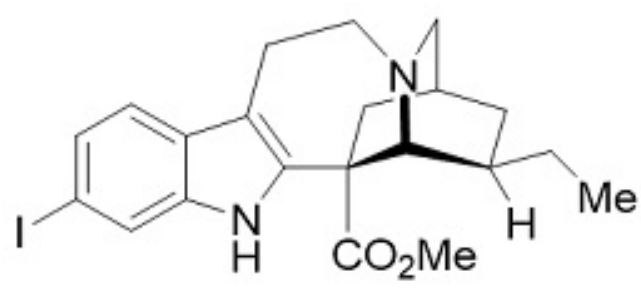

S13

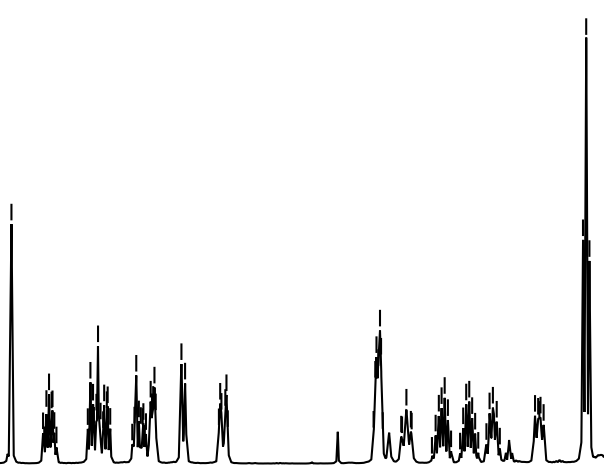

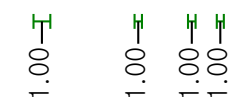

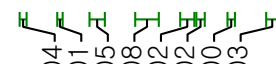

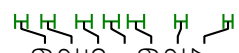

म․ำ

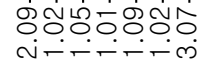

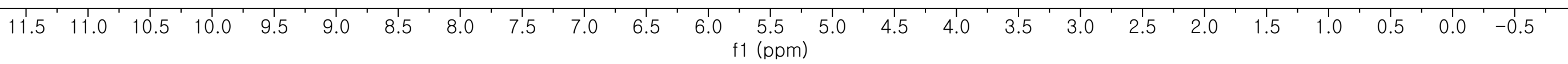




\section{Parameter}

\section{Origin}

2 Instrument

3 Solvent

4 Temperature

5 Experiment

6 Probe

7 Number of Scans

8 Spectrometer Frequency 125.80

9 Nucleus

$\mathrm{CDCl} 3$

298.2

10

67

$13 \mathrm{C}$

\section{Value}

Bruker BioSpin GmbH

Avance

Z151574_0070 (PI HR-BBO500S1-BBF/ H/ D-5.0-Z SP)

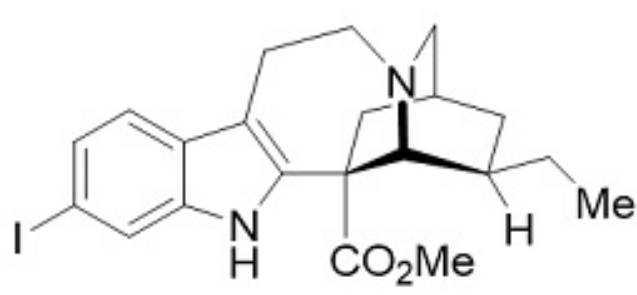

S13

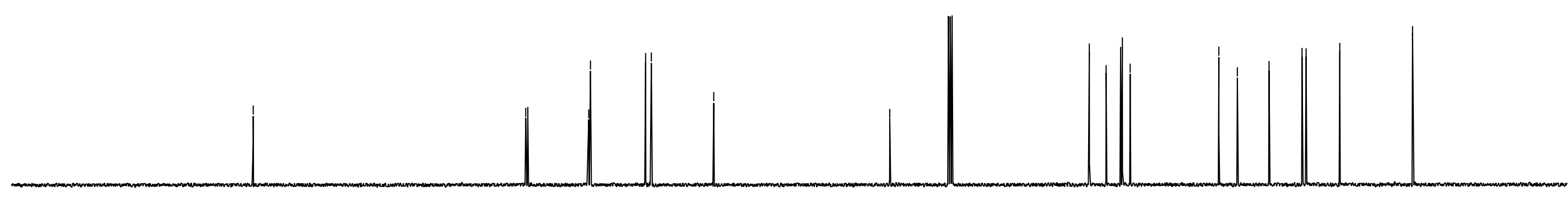




\section{Parameter}

1 Origin

2 Instrument

3 Solvent

4 Temperature

5 Experiment

6 Probe

7 Number of Scans

8 Spectrometer Frequency 500.23

9 Nucleus

$1 \mathrm{H}$

$\mathrm{CDCl} 3$

298.0

10

9
Value

Bruker BioSpin $\mathrm{GmbH}$

Avance

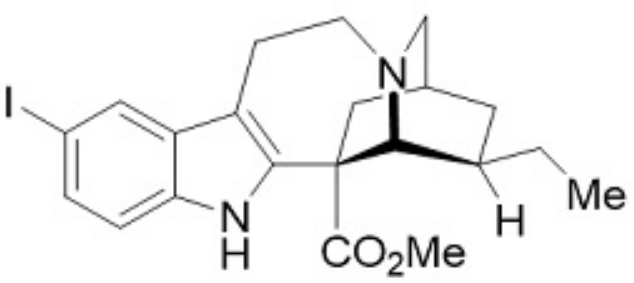

S14

Z151574_0070 (PI HR-BBO500S1-BBF/ H/ D-5.0-Z SP)

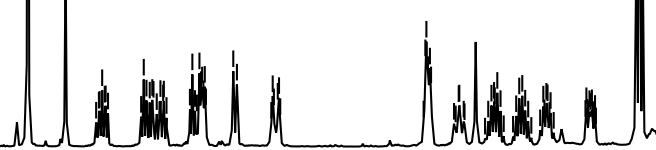

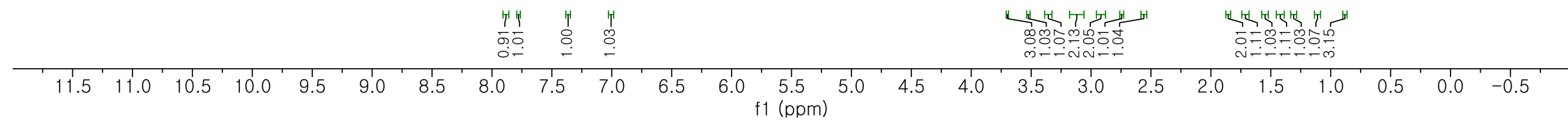


Parameter

\section{Origin}

2 Instrument

3 Solvent

4 Temperature

5 Experiment

6 Probe

7 Number of Scans

8 Spectrometer Frequency 125.80

9 Nucleus

$\mathrm{CDCl} 3$

298.2

10

322

$13 \mathrm{C}$
Value

Bruker BioSpin GmbH

Avance

Z151574_0070 (PI HR-BBO500S1-BBF/ H/ D-5.0-Z SP)

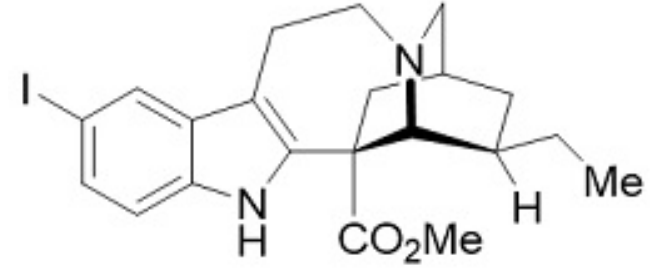

S14

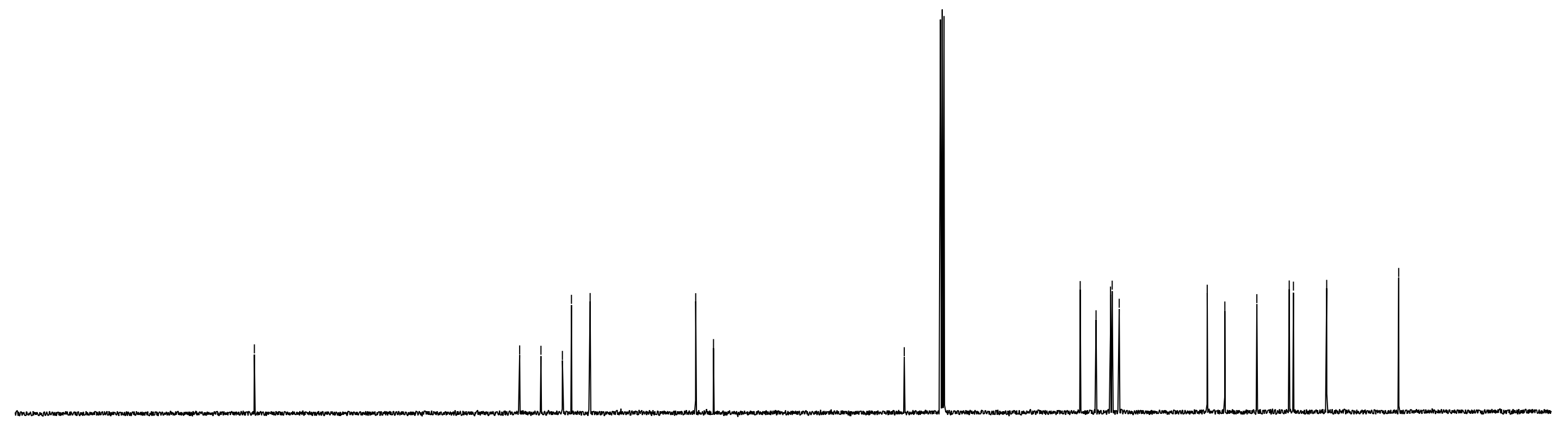

0

200

190

180

170

160

150

140

130

120

$110 \quad 100$

1 (ppm)

$90 \quad 80$

60

50

40

30

20 


\section{Parameter}

1 Origin

2 Instrument

3 Solvent

4 Temperature

5 Experiment

6 Probe

7 Number of Scans

8 Spectrometer Frequency 400.12

9 Nucleus

spect

$\mathrm{CDCl} 3$

298.0

10

16

$1 \mathrm{H}$

\section{Value}

Bruker BioSpin GmbH

Z116098_0402 (PA BBO 400S1 BBF-H-D-05 Z SP)

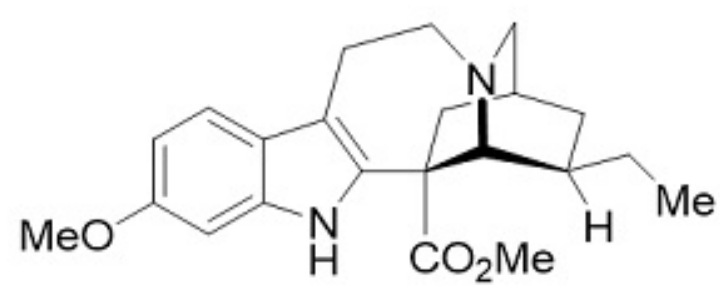

(+)-isovoacangine (44)

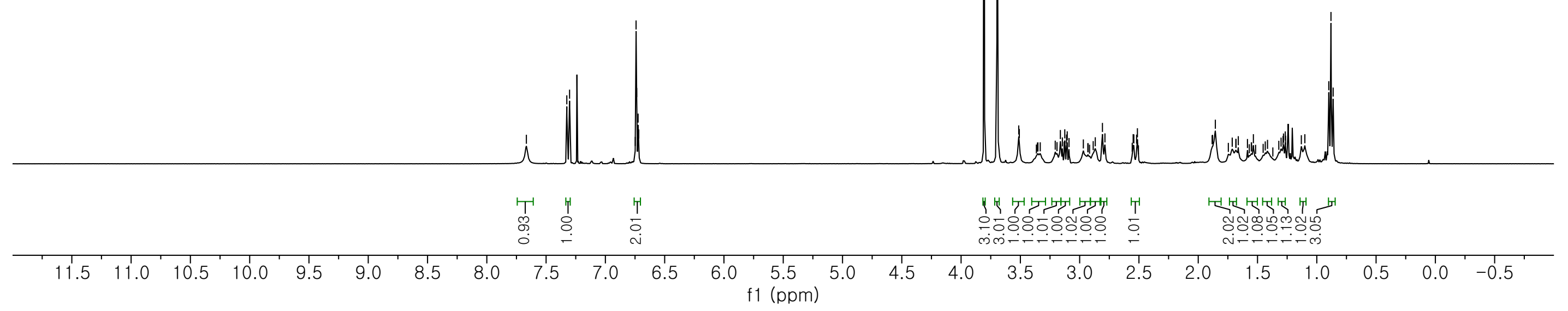




\section{Parameter}

Value

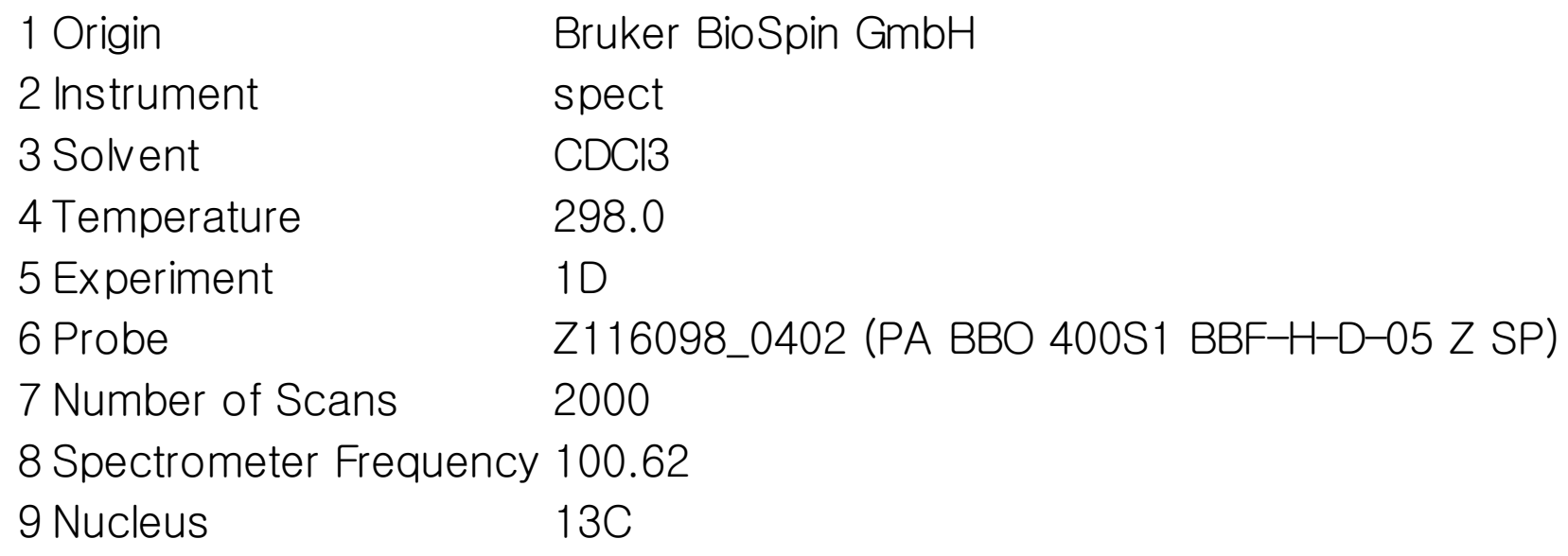

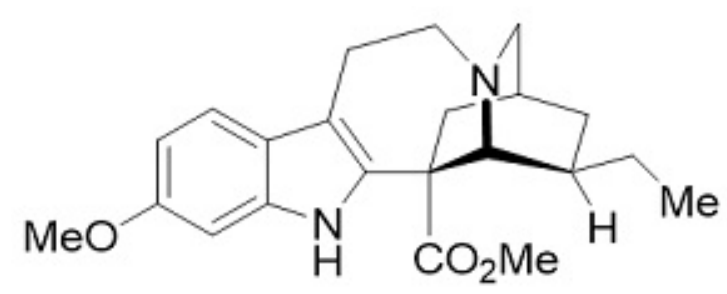

(+)-isovoacangine (44) 
Parameter

\section{Origin}

2 Instrument

3 Solvent

4 Temperature

5 Experiment

6 Probe

7 Number of Scans

8 Spectrometer Frequency 500.23

9 Nucleus

CDCl3

298.0

10

12

$1 \mathrm{H}$
Value

Bruker BioSpin GmbH

Avance

Z151574_0070 (PI HR-BBO500S1-BBF/ H/ D-5.0-Z SP)

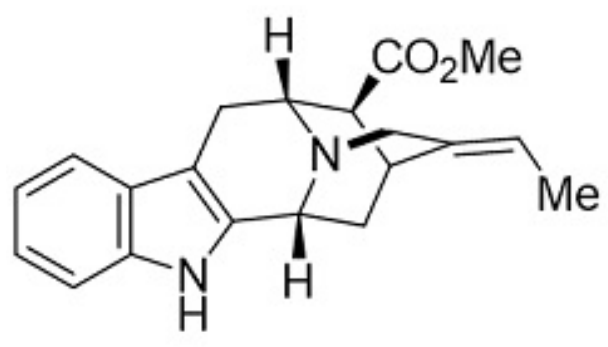

(-)-16-epi-pericyclivine (7)

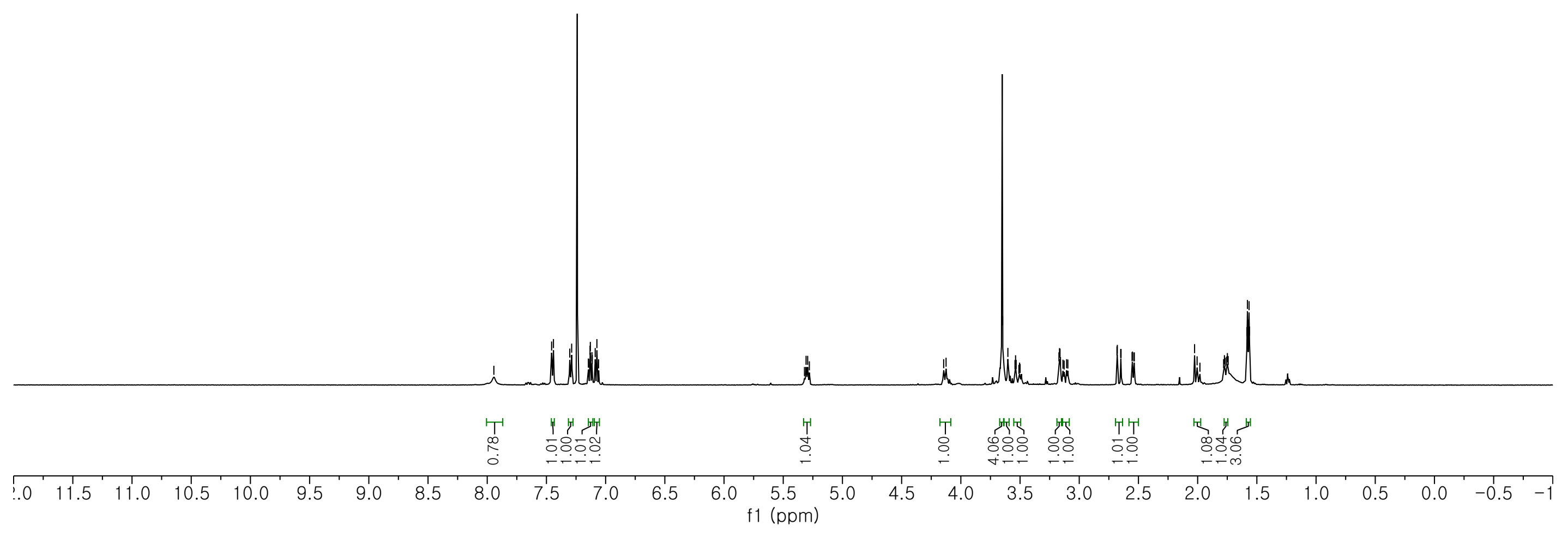




\section{Parameter}

Value

$\begin{array}{ll}1 \text { Origin } & \text { Varian } \\ 2 \text { Instrument } & \text { vnmrs } \\ \text { 3 Solvent } & \text { cdcl3 } \\ \text { 4 Temperature } & 25.0 \\ 5 \text { Experiment } & 10 \\ 6 \text { Probe } & 5 \mathrm{~mm} \text { One_probe } \\ 7 \text { Number of Scans } & 108 \\ 8 \text { Spectrometer Frequency } & 150.86 \\ 9 \text { Nucleus } & 13 \mathrm{C}\end{array}$

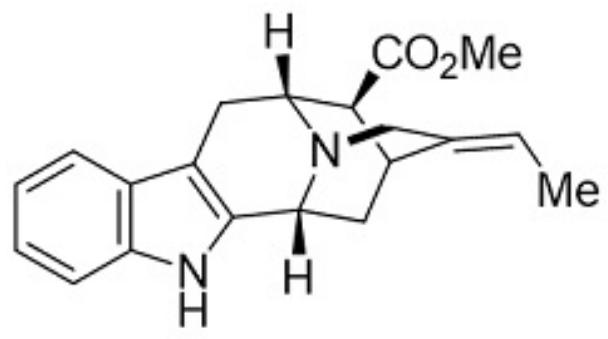

(-)-16-epi-pericyclivine (7)

$13 \mathrm{C}$ 
Bio-Patterned Reorganization of Alkaloids Enabled by Ring-Opening Functionalization of Tertiary Amines

Lim, H.; Seong, S.; Kim, Y.; Seo, S.; Han, S.*

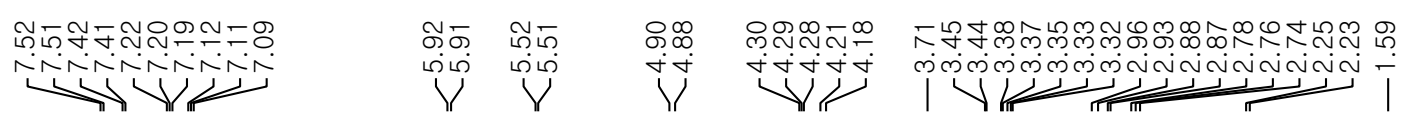

\section{Parameter}

\section{Value}

1 Origin

Varian

2 Instrument

vnmrs

3 Solvent

cdcl3

4 Temperature

25.0

5 Experiment

$1 \mathrm{D}$

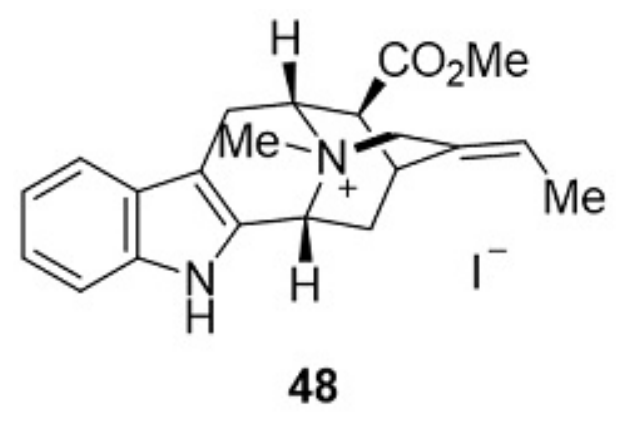

6 Probe

5mm_One_probe

7 Number of Scans

3

8 Spectrometer Frequency 599.88

9 Nucleus

$1 \mathrm{H}$

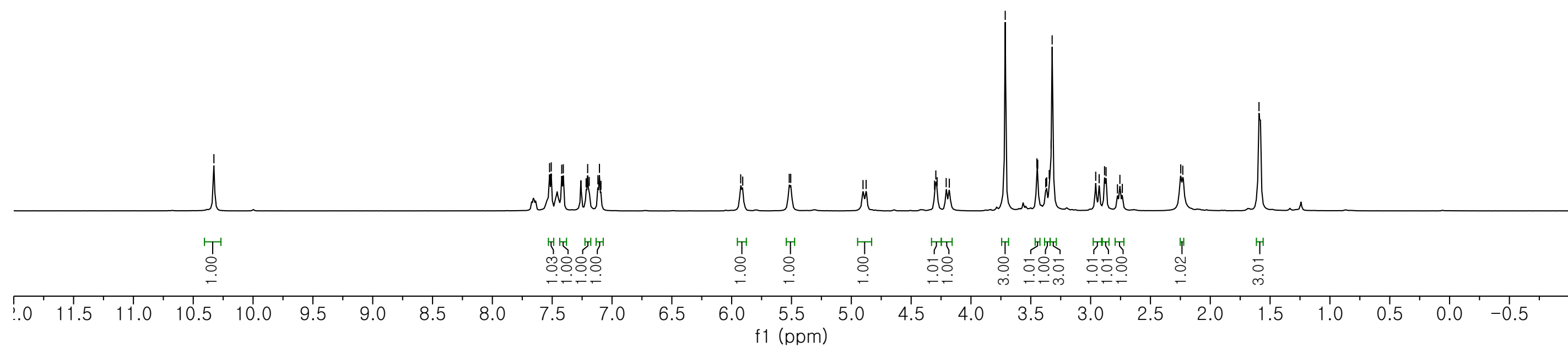




\section{Parameter}

Value

\section{Origin}

2 Instrument

Varian

3 Solvent

vnmrs

cdcl3

25.0

4 Temperature

5 Experiment

6 Probe

7 Number of Scans

8 Spectrometer Frequency 150.86

9 Nucleus

$1 \mathrm{D}$

5mm_One_probe

464

$13 \mathrm{C}$

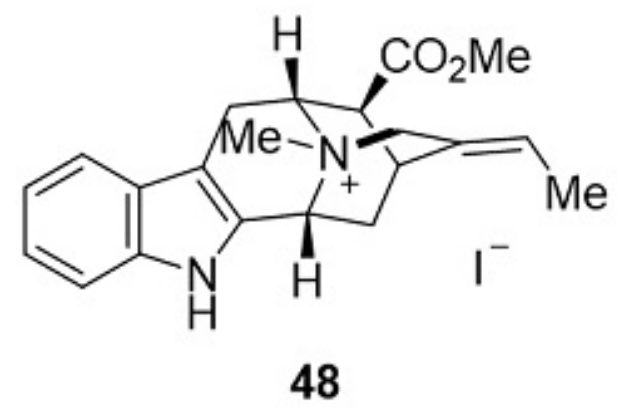

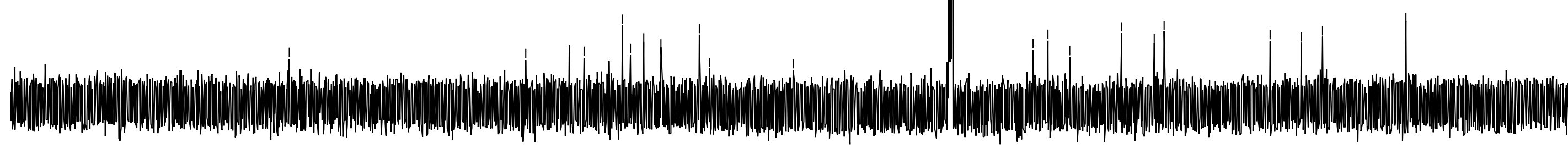


Bio-Patterned Reorganization of Alkaloids Enabled by Ring-Opening Functionalization of Tertiary Amines Lim, H.; Seong, S.; Kim, Y.; Seo, S.; Han, S.*

\section{Parameter}

1 Origin

2 Instrument

3 Solvent

4 Temperature

5 Experiment

6 Probe

7 Number of Scans

8 Spectrometer Frequency 500.23

9 Nucleus

$1 \mathrm{H}$

$\mathrm{CDCl} 3$

298.0

10

11
Value

Bruker BioSpin GmbH

Avance

Z151574_0070 (PI HR-BBO500S1-BBF/ H/ D-5.0-Z SP)

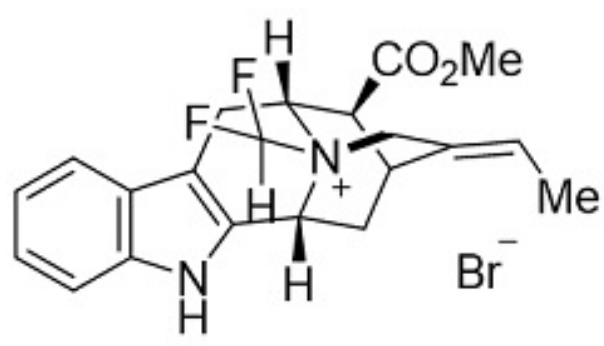

S16

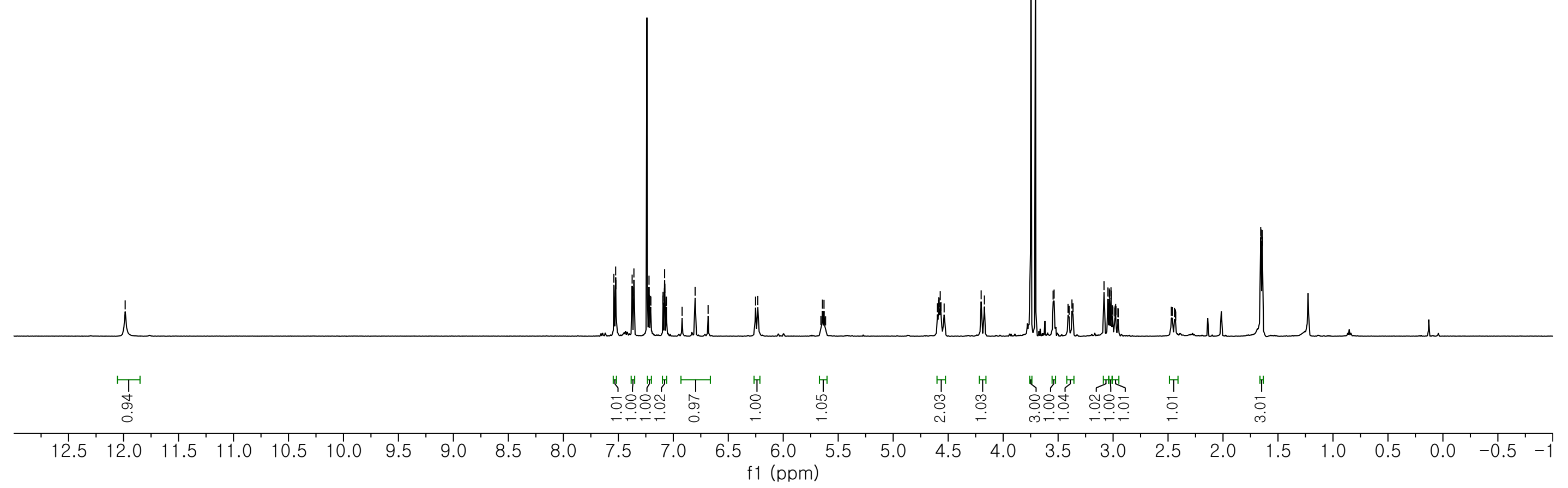




\section{Parameter}

\section{Value}

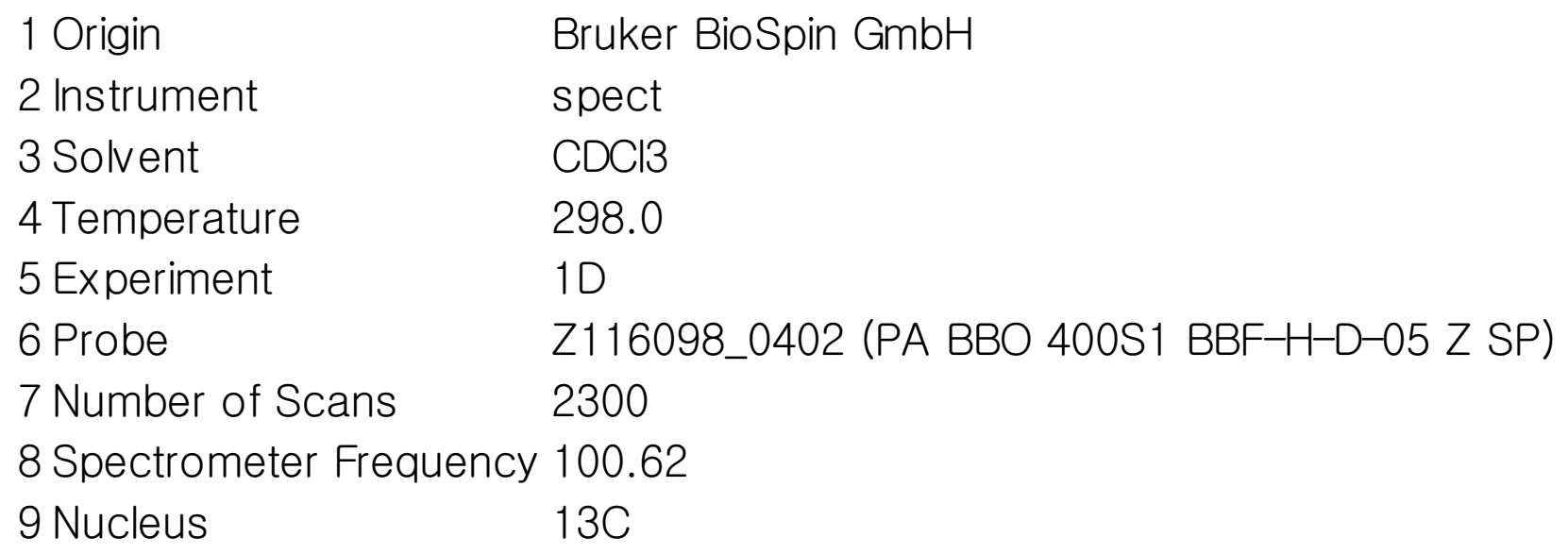

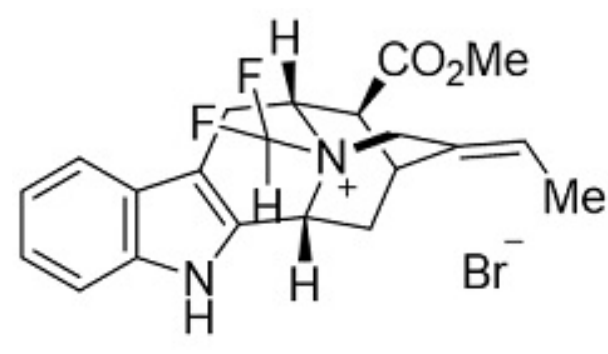

S16 


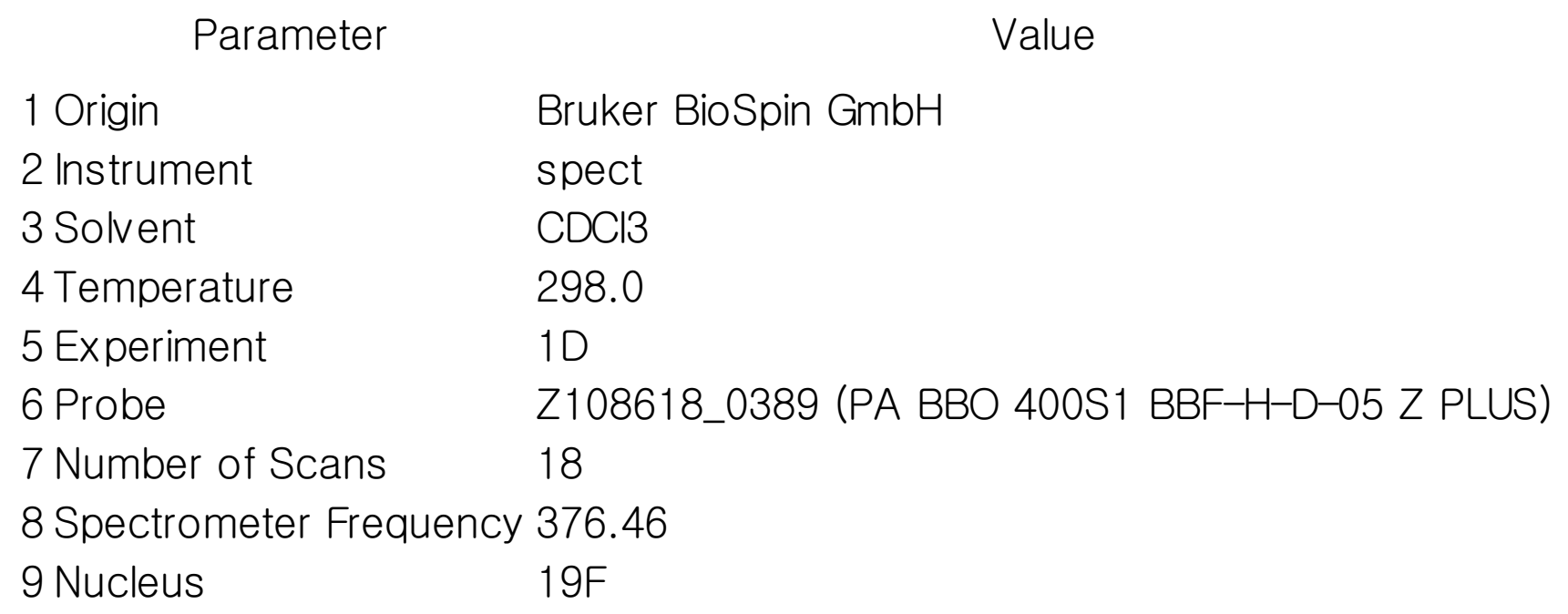




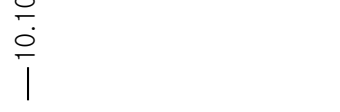

Parameter

Value

\section{Origin}

2 Instrument

3 Solvent

4 Temperature

5 Experiment

6 Probe

7 Number of Scans

8 Spectrometer Frequency 500.23

9 Nucleus

CDCl3

298.0

10 5

$1 \mathrm{H}$
Bruker BioSpin $\mathrm{GmbH}$

Avance

Z151574_0070 (PI HR-BBO500S1-BBF/ H/ D-5.0-Z SP)
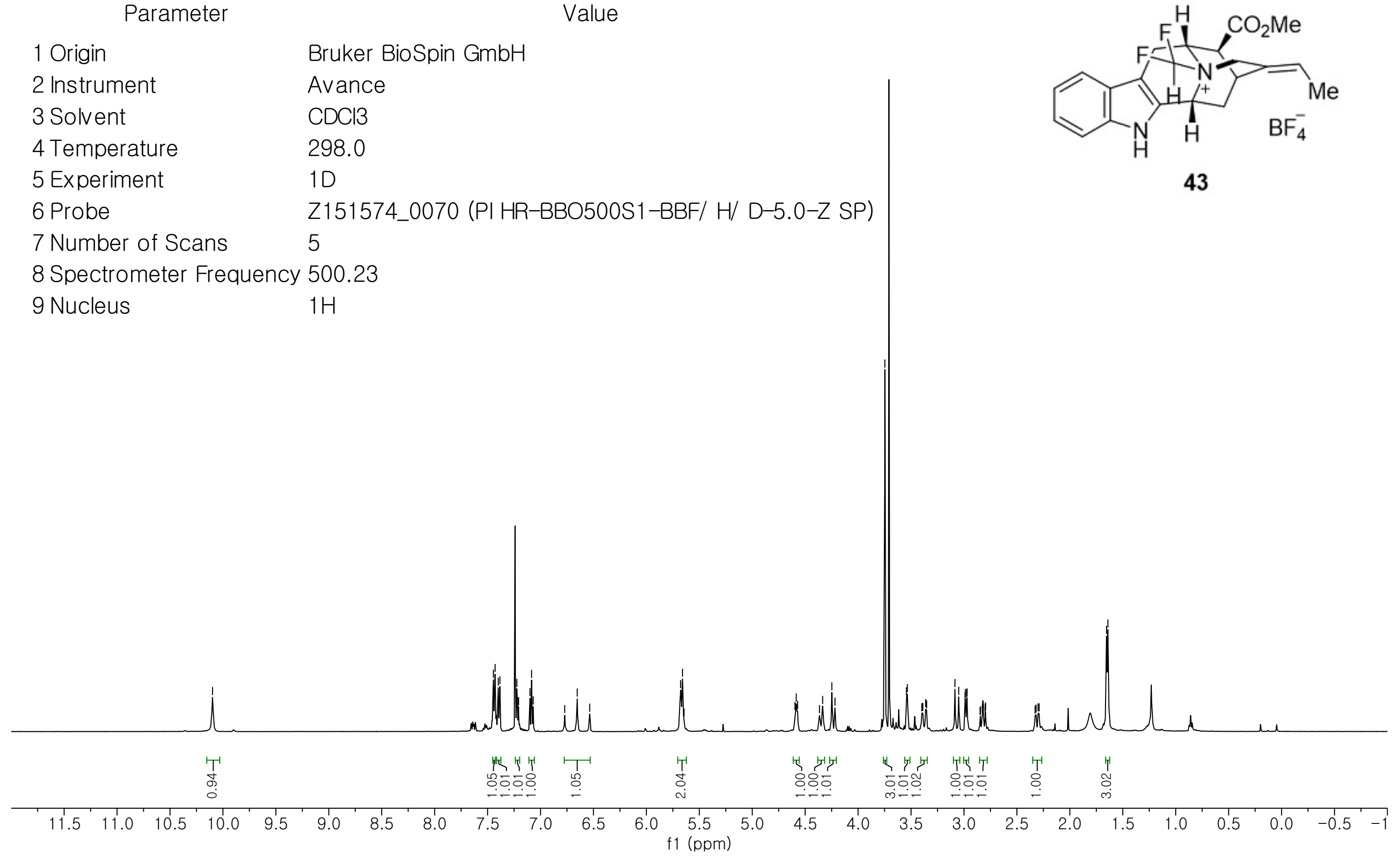


\section{Parameter}

\section{Origin}

2 Instrument

3 Solvent

4 Temperature

5 Experiment

6 Probe

7 Number of Scans

8 Spectrometer Frequency 125.80

9 Nucleus

$\mathrm{CDCl} 3$

298.2

10 249

$13 \mathrm{C}$
Value

Bruker BioSpin $\mathrm{GmbH}$

Avance

Z151574_0070 (PI HR-BBO500S1-BBF/ H/ D-5.0-Z SP)

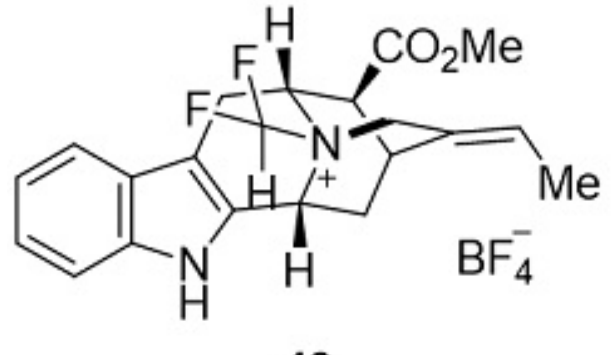

43 


\section{Parameter}

1 Origin

2 Instrument

3 Solvent

4 Temperature

5 Experiment

6 Probe

7 Number of Scans

8 Spectrometer Frequency 470.64

9 Nucleus

$\mathrm{CDCl} 3$

298.0

10

16

$19 F$
Value

Bruker BioSpin GmbH

Avance

Z151574_0070 (PI HR-BBO500S1-BBF/ H/ D-5.0-Z SP)

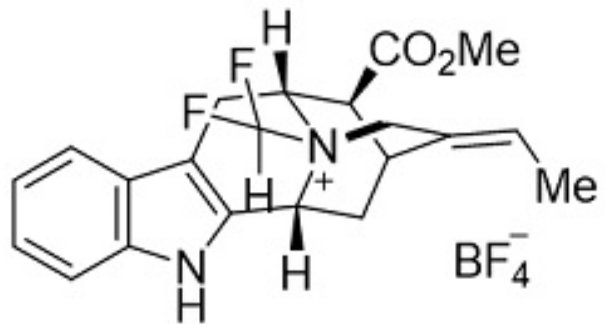

43

\section{l.}




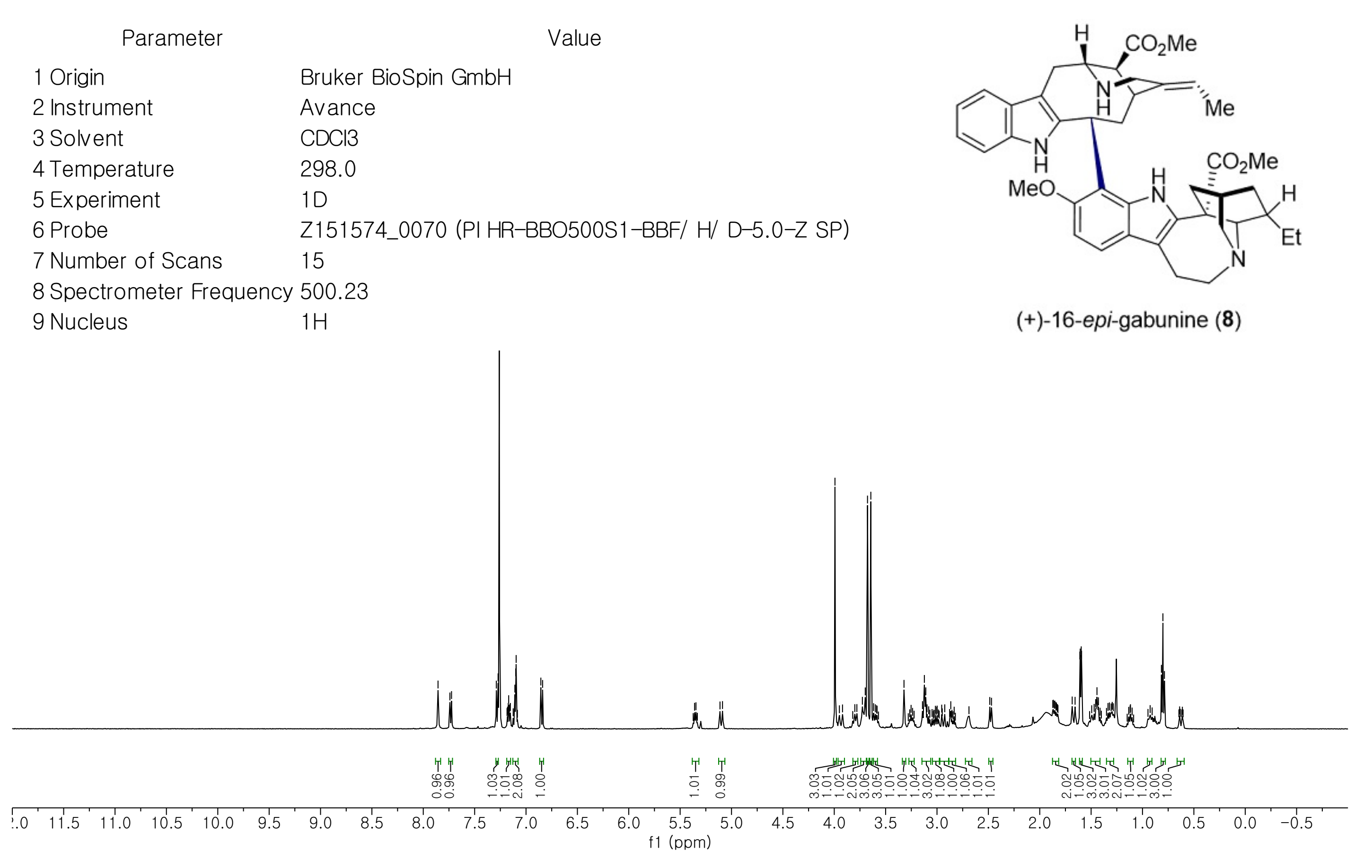


Parameter

Value

\section{Origin}

2 Instrument

3 Solvent

4 Temperature

5 Experiment

6 Probe

7 Number of Scans

8 Spectrometer Frequency 125.80

9 Nucleus

$13 \mathrm{C}$

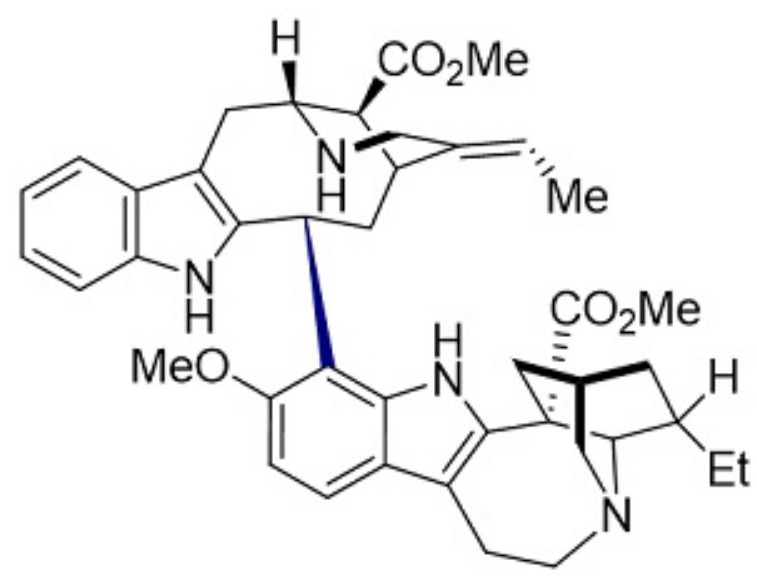

(+)-16-epi-gabunine (8)
Bruker BioSpin GmbH

Avance

CDCl3

298.2

10

Z151574_0070 (PI HR-BBO500S1-BBF/ H/ D-5.0-Z \$P) 1200

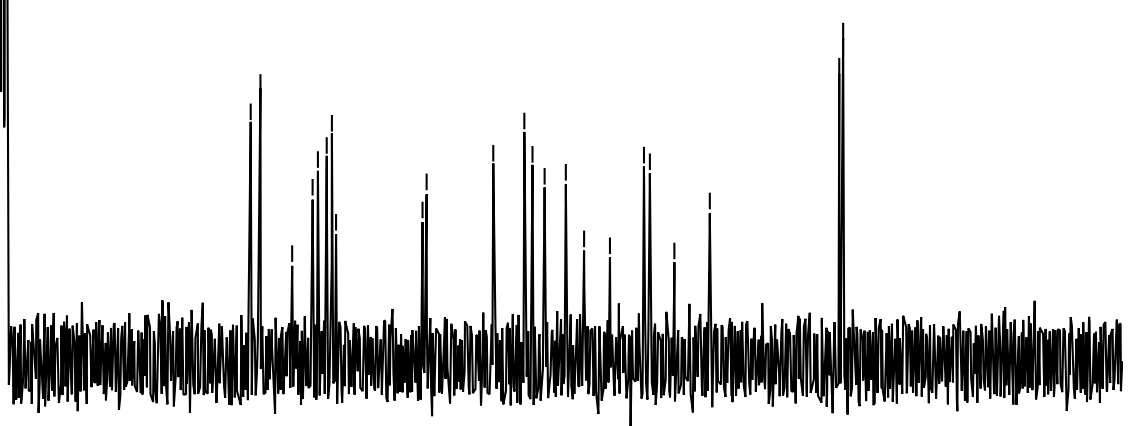




\section{Parameter Value}
1 Origin
Bruker BioSpin GmbH
2 Instrument
3 Solvent
Avance
CDCl3
4 Temperature
298.0
5 Experiment
$1 \mathrm{D}$
6 Probe
Z151574_0070 (PI HR-BBO500S1-BBF/ H/ D-5.0-Z SP)
7 Number of Scans
26
8 Spectrometer Frequency 500.23
9 Nucleus
$1 \mathrm{H}$

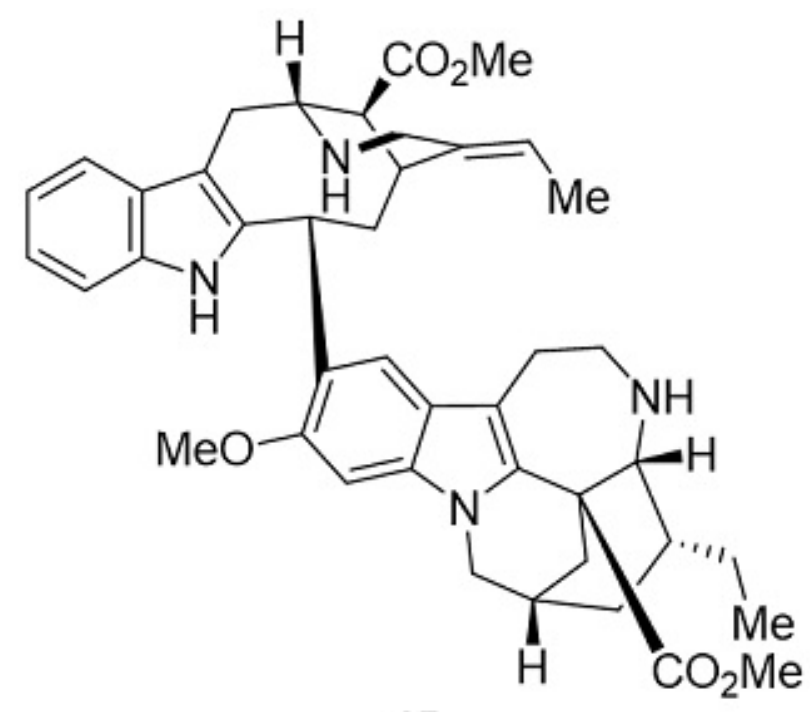

45

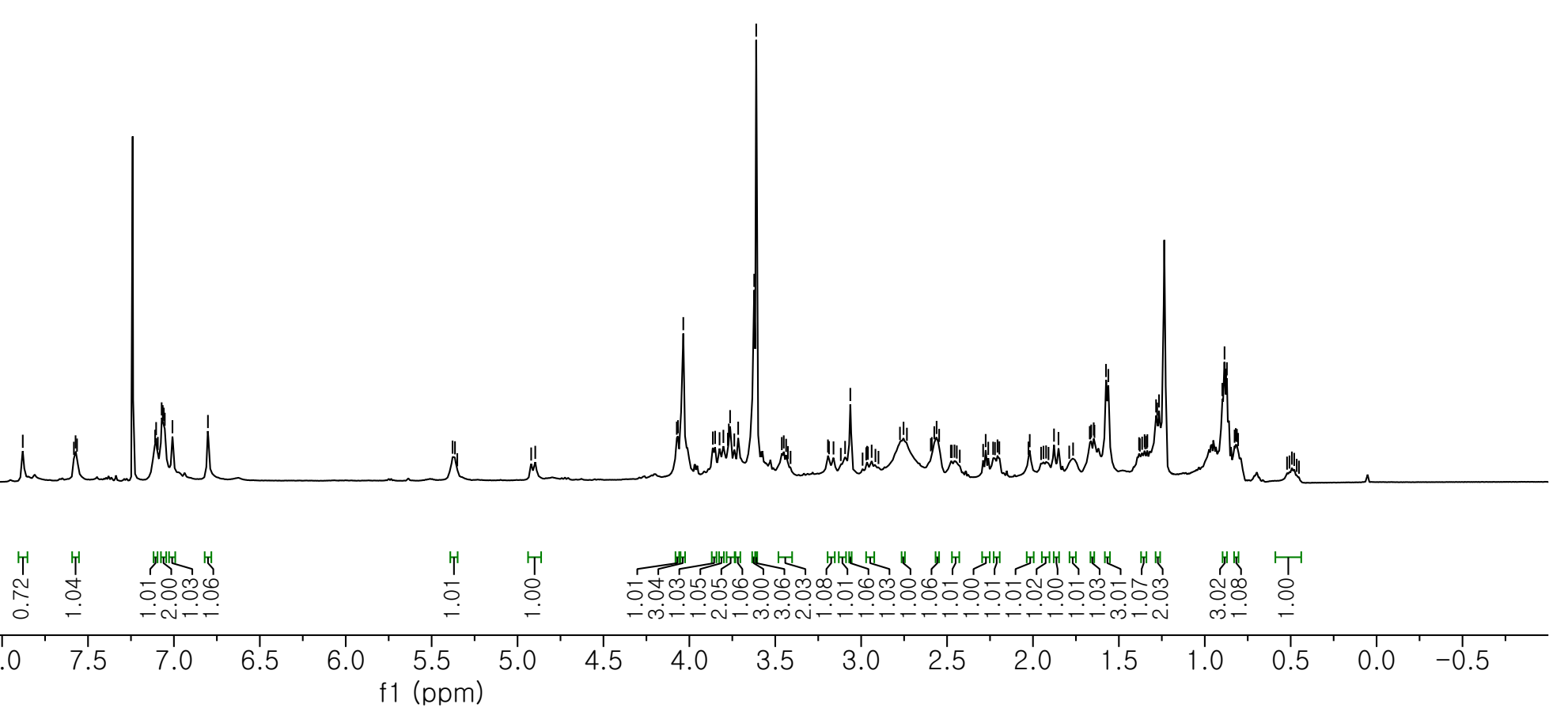




\section{Parameter}

Value

\section{Origin}

2 Instrument

Varian

3 Solvent

4 Temperature

5 Experiment

6 Probe

7 Number of Scans

8 Spectrometer Frequency 150.86

9 Nucleus vnmrs

cdcl3

25.0

10

5mm_One_probe

12714

$13 \mathrm{C}$

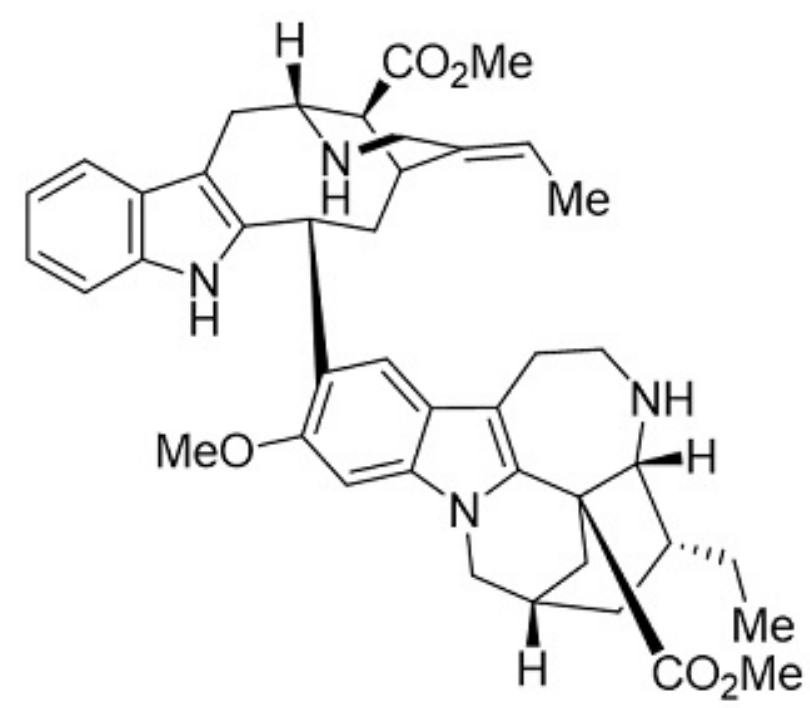

45 


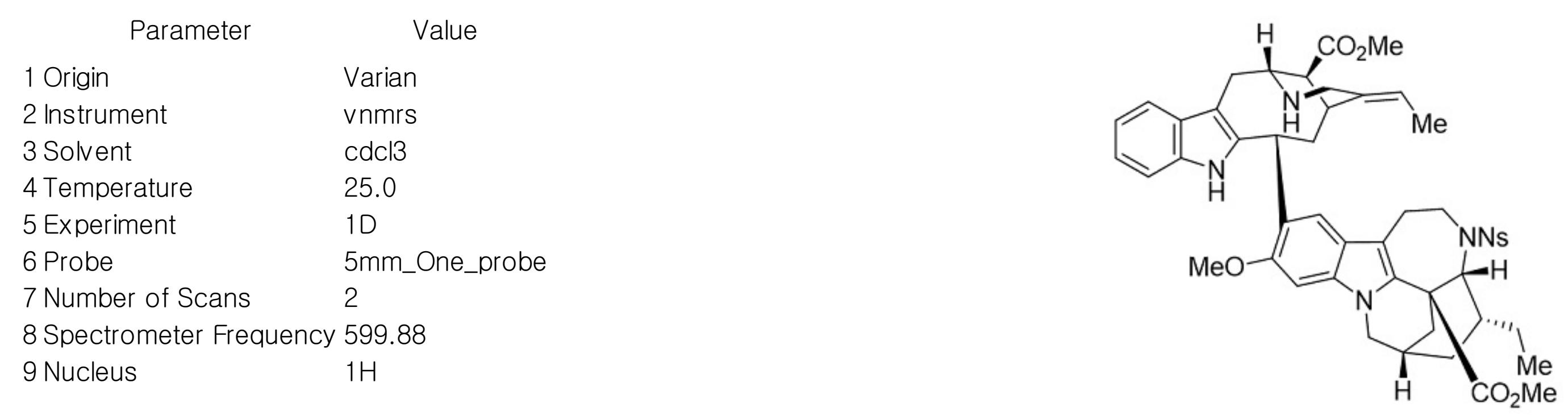

47

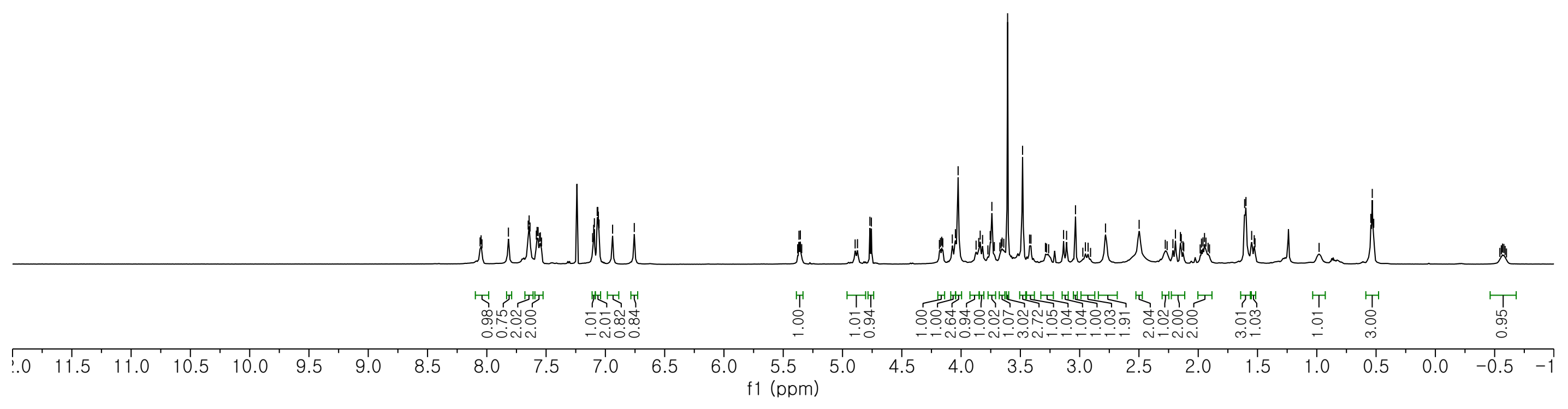


Bio-Patterned Reorganization of Alkaloids Enabled by Ring-Opening Functionalization of Tertiary Amines

Page S179/ S183

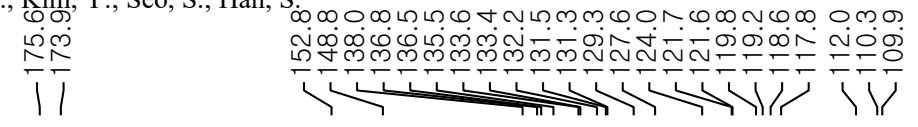

\section{Parameter}

Value

\section{Origin}

2 Instrument

Varian

3 Solvent

4 Temperature

5 Experiment

6 Probe

7 Number of Scans

8 Spectrometer Frequency 150.86

9 Nucleus vnmrs

cdcl3

25.0

10

5mm_One_probe

13202

$13 \mathrm{C}$

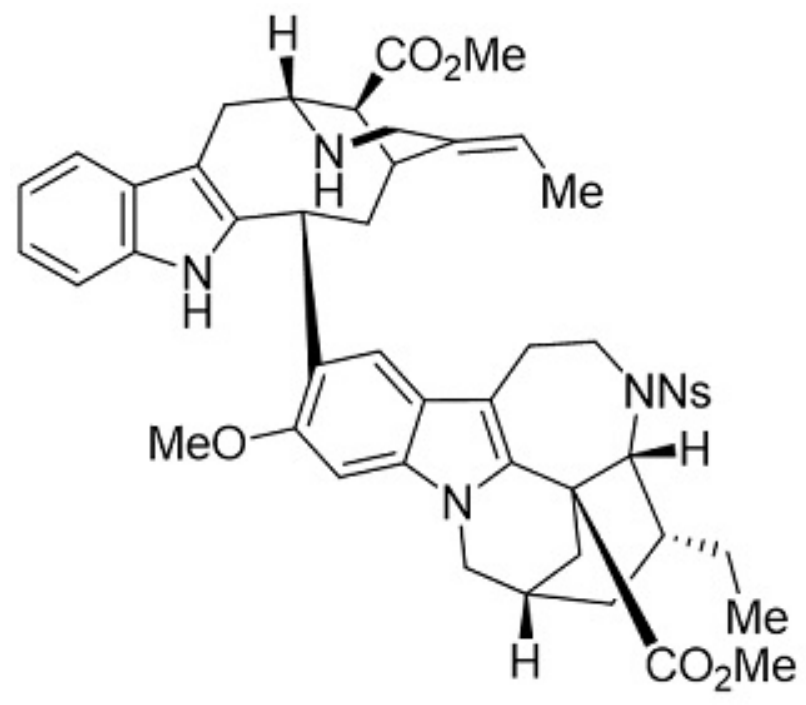

47

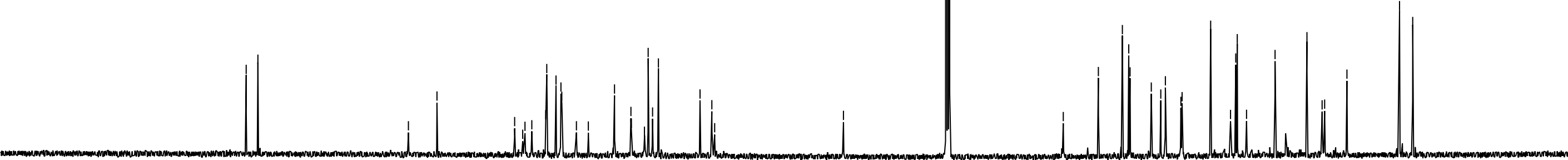




\begin{tabular}{ll}
\multicolumn{1}{c}{ Parameter } & \multicolumn{1}{c}{ Value } \\
1 Origin & Varian \\
2 Instrument & vnmrs \\
3 Solvent & cdcl3 \\
4 Temperature & 25.0 \\
5 Experiment & 10 \\
6 Probe & $5 \mathrm{~mm}$ One_probe \\
7 Number of Scans & 4 \\
8 Spectrometer Frequency & 599.88 \\
9 Nucleus & $1 \mathrm{H}$
\end{tabular}

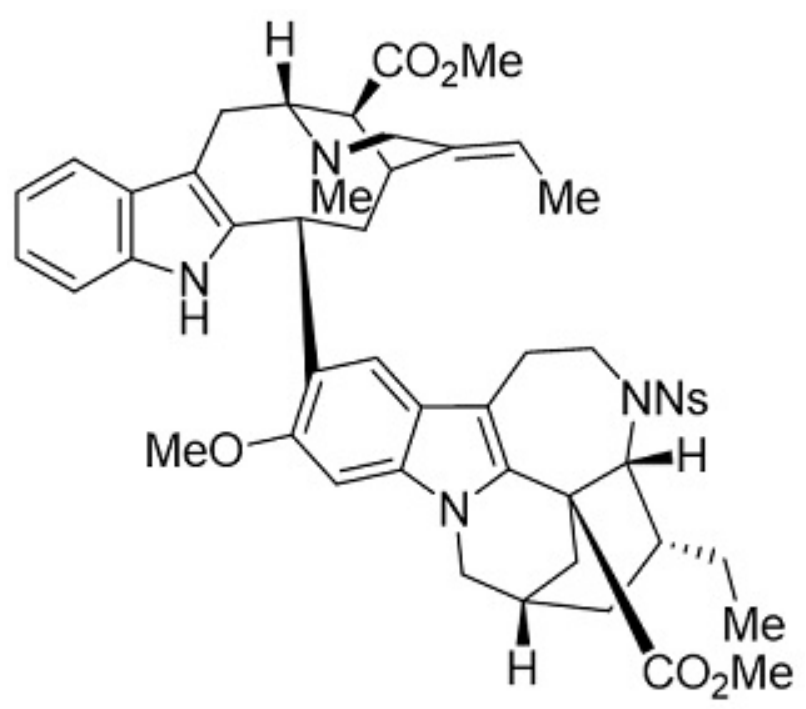

S17

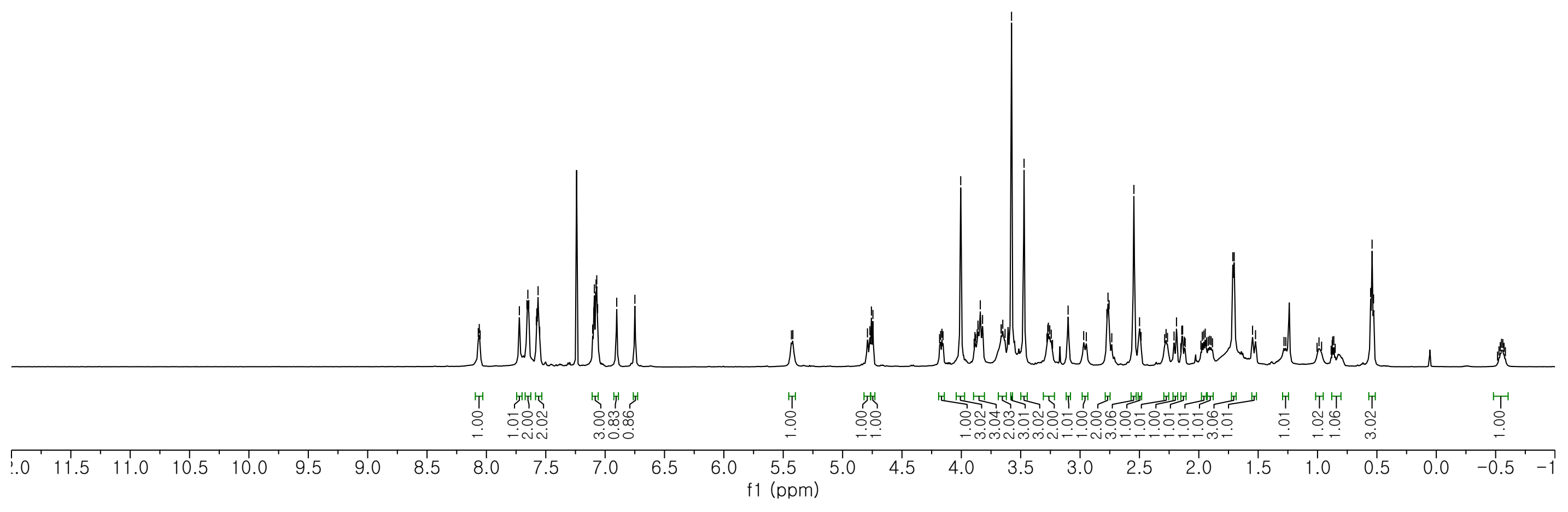


Bio-Patterned Reorganization of Alkaloids Enabled by Ring-Opening Functionalization of Tertiary Amines

|| Page S181 / S183

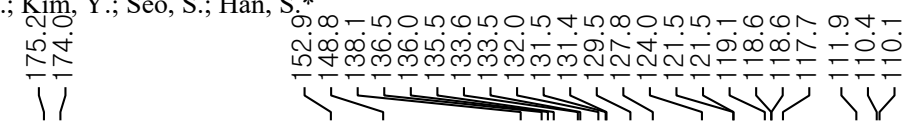

\section{Parameter}

Value

\section{Origin}

2 Instrument

3 Solvent

4 Temperature

5 Experiment

6 Probe

7 Number of Scans

10495

8 Spectrometer Frequency 100.62

9 Nucleus

$13 \mathrm{C}$

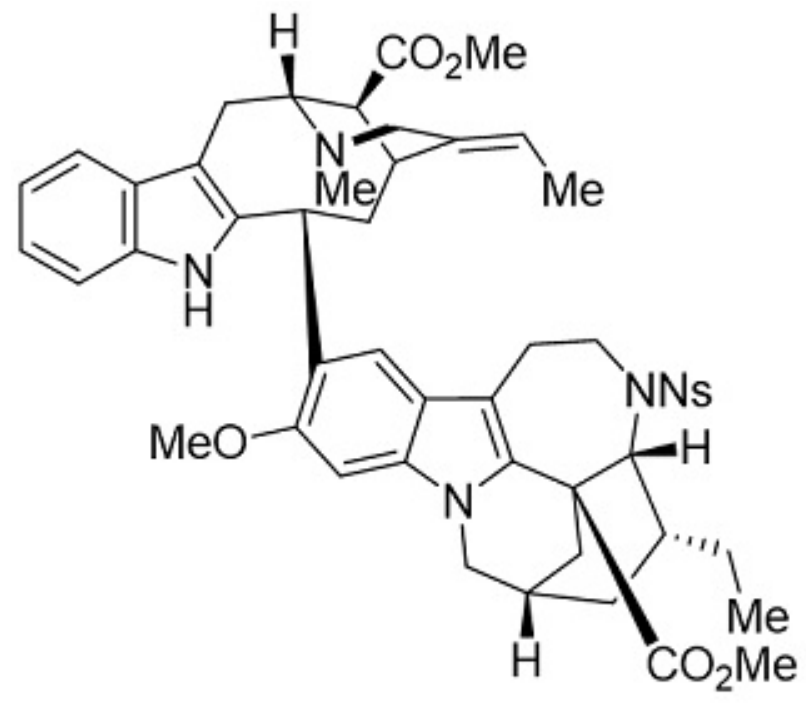

S17

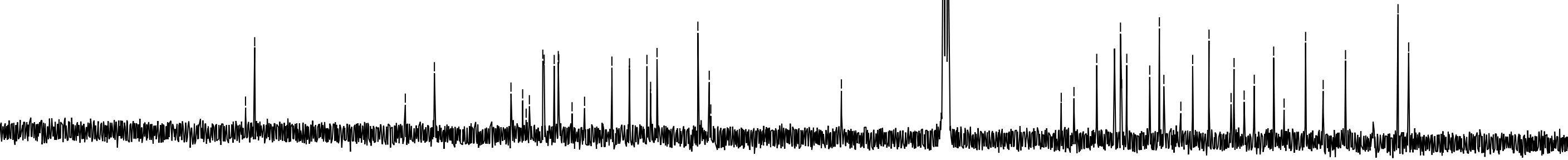




\section{Parameter}

1 Origin

2 Instrument

3 Solvent

4 Temperature

5 Experiment

6 Probe

7 Number of Scans

8 Spectrometer Frequency 599.88

9 Nucleus

$1 \mathrm{H}$

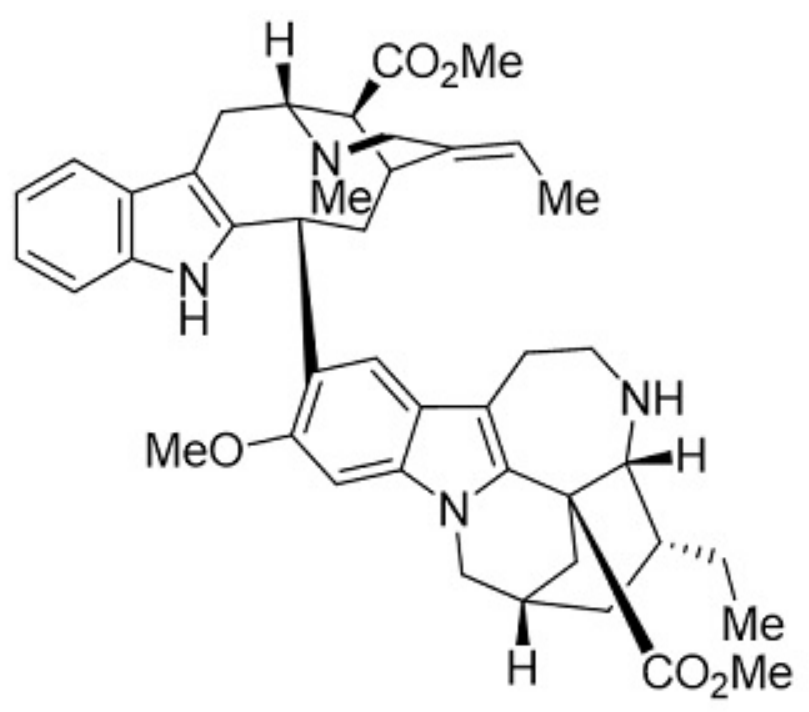

(+)-16-dehydroxymethyl-tabercarpamine A (9)

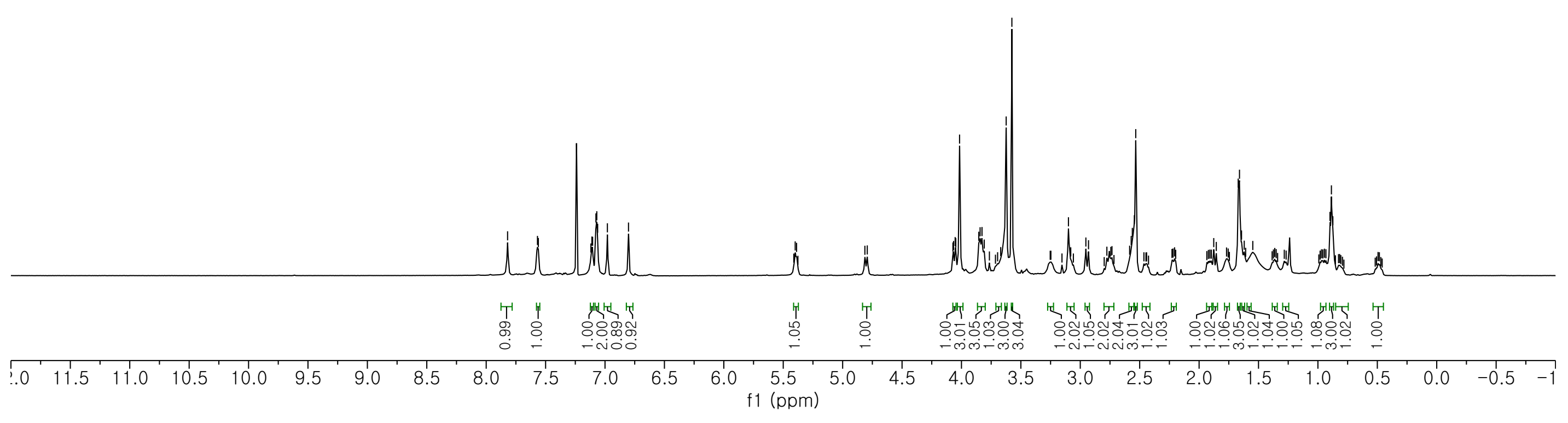


Bio-Patterned Reorganization of Alkaloids Enabled by Ring-Opening Functionalization of Tertiary Amines

Lim, H.; Seong, S.; Kim, Y.; Seo, S.; Han, S.*

\section{Parameter}

Value

1 Origin

2 Instrument

Varian

3 Solvent

4 Temperature

vnmrs

$\mathrm{cdcl} 3$

25.0

5 Experiment

6 Probe

10

5mm_One_probe

12734

8 Spectrometer Frequency 150.86

9 Nucleus

$13 \mathrm{C}$

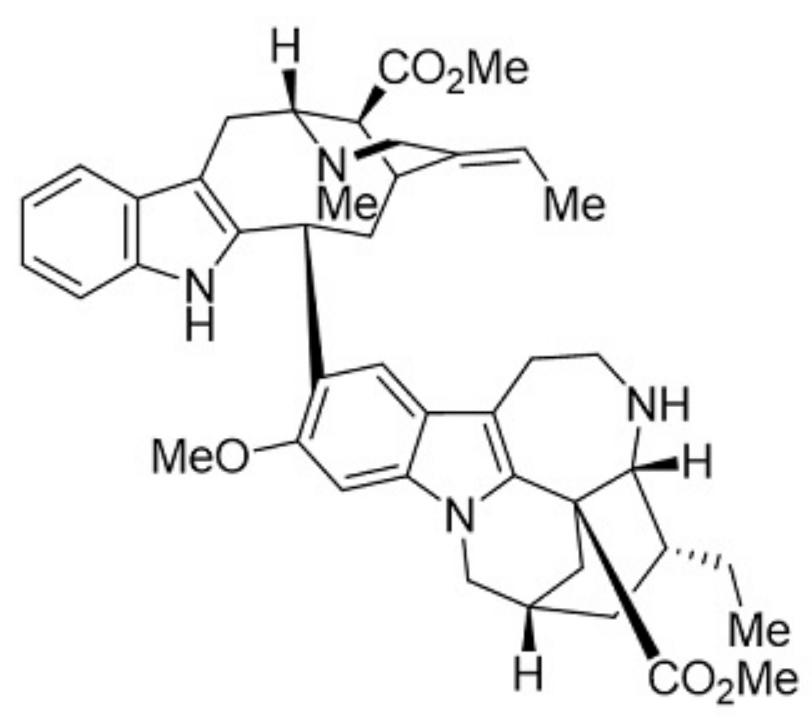

(+)-16-dehydroxymethyl-tabercarpamine A (9) 